\title{
Advanced Computational Thermal Fluid Physics (CTFP) and Its Assessment for Light Water Reactors and Supercritical Reactors
}

Project 2002-016-K

U.S.-RoK International Nuclear Energy Research Initiative Final Technical Report for 11 December 2001 to 31 October 2005
D. M. McEligot
K. G. Condie
G. E. McCreery
H. M. Mcllroy
R. J. Pink
L. E. Hochreiter
J. D. Jackson
R. H. Pletcher
B. L. Smith
P. Vukoslavcevic
J. M. Wallace
J. Y. Yoo
J. S. Lee
S. T. Ro
S. O. Park

October 2005

The INL is a U.S. Department of Energy National Laboratory operated by Battelle Energy Alliance 


\section{Advanced Computational Thermal Fluid Physics (CTFP) and Its Assessment for Light Water Reactors and Supercritical Reactors}

Project 2002-016-K U.S.-RoK International Nuclear Energy Research Initiative Final Technical Report for 11 December 2001 to 31 October 2005

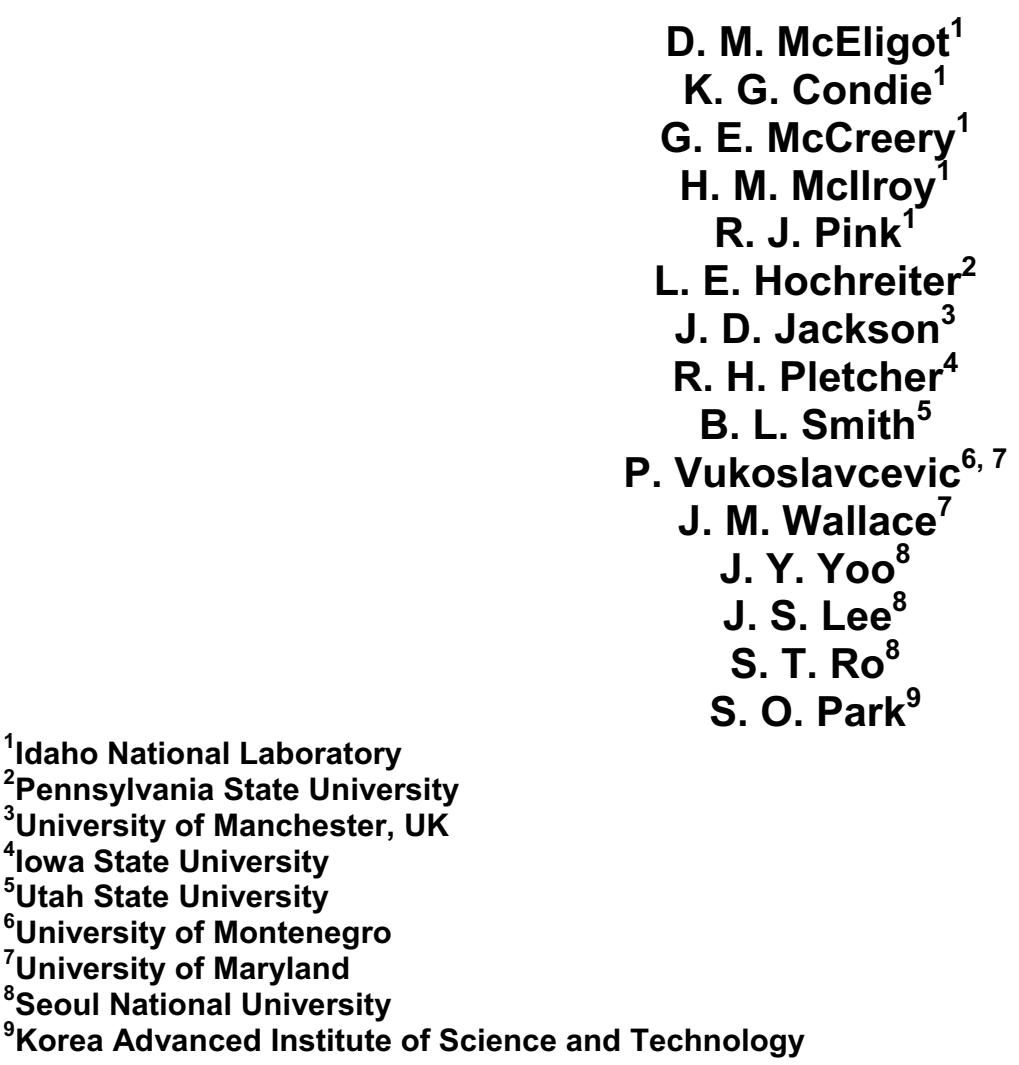

October 2005

Idaho National Laboratory

Idaho Falls, Idaho 83415

Prepared for the

U.S. Department of Energy

Office of Nuclear Energy

Under DOE Idaho Operations Office

Contract DE-AC07-05ID14517 


\section{Table of contents}

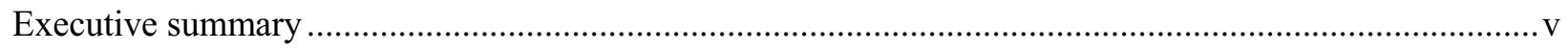

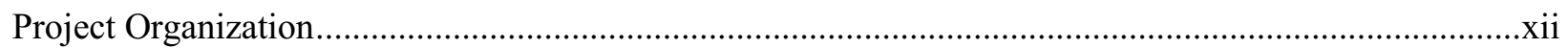

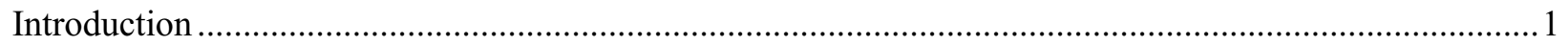

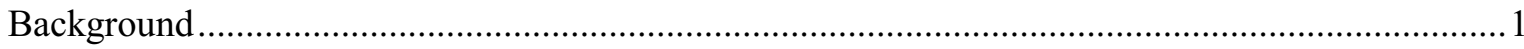

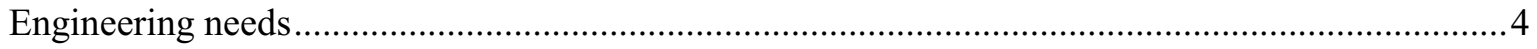

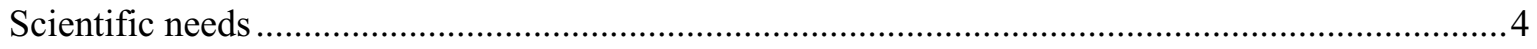

Relations to Generation IV program concepts............................................................................

Task 1. Heat transfer and fluid flow in ALWRs and SCRs..............................................................

Task 2. Direct numerical simulation (DNS) development …...........................................................

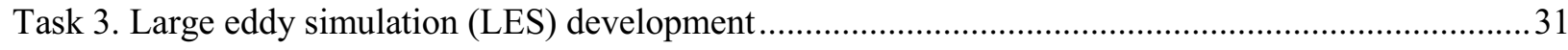

Task 4. Different second-closure (DSM) and Reynolds-averaged Navier-Stokes(RANS) development ..49

Task 5. Complex flow measurements - experiments for assessment …............................................. 75

Task 6. Miniaturized multi-sensor probes for superheated and supercritical flow. ................................ 87

Task 7. Heat transfer experiments - measurements of turbulent quantities in superheated and supercritical

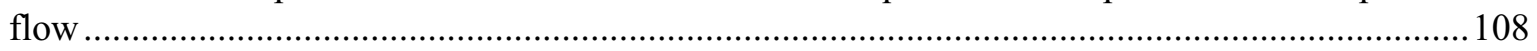

Appendix. Scholarly accomplishments of partners of KNERI project 2002-016-K ............................133

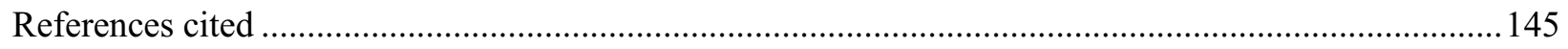




\section{INTERNATIONAL NUCLEAR ENERGY RESEARCH INITIATIVE}

\section{Advanced Computational Thermal Fluid Physics (CTFP) and its Assessment for Light Water Reactors and Supercritical Reactors}

- Principal Investigator (U.S.): D. M. McEligot, Idaho National Laboratory (INL)

- Principal Investigator (Int.): J. Y. Yoo, Seoul National University (SNU)

- Collaborators: Iowa State University; Korea Advanced Institute of Science and Technology (KAIST); Pennsylvania State University; University of Maryland; University of Manchester; Utah State University
- Project Number: 2002-016-K

- Project Start Date: 11 December 2001

- Project End Date: 31 October 2005

- Reporting Period: 11 December 2001 - 31 October 2005

Research objective: The ultimate goal of this Korean / US / laboratory / university collaboration of coupled fundamental computational and experimental studies is the improvement of predictive methods for Generation IV reactor systems (e.g., supercriticalpressure water reactors) and associated Advanced Fuel Cycle Initiative (AFCI) and Nuclear Hydrogen Initiative (NHI) activities. The general objective is to develop the supporting knowledge needed for advanced computational techniques to be used for the technology development of the concepts and their passive safety systems. The resulting specific objectives are to develop and to extend direct numerical simulation (DNS), large eddy simulation (LES) and differential second moment closure (DSM) techniques to treat supercritical property variation and complex geometries, thereby providing capabilities to

- $\quad$ assess predictive capabilities of current codes for supercritical-pressure water reactors (SCWRs), very high temperature gas-cooled reactors (VHTRs), etc.

- $\quad$ provide bases to improve nuclear reactor thermal hydraulics (NuReTH) safety and subchannel codes

- provide computational capabilities where current codes and correlations are inadequate

- give predictions for Generation IV conceptual and preliminary designs for

- $\quad$ full power operation (LES, DSM and Reynolds-averaged Navier-Stokes approaches)

- reduced power operation

- transient safety scenarios, and

- ultimately, handle detailed NuReTH flow problems for final Generation IV designs for improved performance, efficiency, reliability, enhanced safety and reduced costs and waste.

This project provides basic thermal fluid science knowledge to develop increased understanding for the behavior of superheated and supercritical systems at high temperatures, application and improvement of modern computation and modeling methods and incorporation of enhanced safety features. 
Research program: This basic thermal fluids research applies first principles approaches (Direct Numerical Simulation - DNS and Large Eddy Simulation - LES) coupled with experimentation (heat transfer and fluid mechanics measurements). Turbulence is one of the most important unresolved problems in engineering and science, particularly for the complex geometries and fluid property variation occurring in these advanced reactor systems and their passive safety systems. DNS, LES and differential second moment closures (DSM or Reynoldsstress models) are advanced computational concepts in turbulence "modeling" whose development has been extended to treat complex geometries and severe property variation for designs and safety analyses of Generation IV reactor systems such as SCWRs.

Variations of fluid properties along and across heated flows are important in Supercriticalpressure Water Reactors (SCWRs), Very High Temperature gas-cooled Reactors (VHTRs) and Gas-cooled Fast-spectrum Reactors (GFRs), all Generation IV reactor systems concepts. Significant differences and uncertainties have been found between thermal hydraulic correlations for these conditions. Improved computational techniques and supporting measurements are needed to assist the developers of codes for reactor design and systems safety analyses to treat the property variations and their effects reliably for some operating conditions and hypothesized accident scenarios of these reactors. The geometries of the reactor cooling channels of some SCWR concepts are demonstrated in Figure E-1. Most of these geometries are more complex than those that have been used to generate the empirical correlations employed in the thermal hydraulic codes. Advanced computational techniques may be applied but measurements with realistic geometries are needed to assess the reliability and accuracy of their predictions.
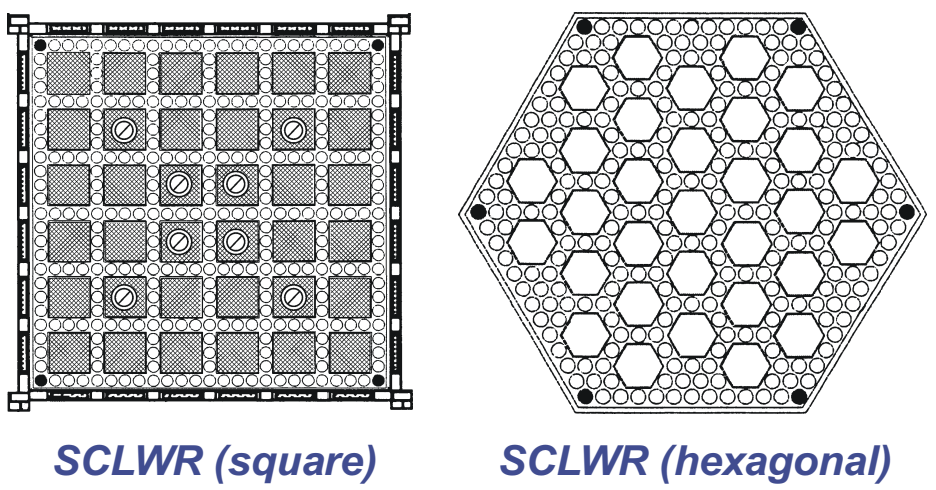

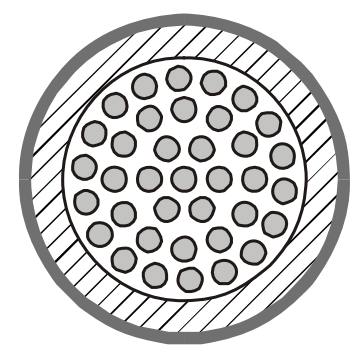

CANDU-X

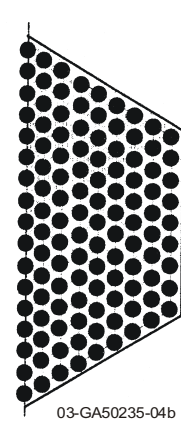

SCFR-H

Fig. E-1. Some proposed designs for fuel assemblies in some Supercritical-pressure Water Reactor concepts.

Prof. R. H. Pletcher (Iowa State) extended LES to generic idealizations of such geometries with property variation; Prof. J. Y. Yoo (SNU) supported these studies with DNS. Prof. S. O. Park (KAIST) developed DSM models and evaluated the suitability of other proposed RANS (Reynolds-averaged Navier-Stokes) models by application of the DNS, LES and experimental results. INL and Prof. B. L. Smith (Utah State) obtained fundamental turbulence and velocity data for an idealization of the complex geometries of these advanced reactor systems. Profs. J. M. Wallace and P. Vukoslavcevic (U. Maryland) developed miniaturized multi-sensor probes to measure turbulence components in supercritical flows in tubes. Profs. J. S. Lee, S. T. Ro and J. Y. Yoo (SNU) developed experiments on heat transfer to supercritical flows. Profs. L. E. Hochreiter (Pennsylvania State) and J. D. Jackson (U. Manchester) provided industrial insight 
and thermal-hydraulic data needs and reviewed the results of the studies for application to realistic designs and their predictive safety and design codes.

DNS employs no turbulence modeling; it solves the unsteady governing equations directly. Consequently, along with measurements, it can serve as a benchmark for assessing the capabilities of LES, DSM and general RANS techniques. It also can be applied for predictions of heat transfer at low flow rates in reduced power operations and transient safety scenarios, such as loss-of-coolant or loss-of-flow accidents, in SCWRs, GFRs and VHTRs. Figure E-2 indicates that for SCWRs it can handle sensitive situations which are difficult to treat properly with correlations or with many turbulence models. Once validated, LES and DSM techniques can be applied for predictions at higher flow rates, such as near normal full-power operating conditions, for these Generation IV reactor concepts. The flow facility developed at SNU provides means of measuring heat transfer to supercritical fluids for assessment of the effects of their property variations and the miniaturized multi-sensor probes from U. Maryland permit measuring the turbulence which is modeled by the codes. The INL experiment models the complex geometry of coolant passages in an SCWR concept to provide benchmark data.
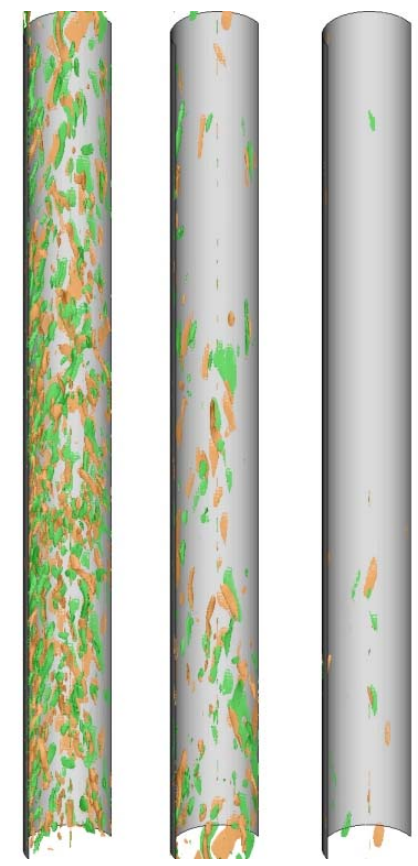

0 10D

10 20D

20 30D

(a) $D=1 \mathrm{~mm}$, upward flow

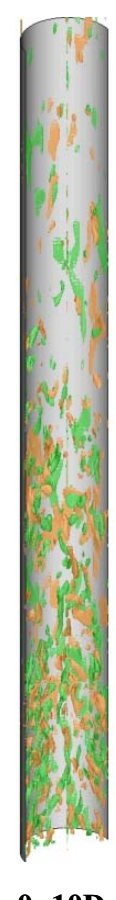

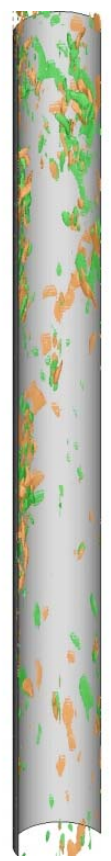

10 20D

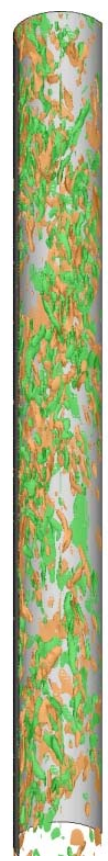

20 30D

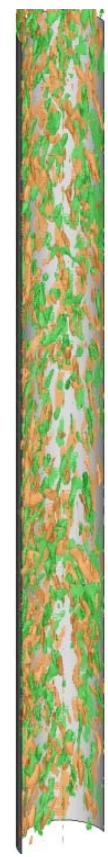

0 10D
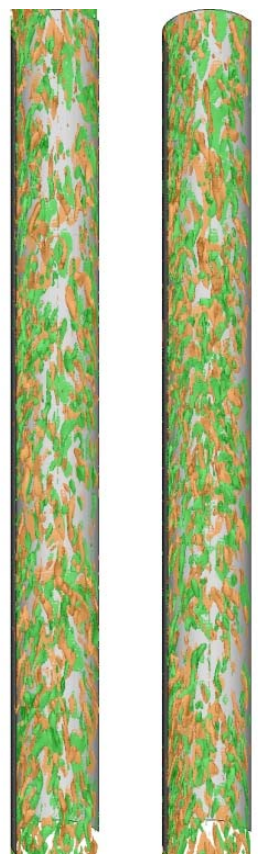

10 20D

20 30D

(c) $D=1 \mathrm{~mm}$, downward flow

Fig. E-2. Direct numerical simulation of heat transfer to supercritical flow demonstrates sensitivity of turbulence (hence heat transfer) to fluid property variation and buoyancy influences.

INL has developed the World's largest Matched-Index-of-Refraction flow system. By using optical techniques, such as particle image velocimetry (PIV), measurements can be obtained in small complex passages without disturbing the flow. The refractive indices of the fluid and the model are matched so that there is no optical distortion. The large size provides 
good spatial and temporal resolution. This facility provides the means to investigate the complex flow features of Generation IV reactor geometries.

Research accomplishments:

- Prof. Yoo extended his DNS code to obtain the first treatment of heat transfer to supercritical fluids by that numerical technique and completed seventeen cases with conditions spanning the pseudocritical temperature; significant effects of buoyancy and property variation on the turbulence were demonstrated. Predictions were compared to measurements from Task 7 . He then extended the code to annular flow in the pseudocritical region with a heated central rod (Figure E-3) and examined the effects of property variation on turbulent heat flux and other turbulent statistics [Bae et al., 2005].
During this collaborative research project

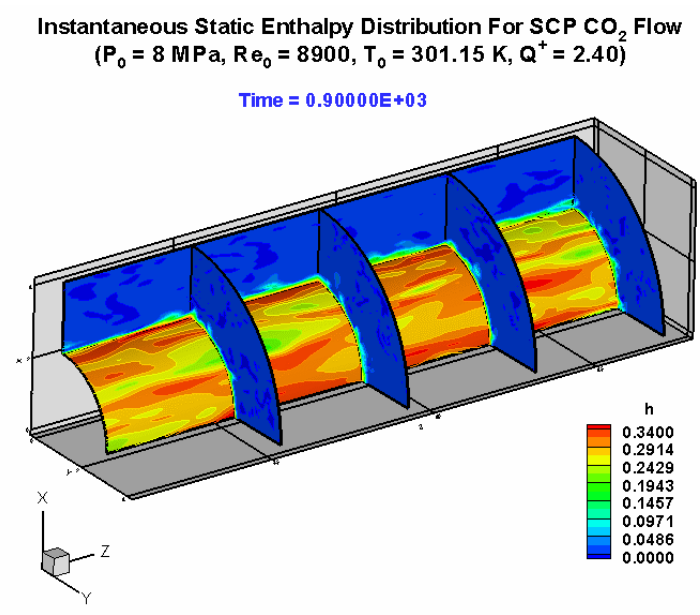

Fig. E-3. Direct numerical simulation of pseudocritical annular flow along a heated rod $\left(h_{p c}=0.0489\right)$. The gas-like region forms a very thin insulating layer near the heated surface, increasing the thermal resistance.

- Prof. Pletcher extended his quasi-developed turbulent LES code for circular tubes to include supercritical fluid properties (Figure E-4) and validated its performance by comparison to DNS (Task 2) and experiments. He then extended it to developing flows and to complex geometries such as annuli, ribbed annuli [McCreery et al., 2003] and an idealization of flow phenomena in coolant channels of an SCWR concept (Task 5).

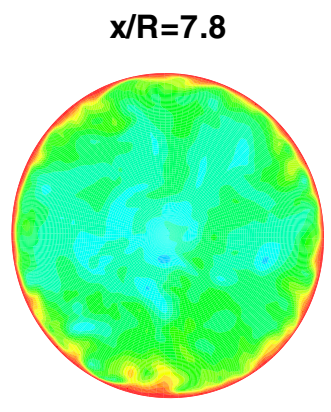

(a)

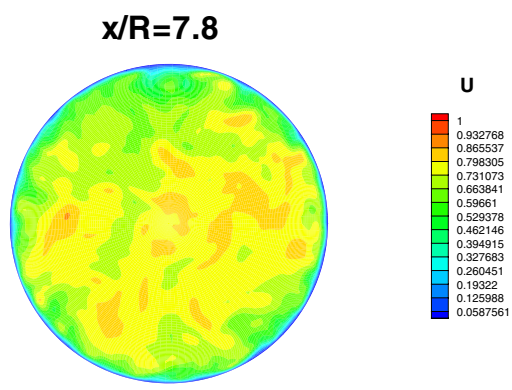

(b)

Fig. E-4. Large eddy simulations of SNU experiment on heat transfer to supercritical $\mathrm{CO}_{2}$ (Task 7 ) at $\operatorname{Re} \approx 28,700$, instantaneous contours: (a) temperature and (b) streamwise velocity.

- Prof. Park applied his DSM code to examine the capabilities of a wide range of turbulence models for heat transfer to superheated gas flows and to supercritical flows -- with and without buoyancy influences -- and compared predictions to DNS (Task 2) and experiments 
(Figure E-5). He found that results depended strongly on the individual models and concluded that one could not be chosen as a best model; some predicted wall temperatures satisfactorily for some cases but not for others. None was universally good.

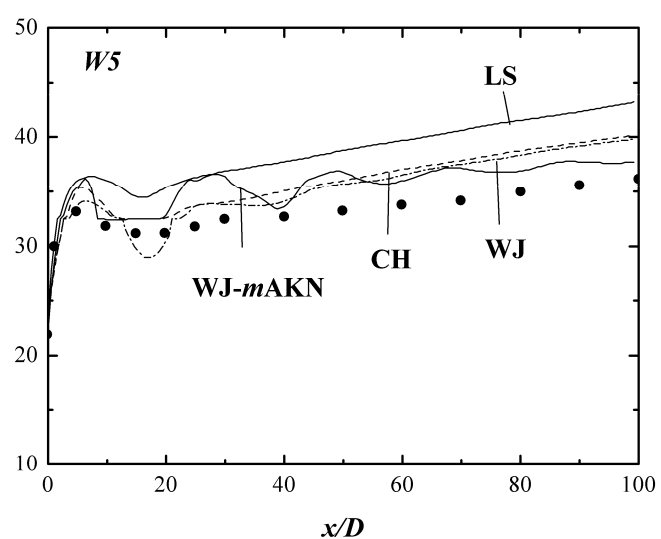

(a)

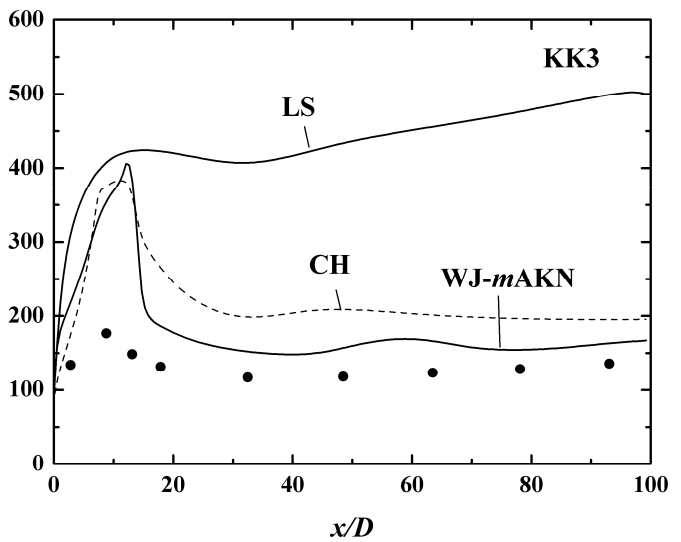

(b)

Fig. E-5. Comparisons of predicted wall temperature profiles for heat transfer to supercritical $\mathrm{CO}_{2}$ flow and data of (a) Weinburg [1972] and (b) Kurganov and Kaptilnyi [1992].

- INL installed a large-scale model for simulating flow in SCWR passages in their MatchedIndex-of-Refraction flow system. With Prof. Smith, they acquired two- and threedimensional PIV data for the streamwise-periodic, three-dimensional region between successive grid spacers (Figure E-6). Results of this benchmark database are archived electronically for assessment of DNS, LES, DSM and RANS codes, as by Task 3 .

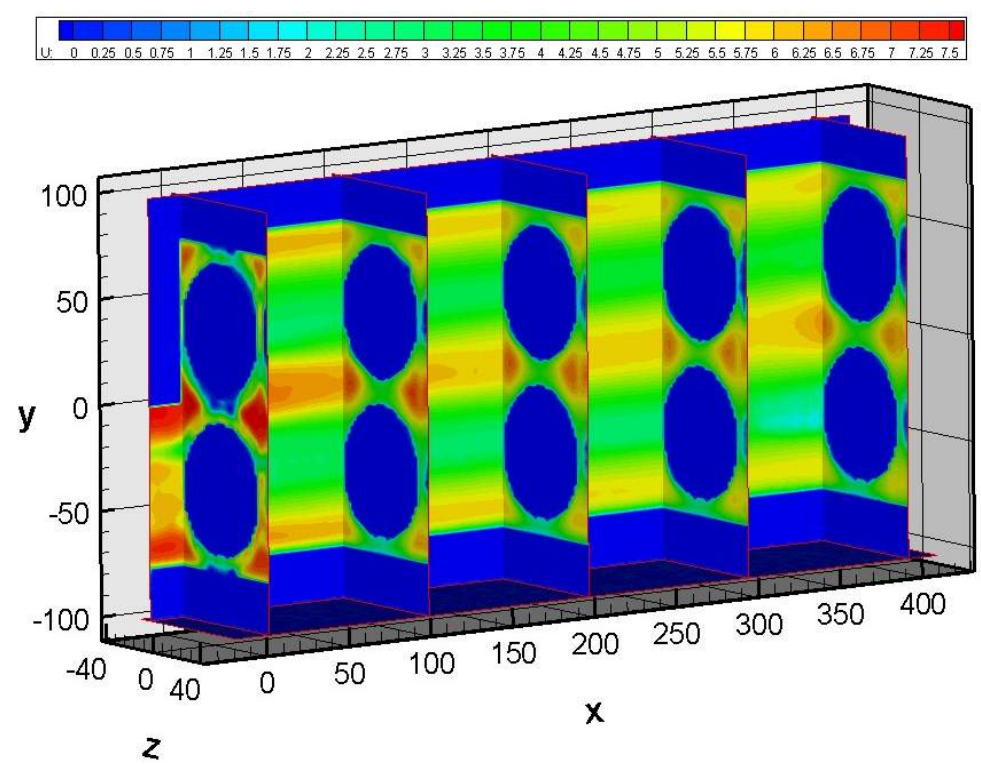

Fig. E-6. PIV (particle image velocimetry) measurements of flow features in a large-scale idealized model of coolant channels in an SCWR fuel assembly [Smith, 2005]. Matching of the refractive indices of the fluid and rod allows undistorted access to the measurement region. 
- Profs. Vukoslavcevic and Wallace developed two-sensor miniaturized hot-wire probes and a calibration facility (Figure E-7) for use in supercritical $\mathrm{CO}_{2}$ heat transfer experiments, calibrated the probes, derived response algorithms and trained SNU students in the use of their probes for measurement of instantaneous temperature and velocities in a supercritical fluid. They designed and constructed a mechanism to traverse the probe inside a high pressure $\mathrm{CO}_{2}$ flow and provided the designs to SNU (Task 7).

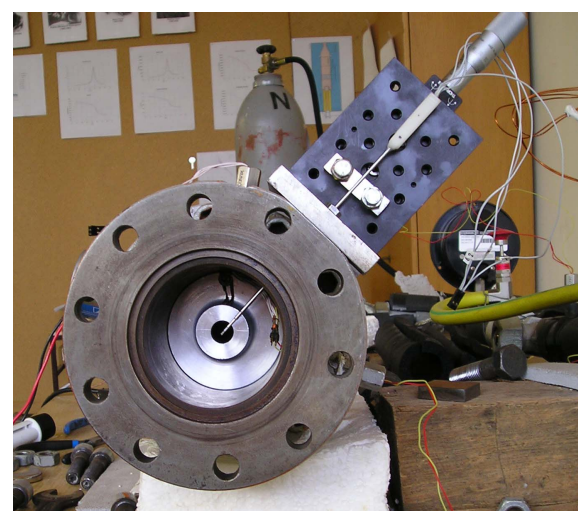

(a)

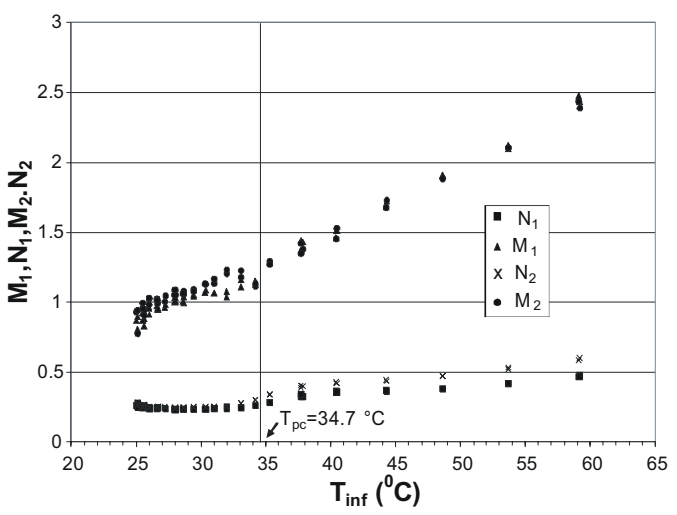

(b)

Fig. E-7. Calibration of two-sensor miniaturized hot-wire probe for determination of velocity, temperature and their turbulent fluctuations in supercritical $\mathrm{CO}_{2}$ flows, (a) photograph of probe mounted in front of nozzle for calibration in heated supercritical flow and (b) correlation of coefficients in convective heat transfer relation for sensor at $\mathrm{p}=8 \mathrm{MPa}$.

- Profs. Lee, Ro and Yoo built an experiment to measure heat transfer, pressure drop and velocity and temperature distributions in supercritical $\mathrm{CO}_{2}$ in tubes. They obtained the first measurements of heat transfer to supercritical flow in small square and triangular tubes (Figure E-8) and measured heat transfer and pressure drop to supercritical $\mathrm{CO}_{2}$ with small and large circular tubes for over 160 sets of conditions overall.

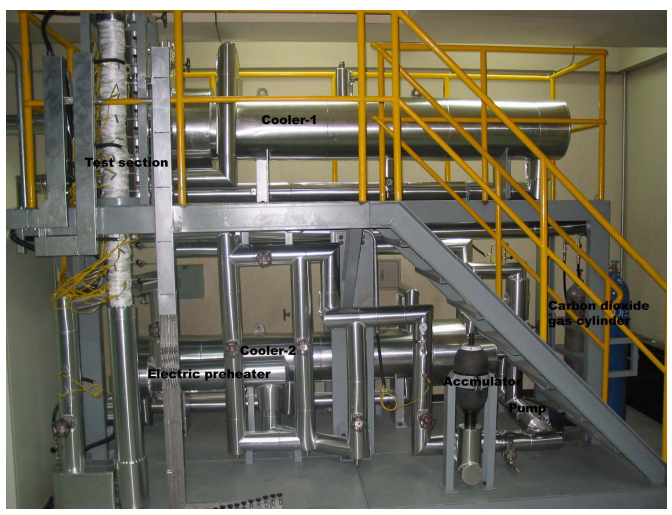

(a)

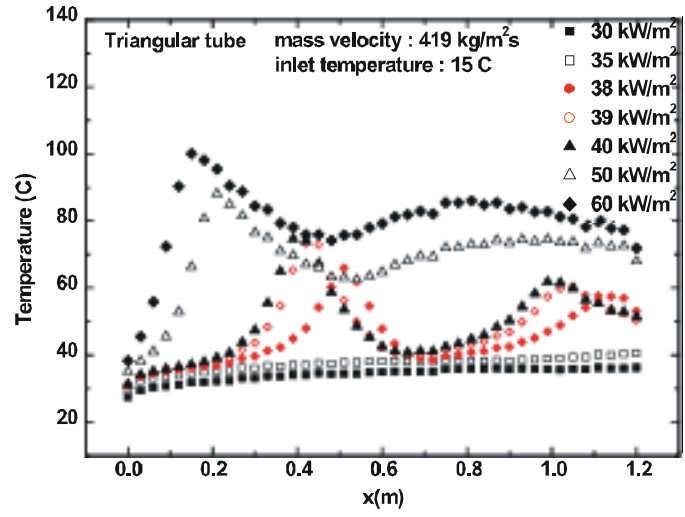

(b)

Fig. E-8. SNU facility for measurements of heat transfer to supercritical carbon dioxide and first measurements for small triangular tubes (hydraulic diameter $=9.7 \mathrm{~mm}$ ). As with circular tubes, "deterioration" is sensitive to the surface heat flux [Kim et al., 2005]. 
Since January 2002, the project partners have had thirty three archival papers published or in press, fifty eight conference presentations and twenty three invited presentations relating to this collaborative Korea/US I-NERI project. They also had over sixty publications on other topics (see Appendix). 


\section{Project Organization}

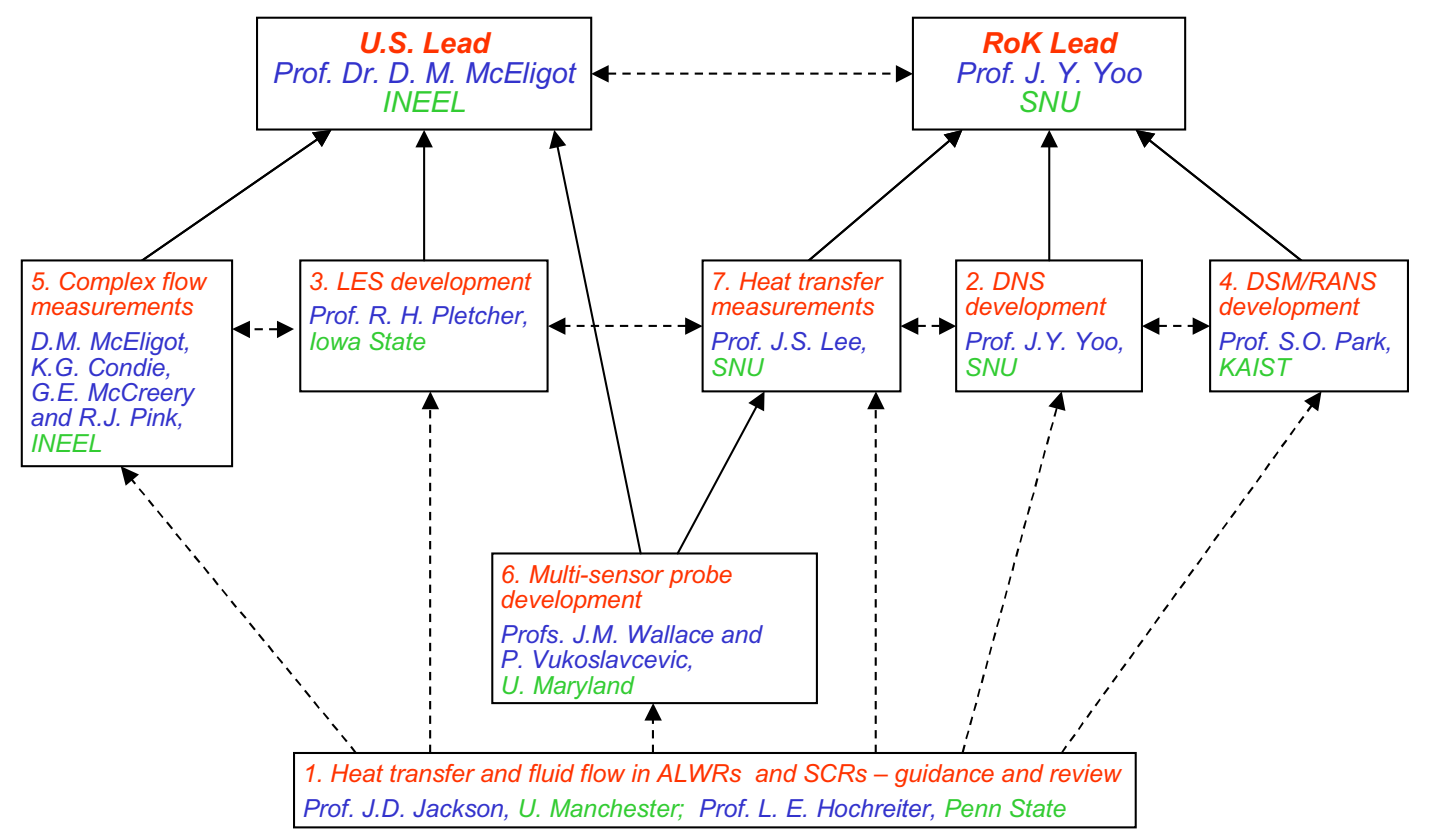

Responsibilities

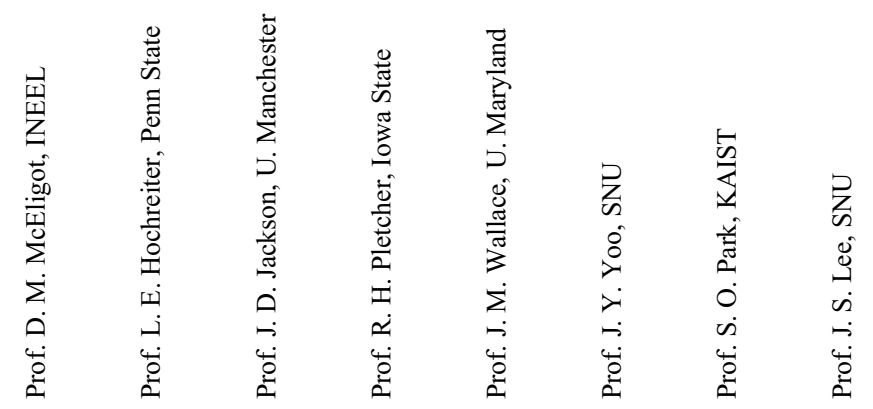

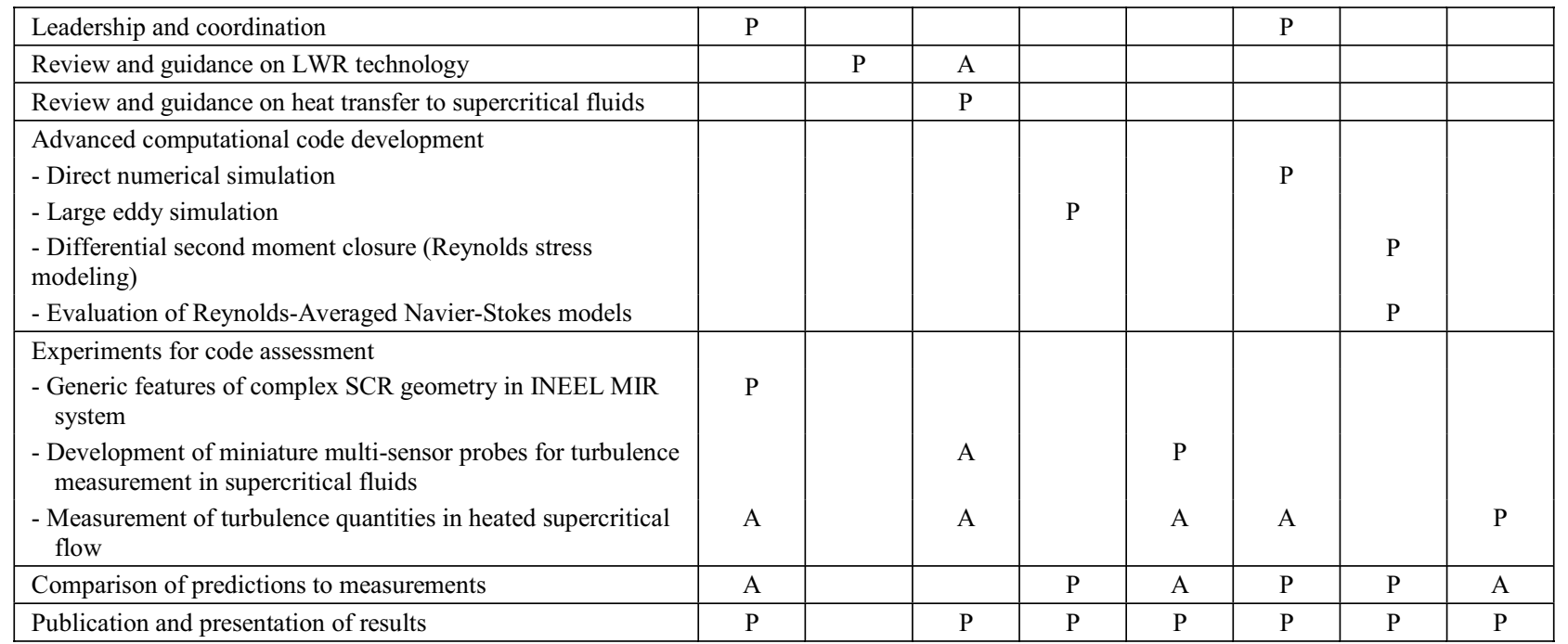

$\mathrm{P}=$ primary responsibility, $\mathrm{A}=$ advise and/or assist 


\section{Introduction}

Background: The ultimate goal of the study is the improvement of predictive methods for safety analyses and design of Generation IV reactor systems such as supercritical water reactors (SCWR) for higher efficiency, improved performance and operation, design simplification, enhanced safety and reduced waste and cost. The objective of this Korean / US / laboratory / university collaboration of coupled fundamental computational and experimental studies is to develop the supporting knowledge needed for improved predictive techniques for use in the technology development of Generation IV reactor concepts and their passive safety systems.

The present study emphasizes SCWR concepts in the Generation IV program. Improved computational thermal fluid physics (CTFP) techniques are needed to account for the complex geometries plus effects of fluid property variation of superheated steam during accident scenarios in Advanced Light Water Reactors (ALWRs) and during all conditions in SCWRs. The resulting specific objectives are to develop and extend direct numerical simulation (DNS, large eddy simulation (LES) and differential second moment closure (DSM) techniques to treat supercritical property variation and complex geometries.

Variations of fluid properties along and across heated flows are important in Supercritical-pressure Water Reactors (SCWRs), Very High Temperature gas-cooled Reactors (VHTRs) and Gas-cooled Fast-spectrum Reactors (GFRs), all Generation IV reactor systems concepts. Significant differences and uncertainties have been found between thermal hydraulic correlations for these conditions. Improved computational techniques and supporting measurements are needed to assist the developers of codes for reactor design and systems safety analyses to treat the property variations and their effects reliably for some operating conditions and hypothesized accident scenarios of these reactors. The geometries of the reactor cooling channels of some SCWR concepts are demonstrated in Figure E-1. Most of these geometries are more complex than those that have been used to generate the empirical correlations employed in the thermal hydraulic codes. Advanced computational techniques may be applied but measurements with realistic geometries are needed to assess the reliability and accuracy of their predictions.

Considerable interest in heat transfer to fluids at pressures just above the thermodynamic critical pressure was stimulated during the 1950s and 1960s by the introduction of fossil-fueled power plants operating at supercritical pressure. Since then little has been done. Recently there has been renewed interest driven by the need to develop innovative nuclear reactors which can compete in terms of cost, safety and reliability with other types of power generation [Oka, 2000]. However, due to the wide variation of fluid properties near the pseudo-critical temperature, the thermal design problem becomes a very complex one which can only be handled satisfactorily in sensitive engineering applications by making judgments based on sound understanding of what is involved [Jackson, 2001].

General effects of strong heating of liquid water are variation of the viscosity and, possibly, significant buoyancy effects depending on the geometry. General effects of strong heating of superheated steam and supercritical water are variation of all transport properties, reduction of density causing acceleration of the flow in the central core, and - in some cases - significant buoyancy forces. Figure I-1a demonstrates the uncertainties involved in applying existing empirical correlations for heat transfer to supercritical flows for the maximum cladding temperature in a current SCWR conceptual design [Davis, 2003]. 


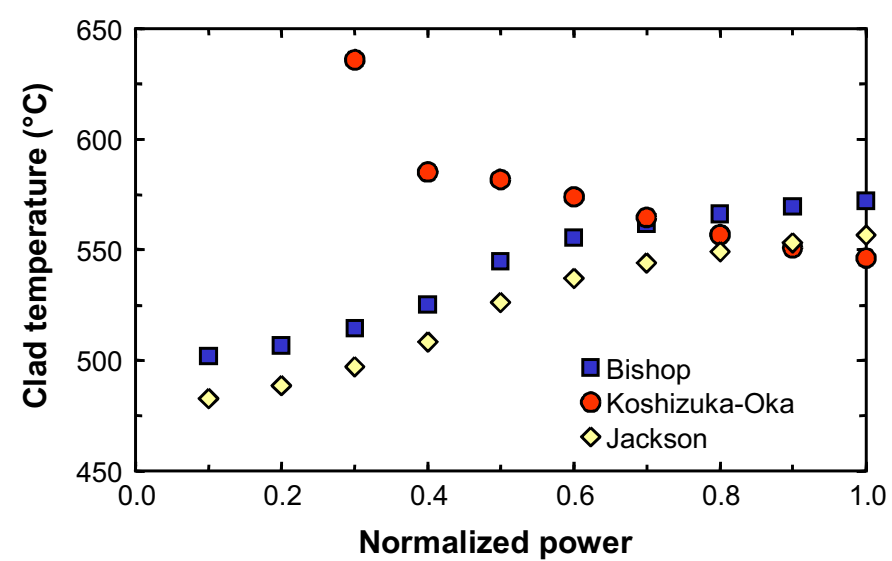

(a)

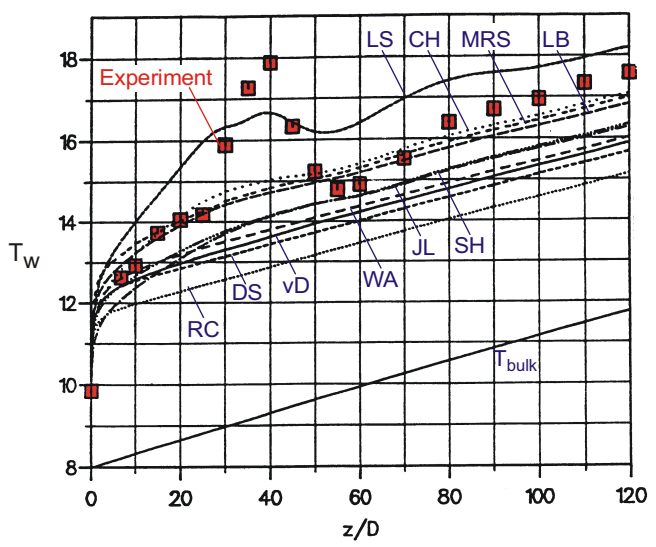

(b)

Fig. I-1. Examples of difficulties in predicting surface temperatures in supercritical flows: (a) use of empirical correlations [Davis, 2003] (b) CFD predictions with popular turbulence models, arbitrary temperature units [Mikielewicz, U. Manchester 1994]

As a single example of consequent application problems --> general-purpose commercial Computational Thermal Fluid Dynamic (CTFD) codes do not necessarily provide accurate or conservative predictions for these conditions, as shown by the comparisons of Figure I-1b. This figure presents predictions from several popular turbulence models which are employed in commercial codes. Figure I-1b demonstrates that the peak wall temperature may occur near the entrance of the heated channel and that all the models tested underpredicted this peak. (While a knowledgeable turbulence modeler might adjust the model so that the predictions agree with the data, the results would not likely be useful beyond the particular situation -- and the normal design engineer does not possess that level of expertise.) Growth of the internal thermal boundary layer leads to readjustment of any previously fully-developed turbulent momentum profile. No truly fully-established conditions are reached because the temperature rises -- leading, in turn, to continuous axial and radial variation of properties such as the fluid viscosity.

Idealized flow geometries are often not found in the reactor cores of light water reactors, either conventional PWRs and BWRs or advanced versions, such as supercritical fluid reactors (SCRs). There are end plates, grid spacers with and without a variety of deflectors, closely-packed fuel rods, stagnant regions and other structures; some examples are shown in Figures E-1 and I-2. Thus, the flow geometry is more complex than the geometries that have been used to generate the empirical correlations used in the thermal hydraulic safety codes. These complex geometries may augment the heat transfer and pressure drop or they may cause stagnation regions with reduced velocities and, thereby, increased thermal resistance leading to "hot spots." Detailed flow field measurements are needed to provide understanding of the interacting phenomena induced by these geometries.

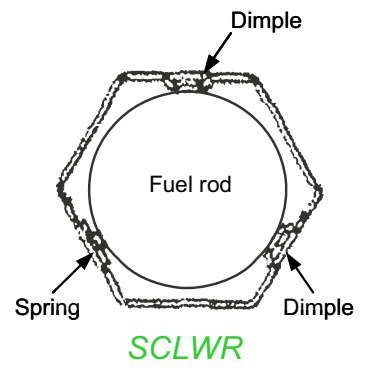

Fig. I-2. Some examples of spacer grids for PWRs, BWRs and SCRs.

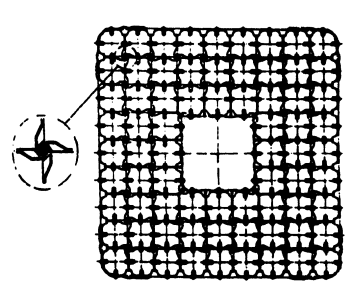

ULTRAFLOW spacer for ATRIUM 9B

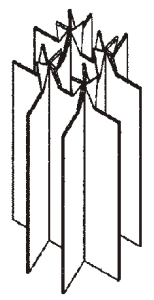

Split-vane grid

Computational thermal fluid mechanics is ubiquitous in reactor thermal hydraulics. Its application is needed for flows in and around complex geometries in ALWRs, SCRs and many other applications. There are several 
vendors who suggest that their commercial general-purpose fluid dynamic codes can solve these problems. However, they do not necessarily provide reliable, accurate or conservative predictions for complex flows. In most applications, these codes depend on turbulence models [Launder and Spalding, 1972] with the most popular being the so-called k- $\varepsilon$ model, a two-equation eddy viscosity model (EVM). However, despite progress in their development, turbulence models are considered the Achilles' heel of modern computational fluid dynamics (CFD) [Hanjalic, 1999]. Predictions of mean velocity profiles which appear reasonable can be misleading, particularly when wall heat transfer and friction are important [McEligot, 1986]. One of the major deficiencies of turbulence models based on the dissipation $(\varepsilon)$ equation is that they are known to predict the onset of separation too late and to underpredict the amount of separation [Menter and Esch, 2001]; many important applications involve separation (e.g., flow behind grid spacers or in the plenum of a reactor). In even a simple flow, such as fully-developed flow of a constant-property fluid in a tube, the predicted heat transfer parameters can be substantially in error depending on the choice of turbulence model [Mikielewicz et al., 2002]. EVM models can serve useful engineering purposes, provided their limitations are known. However, many of their results are misleading and wrong [Hanjalic, 1999]. A thermal design engineer does not necessarily have the expertise to recognize the dangers inherent in application of general-purpose CFD codes.

Some say that turbulence is one of the most important unresolved problems in engineering and science. No workable general theory is known by which the phenomena can be accurately predicted in configurations of practical interest [Pletcher, 1999]. It is well established now that the three-dimensional Navier-Stokes equations along with appropriate forms of the continuity and energy equations govern turbulent flow. Direct numerical simulation (DNS) solves these equations without modeling but limited computer resources inhibit the resolution of flows characteristic of most applications by DNS. This situation is destined to change eventually. As computer hardware and algorithms improve, the frontier will be continuously pushed back, allowing flows of increasing practical interest to be computed by DNS.

Large eddy simulation (LES), in which modeling is required only for the smallest scales, is another approach to simulating turbulence that will benefit increasingly with improvements in computer hardware. In fact, it is believed that the time is right for increased research in this area as more and more practical flow conditions come within reach of current and planned computers. Such an approach requires less computer power than DNS, but is still relatively computer intensive since even when a physical flow appears to be steady and two-dimensional in the mean, LES requires a time-dependent, three-dimensional calculation in order to resolve scales larger than the filter scale. Modeling generally plays a fairly minor role in LES so that quite realistic results are expected. In all known comparisons in complex flows, LES results have been more accurate than those based on the Reynolds-averaged Navier-Stokes (RANS) equations that employ traditional turbulence models. Both DNS and LES approaches permit the calculation of features of the flow that are difficult or impossible to measure. Thus, DNS and LES studies can complement experimental studies of turbulent flow.

Although some commercial vendors advertise LES options, their accuracy and generality have not been demonstrated. The suspicions are that the codes generally simply use their "standard" solvers with a simple subgrid scale model for LES. Apparently some only have the simple Smagorinsky SGS model which probably needs adjustments in order to handle buoyancy, rotation and perhaps other phenomena that a dynamic model can handle. It is not possible for the user to alter the basic commercial code to correct fundamental mistakes.

In the framework of the Reynolds averaging approach, the differential second-moment closures (DSM or Reynolds-stress models) are the logical and natural level at which to model turbulent fluid flow, heat and mass transfer in order to avoid the shortcomings of the EVM models for complex flows. The DSM closures have a sounder physical basis and treat some important turbulence interactions, primarily stress generation, in its exact form. This fact allows better reproduction of the evolution of the turbulent stress field and its anisotropy, streamline curvature, flow and system rotation and flow three-dimensionality [Hanjalic, 1999]. For validation of details of predictions, two-component anemometry can be used to measure more results directly than with k- $\varepsilon$ predictions. The DSM models have been incorporated in some commercial codes but its potential, though long recognized, has not been fully explored or exploited and further developments are needed.

Until recently, application of DNS and LES has been restricted to simple geometries and flows and to low Reynolds numbers due to computer limitations. For practical applications, some further needs in their development 
can be identified by considering an example. Capabilities required of DNS/LES/DSM techniques for typical applications to Generation IV reactor concepts, such as jet flows in plena and cooling of fuel rods and control rods, include

- $\quad$ circular/non-circular geometry

- $\quad$ fluid property variation

- buoyancy

- $\quad$ asymmetric flow obstructions

- $\quad$ variable $\mathrm{q}$ "surf and $\mathrm{T}_{\text {surf }}$ distributions

- $\quad$ semi-confined and confined enclosures

- geometric transitions, inlets/exits

- fully 3-D arbitrary geometry
- $\quad$ cavities

- $\quad$ eccentricity

- $\quad$ arbitrary arrays

- $\quad$ mixing

- $\quad$ swirl flows

- $\quad$ coupled conduction

- crossflows

Satake, Kunugi, Shehata and McEligot [2000, 2002] and Prof. Yoo and colleagues in our present Task 2 have successfully applied DNS to turbulent and laminarizing flow in tubes with variable gas properties and buoyancy. Pletcher and students have developed an LES code for flows in vertical and horizontal channels with fluid property variation and buoyancy [Lee and Pletcher, 2001]; they have extended it to circular tubes and annuli. But, as one sees above, there are many more innovative developments that must be accomplished for DNS/LES applications.

In summary, key features of superheated and supercritical flows in Generation IV reactor concepts for high efficiency are fluid property variation and complex flow passages. These are the topics addressed by the proposed work.

Review of the previous work by the co-investigators and others (Appendix A of the earlier annual report by McEligot et al. [2003]) and of features of superheated and supercritical flow leads to identification of a number of engineering and scientific needs.

\section{Engineering needs include}

- Development of computational techniques for predicting the response of Generation IV reactors during emergency cooling following a loss of forced cooling flow accident.

- Development of computational techniques for mixing of hot jets (streaks) from the core into the plenum during normal reactor operation.

- Development of computational techniques for mixing of hot plumes from the core into the upper plenum during pressurized loss of forced coolant flow.

- Unambiguous "benchmark" data to assess those techniques and to guide further development as necessary.

- Application of computational techniques developed and experimental measurements to the complex geometries of Generation IV reactor concepts.

- Measurements and possible correlation of wall friction for supercritical flows

- Measurements of convective heat transfer and wall friction in non-circular cooling channels (e.g., SCWR fuel assemblies) for supercritical flows

\section{Scientific needs include}

- Determination whether viscous effects or acceleration are responsible for the deterioration of heat transfer parameters and laminarization in heated superheated flows

- Evaluation of criteria for buoyant instabilities and mixed convection in Generation IV reactor geometries

- Measurements of turbulence statistics in strongly heated flows to assist simulation and modeling developments 
- Examination of the effects of flow separation/recirculation and mixing through complex geometries as in SCWR flow channels

Relations to Generation IV program concepts: DNS employs no turbulence modeling; it solves the unsteady governing equations directly. Consequently, along with measurements, it can serve as a benchmark for assessing the capabilities of LES, DSM and general RANS techniques. It also can be applied for predictions of heat transfer at low flow rates in reduced power operations and transient safety scenarios, such as loss-of-coolant or lossof-flow accidents, in SCWRs, GFRs and VHTRs. Figure E-2, 2-12 or 2-21 indicates that for SCWRs it can handle sensitive situations which are difficult to treat properly with correlations or with many turbulence models. Once validated, LES and DSM techniques can be applied for predictions at higher flow rates, such as near normal fullpower operating conditions, for these Generation IV reactor concepts. The flow facility developed at SNU provides means of measuring heat transfer to supercritical fluids for assessment of the effects of their property variations and the miniaturized multi-sensor probes from U. Maryland permit measuring the turbulence which is modeled by the codes. The INL experiment models the complex geometry of coolant passages in an SCWR concept to provide benchmark data. 
Task 1. Heat transfer and fluid flow in ALWRs and SCRs, Prof. L. E. Hochreiter, Pennsylvania State Univ., and Prof. J. D. Jackson, Univ. Manchester

The objectives of this task are to provide insight and guidance to the other partners, to establish which analytical techniques and experimental data are needed for improving the capability of predicting the ALWR and SCR response, to review the proposed computational techniques and their supporting experimental results for their applicability to ALWR and SCR design and to review the results for their applicability to representative geometries and operating conditions under realistic ALWR and SCR operations. This task was primarily accomplished through participation in informal review meetings and small group discussions.

Prof. J. Derek Jackson has considerable first hand experience of heat transfer to fluids at supercritical pressure by virtue of his extensive work on this topic, and also that of his students and colleagues at Manchester University, over a period of about fifteen years during the 1960s and 1970s. In addition to performing experiments, he carried out several detailed reviews and produced a comprehensive Design Report on the subject. He is one of only a very small number of people with such experience who are available to provide authoritative general advice on heat transfer to supercritical pressure water in connection with the recent interest in using it as a coolant in innovative high pressure water-cooled nuclear reactors. He also participated in Task 7 (Heat transfer experiments measurements of turbulent quantities in superheated and supercritical flow) to provide insight, guidance and review.

Lawrence E. Hochreiter is a Professor of Nuclear and Mechanical Engineering at Pennsylvania State University. He worked in nuclear safety for 26 years at Westinghouse. With others he developed the THINC-IV PWR subchannel analysis code for thermal-hydraulic analysis, which was the first subchannel code that included lateral momentum equations to calculate cross flow in rod bundle arrays. He supervised light water reactor safety research for pressurized water reactors. These experiments included large full-length rod bundle blowdown film boiling, level swell and reflood heat transfer tests, the N-RC/W Full Length Emergency Core Heat Transfer (FLECHT) reflooding experiments, the 1/14 and 1/3 scale cold-leg steam/water mixing tests and the Westinghouse Transient DNB tests. He served as Westinghouse safety analysis technical expert for the Three-Mile Island accident and directed an independent Westinghouse analysis of the accident for the President's Commission on TMI. Since joining Pennsylvania State University in 1997, Prof. Hochreiter has continued to work in the safety analysis and development, reactor thermal-hydraulics, reactor safety, two-phase flow and heat transfer areas. He is currently the principal investigator for an NRC-sponsored Rod Bundle Heat Transfer Program, which is designed to provide more fundamental experimental data and model development for the NRC advanced computer codes.

Prof. Hochreiter hosted the initial organizational ("kickoff") meeting for the U. S. collaborators at Penn State during 29-30 July 2002. In addition to presenting a tutorial on PWR design and thermal hydraulic analysis, he reviewed the other presentations on status and plans. Light water reactor (LWR) design and operational limits were discussed as well as the methods used to calculate these limits. Subsequently, he provided a written review of the plans for the proposed supercritical heat transfer experiment at SNU (Task 7).

Prof. Jackson prepared detailed briefing notes on "Heat Transfer to Fluids at Supercritical Pressure" for the benefit of the collaborators on this project and he presented this material to those at the "kickoff" meeting. The presentation began with some background on the proposal to use supercritical pressure water as a coolant in innovative advanced nuclear reactors and an outline of a recent conceptual design. The manner in which fluid properties vary at pressures above the critical value was then described and the consequences of such property variations were outlined in terms of heat transfer. The dimensionless groups involved in convective heat transfer to supercritical pressure fluids were identified by considering the governing equations for flow and energy transfer and the general requirements for similarity were then stated. Attention was next focused on conditions where influences of buoyancy are negligible and a correlation equation for forced convection heat transfer was recommended. A number of special features of heat transfer under conditions of forced convection were then described and illustrated. Finally, buoyancy-influenced heat transfer to supercritical pressure fluids was discussed and some examples were presented of heat transfer behavior under such conditions. Whilst at the "kickoff" meeting he also participated in a review of progress reported by the other collaborators on the project and assisted in the presentation of Prof. Lee's experiment (Task 7).

During 18-23 August 2002, Professor Jackson attended the 12th International Heat Transfer Conference and whilst there he participated in an informal meeting with Profs. Lee, Pletcher, Ro and McEligot at which details of 
the design studies for the supercritical-pressure carbon-dioxide test facility to be constructed at Seoul National University were presented and discussed. He was able to obtain clarification on some details and offer comments and advice. On 17 September Prof. Jackson made a short visit to the AECL Chalk River Laboratories for a separate, but related, project to inspect the supercritical-pressure carbon-dioxide test facility which is being commissioned there and for discussions with AECL concerning their interest and activities concerning heat transfer to supercritical pressure fluids.

At the end of March 2003, Prof. J. Derek Jackson visited our RoK colleagues in Seoul and Taejon, RoK. He participated in the informal workshop on heat transfer to supercritical fluids at SNU organized by Prof. J. Y. Yoo, presenting a talk entitled "Supercritical fluid heat transfer considered in relation to advanced nuclear power plants," visited KAIST and KAERI in Taejon and reviewed the design, construction and plans for the SNU supercritical experiment (Task 7) with Profs. J. S. Lee, J. Y. Yoo and S. T. Ro.

Profs. Hochreiter and Jackson participated actively in the informal annual project review at INEEL during 11-12 June 2003. In addition to presenting background talks, they reviewed and provided constructive criticism for Tasks 2 through 7. Prof. Hochreiter spoke on the general background for light water reactors, grid spacer design and needs of nuclear reactor thermal hydraulics $(\mathrm{NuReTH})$ code developers and users. Prof. Jackson provided a lecture on effects of buoyancy on heat transfer in vertical ducts. On 10 June 2003, Prof. Jackson also presented a seminar entitled "Heat transfer to fluids at supercritical pressure" at INEEL and discussed applications to SCRs with INEEL staff involved in related projects in the Gen IV and NERI programs.

During early October 2003, Prof. Jackson participated actively with Prof. McEligot in attending an informal project review by the Korean partners held at SNU in Seoul. In addition to presenting a background talk on heat transfer to supercritical fluids, he reviewed and provided constructive criticism for Tasks 2 and 7 at SNU

Profs. Hochreiter and Jackson attended the international SCWR Information Exchange meeting in Pittsburgh on 15 June 2004. To guide the partners conducting computational tasks, Prof. Hochreiter initiated a technical note on needs for CFD calculations for a subchannel analysis on coolant flow in supercritical reactors. His presentation at our informal annual review held at Iowa State University during 5-6 August 2004, "CFD needs for sub-channel analysis for fuel assembly design," summarized the background and his guidance.

In addition, Prof. Hochreiter participates in a new international benchmark program on thermal-hydraulics -specifically designed to assess subchannel analysis methods -- that has been initiated between the OECD, NRC and NUPEC of Japan. This program will use subchannel void fraction data and single- and two-phase pressure drop data from the NUPEC rod bundle, as well as the critical power data on different full length BWR rod bundles, for assessment of subchannel analysis methods and computational fluid dynamics methods (CFD). Currently the benchmark program has twenty seven national and international participants who will be using the subchannel analysis methods of their choice. Both traditional subchannel analysis methods and CFD methods will be used. It is expected that this benchmark program will aid in the refinement and development of two-phase flow models for subchannel analyses. The initial meeting was held at the NEUTROS-6 conference in Japan during October 2004.

On 16 May 2005, an International Workshop on the Supercritical Water-cooled Reactor was held at the Institute of Advanced Machinery and Design at SNU. Attendees included SNU faculty and students, Prof. Jackson from the University of Manchester and Prof. Pletcher of Iowa State so the meeting served as a mini-review for our project. Prof. Jackson also presented a talk entitled "Influences on turbulence heat transfer of strong non-uniformity of fluid properties near the critical point." He also attended the Supercritical Water-cooled Reactor Information Exchange Meeting held in conjunction with the ICAPP meeting also in Seoul, Korea 
Task 2. Direct numerical simulation (DNS) development, Prof. J. Y. Yoo, Seoul National Univ. (SNU)

The aim of this task is to develop DNS for use in superheated and supercritical flows with transport property variations and complex non-circular geometries. These results will assist evaluation of sub-grid-scale models used in LES by Prof. Pletcher (Task 3) and of DSM/RANS approach by Prof. Park (Task 4) and will also help in the interpretation of heat transfer experiment results by Prof. Lee (Task 7). The main objectives of the present task are three-fold:

- The first is to develop a numerical method for the prediction of fluid flow and heat transfer with strong fluid property variations;

- The second is to investigate the effect of property variations on the flow and heat transfer in a pipe and to provide valuable information to turbulence modeling study;

- The third is to predict the flow and heat transfer characteristics in tube bank flows with grid spacers, a situation which is closely related to the flows occurring in ALWRs and SCRs.

The present predictions address the effects of fluid property variations and buoyancy forces in vertical flow of a supercritical fluid in the vicinity of its pseudocritical temperature. The present code is directly pertinent to heat transfer in SCWRs operating at reduced power and to LOFA scenarios in an MIT concept for a gas-cooled fast spectrum reactor using supercritical-pressure carbon dioxide [Williams et al., 2003].

During the first and second phases of the present task, Prof. Yoo had developed a DNS code for simulation of pipe flows with fluid property variations. This DNS code of Prof. Yoo solves a set of approximated Navier-Stokes and energy equations known as the low-Mach-number equations for fluid flow and heat transfer with variable physical properties. Although the most general governing equations for variable-property flows are the full compressible Navier-Stokes and energy equations, these classical equations are not well suited for numerical simulation of low speed variable-property flows with heat transfer such as the supercritical flows under consideration. This difficulty is mainly due to numerical stiffness problems associated with a severe time step restriction. In order to circumvent this numerical difficulty, some approximations of the governing equations are necessary. The low-Mach-number equations are obtained based on a standard set of assumptions that acoustic interactions and compressiblity, heating due to viscous dissipation and work terms due to body forces can be safely neglected in low speed, variable-property flows with heat transfer. These assumptions are well justified and accordingly have been frequently used in many previous studies.

To obtain time-accurate solutions using DNS for the low-Mach-number equations, Prof. Yoo has found that a special numerical method is necessary to simulate flows with large density variation such as strongly-heated-gas flows or supercritical-pressure fluid flows. For better treatment of large density variations in the flow, the present DNS code adopted a conservative space-time discretization scheme for variable-density flows [Pierce, 2001]. In this scheme, the low-Mach-number equations are discretized using a conventional second-order finite volume method for spatial derivatives. However, to ensure more accurate and stable discretization of the time-dependent continuity equation, density is staggered both spatially and temporally by one-half grid spacing and by one-half time step, respectively, with respect to each velocity component. This approach makes the numerical scheme for variabledensity flows more conservative so that it proved to be much better in terms of efficiency and accuracy. Further details of this numerical method can be found in the Topical Report of Bae et al. [2005, accepted for publication in Phyics of Fluid].

The code has been successfully validated for various types of flows and heat transfer problems which include (i) laminar flows of gas in a circular tube at high heating rates [Worsoe-Schmidt and Leppert, 1965]; (ii) laminar forced and mixed convection to water near critical region [Lee and Howell, 1996a, b]; (iii) constant-property fullydeveloped turbulent pipe flows [Eggels et al., 1994] and (iv) strongly-heated turbulent internal gas flows [Shehata and McEligot, 1998]. Although the results from these validation studies were already presented in the first and second annular reports of this project in 2002 and 2003, some additional remarks are warranted as follows: First, the predictive capability of the present DNS for variable-property turbulent flows has been well demonstrated by successfully simulating the experiment of Shehata and McEligot [1998] where significant gas property variation occurred due to strong heating rate. In these simulations, a significant impairment of heat transfer coefficient is 
predicted to occur due to a reduction of turbulence leading to relaminarization of the flow. Close investigation of various turbulence statistics indicate that this decay in turbulence is closely related with flow acceleration effects caused by a rapid expansion of the fluid. Second, the usefulness of the low-Mach-number equations for simulating the supercritical-pressure fluid flows near their critical region is well justified by comparing the results for laminar forced and mixed convection with those of the numerical solutions for the full compressible Navier-Stokes and energy equations [Lee and Howell, 1996a, b]. The predicted radial profiles of velocity and temperature distributions are all in excellent agreement with the previous results implying that key physics of supercritical flows are well represented by the low-Mach-number equations.

The present code is based on the cylindrical coordinate system which is best suited for simulations of circular pipe flows and annular channel flows. Figure 2-1 shows a schematic diagram of the flow region and the boundary conditions for the present DNS of vertical pipe flows. As shown in the figure, an inflow generator is used to provide realistic inlet turbulence conditions for a time-dependant turbulent flow simulation of DNS. The inflow generator is a separate computer program running simultaneously with the main code for variable-property flows and it uses a computational domain which is streamwise-periodic to generate a fully-developed flow. As in the inlet conditions, the outlet boundary condition also needs to be time-dependent for turbulent flow simulation. In the present code, this requirement is implemented by using a convective outlet boundary condition. As for thermal boundary condition at the wall, both the iso-thermal and iso-heat flux conditions are considered, but most of the present simulations are made for the iso-heat flux boundary condition. It is also assumed that the heat flux at the wall is mainly determined by conduction only so that thermal radiation effect is not treated in the present simulations.

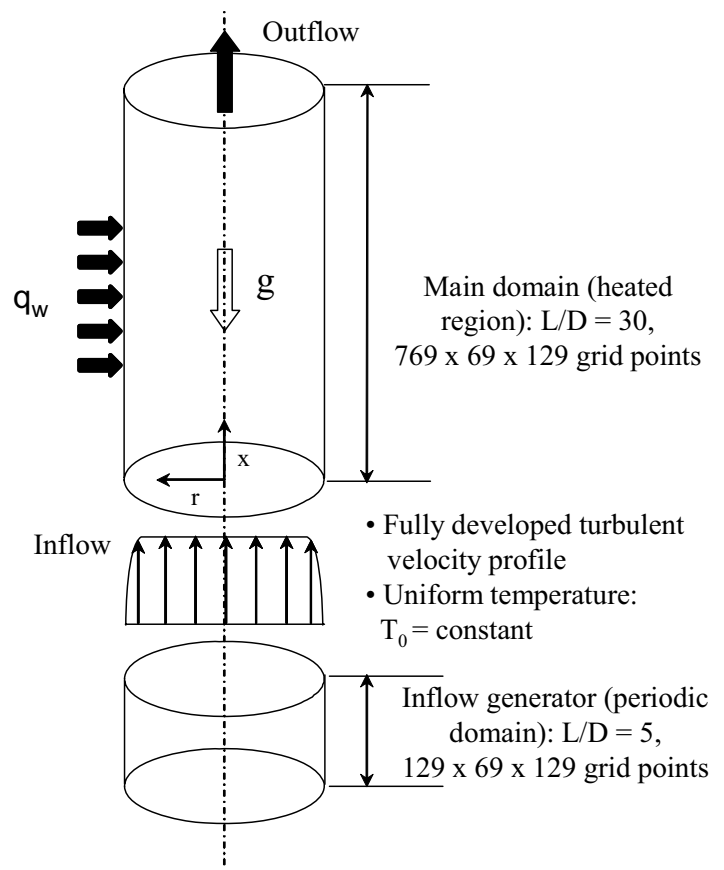

Fig. 2-1: Schematic diagram of the flow region and boundary conditions for upward pipe flows

DNS of vertical pipe flows of supercritical-pressure $\mathrm{CO}_{2} \quad$ Simulations of supercritical-pressure $\mathrm{CO}_{2}$ flowing in vertical tubes have been performed at $P_{0}=8 \mathrm{MPa}$, which corresponds to a reduced pressure ratio of $P_{0} / P_{c}$ $=1.0836$. The critical point of $\mathrm{CO}_{2}$ is $P_{c}=7.3825 \mathrm{MPa}$ and $T_{c}=304.21 \mathrm{~K}$. Figure $2-2$ shows the large property variations of carbon dioxide $\left(\mathrm{CO}_{2}\right)$ at $P_{0}=8 \mathrm{MPa}$. In the present simulations, these fluid property data are obtained using PROPATH [PROPATH Group, 1999] and are incorporated in the form of thermodynamic tables since no closed form of equations is available in the literature. As indicated, all the properties of $\mathrm{CO}_{2}$ at supercritical pressure vary significantly with temperature within a very narrow range across the pseudo-critical temperature $T_{p c}(=307.85$ $\mathrm{K}$ at $P_{0}=8 \mathrm{MPa}$ ) at which $c_{p}$ is the maximum. The pseudo-critical temperature increases with pressure, while the property change becomes less severe as the pressure increases. 


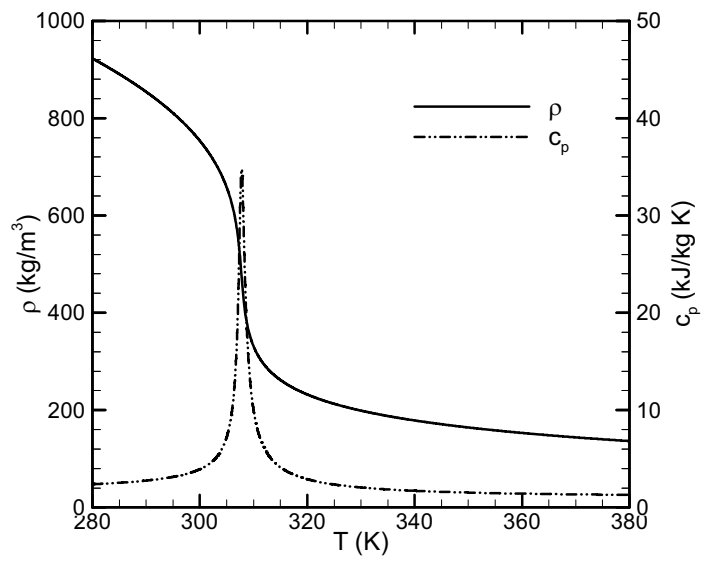

(a)

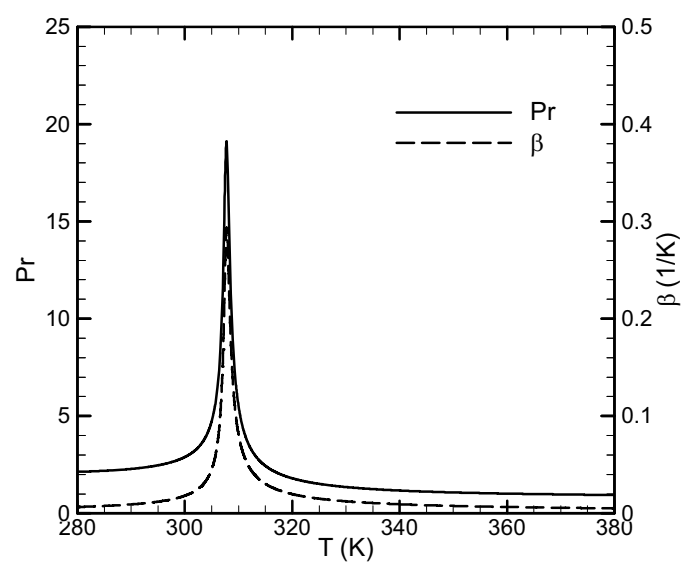

(c)

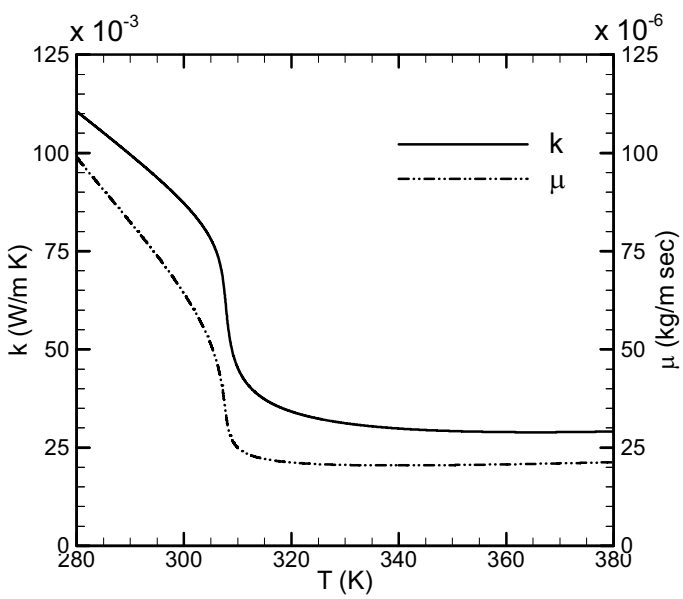

(b)

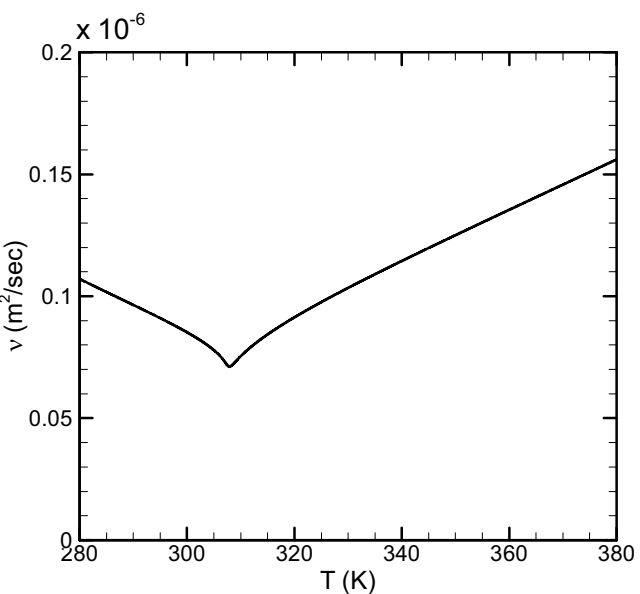

(d)

Fig. 2-2: Physical properties of carbon-dioxide $\left(\mathrm{CO}_{2}\right)$ vs. temperature at $P_{0}=8 \mathrm{MPa}$ : (a) density $\rho$ and specific heat at constant pressure $c_{p}$; (b) thermal conductivity $k$ and dynamic viscosity $\mu$; (c) Prandtl number $P r$ and thermal expansion coefficient $\beta$; (d) kinematic viscosity $v$.

Based on this representation of the property variations, the first series of DNS runs for supercritical-pressure $\mathrm{CO}_{2}$ flowing in vertical tubes have been performed with simulation conditions carefully selected to include the pseudo-critical temperature $T_{\mathrm{pc}}$, where a severe localized deterioration of heat transfer is frequently encountered in experiments [Shiralkar and Griffith, 1970; Ackerman, 1970]. The inlet temperature is chosen as $T_{0}=301.15 \mathrm{~K}$ below $T_{p c}$ and the non-dimensional heat flux at the wall $Q^{+}\left(=q_{w} R / k_{0} T_{0}\right)$ is adjusted to make the wall temperature $T_{\mathrm{w}}$ exceed $T_{\mathrm{pc}}$ at a certain downstream location from the inlet. The inlet Reynolds number is $\operatorname{Re}_{0}\left(=\rho_{0} U_{0} D / \mu_{0}\right)=$ 5400 and the tube length-to-diameter ratio is chosen as $L / D=30$. A wide range of buoyancy parameters is obtained by changing the tube diameter $(D)$ at the same $\operatorname{Re}_{0}, Q^{+}$and $L / D$, so that the buoyancy effect is successfully isolated without changing the other effects of property variations such as the flow acceleration effect, etc. Both upward and downward flows are considered, while forced convection is also simulated to secure the reference data for the buoyancy effect. Table 2-1 shows detailed flow conditions for this series of DNS runs. Case A is forced convection at $Q^{+}=1.2$, and cases $\mathrm{B}$ through $\mathrm{D}$ are mixed convections for upward flow under the same 
heat flux boundary condition but for different tube diameters $D=1,2$ and $3 \mathrm{~mm}$, respectively. Downward flows are also investigated at $Q^{+}=1.2$ by cases $\mathrm{E}$ and $\mathrm{F}$ with $D=1$ and $2 \mathrm{~mm}$, respectively. The effects of increasing heat flux are considered by simulating cases $\mathrm{G}$ and $\mathrm{H}$ at $Q^{+}=2.4$ for upward flows. Buoyancy effects on these flows are estimated by calculating the Grashof number $G r_{0}^{*}\left(=g \beta_{0} q_{w} D^{4} / v_{0}^{2} k_{0}\right)$, buoyancy number $B o$ $\left(=G r_{0}^{*} / \operatorname{Re}_{0}^{3.425} \mathrm{Pr}_{0}^{0.8}\right)$ and $\overline{G r}_{b} / \operatorname{Re}_{b}^{2.7}$, where $\overline{G r}_{b}$ is the local Grashof number based on density difference and $R e_{b}$ denotes the local bulk Reynolds number. These buoyancy parameters are also included in Table 2-1. Table 2-2 shows the flow conditions for the second series of DNS runs in the range of $T_{b}<T_{w}<T_{p c}$ where the property changes are much less significant compared to the first series of DNS runs. For these simulations, the inlet temperature of $T_{0}=295.15 \mathrm{~K}$ is used with a low heat flux of $Q^{+}=0.25$ at the wall.

Table 2-1: Flow conditions for vertical circular pipe flows of supercritical-pressure $\mathrm{CO}_{2}$ at $P_{0}=8 \mathrm{MPa}, T_{0}=$ $301.15 \mathrm{~K}, R e_{0}=5400$ and $P r_{0}=3.08$ (the pseudo-critical temperature is $T_{p c}=307.85 \mathrm{~K}$ at $P_{0}=8 \mathrm{MPa}$ ).

\begin{tabular}{|c|c|c|c|c|c|c|c|c|c|c|c|c|}
\hline Case & Type & Dir. & $\begin{array}{c}D \\
{[\mathrm{~mm}]}\end{array}$ & $\begin{array}{r}T_{0} \\
{[\mathrm{~K}]}\end{array}$ & $\begin{array}{c}G_{0}\left(=\rho_{0} U_{0}\right) \\
{\left[\mathrm{kg} / \mathrm{m}^{2} \mathrm{~s}\right]}\end{array}$ & $P r_{0}$ & $\begin{array}{c}Q^{+} \\
\left(q_{w} R / k_{0} T_{0}\right)\end{array}$ & $\begin{array}{c}q^{+} \\
\times 10^{4} \\
\end{array}$ & $\begin{array}{c}q_{w} \\
{\left[\mathrm{~kW} / \mathrm{m}^{2}\right]}\end{array}$ & $\begin{array}{c}G r_{0}^{*} \\
\times 10^{-8}\end{array}$ & $\begin{array}{r}\text { Bo } \\
\times 10^{5} \\
\end{array}$ & $\begin{array}{c}\left(\overline{G r}_{b} / R e_{b}^{2.7}\right)^{a} \\
\times 10^{4}\end{array}$ \\
\hline $\bar{A}$ & FC & - & 1.0 & 301.15 & 333.24 & 3.08 & 1.20 & 1.44 & 61.74 & 0 & 0 & 0 \\
\hline B & $\mathrm{MC}$ & Up & 1.0 & 301.15 & 333.24 & 3.08 & 1.20 & 1.44 & 61.74 & 0.210 & 0.141 & 0.647 \\
\hline $\mathrm{C}$ & $\mathrm{MC}$ & Up & 2.0 & 301.15 & 166.62 & 3.08 & 1.20 & 1.44 & 30.87 & 1.679 & 1.124 & 4.920 \\
\hline D & $\mathrm{MC}$ & Up & 3.0 & 301.15 & 111.08 & 3.08 & 1.20 & 1.44 & 20.58 & 5.667 & 3.794 & 12.290 \\
\hline E & $\mathrm{MC}$ & Down & 1.0 & 301.15 & 333.24 & 3.08 & 1.20 & 1.44 & 61.74 & 2.099 & 0.141 & 0.538 \\
\hline $\mathrm{F}$ & $\mathrm{MC}$ & Down & 2.0 & 301.15 & 166.62 & 3.08 & 1.20 & 1.44 & 30.87 & 1.679 & 1.124 & 2.973 \\
\hline G & $\mathrm{MC}$ & Up & 1.7 & 301.15 & 196.02 & 3.08 & 2.40 & 2.89 & 72.63 & 2.063 & 1.381 & 3.717 \\
\hline $\mathrm{H}$ & $\mathrm{MC}$ & Up & 2.0 & 301.15 & 166.62 & 3.08 & 2.40 & 2.89 & 61.74 & 3.358 & 2.248 & 5.937 \\
\hline
\end{tabular}

a The values for $\overline{G r}_{b} / \operatorname{Re}_{b}^{2.7}$ shown in the table are the maximum value at each condition.

Table 2-2: Flow conditions for vertical circular pipe flows of supercritical-pressure $\mathrm{CO}_{2}$ at $P_{0}=8 \mathrm{MPa}, T_{0}=$ $295.15 \mathrm{~K}, R e_{0}=5400$ and $P r_{0}=2.48$. Note that the pseudo-critical temperature is $T_{p c}=307.85 \mathrm{~K}$ at $P_{0}=8 \mathrm{MPa}$.

\begin{tabular}{c|c|c|c|c|c|c|c|c|c|c|c|c|c}
\hline \hline Case & Type & Dir. & $D$ & $T_{0}$ & $G_{0}\left(=\rho_{0} U_{0}\right)$ & $P r_{0}$ & $Q^{+}$ & $q^{+}$ & $q_{w}$ & $G r_{0}^{*}$ & $B o$ & $\left(\overline{G r} b / R e_{b}^{2.7}\right)^{a}$ \\
& & & {$[\mathrm{~mm}]$} & {$[\mathrm{K}]$} & {$\left[\mathrm{kg} / \mathrm{m}^{2} \mathrm{~s}\right]$} & & $\left(q_{w} R / k_{0} T_{0}\right)$ & $\times 10^{4}$ & {$\left[\mathrm{~kW} / \mathrm{m}^{2}\right]$} & $\times 10^{-8}$ & $\times 10^{5}$ & $\times 10^{4}$ \\
\hline W & FC & - & 1.0 & 295.15 & 397.00 & 2.48 & 0.25 & 0.373 & 13.80 & 0 & 0 & 0 \\
X & MC & Up & 1.0 & 295.15 & 397.00 & 2.48 & 0.25 & 0.373 & 13.80 & 0.021 & 0.017 & 0.033 \\
Y & MC & Up & 2.0 & 295.15 & 198.50 & 2.48 & 0.25 & 0.373 & 6.90 & 0.170 & 0.136 & 0.343 \\
Z & MC & Up & 5.0 & 295.15 & 79.40 & 2.48 & 0.25 & 0.373 & 2.76 & 2.659 & 2.117 & 4.208 \\
\hline \hline
\end{tabular}

a The values for $\overline{G r}_{b} / R e_{b}^{2.7}$ shown in the table are the maximum value at each condition.

Figure 2-3 shows the radial profiles of the mean velocity and turbulence intensities for fully-developed turbulent pipe flow with constant properties, where the present results are compared with the existing DNS and experimental data of Eggels et al. [1994]. These results are obtained from the inflow generator with 257 x 69 x 129 nodes in the axial, radial and circumferential directions, respectively, and serve as an representative inlet condition for the main simulation of variable-property. As shown in the figure, the mean velocity profile as well as the rootmean-square velocity fluctuations are in excellent agreement with the previous results. 


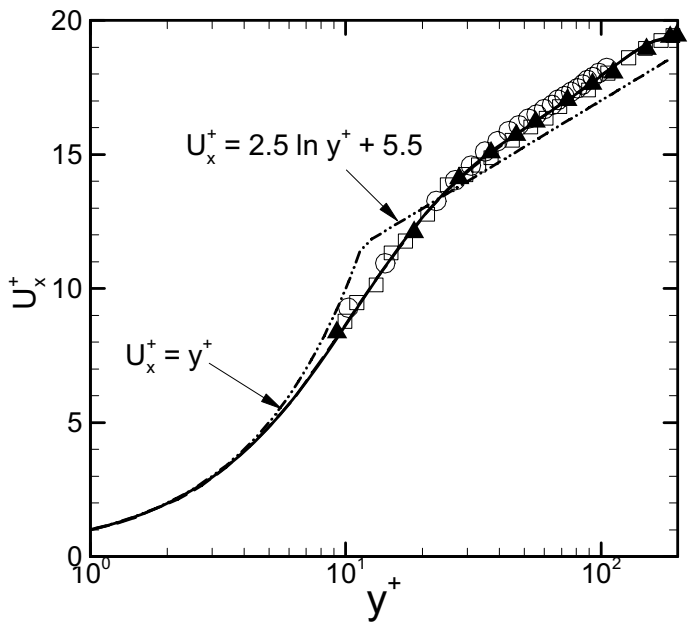

(a)

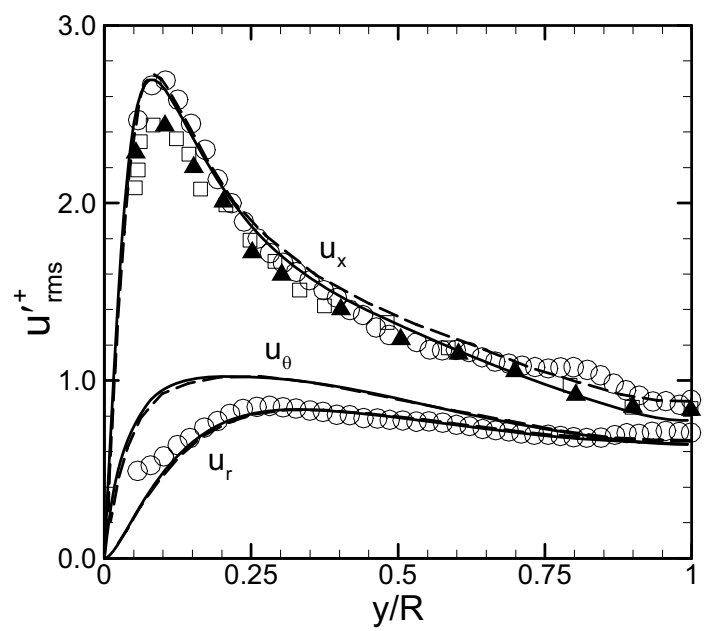

(b)

Fig. 2-3: Simulation results for constant-property, fully-developed turbulent pipe flow at $R e_{0}=5400$ : (a) mean velocity profile in wall coordinates; (b) root-mean-square velocity fluctuations. Eggels et al. [1994]; ๑, HWA; ^, LDA; ○, PIV [Eggels et al., 1994]

The characteristics of turbulent convective heat transfer to supercritical-pressure $\mathrm{CO}_{2}$ are investigated in Figures 2-4 through 2-7. Figure 2-4 shows the streamwise distributions of the non-dimensional bulk enthalpy $\left(H_{b}\right)$ and bulk temperature for cases $\mathrm{A}-\mathrm{H}$, where analytic solution for $H_{b}$ is also included for comparison. The analytic solution for $H_{b}$ can be obtained from the global energy conservation as follows:

$$
H_{b}\left(=\frac{h_{b}-h_{0}}{c_{p 0} T_{0}}\right)=\frac{8 Q^{+}}{\operatorname{Re}_{0} \operatorname{Pr}_{0}} \frac{x}{D} .
$$

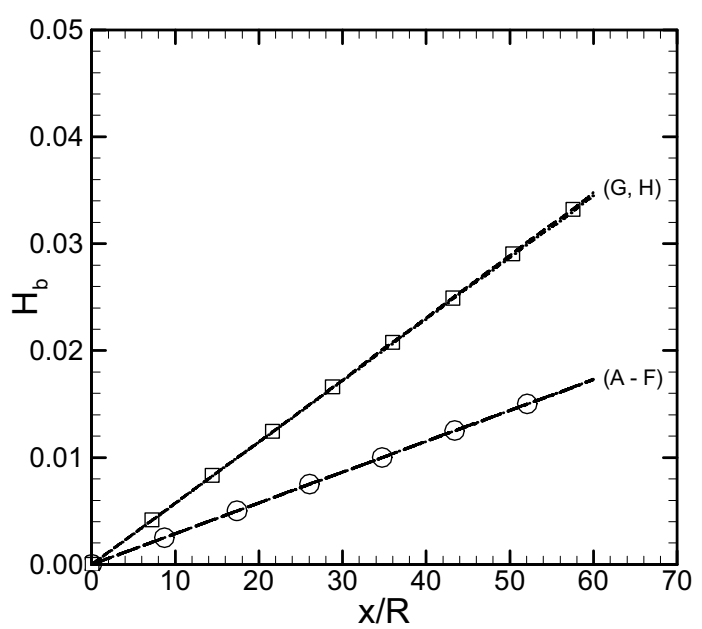

(a)

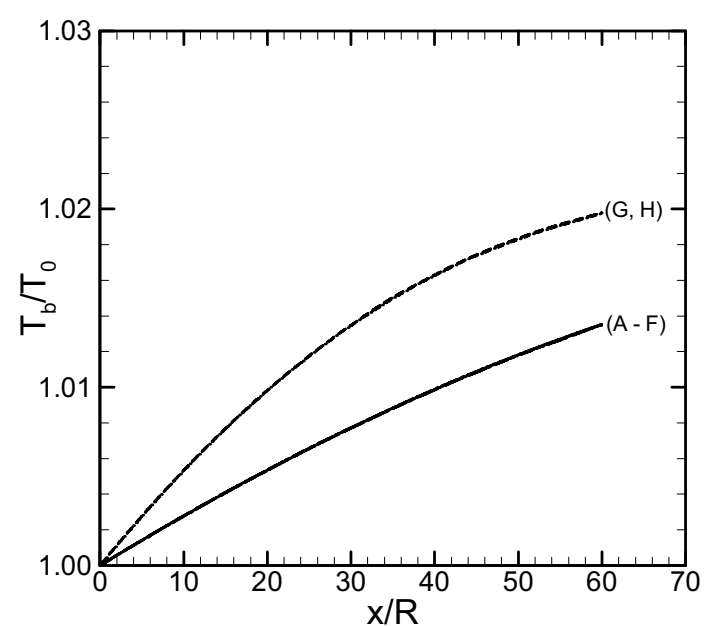

((b)

Fig. 2-4: Nondimensional bulk enthalpy and bulk temperature distributions for cases A - H: (a) $H_{b}=\left(h_{b}-h_{0}\right) /\left(c_{p} T\right)_{0}$; (b) $T_{b} / T_{0}$. 
As expected, $H_{b}$ shows a linear distribution in the streamwise direction while the bulk temperature $T_{\mathrm{b}}$ shows a non-linear behavior because $c_{p}$ is not constant for supercritical-pressure fluids. This non-linear behavior of $T_{b}$ is clearly shown for cases $\mathrm{G}$ and $\mathrm{H}$ at $Q^{+}=2.4$ as $T_{b}$ approaches the pseudo-critical temperature near the outlet $\left(T_{\mathrm{pc}} / T_{0}\right.$ $=1.022$ for these conditions). As clearly shown in the figures, the requirement of energy conservation as well as mass conservation (although not shown here) is accurately satisfied in the present simulations.

Figure 2-5 shows the distributions of non-dimensional wall temperature $T_{w} / T_{0}$ and the local Nusselt number $N u_{b}$ along the streamwise direction for various conditions of buoyancy effect. Cases A through $\mathrm{F}$ are for $Q^{+}=1.2$ and cases $\mathrm{G}$ and $\mathrm{H}$ are for $Q^{+}=2.4$ (see Table 2-1). As shown in Fig. 2-5(a), there are considerable variations in $T_{w} / T_{0}$ depending on the buoyancy parameters. In all these simulations, the condition of $T_{b}<T_{p c}<T_{w}$ is maintained except for a couple of grid points near the inlet, so that significant density change associated with strong buoyancy effect is expected to occur in the cross section. The forced convection result for case A shows a monotonic increase in the wall temperature, as expected since the constant-heat-flux boundary condition is imposed in the present simulation. However, the increase in $T_{w} / T_{0}$ becomes much larger for case B where the buoyancy effect is present but relatively weak. This larger increase in $T_{w} / T_{0}$ is shown to occur because the turbulence is continuously reduced along the streamwise direction for case B. With further increase in the buoyancy effect for cases $\mathrm{C}$ and $\mathrm{D}$, the wall temperature $\left(T_{w} / T_{0}\right)$ begins to decrease along the streamwise direction after reaching peaks in the upstream region. The peaks move further upstream as the buoyancy effect increases while the peak temperature is progressively reduced. In downward flows for cases $\mathrm{E}$ and $\mathrm{F}$, the wall temperature distributions are predicted to be generally lower than those for the upward flows of cases B and C, respectively. Furthermore, wall temperature peaks similar to those found for cases $\mathrm{C}$ and $\mathrm{D}$ are not observed in downward flows and the wall temperature distribution shows much smoother behavior. For cases $\mathrm{G}$ and $\mathrm{H}$ at $Q^{+}=2.4$, variations of $T_{w}$ are much more pronounced than for those cases at lower heat flux condition. Moreover, the wall temperature increases again after reaching a local minimum near the outlet. Indeed, these observations of the predicted peculiar wall temperature distributions for supercritical-pressure $\mathrm{CO}_{2}$ are consistent with the ones based on the previous experimental data which are well summarized in the review by Hall [1971].

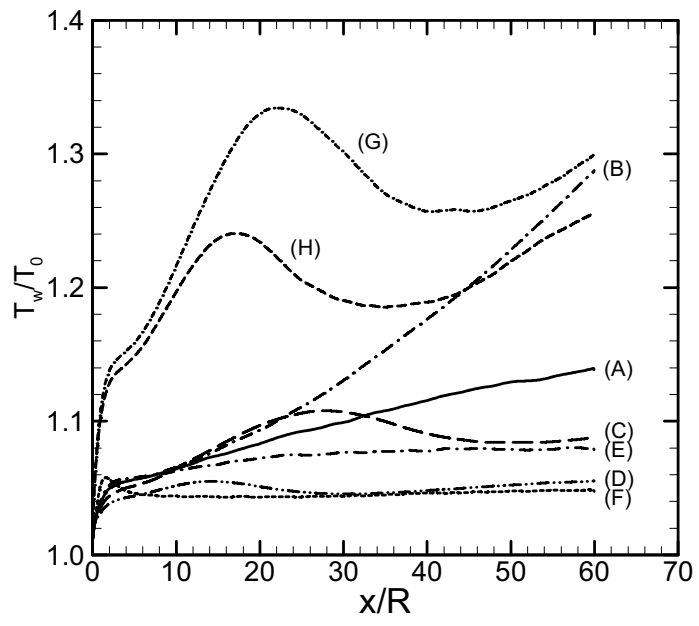

(a)

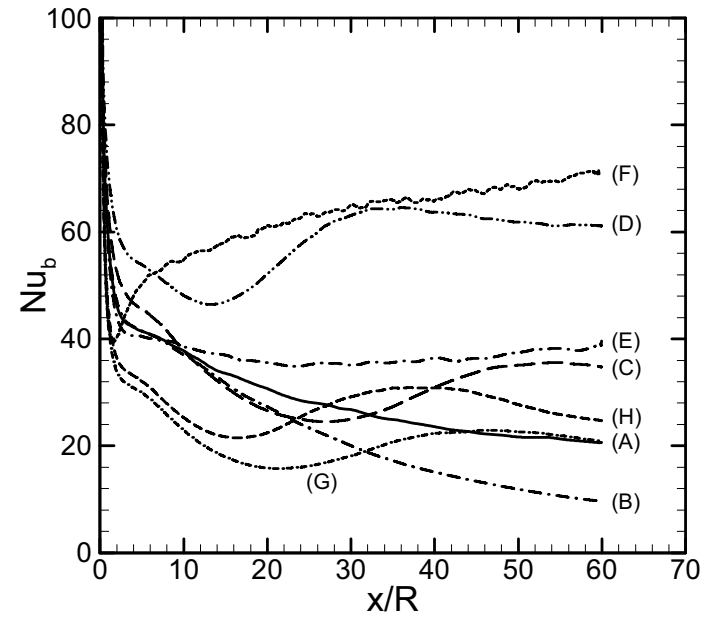

(b)

Fig. 2-5: Nondimensional wall temperature and Nusselt number distributions for cases $\mathrm{A}-\mathrm{H}$ : (a) $T_{w} / T_{0}$; (b) $N u_{b}$.

In Fig. 2-5(b), the streamwise distributions of the local Nusselt number $N u_{b}\left(=h D / k_{b}\right)$ are shown for cases $\mathrm{A}-\mathrm{H}$, where $h$ is the local convective heat transfer coefficient given by $h=q_{w} /\left(T_{w}-T_{b}\right)$ and $k_{b}$ is thermal conductivity evaluated at the local bulk temperature. Since $N u_{b}$ is mainly determined by the difference between the wall and bulk temperatures of which the behaviors have been already examined, no further elaboration may be necessary. However, it should be very interesting to compare the predicted Nusselt number with existing experimental data although the Reynolds number ranges are quite different. Figures 2-6 and 2-7 show comparisons 
of the Nusselt number ratios between the present DNS and the experiment of Fewster [see Jackson and Hall, 1979b] for mixed convection of supercritical-pressure $\mathrm{CO}_{2}$ in circular pipes. In these figures, the ratios of the local Nusselt number for mixed convection $\left(N u_{b}\right)$ to that of forced convection $\left(N u_{f}\right)$ are plotted in terms of buoyancy parameter $\overline{G r}_{b} / R e_{b}^{2.7}$, where $N u_{f}$ is obtained from the modified Krasnoschekov and Protopopov correlation [see Jackson and Hall, 1979a] and $\overline{G r}_{b}$ denotes the local Grashof number based on density difference [Jackson and Hall, 1979a]. Figures 2-6(a) and (b) show the results for upward and downward flows, respectively, for those conditions listed in Table 2-1, and Figure 2-7 is for the conditions in Table 2-2. Data for $x / R \leq 10$ are not included in the figures because the thermal entrance effect is concentrated there, and the solid symbols represent the present results. As clearly shown in these figures, the normalized Nusselt number ratios for upward and downward flows in the present DNS are in excellent agreement with the experimental data when plotted in terms of $\overline{G r}_{b} / \operatorname{Re}_{b}^{2.7}$, although the present results are obtained at much lower Reynolds numbers than the experimental ones.

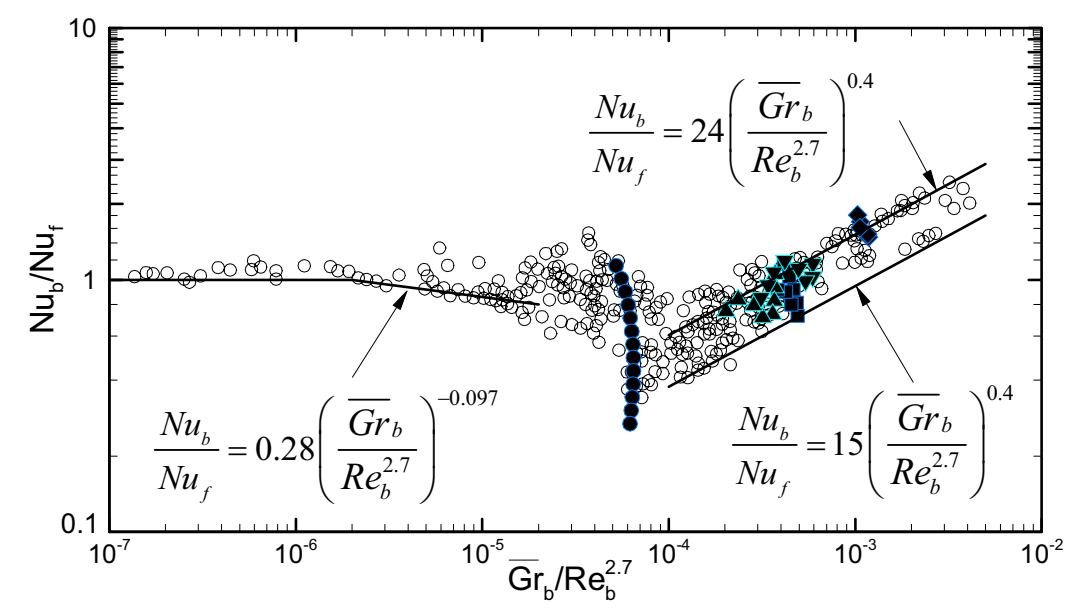

(a)

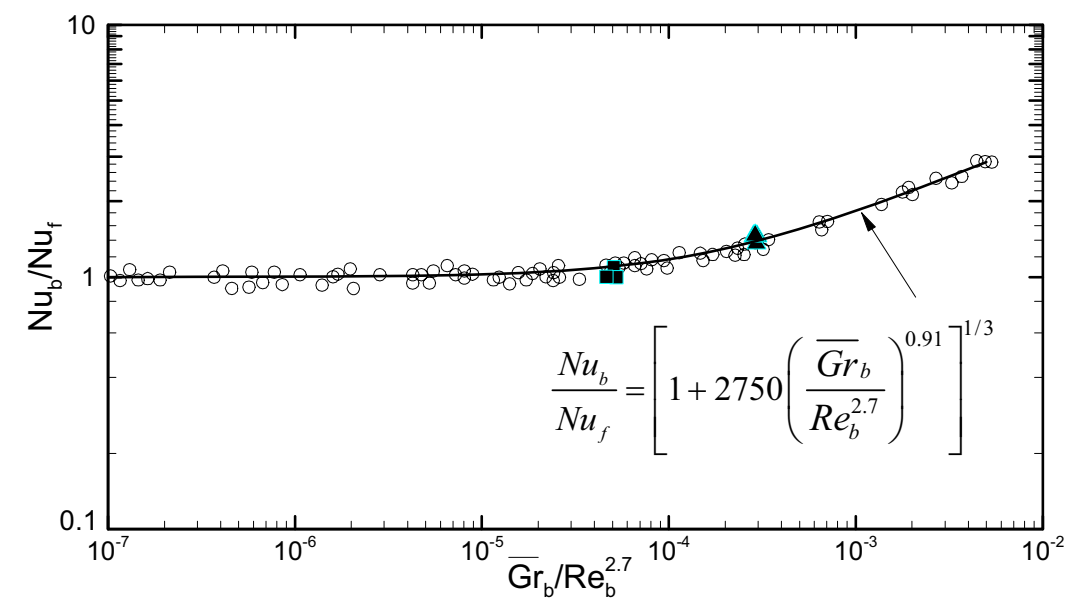

(b)

Fig. 2-6: Comparisons of the Nusselt number ratios between the present DNS and previous experiment for circular pipe flows. The present results are for the conditions listed in Table 2-1: (a) Upward flows: $\bullet$, case B; $\bullet$, case C; $\bullet$, case D; $\boldsymbol{\wedge}$, case G; $\boldsymbol{\nabla}$, case H; (b) downward flows: $\boldsymbol{\bullet}$, case E; $\boldsymbol{\Delta}$, case F. Open circles are experimental data of Fewser [see Jackson and Hall, 1979b]. Also shown in this figure are the recommended correlations [Jackson and Hall, 1979b] for mixed convection denoted by solid lines. 


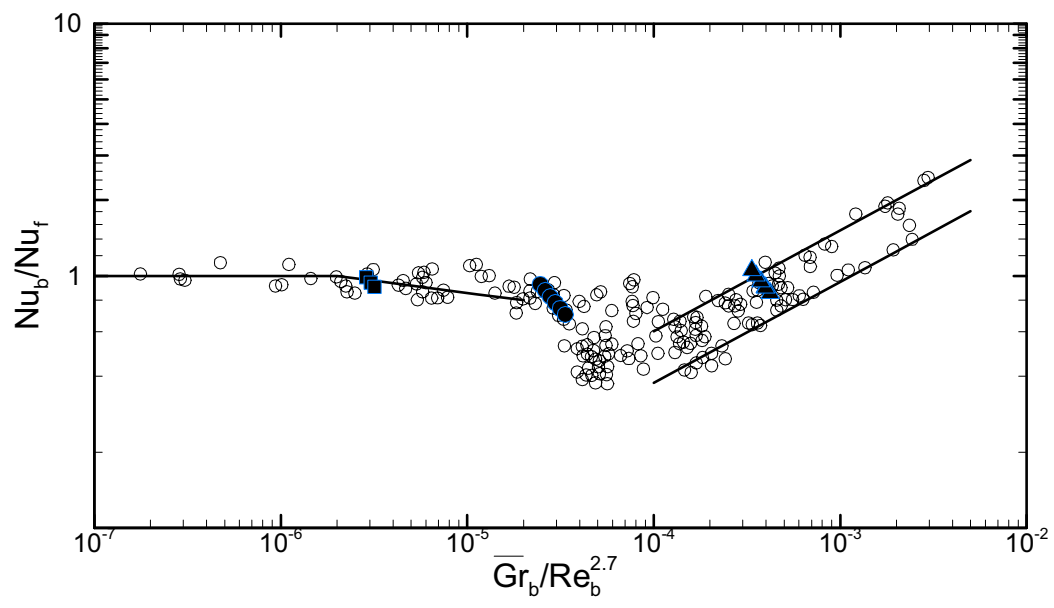

Fig. 2-7: Comparison of the Nusselt number ratios between the present DNS and previous experiment for circular pipe flows. The present results are for the conditions listed in Table 2-2: $\mathbf{-}$, case $\mathrm{X} ; \bullet \bullet$, case $\mathrm{Y}$; $\mathbf{\wedge}$, case $\mathrm{Z}$. Open circles are experimental data of Fewser [see Jackson and Hall, 1979b]. The flow is upward.

The changes of flow pattern in supercritical flows in a vertical pipe with strong buoyancy effects are investigated in Figure 2-8 where non-dimensional mean velocity and temperature distributions are compared between upward and downward flows for cases $\mathrm{C}$ and F, respectively. Here, the velocity and temperature are nondimensionalized with $U_{0}$ and $T_{0}$ at the inlet. Since the primary factor that causes the changes in flow pattern under the present conditions is the non-uniform temperature distribution with heat transfer, we need to examine the temperature distribution first. Although the imposed heat flux boundary conditions are the same for the two cases, the mean temperature distributions $\bar{T}(x, r)$ are quite different in upward and downward flows. In upward flow, $\bar{T}(x, r)$ shows that the near-wall fluid at $r / R \geq 0.95$ is heated to a highest temperature near $x / R=25.44$ and then it decreases further downstream. In the core region near the pipe centerline, however, $\bar{T}(x, r)$ increases continuously in the downstream direction. However, in downward flow, $\bar{T}(x, r)$ is shown to increase monotonically along and across the cross section. Compared to upward flow, its wall temperature $\left(T_{w}\right)$ at $r / R=1$ is lower, but the centerline temperature $\left(T_{c}\right)$ at $r / R=0$ is higher because heat transfer is enhanced to the central region by turbulence, as will be shown later. Because of this non-uniform temperature distribution, all the temperaturedependent properties of the fluid are also non-uniform in the cross section. For example, non-dimenisional mean density and enthalpy distributions are shown in Figure 2-9 where a very sharp decrease in the mean density is clearly visible near the heated wall. With such a large density change across the flow field, it is expected that the low density fluids near the wall are subjected to a very strong buoyancy force. In upward flow where buoyancy-induced fluid motion and forced flow occur in the same direction, the flow is highly accelerated near the wall while the centerline velocity is gradually reduced in the downstream direction as shown in the left column of Figure 2-8. Across the cross section at $x / R=25.04$ near where $T_{w}$ peak is observed (see Figure 2-5(a)), the deformation of the mean velocity profile evolves into an "M-shaped" one with $\partial U_{x} / \partial r \geq 0$ in the central region. In downward flow, however, the right column of Figure 2-8 shows that the flow in central region is more accelerated than in the near wall because the buoyancy induced motion acts against the flow direction. Petukhov and Polyakov [1988] refers to this kind of deformation of the mean velocity profile as the external effect of buoyancy. 

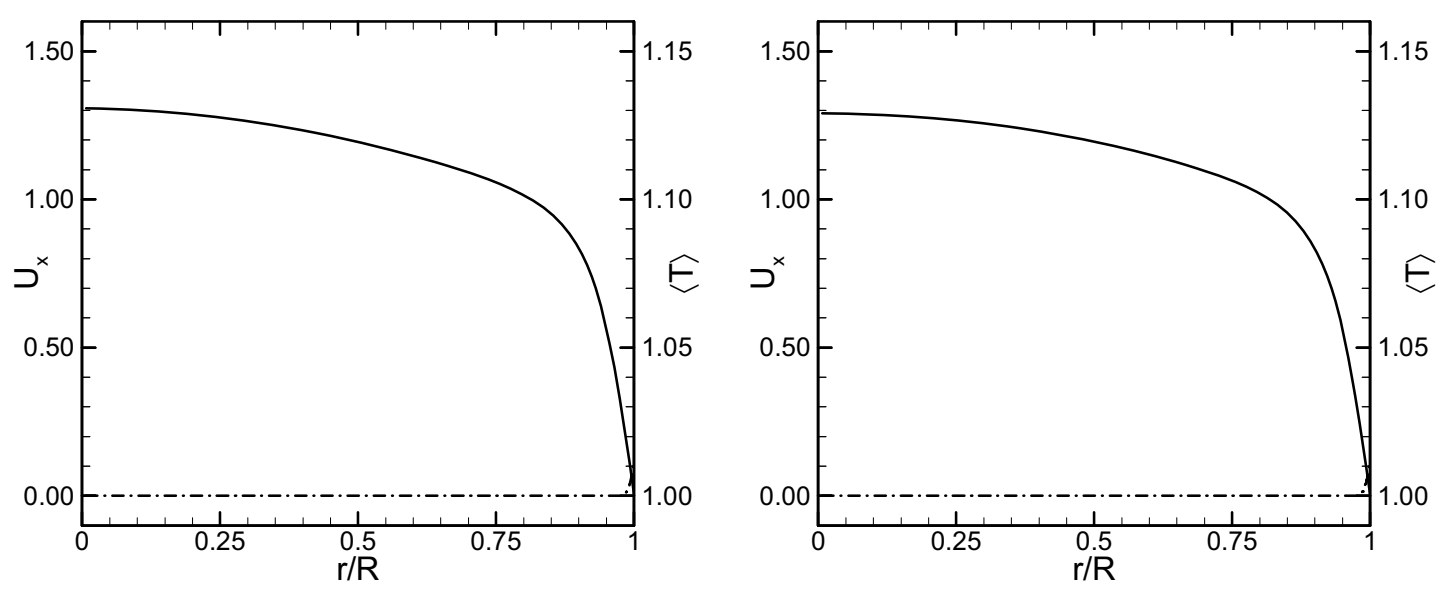

(a)
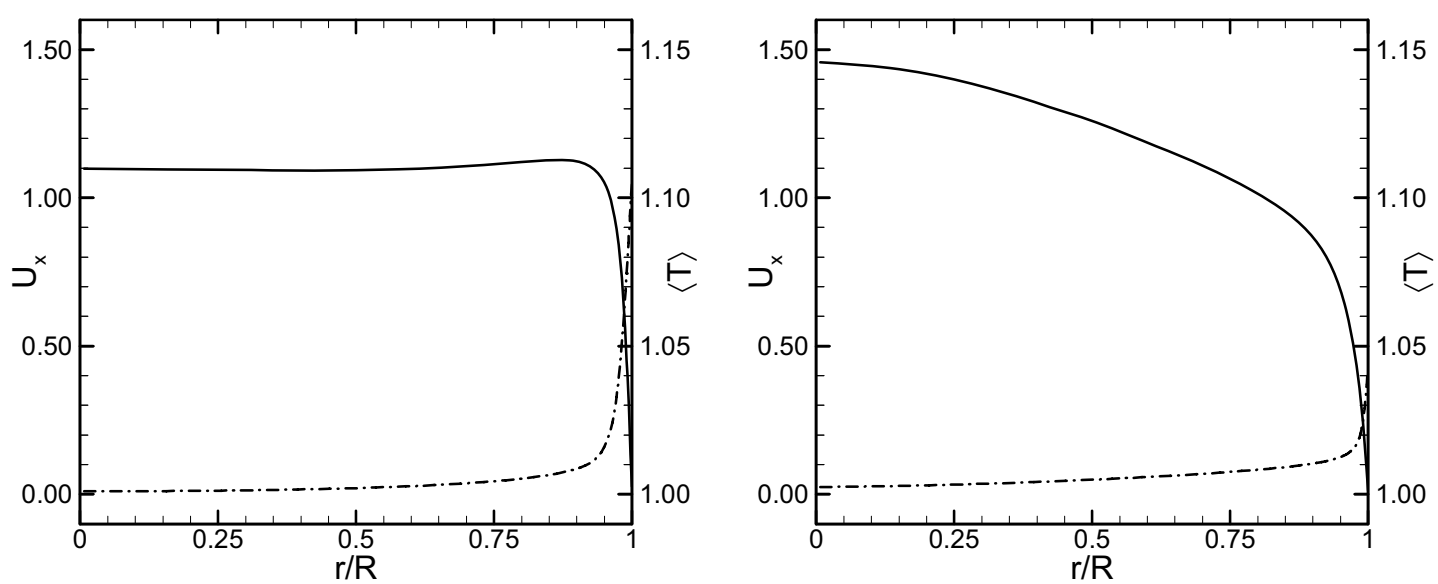

(b)
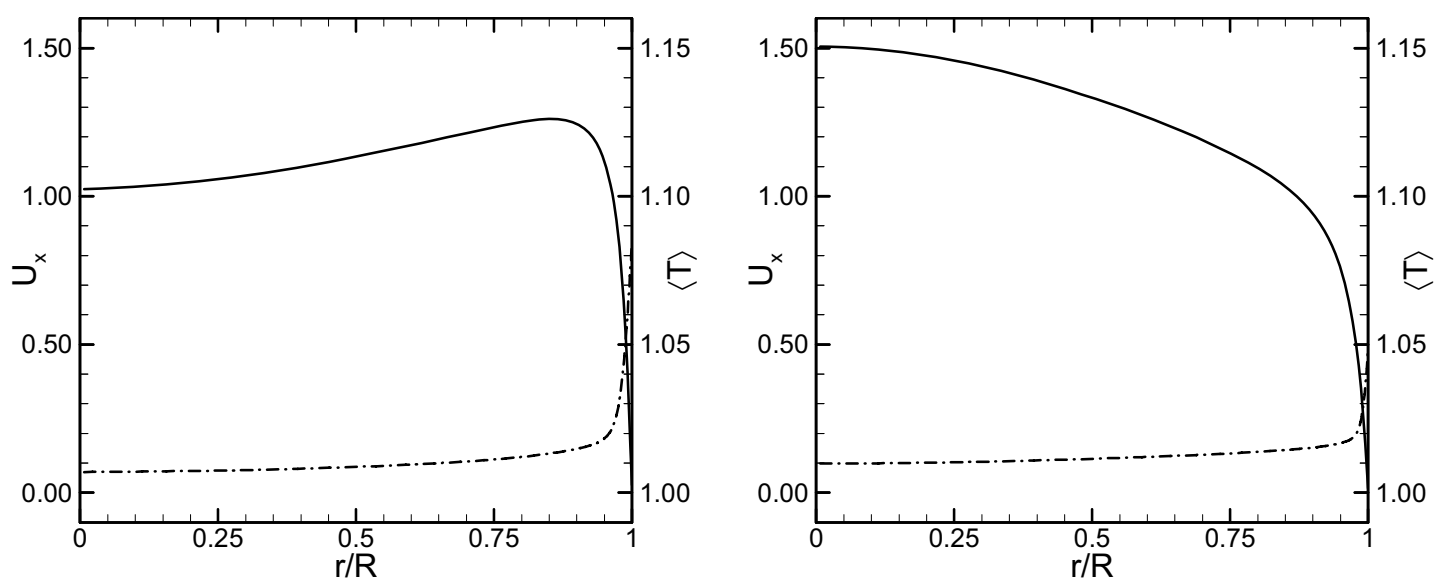

(c)

Fig. 2-8: Nondimensional mean velocity and temperature distributions for case $\mathrm{C}$, upward flow (left column) and case F, downward flow (right column): (a) $x / R=0.04$; (b) $x / R=25.04$; (c) $x / R=55.04$. - $U_{x}=\tilde{u}_{x} / U_{0}$ where $\tilde{u}_{x}=\overline{\rho u_{x}} / \bar{\rho} ;-\bullet-\bullet-,\langle T\rangle=\bar{T} / T_{0}$. 
We also observed that the buoyancy effects cause a considerable modification on turbulence statistics as well as on the mean velocity and temperature profiles. In Figure 2-10, we compare the turbulence kinetic energy (TKE) distributions for case $\mathrm{A}$, forced convection, and cases $\mathrm{C}$ and $\mathrm{F}$, mixed convection, for upward and downward flows, respectively. When the buoyancy effect is exactly zero as for case A, forced convection, the reduction in TKE is observed as shown in Figure 2-10(a); this observation can be easily explained if we consider the kinetic production of turbulence, $-\overline{\rho u_{i}^{\prime \prime \prime} u_{j}^{\prime \prime}}\left(\partial \tilde{u}_{i} / \partial x_{j}\right)$. This production term is expected to decrease in heated supercritical flows because

$$
-\overline{\rho u_{i}^{\prime \prime} u_{j}^{\prime \prime}} \frac{\partial \tilde{u}_{i}}{\partial x_{j}}=-\left(\overline{\rho u_{i}^{\prime \prime} u_{j}^{\prime \prime}}-\frac{1}{3} \overline{\rho u_{l}^{\prime \prime} u_{l}^{\prime \prime}} \delta_{i j}\right) \frac{\partial \tilde{u}_{i}}{\partial x_{j}}-\frac{1}{3} \overline{\rho u_{l}^{\prime \prime} u_{l}^{\prime \prime}} \frac{\partial \tilde{u}_{k}}{\partial x_{k}}
$$

where $\partial \tilde{u}_{i} / \partial x_{i}=-(1 / \bar{\rho})(D \bar{\rho} / D t)>0$ due to a significant density reduction of the fluid. This fact implies that a significant volume expansion of the supercritical-pressure fluid near the heated wall causes a reduction in the TKE distribution along the streamwise direction. In mixed convections with significant buoyancy effects, the predicted TKE distributions for cases $\mathrm{C}$ and $\mathrm{F}$ show considerable dependency on the direction of the buoyancy force. In upward flow, the predicted TKE distribution for case $\mathrm{C}$ shows a rapid reduction in the upstream region until $x / R=25.04$ before it increases substantially in the downstream region. As shown in Figures 2-5(a), the $T_{w}$ peak is observed near $x / R=25.04$ where the predicted TKE shows a minimum. In downward flow, however, the TKE distribution for case $\mathrm{F}$ shows a nearly monotonic increase in the flow direction. Other turbulence statistics also show a very complicated behavior depending on the direction of buoyancy force in mixed convection of supercriticalpressure fluids. These statistics are shown in Figure 2-11, where the radial profiles of the Reynolds normal and shear stresses, and the streamwise turbulent heat flux are compared between upward and downward flows for cases $\mathrm{C}$ and $\mathrm{F}$, respectively. Of particular interest in these figures is the observation of the sign changes in $\overline{\rho u_{x}^{\prime \prime} u_{r}^{\prime \prime}}$ and $\overline{\rho u_{x}^{\prime \prime} h^{\prime \prime}}$ for case C. The radial profile of $\overline{\rho u_{x}^{\prime \prime} u_{r}^{\prime \prime}}$ decreases continuously in the flow direction and becomes negative in the central region at $x / R \geq 25.04$ (Figure 2-11(b)). In the meanwhile, the quantity $\overline{\rho u_{x}^{\prime \prime} h^{\prime \prime}}$ shows a positive peak at $x / R=25.04$, preceded by an initial overall negative distribution at $x / R \leq 5.04$, and then it decreases slightly at further downstream while maintaining a positive distribution in most of the cross section (Figure 2-11(c)). Intuitively, these observed changes in the turbulence statistics are expected to occur because the mixing length theory postulates that it is more likely that $\overline{\rho u_{x}^{\prime \prime} u_{r}^{\prime \prime}}$ becomes negative and $\overline{\rho u_{x}^{\prime \prime} h^{\prime \prime}}$ becomes positive when the mean velocity profile deforms into an "M-shaped" one with predominant $\partial \tilde{u}_{x} / \partial r \geq 0$ in the cross section. As we have already shown in Figure 2-8, the so-called "M-shaped" mean velocity profile begins to form at $x / R \geq 25.04$ in upward flows where the peak velocity occurs near the wall due to buoyancy effect. This simple physical intuition indicates that the mean velocity profile changes due to buoyancy effects also affect the turbulence characteristics significantly. These complicated features of buoyancy effects on turbulence statistics may be best explained and quantitatively analyzed by means of the buoyancy production terms in the turbulence transport equations. However, we will not elaborate on this rather complex topic here (refer to the Topical Report [Bae et al., 2005, to appear in Physics of Fluids] for more details). However, it is worthwhile to point out here that the streamwise turbulent heat flux, $\overline{\rho u_{x}^{\prime \prime} h^{\prime \prime}}$, shown for case $\mathrm{C}$ in upward flow provides key information as to why such a sharp wall temperature peak exists in upward flows of supercritical-pressure fluids. As shown in Figures 2-11(c), turbulent advection of thermal energy occurs in the flow direction between $5.05 \leq x / R \leq 25.04$ because $\partial\left(\overline{\rho u_{x}^{\prime \prime} h^{\prime \prime}}\right) / \partial x>0$, which causes a further increase in $T_{w}$ in this region. Then the wall temperature decreases rapidly due to $\partial\left(\overline{\rho u_{x}^{\prime \prime} h^{\prime \prime}}\right) / \partial x<0$ at farther downstream region. This observation explains satisfactorily why such a sharp wall temperature peak is to be realized in upward supercritical flows and also explains why most turbulence models employed in the prediction of supercritical flows fail to capture this peculiar sharp $T_{w}$ peak accurately when the effects of density fluctuations (or the buoyancy production term) are not properly considered together with consistent treatment of the streamwise turbulent heat flux. Unfortunately, the effect of the buoyancy production term does not seem to be properly implemented into low-Reynolds-number turbulence models, as is clearly demonstrated by He et al. [2004]. 
In downward flows, however, a considerable enhancement of turbulence is observed for case $\mathrm{F}$ as shown in Figure 2-10(c) for TKE and in the right column of Figures $2-11(\mathrm{a})$ and (b) for $\overline{\rho u_{x}^{\prime \prime} u_{x}^{\prime \prime}}$ and $\overline{\rho u_{x}^{\prime \prime} u_{r}^{\prime \prime}}$, respectively. All these turbulence statistics are shown to be quite different from those for upward flow. The streamwise turbulent heat flux, $\overline{\rho u_{x}^{\prime \prime} h^{\prime \prime}}$, also shows no abnormal distribution in downward flows, which are overall negative as shown in Figure 2-11(c). As explained by Petukhov and Polyakov [1988], the buoyancy force in downward heated flows creates extra turbulence in the flow because the density distribution is intrinsically unstable $(g \partial \bar{\rho} / \partial x<0)$. This observation implies that the buoyancy effect provides an additional source of turbulence energy besides that due to the mean flow so that turbulence promotion is expected to occur in downward flows as can be attested for case F. According to Petukhov and Polyakov [1988], these effects of buoyancy on turbulence structure are referred to as the structural effect.

In order to visualize the structural effect of buoyancy, the instantaneous three-dimensional vortical structures are compared between upward and downward flows in Figure 2-12, where the iso-vorticity surfaces for $\omega_{x}= \pm 2.5$ are shown for cases $\mathrm{C}, \mathrm{H}$ and $\mathrm{F}$. The first two cases are for upward flows at $Q^{+}=1.2$ and 2.4, respectively, and the last one is for downward flow at $Q^{+}=1.2$. As expected, vortical motion of the fluids is reduced significantly in the upstream region for cases $\mathrm{C}$ and $\mathrm{H}$, where the flow is stabilized with buoyancy effect, but the strength of vortical motion increases again in the downstream region where turbulence begins to be restored. For case $\mathrm{H}$ at $Q^{+}=2.4$, this change occurs further upstream compared to case $\mathrm{C}$ at a lower heat flux condition. For case $\mathrm{F}$, downward flow, the vortical motion is significantly enhanced from the beginning of the heated section, as shown in Figure 2-12(c), because the buoyancy effect creates extra turbulence energy to promote turbulence.
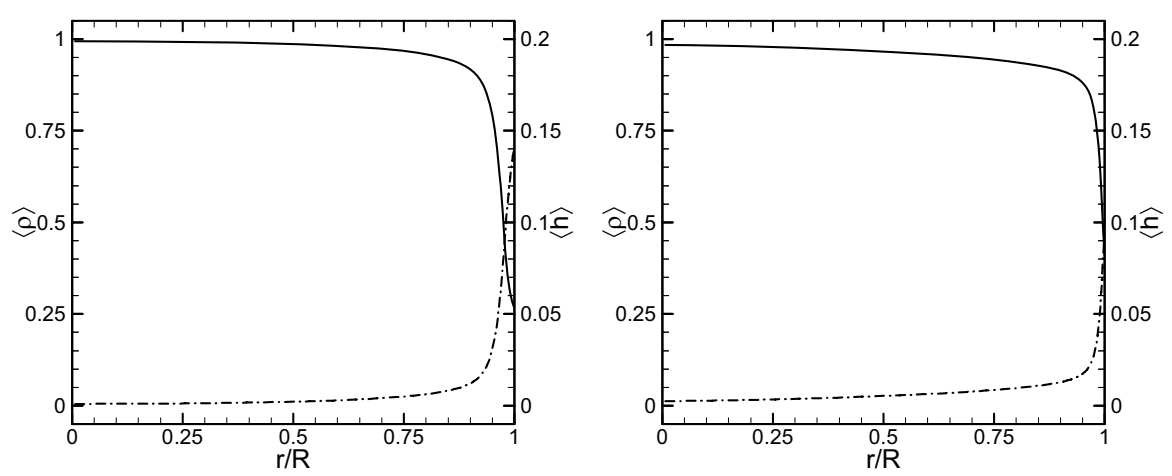

(a)
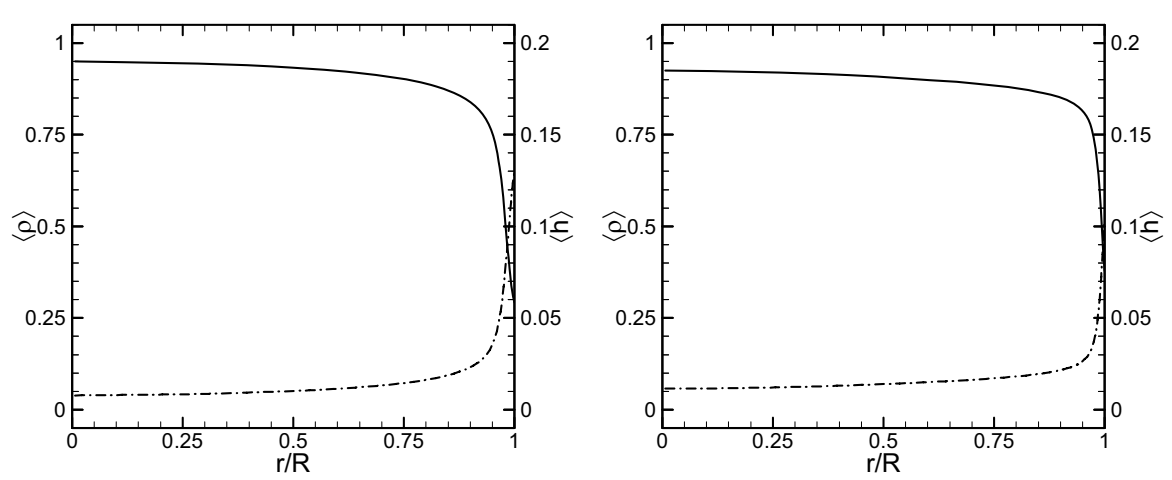

(b)

Fig. 2-9: Nondimensional mean density and static enthalpy distributions for case C, upward flow (left column) and case F, downward flow (right column): (a) $x / R=25.04$; (b) $x / R=55.04$.,$\langle\rho\rangle=\bar{\rho} / \rho_{0} ;-\bullet-\bullet-$, $\langle h\rangle=\left(\bar{h}-h_{0}\right) /\left(c_{p} T\right)_{0}$. 


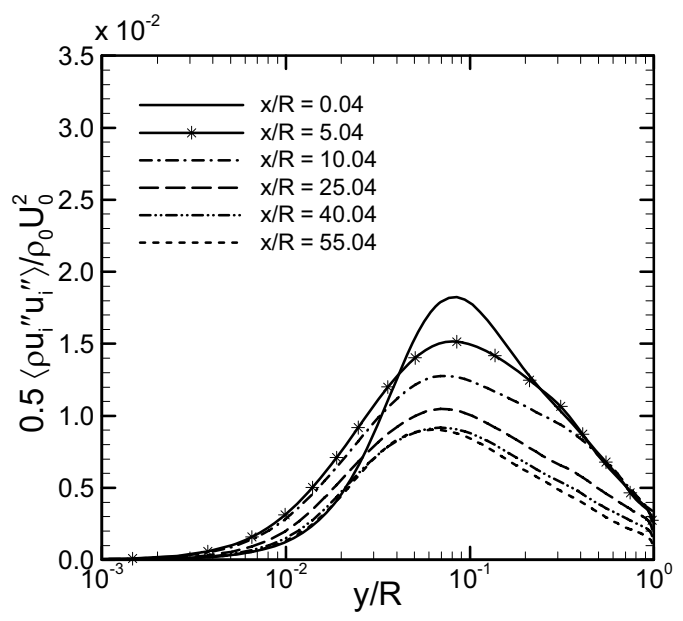

(a)

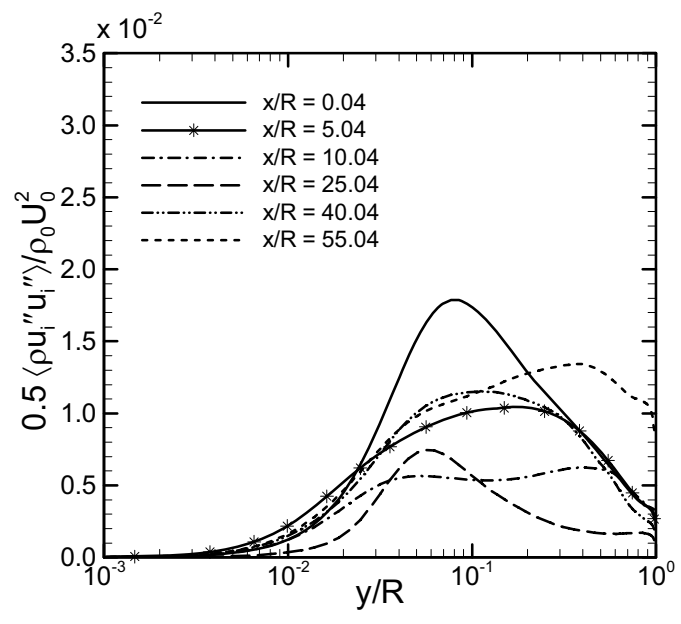

(b)

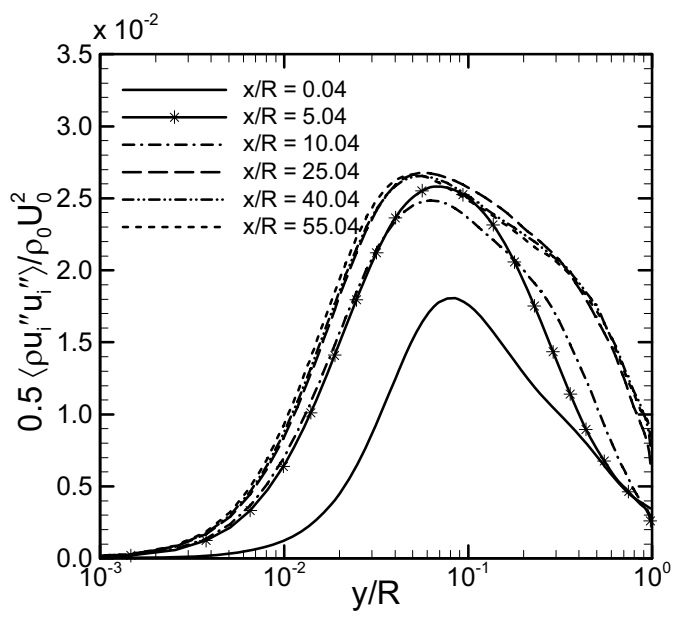

(c)

Fig. 2-10: Turbulent kinetic energy per unit volume, $(1 / 2)\left\langle\rho u_{i}^{\prime \prime} u_{i}^{\prime \prime}\right\rangle / \rho_{0} U_{0}^{2}$, where $\left\langle\rho u_{i}^{\prime \prime} u_{i}^{\prime \prime}\right\rangle=\left\langle\rho u_{x}^{\prime \prime} u_{x}^{\prime \prime}\right\rangle+\left\langle\rho u_{r}^{\prime \prime} u_{r}^{\prime \prime}\right\rangle+\left\langle\rho u_{\theta}^{\prime \prime} u_{\theta}^{\prime \prime}\right\rangle$ and $u_{i}^{\prime \prime}=u_{i}-\tilde{u}_{i}$ : (a) case A, forced convection; (b) case C, upward flow; (c) case $\mathrm{F}$, downward flow. In this figure the radial profiles are plotted against $y / R=1-r / R$ in log-scale. 

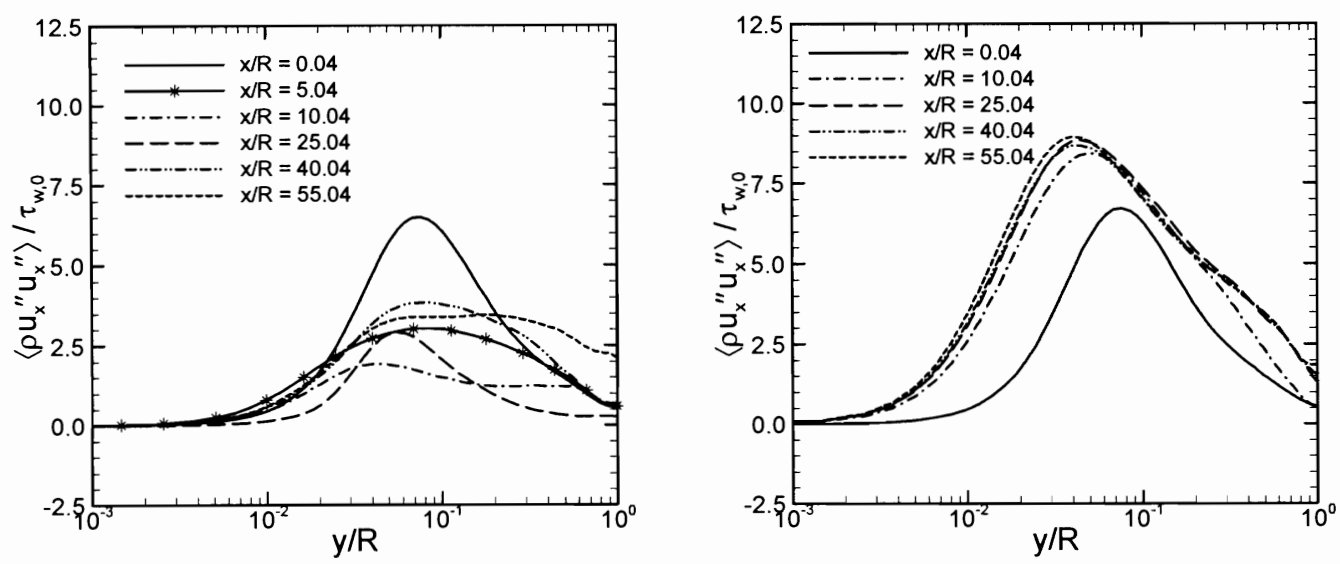

(a)
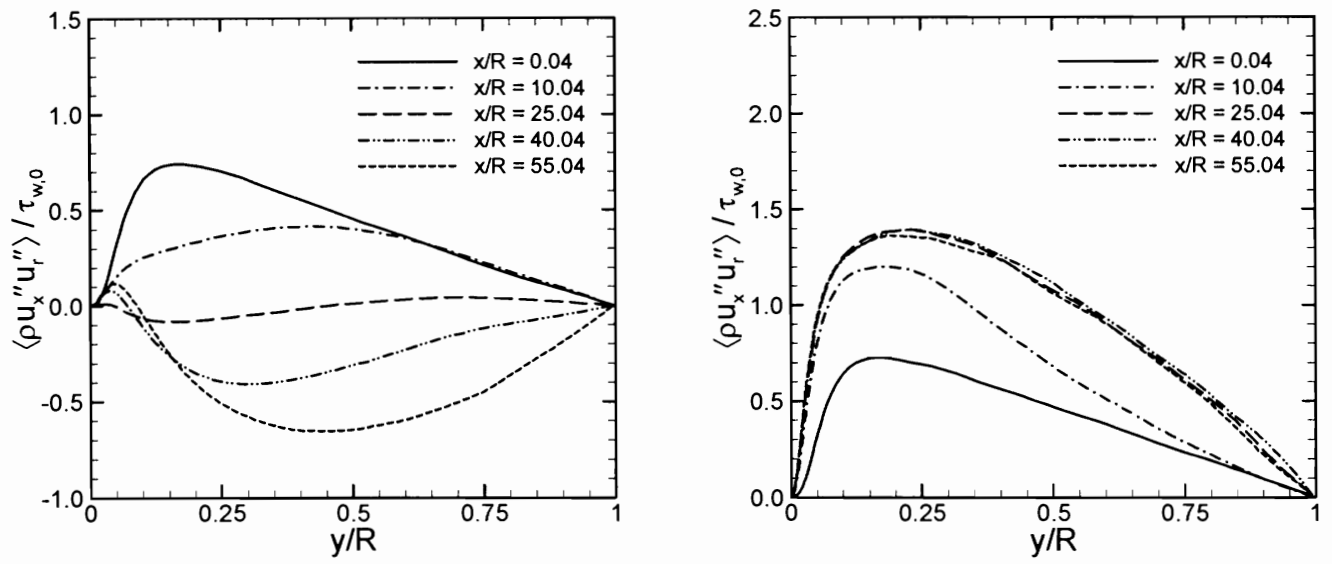

(b)
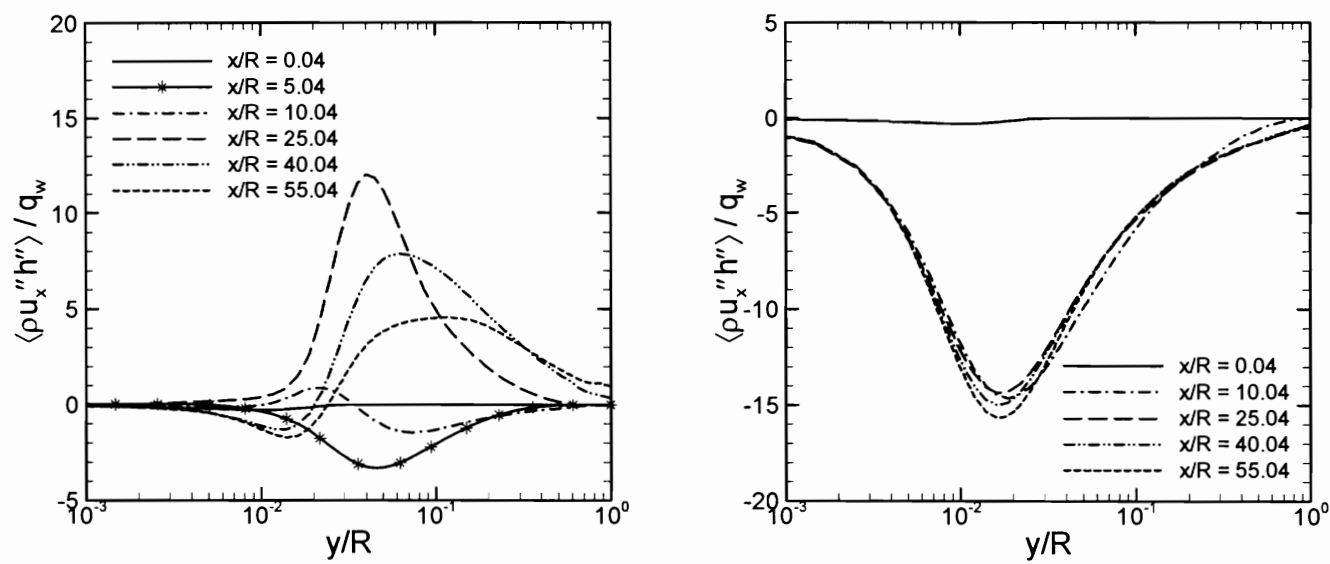

(c)

Fig. 2-11: Reynolds stress and streamwise turbulent heat flux distributions for case C, upward flow (left column) and case F, downward flow (right column): (a) $\left\langle\rho u_{x}^{\prime \prime} u_{x}^{\prime \prime}\right\rangle / \tau_{w, 0} ;$ (b) $\left\langle\rho u_{x}^{\prime \prime} u_{r}^{\prime \prime}\right\rangle / \tau_{w, 0} ;$ (c) $\left\langle\rho u_{x}^{\prime \prime} h^{\prime \prime}\right\rangle / q_{w}$. The quantities $\tau_{w, 0}$ and $q_{w}$ represent the inlet wall shear stress and the imposed heat flux at the wall, respectively. 


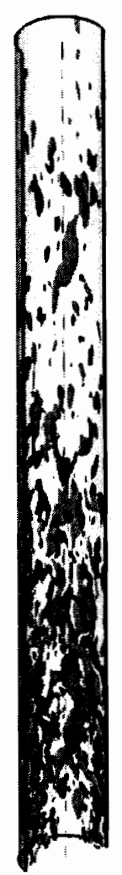

0 10D

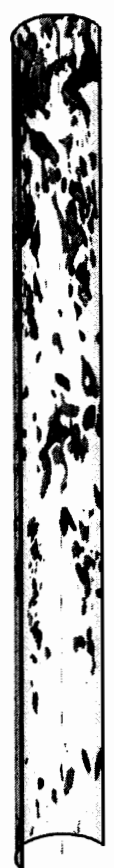

10 20D

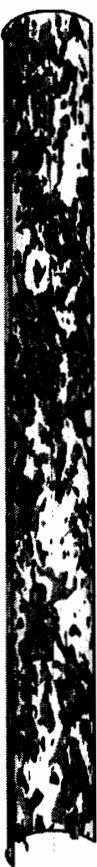

20 30D

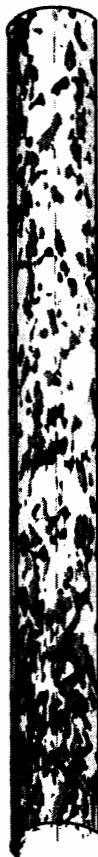

0 10D

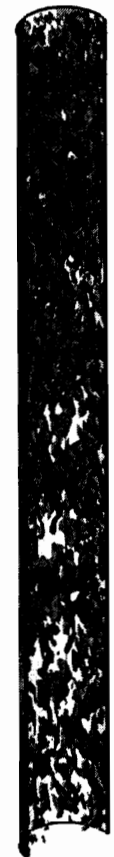

10 20D

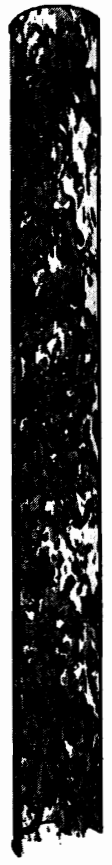

20 30D

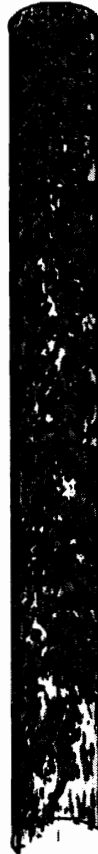

0 10D

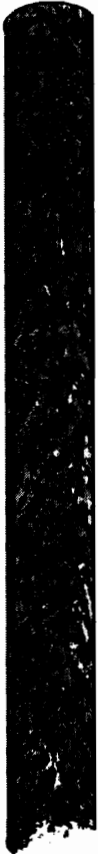

10-20D

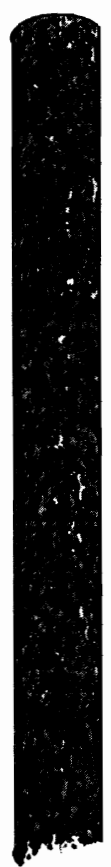

20-30D

(a)

(b)

(c)

Fig. 2-12: Instantaneous iso-vorticity surfaces for $\omega_{x}= \pm 2.5$ : (a) case C, upward flow at $Q^{+}=1.2$; (b) case $\mathrm{H}$, upward flow at $Q^{+}=2.4$; (c) case F, downward flow at $Q^{+}=1.2$. Dark gray, $\omega_{x}=-2.5$; light gray, $\omega_{x}=+2.5$.

DNS of vertical annular channel flows of supercritical-pressure $\mathrm{CO}_{2}$ Influences of strongly varying properties of supercritical-pressure $\mathrm{CO}_{2}$ on turbulent convective heat transfer are also investigated for annular channel flows. We consider thermally-developing flows in a vertical annular channel where the inner wall is heated with a constant heat flux and the outer wall is insulated. As in the pipe flows, $\mathrm{CO}_{2}$ is chosen as the working fluid at a pressure of $8 \mathrm{MPa}$. Only upward flows are considered. Figure 2-13 shows the schematic diagram of the flow region and the boundary conditions for annular flows. The inner-to-outer wall radius ratio is $r_{\mathrm{i}} / r_{\mathrm{o}}=0.5$. Only a quarter sector of the full annular cross section is considered in the present study to maintain adequate grid resolution near the outer wall region. The domain size of the inflow generator is chosen as $L / \delta=5 \pi$ while that of the main heated region is $30 \pi$, for which a $769 \times 69 \times 129$ grid is used. The $\delta$ denotes the channel half width. A grid resolution study is also conducted with node spacing twice as large in the radial direction to confirm the adequacy of the present solutions obtained with a baseline grid.

The studies of annular flows are particularly relevant to technology advancement for nuclear reactor thermal hydraulics since the flow passages or the cooling channel geometries in many advanced nuclear reactor designs are composed of various types of sub-channels with complex, non-circular geometries [McEligot et al., 2004; Cheng et al., 2003]. The studies of an annular flow with heat transfer from a heated inner rod may provide useful information on heat transfer through boundary layers with convex curvature which is frequently found in many sub-channel geometries of the advanced nuclear reactor systems. 


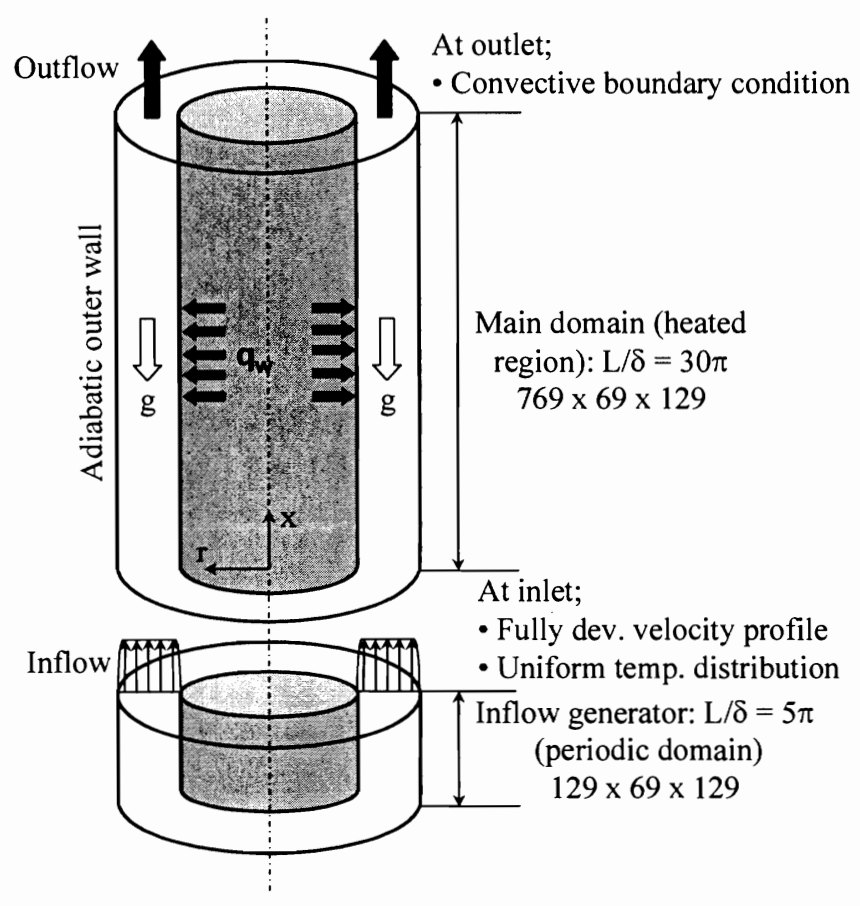

Fig. 2-13: Schematic diagram of the flow region and boundary conditions for upward annular channel flows

Table 2-3 shows the flow conditions considered in the present study. The inlet Reynolds number is $\operatorname{Re}_{0}\left(=\rho_{0} U_{0} D_{h} / \mu_{0}\right)=8900$ based on the hydraulic diameter $\left(D_{h}=4 \delta\right)$ and the inlet bulk velocity $U_{0}$. Three heating conditions of $Q^{+}\left(=q_{w} \delta / k_{0} T_{0}\right)=0.25,1.20$ and 2.40 are investigated at the inlet temperatures of $T_{0}=$ $301.15 \mathrm{~K}$ and $358.15 \mathrm{~K}$. The table also summarizes the buoyancy parameters in terms of $G r_{0}^{*}\left(=g \beta_{0} q_{w} D_{h}^{4} / v_{0}^{2} k_{0}\right)$, $B o\left(=G r_{0}^{*} / \operatorname{Re}_{0}^{3.425} \operatorname{Pr}_{0}^{0.8}\right)$ and $\overline{G r}_{b} / \operatorname{Re}_{b}^{2.7}$. Type CP refers to the constant-property solutions obtained with fluid properties fixed at the inlet values and FC denotes variable-property forced convection without buoyancy effect. Mixed convection with significant buoyancy effects is labeled as MC in the table.

Figures 2-14 through 2-17 show the predicted non-dimensional wall temperature and the local Nusselt number distributions at the heated inner surface for $Q^{+}=0.25,1.20$ and 2.40, respectively. For each heating condition, the constant-property solution (CP) and the variable-property solutions for forced convection (FC) and mixed convection (MC) are compared with each other in order to ascertain the effects of strongly-varying properties of supercritical-pressure $\mathrm{CO}_{2}$ and the buoyancy effect. Also included in these figures are the predictions obtained from the modified Krasnoschekov and Protopopov correlation [see Jackson and Hall, 1979a] for fully-established, forced convection to supercritical-pressure fluids. 
Table 2-3: Flow conditions for vertical annular channel flows of supercritical-pressure $\mathrm{CO}_{2}$ at $P_{0}=8 \mathrm{MPa}$ and $R e_{0}=8900$. The inner-to-outer wall radius ratio of the annular channel is $r_{i} / r_{o}=0.5$ and $\delta$ denotes a channel half width defined as $\delta=0.5\left(r_{0}-r_{i}\right)$.

\begin{tabular}{|c|c|c|c|c|c|c|c|c|c|c|c|c|}
\hline Case & Type & Dir. & $\begin{array}{c}D_{h}(=4 \delta) \\
{[\mathrm{mm}]}\end{array}$ & $\begin{array}{l}T_{0} \\
{[\mathrm{~K}]}\end{array}$ & $\begin{array}{c}G_{0}\left(=\rho_{0} U_{0}\right) \\
{\left[\mathrm{kg} / \mathrm{m}^{2} \mathrm{~s}\right]}\end{array}$ & $P r_{0}$ & $\begin{array}{c}Q^{+} \\
\left(q_{w} \delta / k_{0} T_{0}\right)\end{array}$ & $\begin{array}{c}q^{+} \\
\times 10^{4}\end{array}$ & $\begin{array}{c}q_{w} \\
{\left[\mathrm{~kW} / \mathrm{m}^{2}\right]}\end{array}$ & $\begin{array}{l}G r_{0}^{*} \\
\times 10^{-8}\end{array}$ & $\begin{array}{r}B o \\
\times 10^{6}\end{array}$ & $\begin{array}{c}\left(\overline{G r}_{b} / R e_{b}^{2.7}\right)^{a} \\
\times 10^{4}\end{array}$ \\
\hline $\mathrm{C} 1$ & $\mathrm{CP}$ & - & 2.0 & 301.15 & 274.62 & 2.48 & 0.25 & 0.365 & 12.86 & 0 & 0 & 0 \\
\hline $\mathrm{F} 1$ & FC & - & 2.0 & 301.15 & 274.62 & 2.48 & 0.25 & 0.365 & 12.86 & 0 & 0 & 0 \\
\hline $\begin{array}{l}\text { M1 } \\
\text { C2 }\end{array}$ & $\begin{array}{c}\mathrm{MC} \\
\mathrm{CP}\end{array}$ & $\begin{array}{l}\text { Up } \\
-\end{array}$ & $\begin{array}{l}2.0 \\
2.0\end{array}$ & $\begin{array}{l}301.15 \\
301.15\end{array}$ & $\begin{array}{l}274.62 \\
274.62\end{array}$ & $\begin{array}{l}2.48 \\
2.48\end{array}$ & $\begin{array}{l}0.25 \\
1.20\end{array}$ & $\begin{array}{l}0.365 \\
1.752\end{array}$ & $\begin{array}{l}12.86 \\
61.74\end{array}$ & $\begin{array}{c}0.700 \\
0\end{array}$ & $\begin{array}{c}0.846 \\
0\end{array}$ & $\begin{array}{c}0.205 \\
0\end{array}$ \\
\hline $\mathrm{F} 2$ & FC & - & 2.0 & 301.15 & 274.62 & 2.48 & 1.20 & 1.752 & 61.74 & 0 & 0 & 0 \\
\hline $\begin{array}{l}\text { M2 } \\
\text { C3 }\end{array}$ & $\begin{array}{l}\text { MC } \\
\text { CP }\end{array}$ & $\begin{array}{l}\text { Up } \\
-\end{array}$ & $\begin{array}{l}2.0 \\
2.0\end{array}$ & $\begin{array}{l}301.15 \\
301.15\end{array}$ & $\begin{array}{l}274.62 \\
274.62\end{array}$ & $\begin{array}{l}2.48 \\
2.48\end{array}$ & $\begin{array}{l}1.20 \\
2.40\end{array}$ & $\begin{array}{l}1.752 \\
3.504\end{array}$ & $\begin{array}{c}61.74 \\
123.48\end{array}$ & $\begin{array}{c}3.358 \\
0\end{array}$ & $\begin{array}{c}4.061 \\
0\end{array}$ & $\begin{array}{c}1.685 \\
0\end{array}$ \\
\hline F3 & FC & - & 2.0 & 301.15 & 274.62 & 2.48 & 2.40 & 3.504 & 123.48 & 0 & 0 & 0 \\
\hline $\begin{array}{c}\text { M3 } \\
\text { F4 }\end{array}$ & $\begin{array}{c}\text { MC } \\
\text { FC }\end{array}$ & $\begin{array}{c}\text { Up } \\
-\end{array}$ & $\begin{array}{l}2.0 \\
2.0\end{array}$ & $\begin{array}{l}301.15 \\
358.15\end{array}$ & $\begin{array}{c}274.62 \\
92.09\end{array}$ & $\begin{array}{l}2.48 \\
1.02\end{array}$ & $\begin{array}{l}2.40 \\
2.40\end{array}$ & $\begin{array}{l}3.504 \\
10.53\end{array}$ & $\begin{array}{c}123.48 \\
49.80\end{array}$ & $\begin{array}{c}6.717 \\
0\end{array}$ & $\begin{array}{c}8.122 \\
0\end{array}$ & $\begin{array}{c}1.882 \\
0\end{array}$ \\
\hline M4 & $\mathrm{MC}$ & Up & 2.0 & 358.15 & 92.09 & 1.02 & 2.40 & 10.53 & 49.80 & 1.017 & 2.966 & 0.264 \\
\hline
\end{tabular}

a The values for $\overline{G r}_{b} / \operatorname{Re}_{b}^{2.7}$ shown in the table are the maximum value at each condition.

As shown in Figure 2-14, the DNS results for case F1 are reasonably well matched with the correlation at low $Q^{+}$where the wall-to-bulk temperature difference is very small $\left(\Delta T=T_{w}-T_{b} \approx 6 \mathrm{~K}\right)$ and $T_{w}<T_{p c}$. Because of this small difference in temperature, the influences of variable-properties in forced convection are not

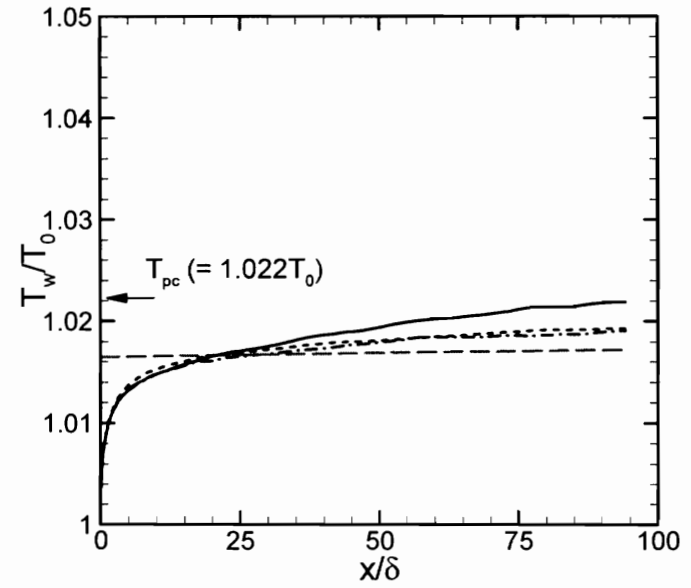

(a)

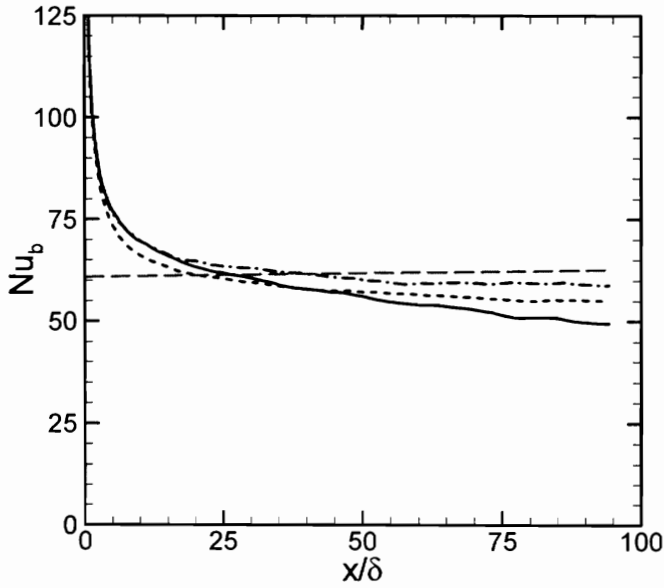

(b)

Fig. 2-14: Wall temperature and the local Nusselt number distributions along the heated inner wall at $Q^{+}=0.25$, where $T_{b}<T_{w}<T_{p c}$ : (a) $T_{w} / T_{0}$; (b) $N u_{b} \ldots-$ - - , case C1, constant-property; -•-•-, case F1, forced convection; - case M1, mixed convection; ----, modified Krasnoschekov and Protopopov correlation for fully-established forced convection.

expected to be significant at this low heating condition. A slight increase in $N u_{b}$ for case $\mathrm{F} 1$ over the results for case $\mathrm{C} 1$ is because the bulk thermal conductivity $\left(k_{b}=k_{b}\left\{T_{b}\right\}\right)$ decreases slightly as $T_{b}$ increases in the flow direction. However, the predicted results for case M1 show a measurable reduction in $N u_{b}$ (more than $15 \%$ ) compared to the buoyancy-free forced convection for case F1, indicating that even at this low heating condition the buoyancy effect is not negligible. With experimental data for air and water at atmospheric pressure flowing in heated annuli, Jackson 
[2005] has shown that about a ten per cent reduction in heat transfer coefficient occurs due to the buoyancy effect at $B o \approx 5 \times 10^{-7}$ and the present results for case $\mathrm{M} 1$ at $B o \approx 8.5 \times 10^{-7}$ seem to be very consistent with the experimental data.

Figures 2-15 and 2-16 show the results at relatively high heating conditions where the condition of $T_{b}<T_{p c}<T_{w}$ is met. Due to strong dependence of physical properties on temperature at these conditions, the energy equation becomes highly non-linear so that the proportionality between the heat flux and temperature difference no longer exists. With increase in heat flux, the buoyancy effect also increases and the combined effects of variable property and buoyancy cause marked differences in heat transfer to supercritical-pressure fluids. Previous experimental data show that even for forced convection without significant buoyancy effects, a considerable impairment in the heat transfer coefficient was observed at this condition [Shiralkar and Griffith, 1970]. Furthermore, none of the existing correlations is known to provide reasonably accurate predictions for heat transfer coefficient at this condition. This situation is rather clearly shown in Figures 2-15(b) and 2-16(b) where the Krasnoschekov and Protopopov correlation (which is considered as the best for forced convection heat transfer to supercritical-pressure fluids) overpredicts the Nusselt number significantly as compared to the present results for cases F2 and F3. In fact, these results for forced convection without buoyancy effects are qualitatively similar to the experimental data of Shiralkar and Griffith [1970] where a broad wall temperature peak is observed regardless of the flow direction, as also shown in the predicted wall temperature distribution for case F3 at $Q^{+}=2.4$ near the end of the heated region. In the case of mixed convection, however, the results for cases M2 and M3 indicate that the buoyancy effect causes a rapid rise in $T_{\mathrm{w}}$ in the upstream region which is followed by a large drop of $T_{w}$ in the downstream region after the peak. Consequently, a non-monotonic wall temperature distribution develops in mixed convection where the predicted wall temperature peak is sharper and is located further upstream than for forced convection. Before the peak, the local Nusselt number for cases M2 and M3 are shown to be lower than those for cases F2 and F3, but in the downstream region of the peak the opposite happens so that mixed convection shows better performance than forced convection. Indeed, these characteristics are qualitatively very similar to our previous results for supercriticalpressure $\mathrm{CO}_{2}$ flowing upward in a vertical circular pipe [Bae et al, 2005].

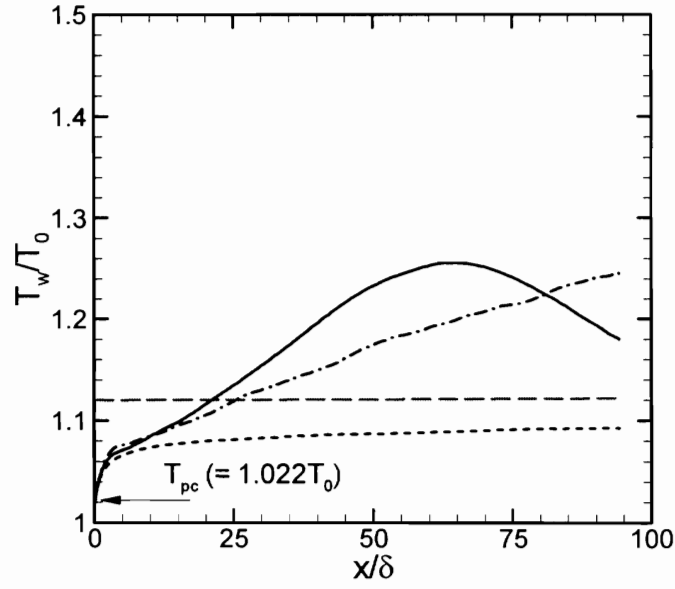

(a)

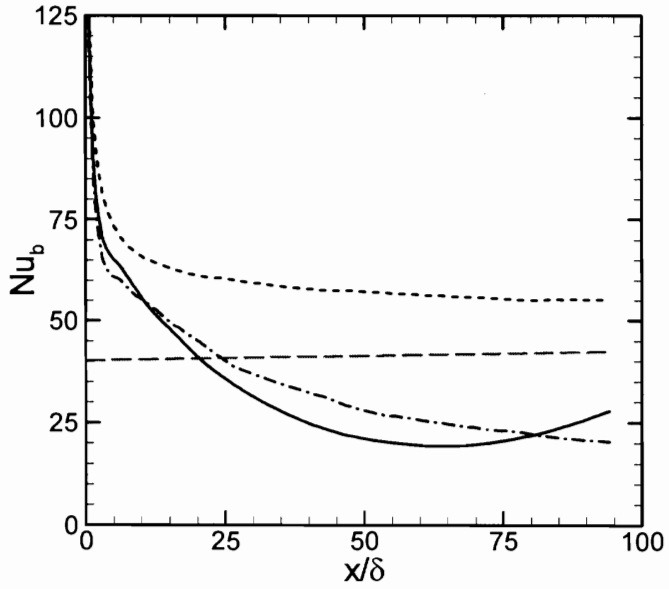

(b)

Fig. 2-15: Wall temperature and the local Nusselt number distributions along the heated inner wall for $Q^{+}=1.20$, where $T_{b}<T_{p c}<T_{w}$ : (a) $T_{w} / T_{0}$; (b) $N u_{b} \ldots-$ - - , case C2, constant-property; -•-•-, case F2, forced convection; - case M2, mixed convection; ----, modified Krasnoschekov and Protopopov correlation for fully-established forced convection. 


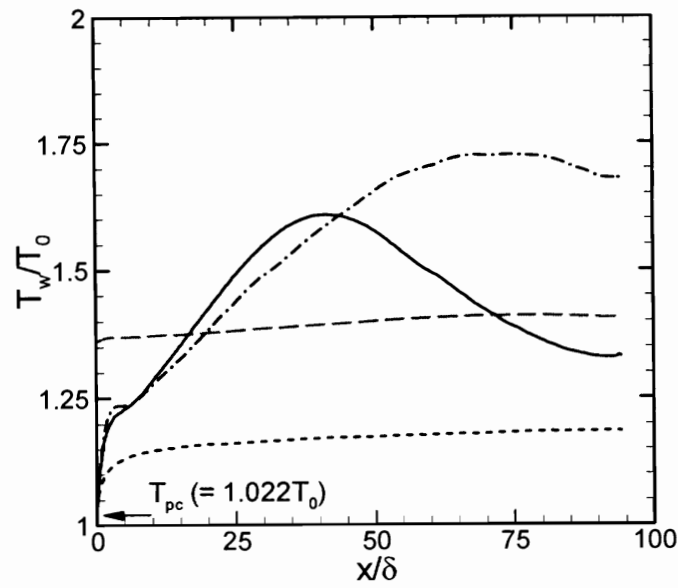

(a)

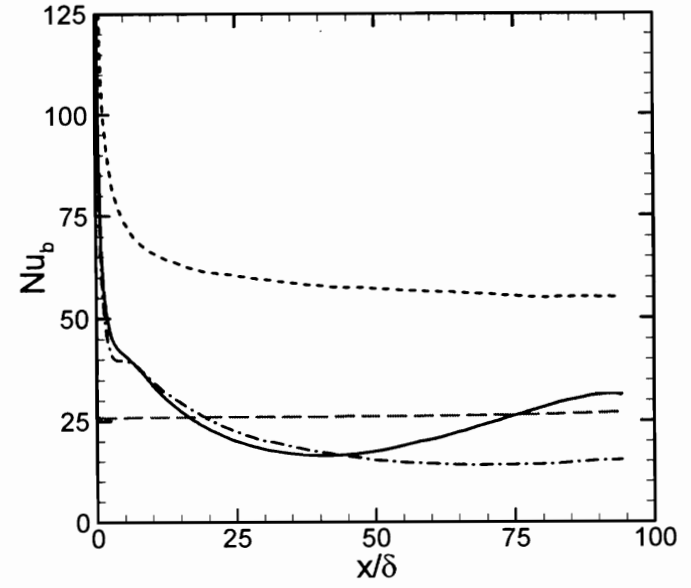

(b)

Fig. 2-16: Wall temperature and the local Nusselt number distributions along the heated inner wall for $Q^{+}=2.40$,

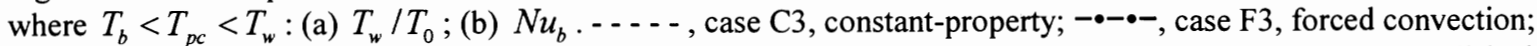
$\longrightarrow$, case M3, mixed convection; ----, modified Krasnoschekov and Protopopov correlation for fully-established forced convection.

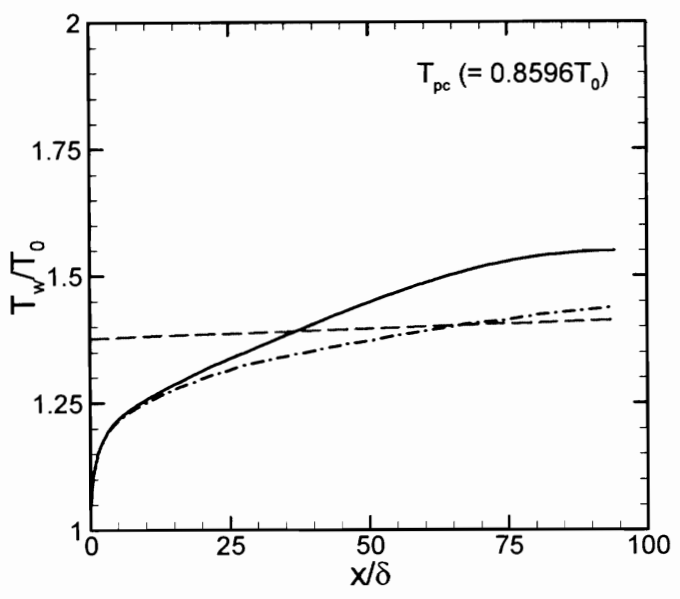

(a)

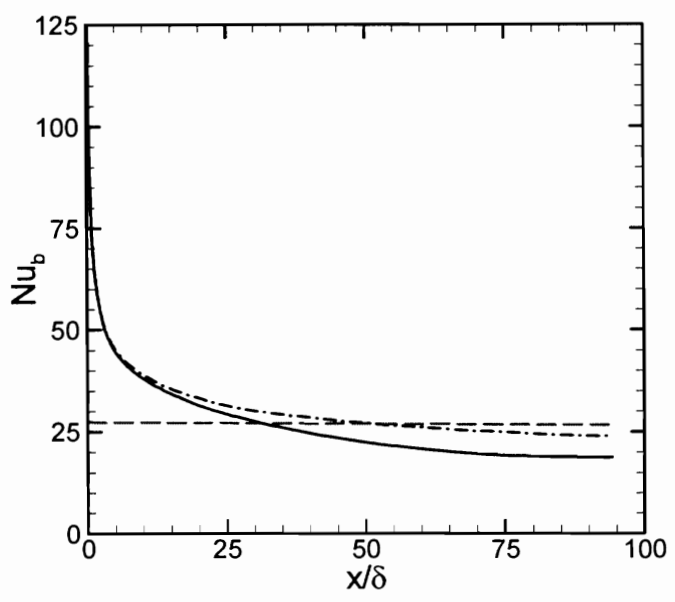

(b)

Fig. 2-17: Wall temperature and the local Nusselt number distributions along the heated inner wall for $Q^{+}=2.40$,

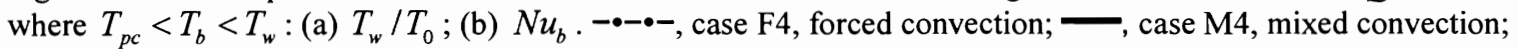

, modified Krasnoschekov and Protopopov correlation for fully-established forced convection.

In Figure 2-17, the results for cases F4 and M4 are compared in terms of the wall temperature and the local Nusselt number distributions. For these conditions, the inlet temperature is set to $T_{0}=385.15 \mathrm{~K}$ which is well above the pseudo-critical temperature so that the fluid temperatures are in the range of $T_{p c}<T_{b}<T_{w}$ where the supercritical-pressure $\mathrm{CO}_{2}$ is expected to show a gas-like behavior. As shown in Figure 2-17(b), the predicted $N u_{b}$ distribution for case F4 is in good agreement with the Krasnoschekov and Protopopov correlation near the end of the heated section where the flow is almost quasi-fully-developed. Again in this condition, the buoyancy effect is 
shown to cause a measurable reduction in Nusselt number for case M4 at $B o=2.97 \times 10^{-6}$ compared to forced convection.

Figure 2-18 shows the mean velocity distributions for cases F2 and M2, where the forced and the mixed convection results at $Q^{+}=1.20$ are compared at five locations along the streamwise direction. The profile at $x / \delta=0$ corresponds to the mean velocity profile for constant-property, fully-developed flow at the inlet. As clearly shown in both subfigures, local flow acceleration occurs near the heated inner wall in variable-property, supercritical flow due to thermal expansion and buoyancy effect, both of which are caused by a significant reduction of density.

Furthermore, the local flow acceleration is considerably larger with buoyancy effects so that the mean velocity

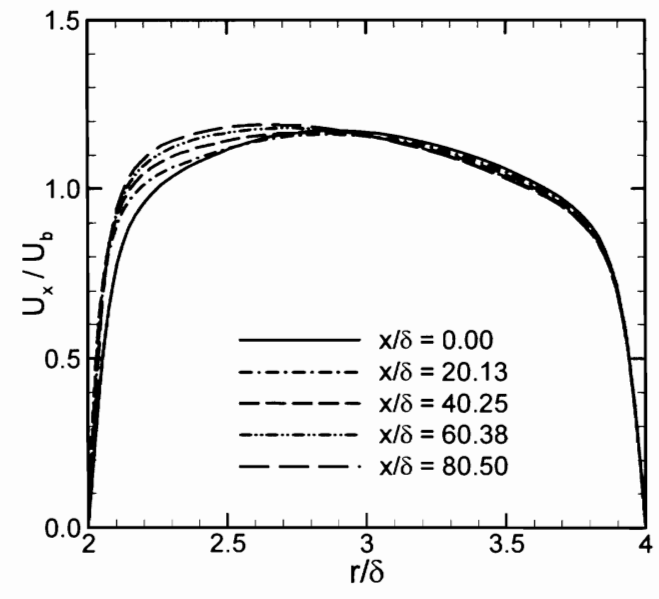

(a)

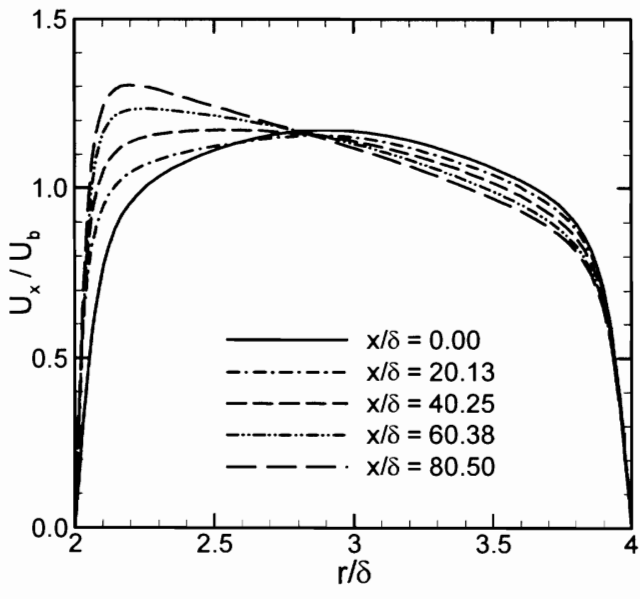

(b)

Fig. 2-18: Mean velocity profiles at $Q^{+}=1.20$ : (a) case F2, forced convection; (b) case M2, mixed convection. The velocity $U_{x}$ shown in the figure is the Favre-averaged mean velocity defined as $U_{x}\left(=\tilde{u}_{x}\right)=\overline{\rho u_{x}} / \bar{\rho}$.

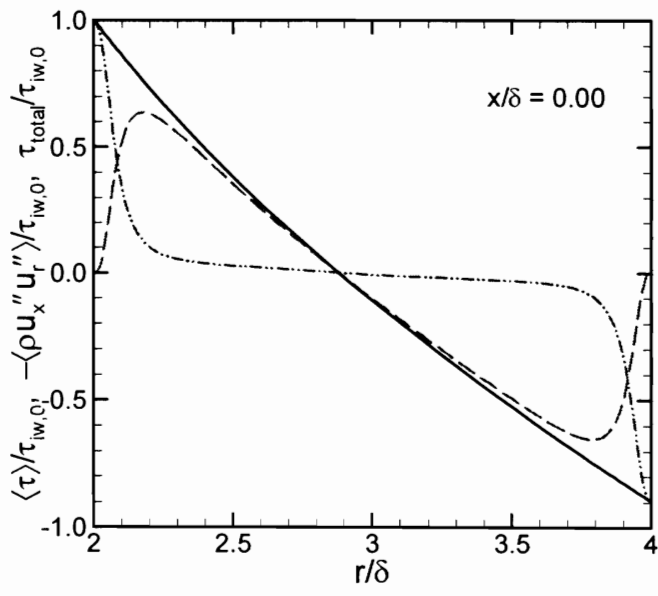

(a)

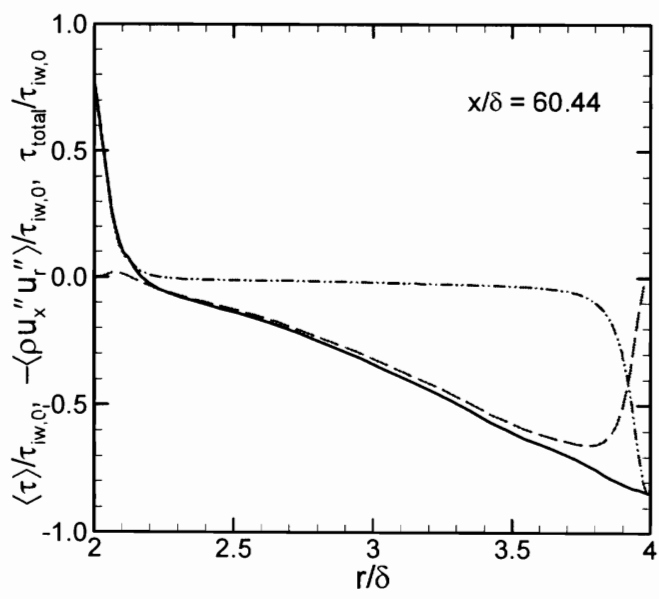

(b)

Fig. 2-19: Normalized shear stress profiles for case M2, mixed convection: (a) $x / \delta=0.00$, the inlet; (b) $x / \delta=60.44 . \cdots-\cdots-,\langle\tau\rangle=\bar{\mu} / \operatorname{Re}\left(\partial \bar{u}_{x} / \partial r+\partial \bar{u}_{r} / \partial x\right) ;----,\left\langle\rho u_{x}^{\prime \prime} u_{r}^{\prime \prime}\right\rangle ;-$, $\tau_{\text {total }}=\bar{\mu} / \operatorname{Re}\left(\partial \bar{u}_{x} / \partial r+\partial \bar{u}_{r} / \partial x\right)-\left\langle\rho u_{x}^{\prime \prime} u_{r}^{\prime \prime}\right\rangle$. Each component of shear stress in the figure is normalized with the inner wall shear stress at the inlet $\left(\tau_{i w, 0}\right)$. 
profile becomes more asymmetric as the flow progresses downstream as shown in Figure 2-18(b). Along with the changes in the mean velocity profiles, a considerable modification in the shear stress distribution occur in heated supercritical flows. In Figure 2-19, total shear stress distribution is compared at two cross sections for case M2, one is at the inlet $(x / \delta=0)$ and the other is at $x / \delta=60.44$, further downstream near where the wall temperature peak is observed. As expected from the mean velocity distribution, the mean shear stress profile $\left(\bar{\tau} / \tau_{w, 0}\right)$ at $x / \delta=60.44$ differs from the inlet profile with the position of the zero mean shear stress moved closer to the heated inner

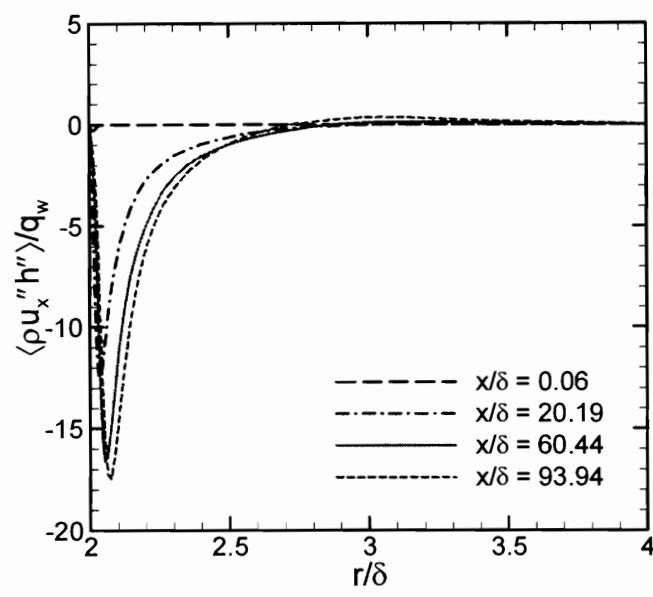

(a)

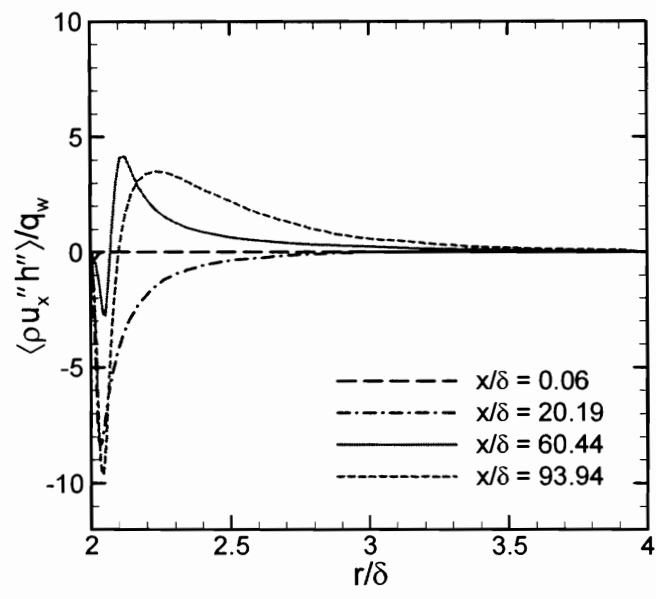

(b)

Fig. 2-20: Streamwise turbulent heat flux distributions, $\left\langle\rho u_{x}^{\prime \prime} h^{\prime \prime}\right\rangle / q_{w}$ : (a) case F2, forced convection; (b) case M2, mixed convection.

wall $(r / \delta=2.25)$. This position may be compared with that of the zero mean shear stress at the inlet where it is located at $r / \delta=2.89$, near the center of the channel. However, much more importantly, the Reynolds shear stress distribution $\left(-\overline{\rho u_{x}^{n} u_{r}^{n}} / \tau_{w, 0}\right)$ at $x / \delta=60.44$ shows a very large deviation from that of the inlet profile at $x / \delta=0$, indicating that the buoyancy effect causes a considerable modification in turbulence structure of the near wall region as in upward pipe flows.

In Figure 2-20, the radial profiles of the normalized streamwise turbulent heat flux $\left(\overline{\rho u_{x}^{\prime \prime} h^{\prime \prime}} / q_{w}\right)$ are compared between forced and mixed convections for cases F2 and M2, respectively. As shown in Figure 2-20(a), the streamwise turbulent heat flux in forced convection grows monotonically in the negative direction while it shows a non-monotonic behavior along the streamwise direction changing both signs and magnitudes in mixed convection with signficant buoyancy effect for case M2 (Figure 2-20(b)). As a consequence of this unconventional behavior of streamwise turbulent heat flux, mixed convection is subject to a non-monotonic temperature distribution as we have already examined in Figure 2-15.

These peculiar features of the thermal boundary layer of supercritical-pressure $\mathrm{CO}_{2}$ developing in a heated vertical annular channel are visualized in Figures 2-21 through 2-24. Two sections for case M3 at $Q^{+}=2.4$ are selected for visualization. The first section is in the range of $31.5 \leq x / \delta \leq 39.2$ in which the wall temperature approaches the maximum, and the second one is for $78.6 \leq x / \delta \leq 86.3$ in the downstream region where the wall temperature subsides substantially after the peak (see Figure 2-16). In Figure 2-21, three-dimensional features of thermal boundary layer are compared between the two sections, where the instantaneous iso-density surfaces are drawn at $T=T_{\mathrm{pc}}$. Some of the most identifiable features in these figures are bloated low density regions which are more spotty in the downstream region for $78.6 \leq x / \delta \leq 86.3$ than in the upstream region for $31.5 \leq x / \delta \leq 39.2$. As shown in Figures 2-22 and 2-23, these "bloated" low-density regions are actually the spots where an outward 
ejection of the hot fluid in the near wall region takes place. Because the turbulence level is reduced in the upstream region due to buoyancy effects, these ejection and sweeping motions of large scale turbulence are also reduced in the cross section so that thermal energy mixing due to the turbulent diffusion process occurs less vigorously than in the downstream region. This observation confirms the fact that in a heated boundary layer, the motion of the fluids in the thermal layer is closely associated with the motion of the large-scale turbulent structures since the heated fluids are transported and convected by turbulence. These large-scale turbulent structures were first observed by Kline et al. [1967], and since then became the active subject of investigation by many researchers. These studies have shown that turbulence production in the wall region is related to the motion of the large scale turbulence structures, and the qualitative information on the behavior of the fluid motion in the thermal boundary layer can be interpreted with reference to the evolution of these large-scale turbulence structures.

Figure 2-24 shows the instantaneous fluctuating velocity vectors in a transformed $X-Y$ plane superimposed on the instantaneous density contours in the downstream region for case M3. Of interest in this figure is that turbulent motions of the fluids near the pseudo-critical region (green colored region in the figure) are mostly directed to the streamwise direction, indicating that a positive streamwise turbulent heat flux $\left(\rho u_{x}^{\prime \prime} h^{\prime \prime}\right)$ is more likely to be realized if we are time-averaging these instantaneous fields. This fact is clearly shown in Fig. 2-20(b). Once again, this result is the most peculiar feature of supercritical-pressure fluids flowing upward in a vertical flow passage, which is proved to be the main cause of a severe impairment of turbulent heat transfer to supercriticalpressure fluids.

DNS development for complex non-circular geometries The third and final objective of the present task is to develop a DNS code to predict fluid flow and heat transfer characteristics for complex non-circular geometries. Although the work is in progress at the present time (aiming to simulate the ribbed annular flows with periodic spacers along the streamwise and circumferential directions [McCreery et al., 2003]), no presentable results are obtained yet. An immersed boundary method [Kim et al., 2001] is now successfully implemented in the code for annular flows with constant fluid property to model the grid spacers numerically. An initial code check has been completed for laminar flows at a fairly low Reynolds number in a ribbed annulus and more results are expected to evolve for turbulent flows at a higher Reynolds number.

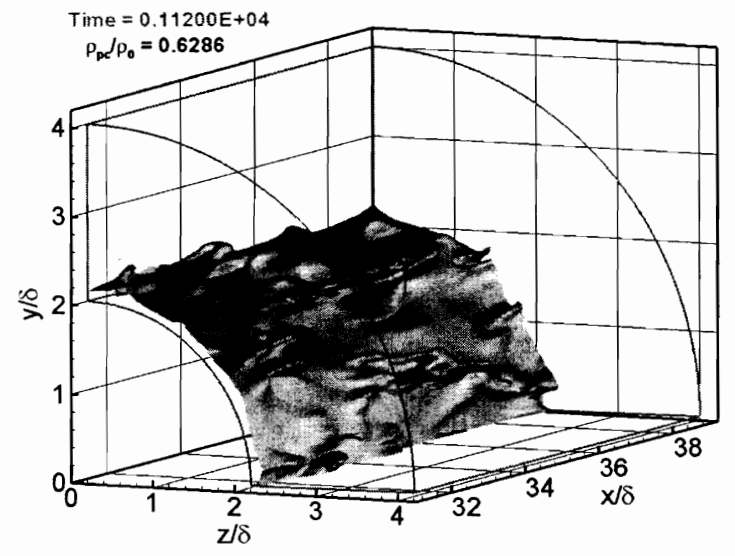

(a)

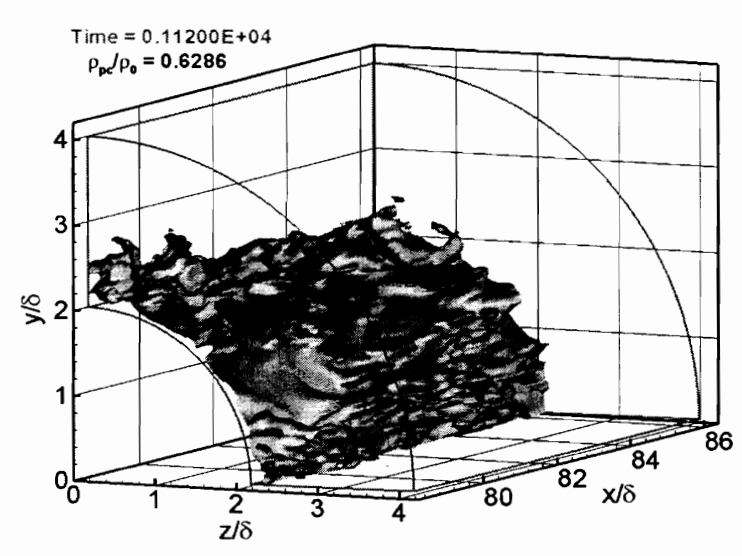

(b)

Fig. 2-21: Instantaneous iso-density surface at $T=T_{p c}$ (pseudo-critical temperature) for case M3, mixed convection: (a) $31.5 \leq x / \delta \leq 39.2$; (b) $78.6 \leq x / \delta \leq 86.3$. In this figure the original cylindrical coordinates of $(x$, $r, \theta)$ are transformed into the cartesian coordinates of $(x, y, z)$. 


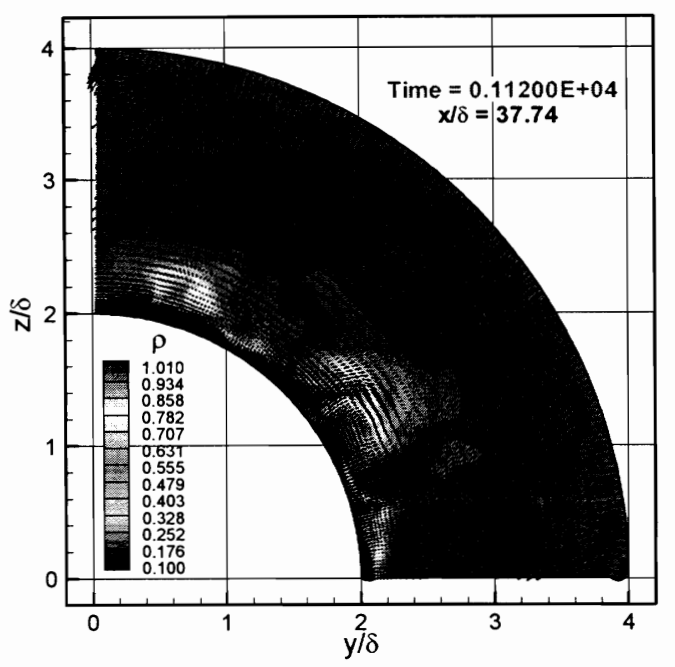

(a)

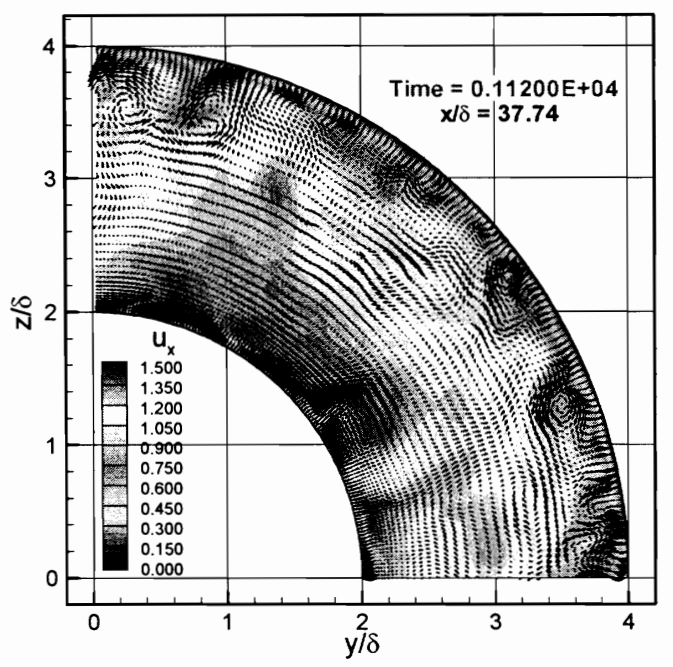

(b)

Fig. 2-22: Cross-stream velocity vector plots at $x / \delta=37.74$ superimposed on the instantaneous (a) density and (b) streamwise velocity contours for case $\mathrm{M} 3$, mixed convection.

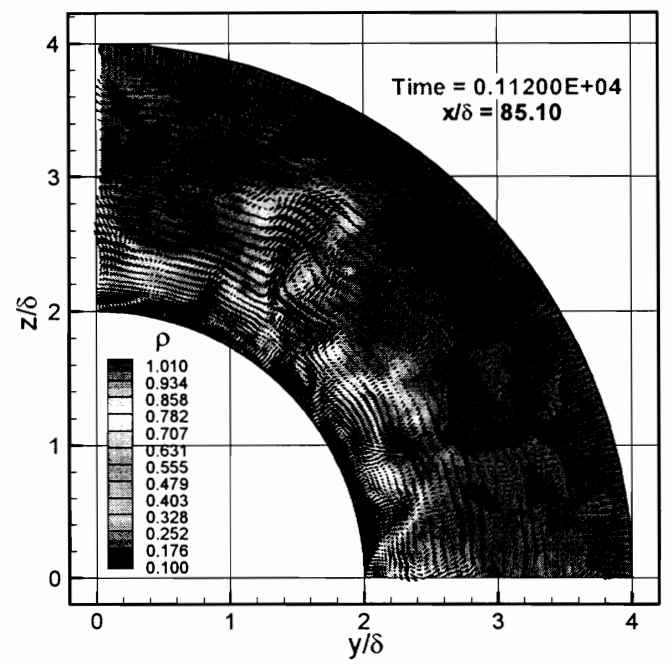

(a)

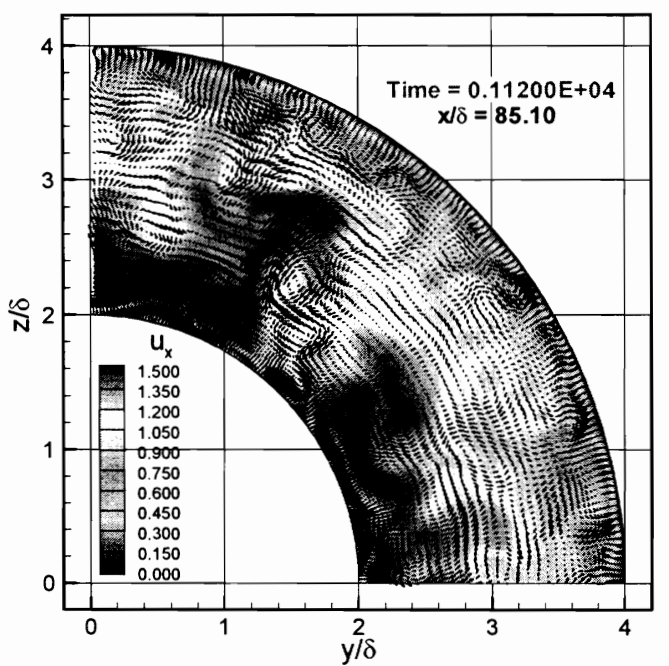

(b)

Fig. 2-23: Cross-stream velocity vector plots at $x / \delta=85.10$ superimposed on the instantaneous (a) density and (b) streamwise velocity contours for case $\mathrm{M} 3$, mixed convection. The vector size in this figure is reduced from that shown in Figure 4-22 for the purpose of clarity. 


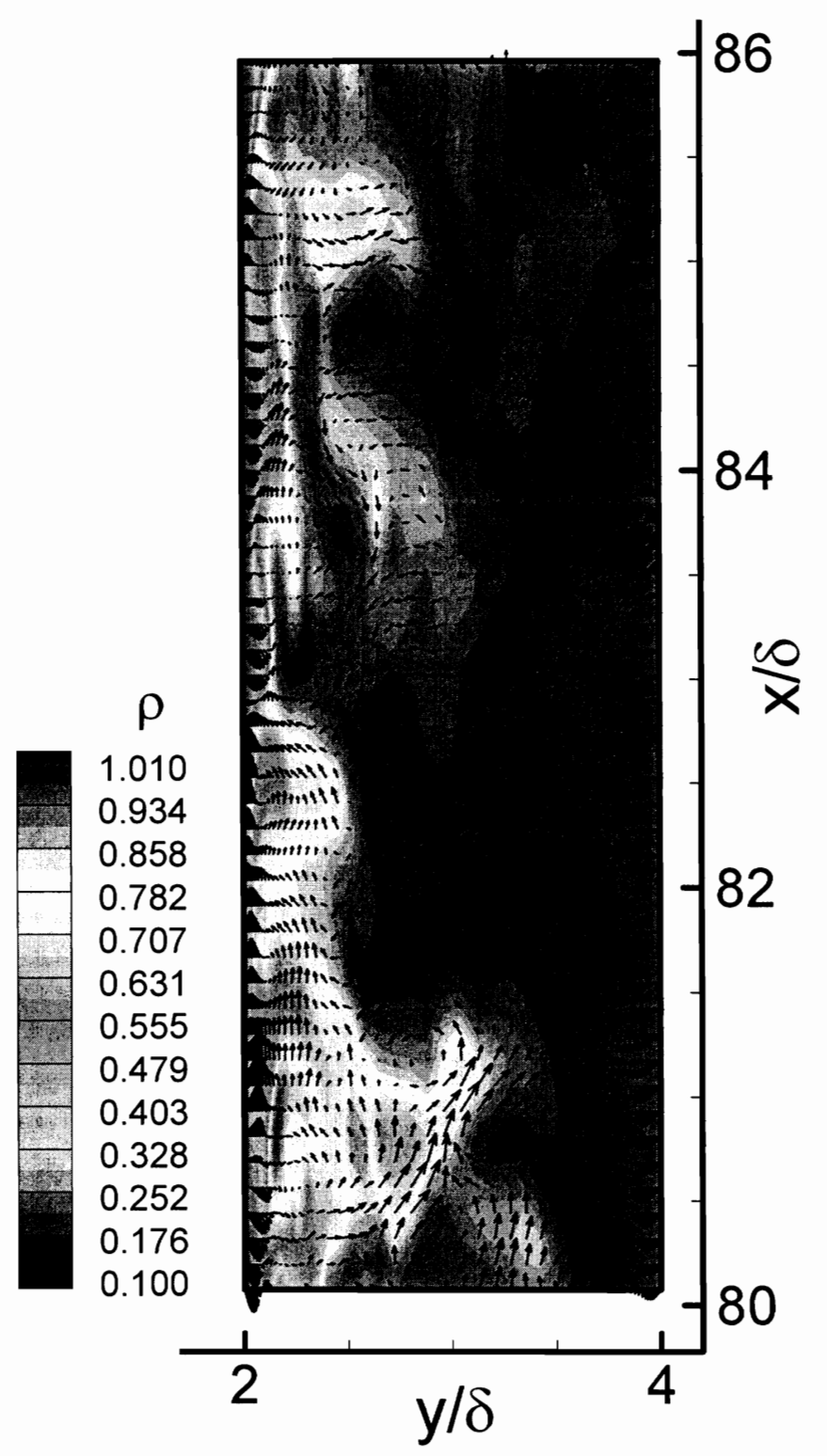

Fig. 2-24: Velocity fluctuation vectors in X-Y plane superimposed on the instantaneous density contours for case M3, mixed convection. 


\section{Task 3. Large eddy simulation (LES) development, Prof. R. H. Pletcher, lowa State University}

The main thrust of the research was to extend LES methodology to a broader class of flows with heat transfer, particularly flows characteristic of those occurring in ALWRs and SCRs. The objectives of this task were to extend the Iowa State LES capabilities to liquid, superheated and supercritical fluids with property variation and buoyancy in (a) circular tubes, (b) non-circular geometry as in tube banks and, ultimately, (c) idealized tube bank geometries with grid spacers.

The starting point for this research included computer codes for LES that had been validated by comparisons with experimental data for several flows in planar channels and pipes [Lee and Pletcher, 2001; Avancha and Pletcher, 2002; Dailey et al., 2003; Xu et al., 2004; Lee, Xu and Pletcher ,2004]. Lee and Pletcher [2001], Xu et al. [2004] and Lee, Xu and Pletcher [2004] focused on vertical heated flows in which the effects of buoyancy become important.

Prior to starting the present grant research, most of the simulations had been based on a quasi-developed computational model in which step-wise periodicity [Dailey, Meng and Pletcher, 2003] was assumed. Such an assumption appears to be reasonable for moderate heating rates. Figure 3-1 compares the computed temperature profile with measurements of Shehata and McEligot [1998] at $\mathrm{x} / \mathrm{D}=14.2$ while Figure 3-2 makes a similar comparison for $\mathrm{x} / \mathrm{D}=24.5$, the last station. This flow was heated moderately $\left(\mathrm{q}^{+} \approx 0.0018\right)$ and retained lowReynolds-number turbulent characteristics. Changes in simulated profiles from one streamwise location to the next can be clearly seen. The computational domain encompassed an axial distance of five diameters centered about the designated experimental location. The heat flux and Reynolds number from the experiment were set in the simulations and the wall temperature at the inlet of the computational domain was matched with the experimental data. Nusselt numbers agreed within two per cent. Further details on the simulations can be found in the paper by $\mathrm{Xu}$ et al. [2004]. The assumption of step-wise periodicity was used in the current project for isothermal flows and to generate inflow data for simulations in which heating levels required that flow development be taken into account.

The first research task was to modify and validate the formulation for the flow of liquids and superheated and supercritical fluids with variable properties in flow through a circular tube. Tables for the properties of carbon dioxide near the critical point were added to the code. The basic source of property data was PROPATH as in Task 2. An adiabatic wall simulation was completed for carbon dioxide entering a pipe at a pressure of $8 \mathrm{MPa}$ and a temperature of $295 \mathrm{~K}$. At this pressure, the pseudo-critical temperature, $T_{p c}$, is $307.85 \mathrm{~K}$. The inlet Reynolds number was 5400 . A $64 \times 40 \times 100$ grid (in the streamwise, radial and azimuthal directions, respectively) was used. The properties, especially the specific heat and density, vary significantly with small changes in temperature near the pseudo-critical point. These variations can be seen in Figures 3-3 and 3-4. Figure 3-5 shows the mean streamwise velocity profile from the LES for carbon dioxide near the critical point plotted in wall coordinates compared with constant property reference data (experimental results and a DNS simulation). For adiabatic flow the properties remain reasonably constant throughout the five-diameter length used in the simulation so the agreement with constant property data is fairly good. Figures 3-6 and 3-7 show similar comparisons for the rms velocities and shear stress, respectively. These results were obtained using the quasi-developed flow model that makes three assumptions: namely, (1) that the temperature variations in the streamwise direction are step-periodic for the uniform heat flux case, (2) that the mass flux is streamwise-periodic and (3) that the mean pressure variations across the flow are negligible in evaluating the density. This approach allows streamwise (and radial) property variations to be taken into account. The temperature, density, pressure and velocity can all vary in the streamwise direction. 


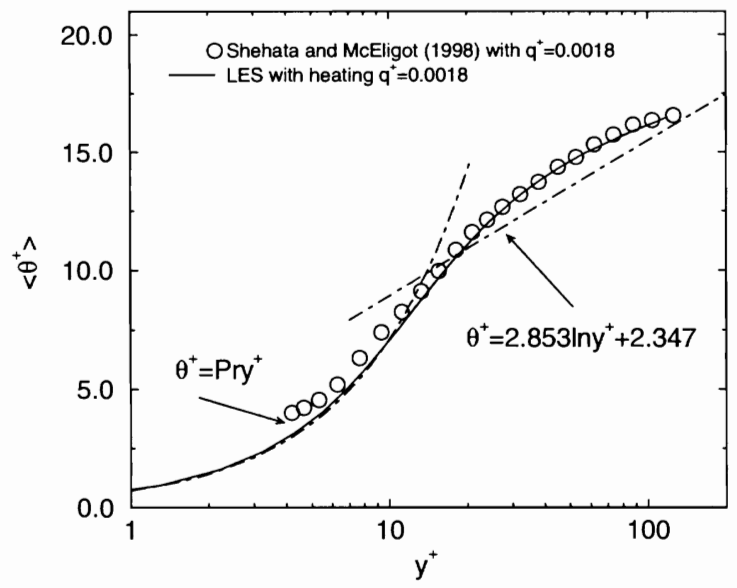

Fig. 3-1. Comparison of large eddy simulation with measurements of Shehata and McEligot [1998] at 14.2 diameters.

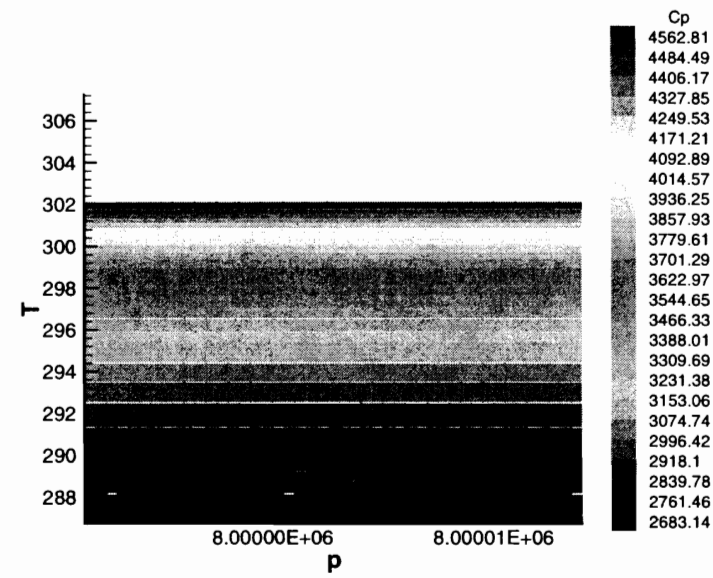

Fig. 3-3. Variation of specific heat of carbon dioxide with pressure and temperature. Pseudo-critical temperature, $\mathrm{Tpc}=307.85$ at $\mathrm{P}=8 \mathrm{MPa}$.

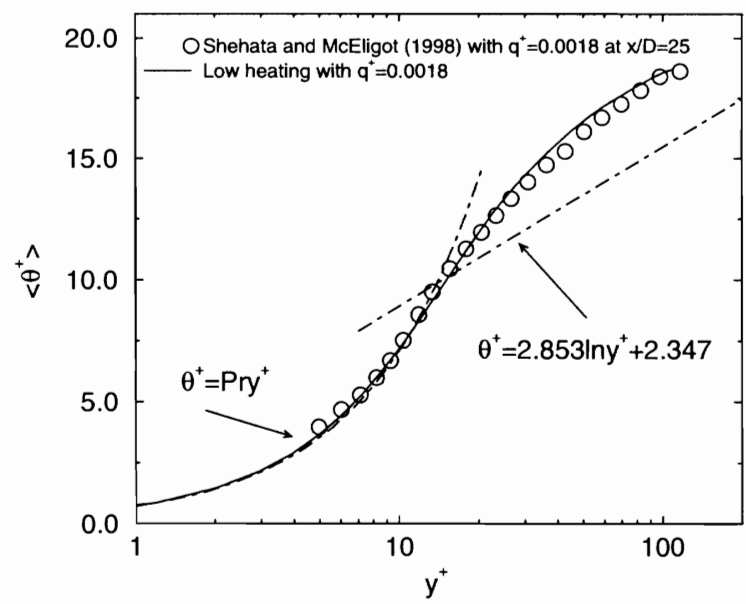

Fig. 3-2. Comparison of large eddy simulation with measurements of Shehata and McEligot [1998] at 24.5 diameters.

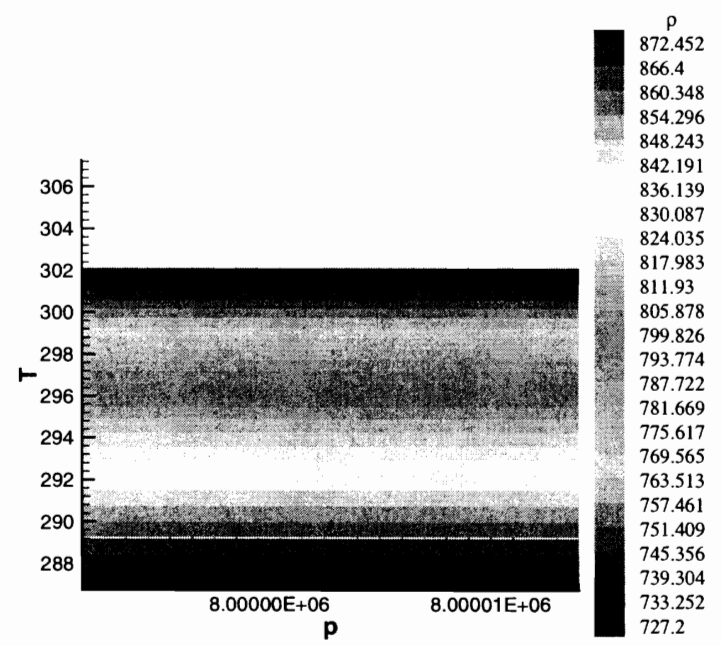

Fig. 3-4. Variation of density of carbon dioxide with pressure and temperature. Pseudo-critical temperature, $\mathrm{Tpc}=307.85 \mathrm{~K}$ at $\mathrm{P}=8 \mathrm{MPa}$. 


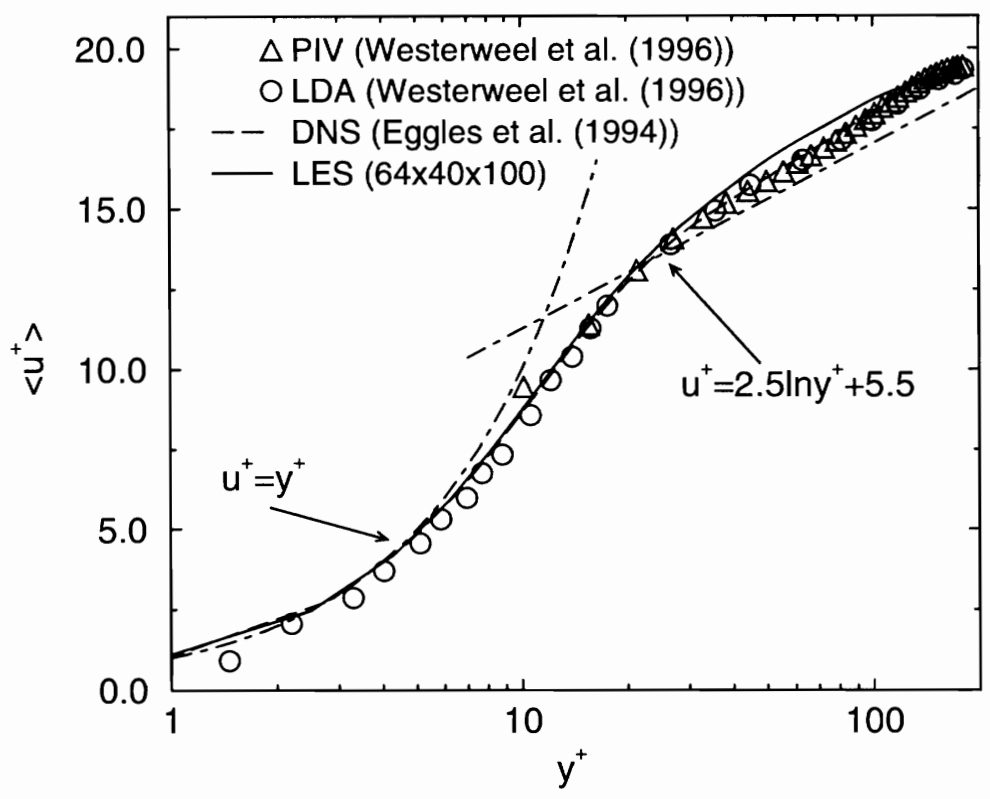

Fig. 3-5. Mean velocity profile compared to constant property experimental and DNS results for adiabatic flow of carbon dioxide near critical point in a circular tube, $\mathrm{Re}=5400$.

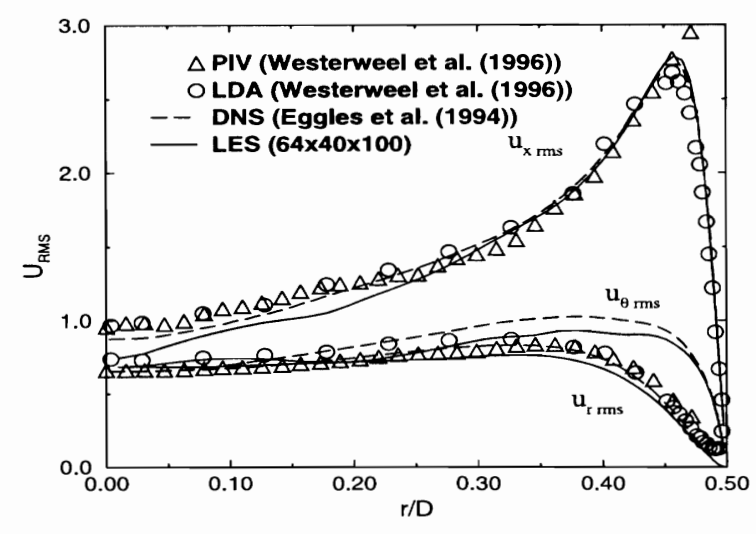

Fig. 3-6. Rms profiles (normalized with wall friction velocity) compared to constant property experimental and DNS results for adiabatic flow of carbon dioxide near critical point in a circular tube, $\mathrm{Re}=5400$.

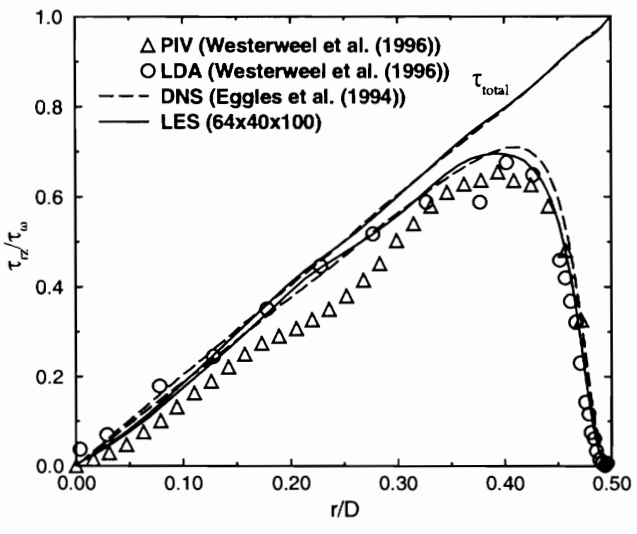

Fig. 3-7. Resolved and total stress compared to constant property experimental and DNS results for adiabatic flow of carbon dioxide near critical point in a circular tube, $\operatorname{Re}=5400$.

One important task was to extend the simulation capability to treat entrance region flows. This task was accomplished by implementing characteristic-based outflow conditions. Details are provided by Wang, Xu and Pletcher [2005]. This approach allowed the flow to develop within the computational domain. Inflow conditions to the region of primary interest (referred to as the "test section") were provided by adding an inflow generator region to the computational domain. The outlet flow of the inflow generator provided the inflow velocity and density conditions for the inlet to the test section. This approach fixes the mass flow rate. The test section inlet pressure, however, was extrapolated from the interior of the test section. The density and pressure were then used to fix the temperature at the inlet to the test section. The flow was isothermal in the inflow generator section and spatial stepperiodicity was used to create a hydrodynamically developed flow. The flow then typically underwent a degree of thermal development within the test section. 
Numerous comparisons of the large eddy simulation results with existing data and direct numerical simulations for liquid, superheated, and supercritical fluid flows were conducted. The simulation conditions are given in Table 3-1.

Table 3-1 Simulation Configurations for Large Eddy Simulations

\begin{tabular}{|c|c|c|c|c|c|c|c|}
\hline Case Name & SQNB & SQLTD1 & HQ3HT & AENB & AQNB & RANQ & HRHQ \\
\hline Working Fluid & $\mathrm{CO} 2$ & $\mathrm{CO} 2$ & $\mathrm{CO} 2$ & $\mathrm{CO} 2$ & $\mathrm{CO} 2$ & Air & $\mathrm{CO} 2$ \\
\hline $\begin{array}{l}\text { Inlet Pressure } \\
(\mathrm{P} 0, \mathrm{~Pa})\end{array}$ & $8.0 \mathrm{e} 6$ & $8.0 \mathrm{e} 6$ & $8.0 \mathrm{e} 6$ & $8.0 \mathrm{e} 6$ & $8.0 \mathrm{e} 6$ & ---- & $8.0 \mathrm{e} 6$ \\
\hline $\begin{array}{c}\text { Inlet Temperature } \\
(\mathrm{T} 0, \mathrm{~K}) \\
\end{array}$ & 295.15 & 295.15 & 301.15 & 301.15 & 301.15 & ----- & 288.15 \\
\hline $\begin{array}{c}\text { Reference Length } \\
\text { (L) }\end{array}$ & $\mathbf{R}$ (radius) & $\mathbf{R}$ (radius) & $\mathbf{R}$ (radius) & $\begin{array}{c}\mathbf{D}_{\mathrm{h}}=4 \delta \\
\text { (סhalf height) }\end{array}$ & $\begin{array}{c}D_{h}=4 \delta \\
\text { (סhalf height) }\end{array}$ & $\begin{array}{c}\mathbf{S}=2 \delta \\
\text { (spacing) }\end{array}$ & $\mathbf{R}$ (radius) \\
\hline $\begin{array}{c}\text { Reynolds Number } \\
\left(\text { ReL }=\mathrm{U} 0 \mathrm{~L} / \mathrm{V}_{0}\right)\end{array}$ & 2700 & 2700 & 2700 & 8900 & 8900 & 3450 & 14333 \\
\hline $\begin{array}{l}\text { Inlet Prandtl } \\
(\operatorname{Pr} 0)\end{array}$ & 2.48 & 2.48 & 3.08 & 3.08 & 3.08 & ----- & 2.24 \\
\hline Geometry & Pipe & Pipe & Pipe & Annulus & Annulus & $\begin{array}{l}\text { Ribbed } \\
\text { annulus }\end{array}$ & Pipe \\
\hline $\begin{array}{l}\text { Domain Size } \\
\text { (LxxLyxLz) }\end{array}$ & $10 \times 1.0 \times 6.28$ & $10 \times 1.0 \times 6.28$ & $10 \times 1.0 \times 6.28$ & $11.78 \times 1.0 \times 6.28$ & $14.0 \times 1.0 \times 1.57$ & \begin{tabular}{|c|}
$39.6 \times 1.0 \mathrm{x}$ \\
15.4 \\
\end{tabular} & $\begin{array}{c}14.0 \times 1.0 \mathrm{x} \\
6.28 \\
\end{array}$ \\
\hline $\begin{array}{l}\text { Grid Resolution } \\
\quad(\mathrm{ni}, \mathrm{nj}, \mathrm{nk})\end{array}$ & $64,40,100$ & $64,40,100$ & $64,50,100$ & $90,70,120$ & $70,50,50$ & $\begin{array}{l}70\left(55^{*}\right), 30, \\
36\left(15^{*}\right) \\
(* \text { rib } \\
\text { region) }\end{array}$ & $\begin{array}{c}70,130 \\
100\end{array}$ \\
\hline $\begin{array}{c}\text { HeatFlux } \\
\left(\mathrm{Q}^{+}=\mathrm{qwL} / \mathrm{k} 0 \mathrm{~T} 0\right)\end{array}$ & 0.25 & 0.25 & 2.4 & 1.2 & 1.2 & -.-- & 5.33 \\
\hline Flow Direction & Upward & Upward & Upward & Upward & Upward & ---- & Upward \\
\hline Flow Type & Forced & Mixed & Mixed & Forced & Forced & ---- & Mixed \\
\hline Other & ----- & ----- & ----- & $\begin{array}{c}\text { Entire domain is } \\
\text { computed } \\
\text { (inner wall } \\
\text { heated) }\end{array}$ & $\begin{array}{c}\text { Quarter domain } \\
\text { is computed } \\
\text { (inner wall } \\
\text { heated) }\end{array}$ & \begin{tabular}{|c|} 
Mesh \\
stretched in \\
$\mathbf{x}, \mathbf{y}, \mathbf{z}$ \\
direction. \\
\end{tabular} & ----- \\
\hline
\end{tabular}

(Note: the critical temperature and pressure of $\mathrm{CO} 2$ is $304.15 \mathrm{~K}$ and $7.4 \mathrm{Mpa}$, respectively.)

Validation comparisons were made with the DNS results at $\mathrm{x} / \mathrm{R}=3.05$ reported by our Korean colleagues for pipe flow of $\mathrm{CO}_{2}$ at two heat transfer rates. Figure 3-8 compares the mean streamwise velocity distribution obtained by LES with the DNS results for horizontal pipe flow at a Reynolds number of 5400 and with uniform heat flux heating of $\mathrm{Q}^{+}=0.25$. Figure 3-9 compares the mean velocity distributions in wall coordinates at the same location. The rms distributions for the pure forced convection case are compared in Figure 3-10. The wall and bulk temperature distributions are shown in Figure 3-11. It should be noted that the LES simulations employed a buffer zone of zero heat transfer near the outflow boundary which appears to influence the bulk temperature distribution. More recent simulations have shown that the use of a buffer zone is unnecessary but the simulations for this case have not been repeated because of demands on computational resources. Figure 3-12 shows the distribution of local Nusselt number for the forced convection case. When the flow was vertical and buoyancy forces (Jackson buoyancy parameter equal to $3.1446 \times 10^{-7}$ ) were taken into account, only small changes were expected because of the low heating level employed. However, the buoyancy and associated acceleration had a very noticeable effect on the mean velocity profile in wall coordinates at $x / R=3.05$ as can be seen in Figure 3-13. Figure 3-14 shows the distribution of rms fluctuations normalized with the friction velocity at $\mathrm{x} / \mathrm{R}=3.05$ for the same flow.

Comparisons with the Korean DNS data were also made for vertical pipe flow at the same Reynolds number but at a much higher heating rate of $\mathrm{Q}^{+}=2.4$. Figure 3-15 compares the mean streamwise velocity profiles at two 
locations, the pipe inlet and at $x / R=3.05$. Figure 3-16 compares the resolved Reynolds stress profiles at $x / R=3.05$ from the inlet to the test section. The agreement between the LES and DNS results is quite good. The turbulent kinetic energy normalized with the friction velocity at the inlet to the test section at $x / R=3.05$ is shown in Figure 317. The heating can be seen to reduce the level of kinetic energy. The instantaneous resolved stress distribution in the pipe can be seen in Figure 3-18. The temperature profiles in wall coordinates are compared in Figure 3-19.

Again, the LES and DNS results are in good agreement. The local Nusselt number distributions are shown in Figure 3-20. The LES results are slightly higher near the inlet but otherwise, the agreement is quite good.

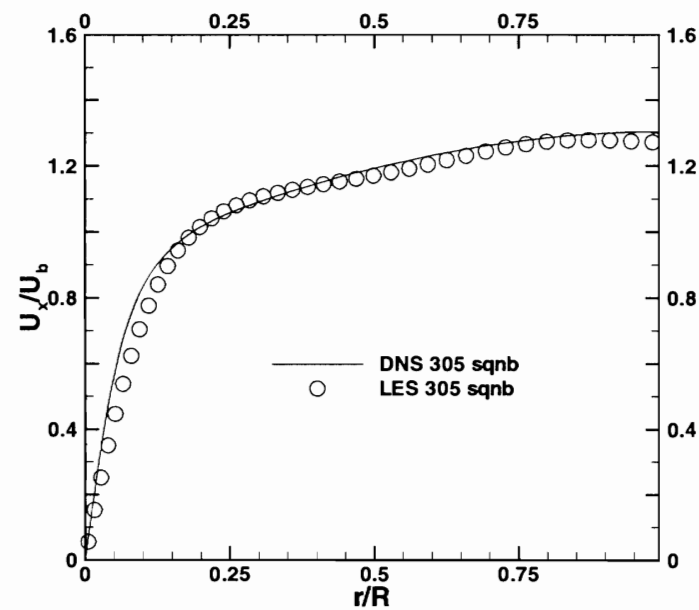

Fig. 3-8. Mean streamwise velocity distribution, $\mathrm{Re}=$ 5400 , forced convection.--- $x / R=3.05$, not considering the inflow generator section $(x / R=3.75)$.

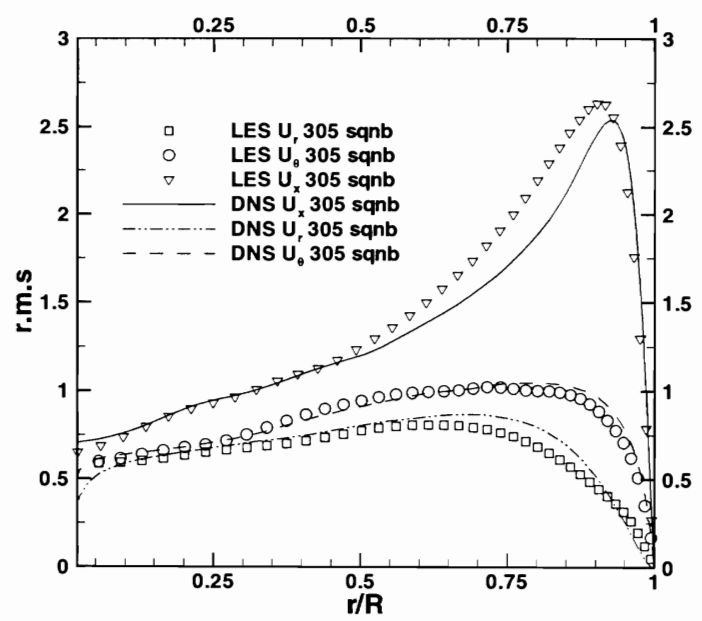

Fig. 3-10. Rms fluctuations normalized by wall friction velocity at $\mathrm{x} / \mathrm{R}=3.05$ from inlet to test section, forced convection.

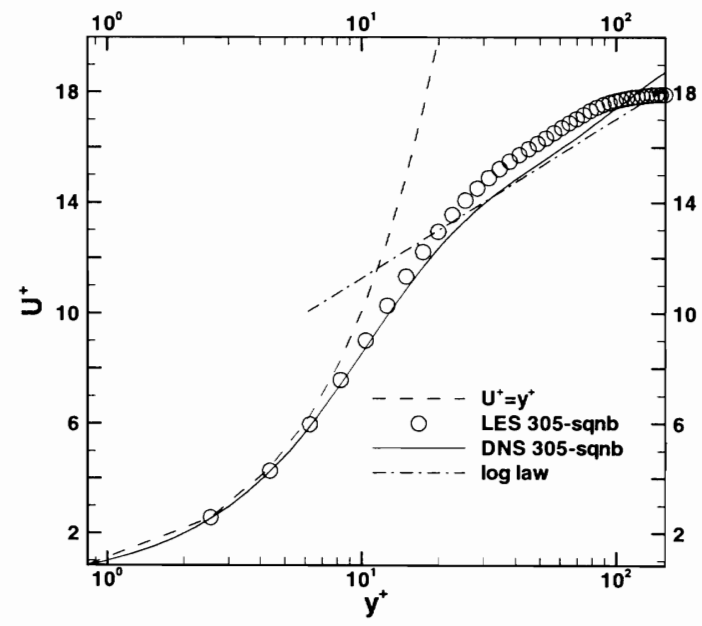

Fig. 3-9. Mean streamwise velocity distribution in wall coordinates at $\mathrm{x} / \mathrm{R}=3.05$ from inlet to heated test section; $\operatorname{Re}=5400$, forced convection.

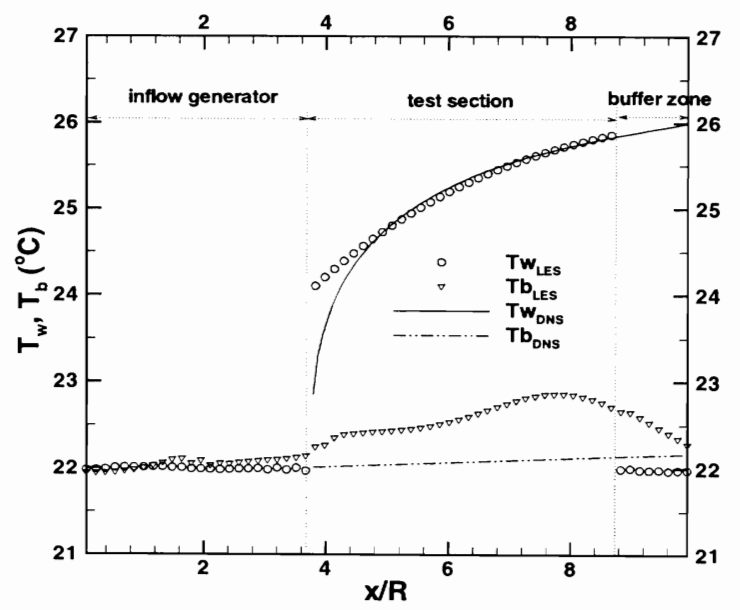

Fig. 11. Wall and bulk temperature distributions, forced convection case; 


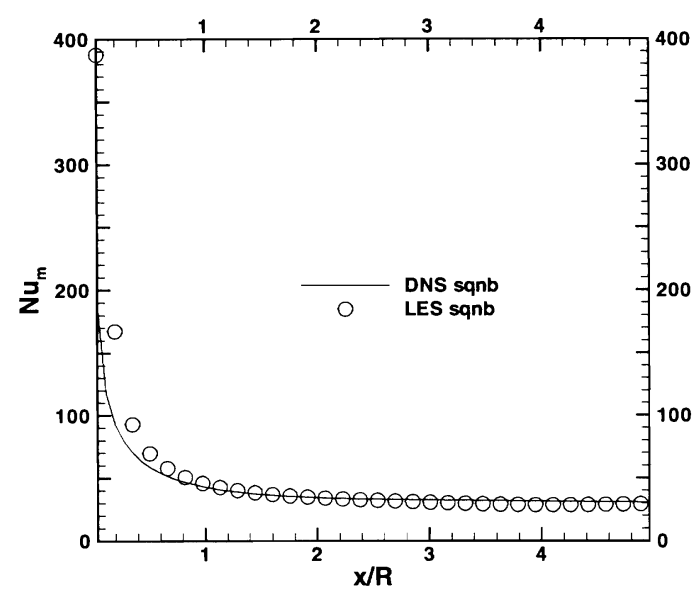

Fig. 3-12. Distribution of local Nusselt number, forced convection case (inflow generator and buffer zone are not included).

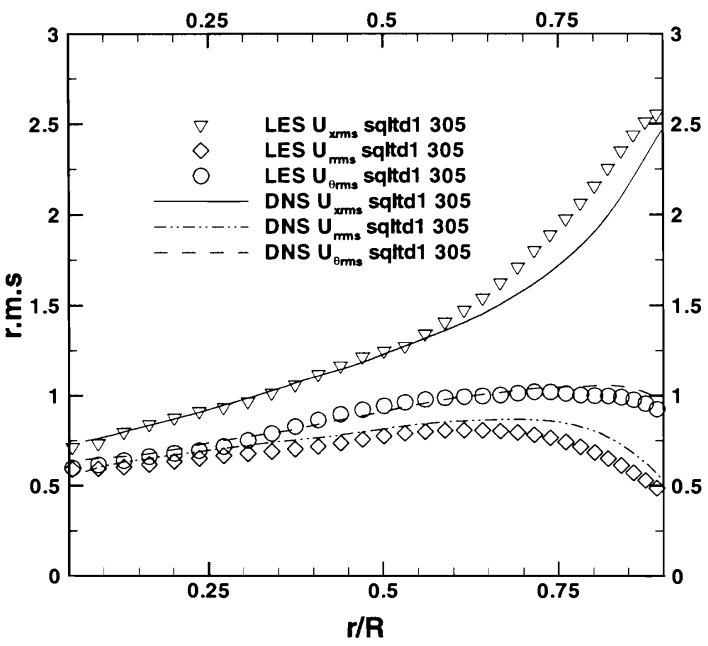

Fig. 3-14. Rms fluctuations normalized by wall friction velocity, mixed convection; ( u'rms $=\left(<u^{\prime} 2>\right) 1 / 2$, where $\diamond$ denotes an average in the streamwise and circumferential directions and in time.)

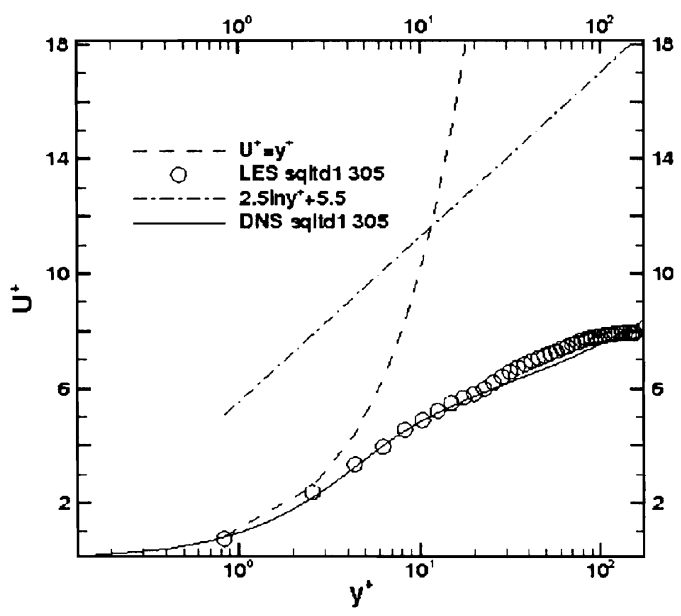

Fig. 3-13. Mean streamwise velocity distribution in wall coordinates, $\operatorname{Re}=5400$, mixed convection, Jackson parameter $=3.1446 \times 10^{-7}$.

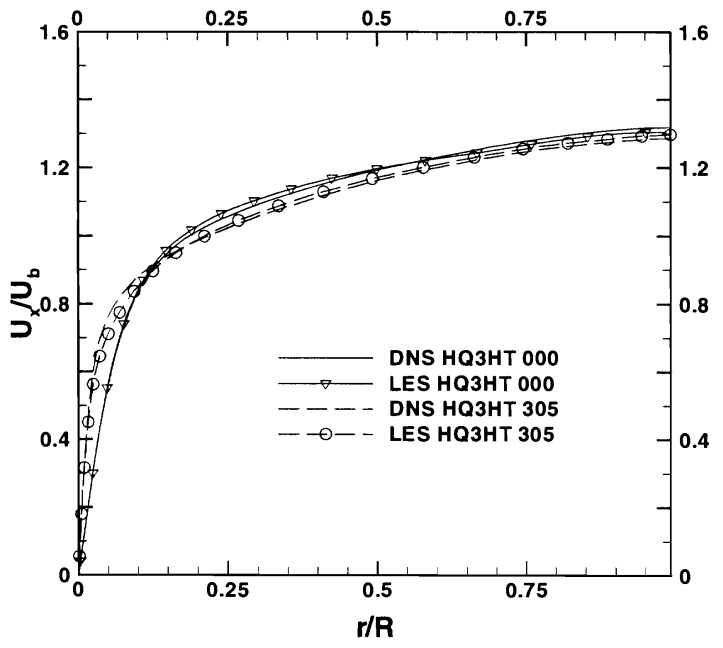

Fig. 3-15. Mean streamwise velocity distribution, $R e=$ 5400 , mixed convection, high heating. 

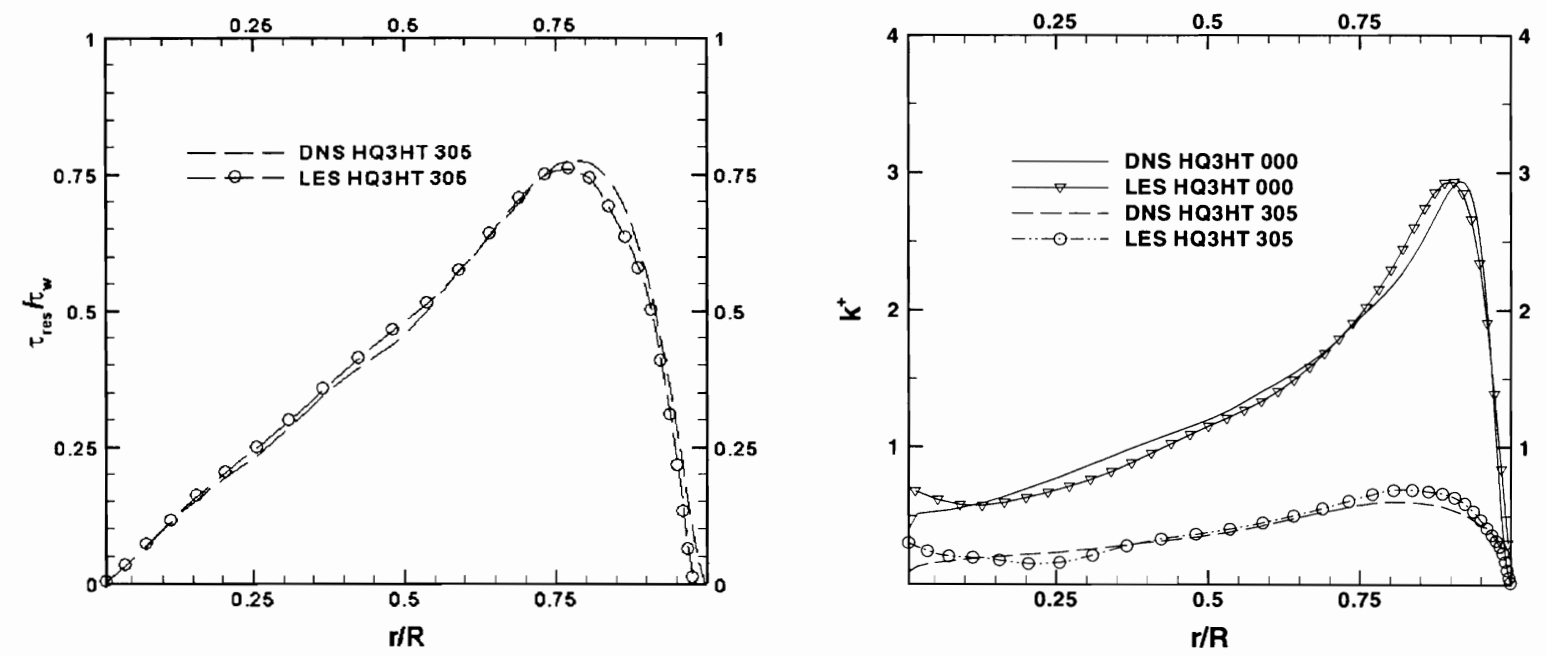

Fig. 3-16. Mean resolved Reynolds stress distribution at $\mathrm{x} / \mathrm{R}=3.05$ from inlet to test section, $\mathrm{Re}=5400$, mixed convection, high heating.

Fig. 3-17. Turbulent kinetic energy distribution at two locations, $\operatorname{Re}=5400$, mixed convection, high heating.

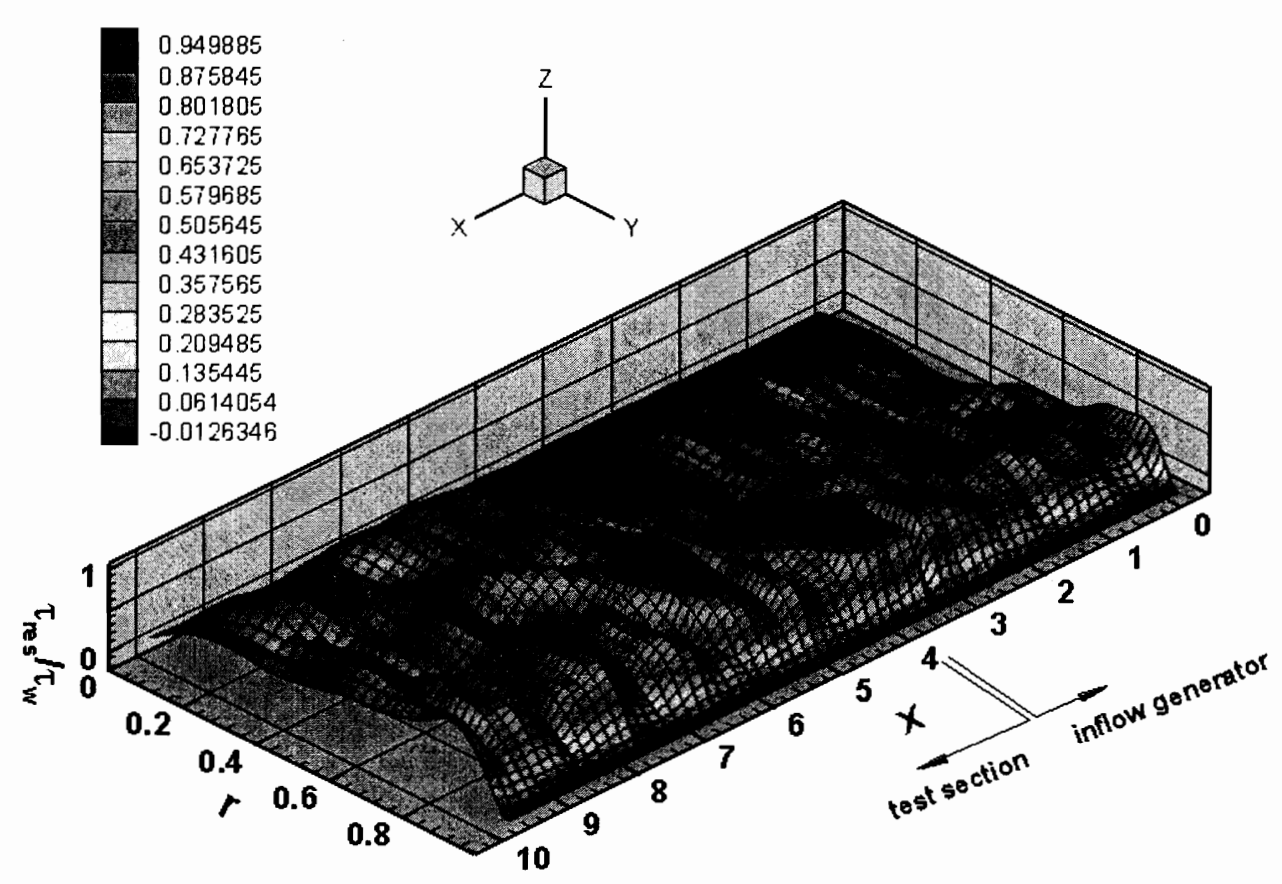

Fig. 3-18. Instantaneous resolved Reynolds stress, $\mathrm{Re}=5400$, mixed convection, high heating. 


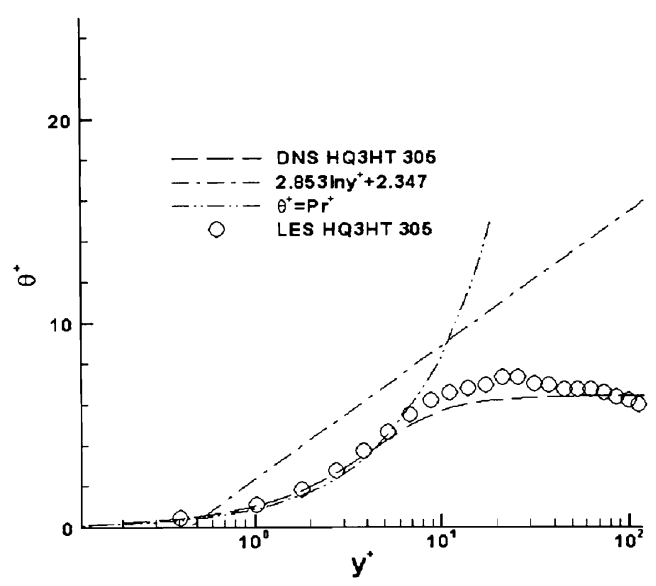

Fig. 3-19. Temperature distribution in wall coordinates at $\mathrm{x} / \mathrm{R}=3.05$ from inlet, $\mathrm{Re}=5400$, mixed convection, high heating.

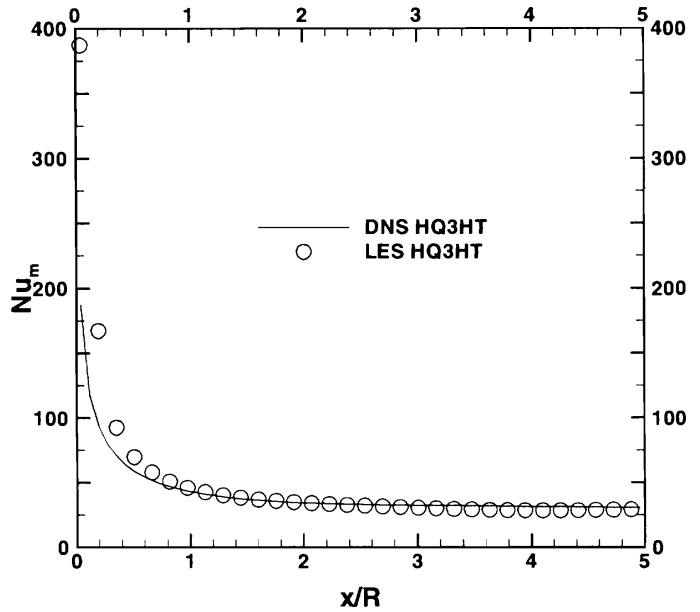

Fig. 3-20. Nusselt number distribution, $\mathrm{Re}=5400$, mixed convection, high heating.

Another goal has been to simulate the supercritical fluid flow in a vertical annular passage. The configuration for the simulation is shown in Figure 3-21. Fully-developed isothermal flow at a Reynolds number of 8900 was used as the inlet condition for the heat transfer cases. Figure 3-22 compares the distribution of the mean velocity normalized with the friction velocity at the inlet obtained by LES and DNS [Bae et al., 2005]. Figure 3-23 shows the same data plotted on wall coordinates from the outer wall. The streamwise rms fluctuations are compared with DNS results reported by Chung et al. [2002] for the same Reynolds number in Figure 3-24. Example results for annular flows at $\mathrm{Re}=8900$ have also been obtained for the forced convection case (case F2 of Bae et al., [2005]) with uniform heat flux heating at $\mathrm{Q}^{+}=1.2$ on the inner wall. Figure 3-25 compares the LES and DNS mean streamwise velocity results at $x / D_{h}=3.77$ in wall units. The agreement is quite good. Figure 3-26 shows a similar comparison of the computed temperature distribution in wall units. The agreement is reasonably good, but both the LES and DNS results deviate greatly from constant property results. The rms results are compared in Figure 3-27. Some differences can be observed, especially in the $u_{\mathrm{rms}}$ distribution. Contours of the instantaneous streamwise component of velocity are shown in Figure 3-28. The magnitude of the velocity variations appears to diminish with distance along the flow, presumably due to the influence of heating on the structure of turbulence. Figure 3-29 compares the predictions of Nusselt number. 

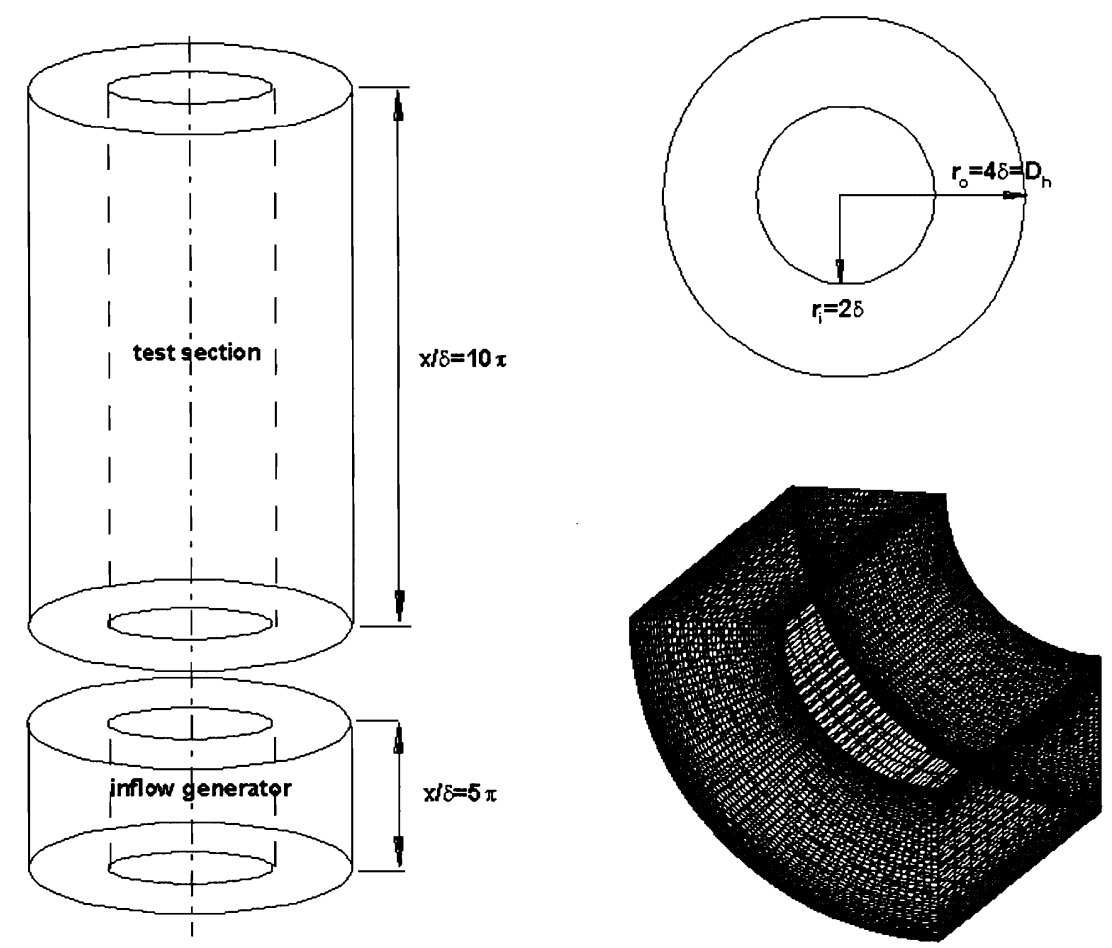

Fig. 3-21. Computational domain for LES of vertical annular passage.

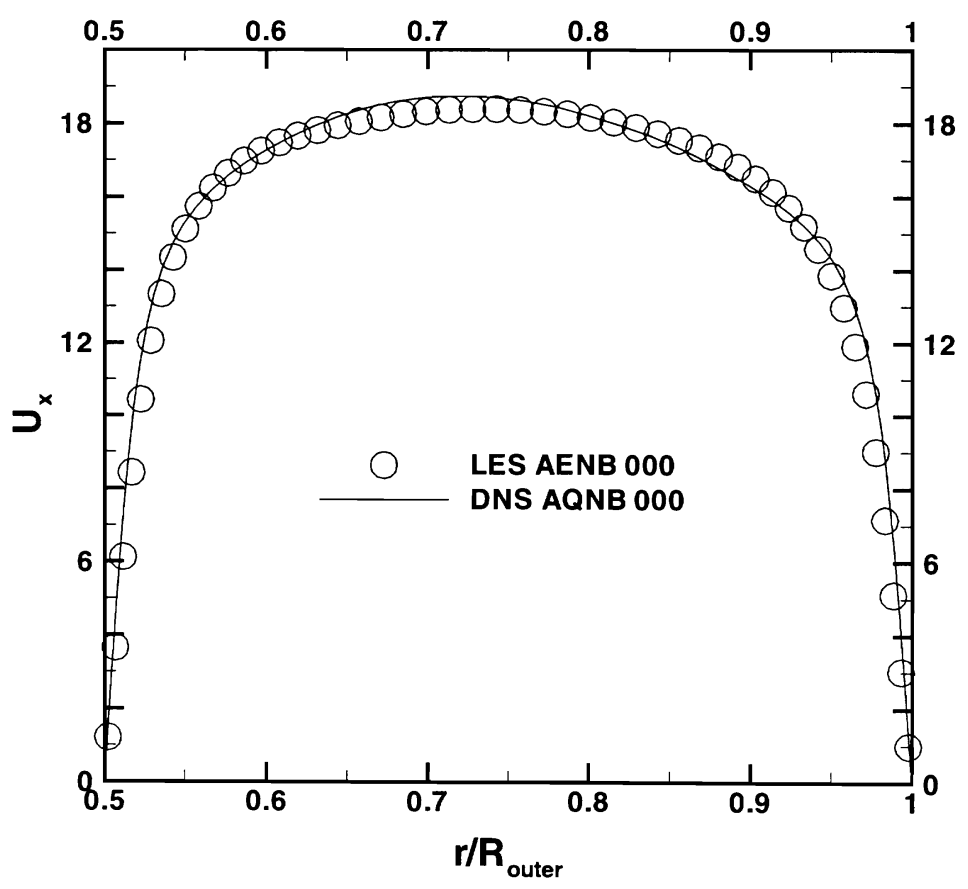

Fig. 3-22. Mean velocity distribution at $x / R=0$, vertical annular flow, $r_{i} / r_{o}=0.5, R e=8,900$. 


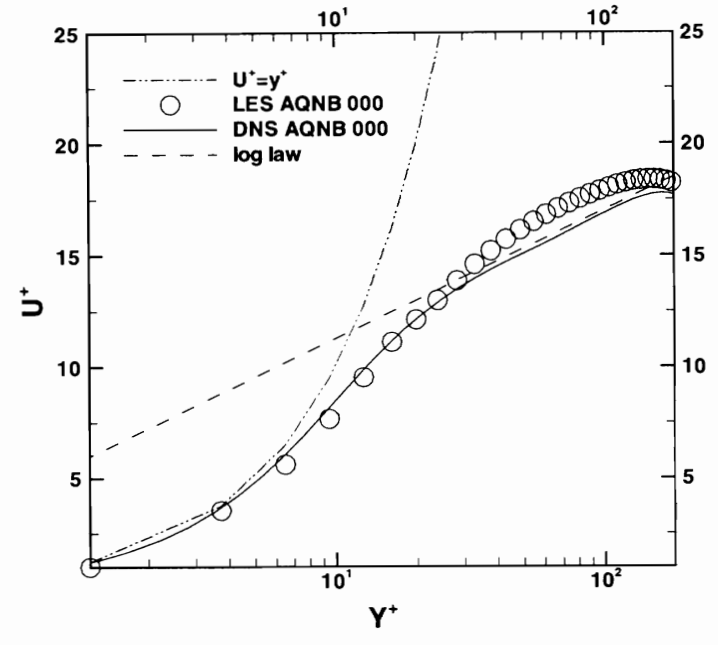

Fig. 3-23. Mean velocity distribution in outer wall coordinates at $\mathrm{x} / \mathrm{R}=0$, vertical annular flow, $\mathrm{r}_{\mathrm{i}} / \mathrm{r}_{\mathrm{o}}=0.5$, $\operatorname{Re}=8,900$.

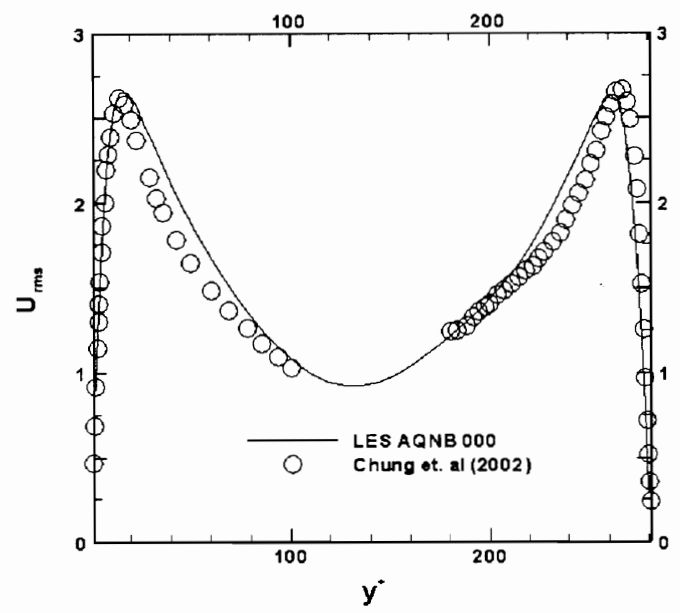

Fig. 3-25. Streamwise rms fluctuations at inlet to test section in inner wall coordinates normalized by inner wall friction velocity, vertical annular flow, $r_{i} / r_{0}=0.5$, $\operatorname{Re}=8,900$.

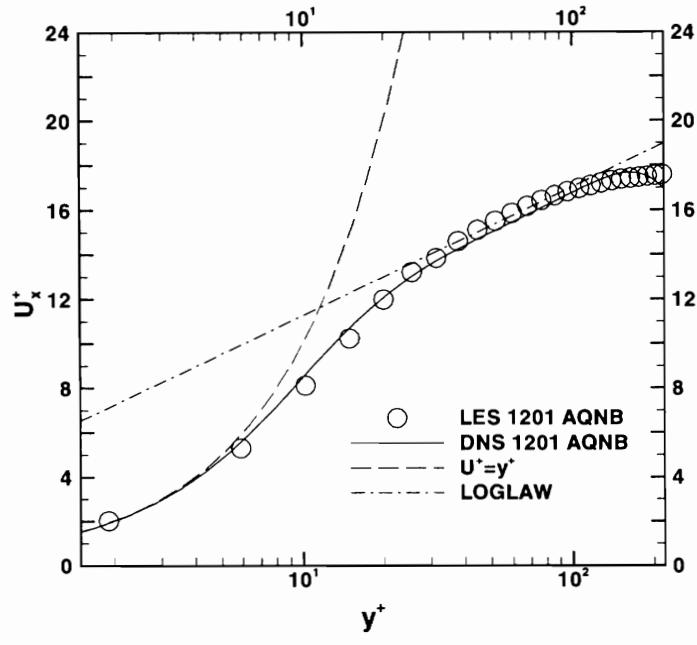

Fig. 3-24. Mean velocity distribution in outer wall units, annular flow, $\mathrm{r}_{\mathrm{i}} / \mathrm{r}_{\mathrm{o}}=0.5, \operatorname{Re}=8,900, \mathrm{Q}^{+}=1.2, \mathrm{x} / \mathrm{D}_{\mathrm{h}}=$ 3.77 .

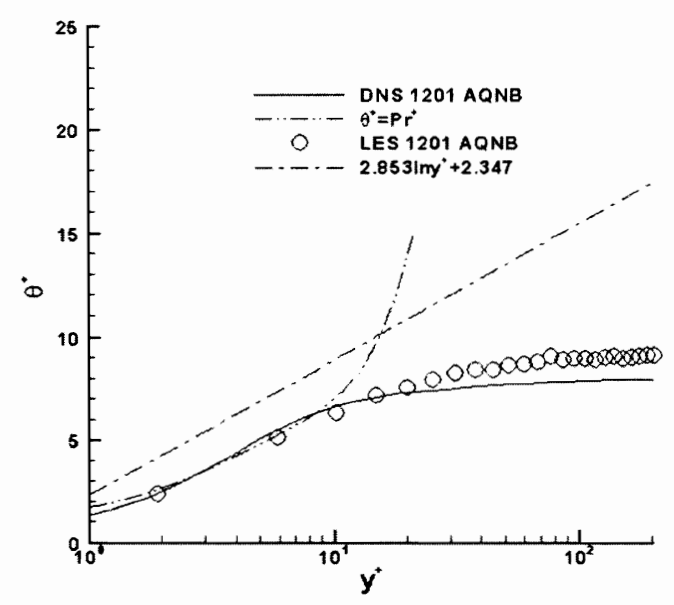

Fig. 3-26. Mean temperature distribution in wall units, annular flow, $\mathrm{r}_{\mathrm{i}} / \mathrm{r}_{\mathrm{o}}=0.5, \operatorname{Re}=8,900, \mathrm{Q}^{+}=1.2, \mathrm{x} / \mathrm{D}_{\mathrm{h}}=$ $3.77\left(1201\right.$ indicates $\left.x / D_{h}=1.201 \pi\right)$. 


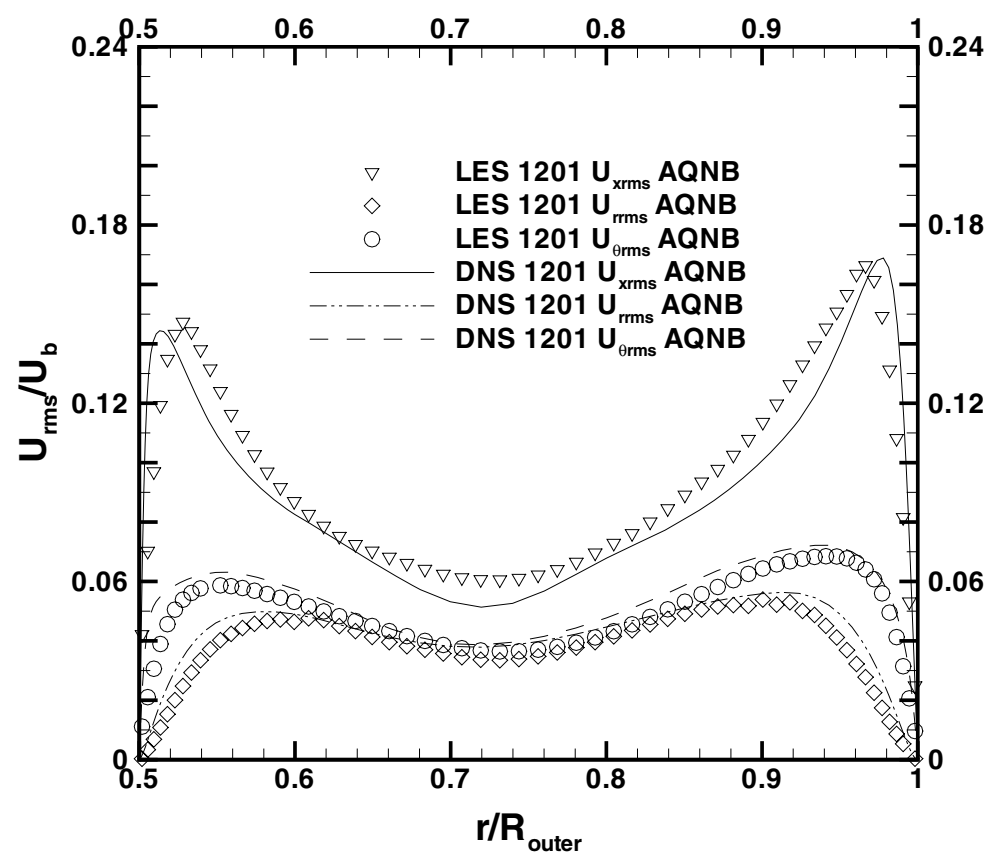

Fig. 3-27. Rms fluctuations normalized with bulk velocity, annular flow, $\mathrm{r}_{\mathrm{i}} / \mathrm{r}_{\mathrm{o}}=0.5, \mathrm{Re}=8,900, \mathrm{Q}^{+}=1.2, \mathrm{x} / \mathrm{D}_{\mathrm{h}}=$ 3.77 .

$\mathbf{x} / \delta=10.31$
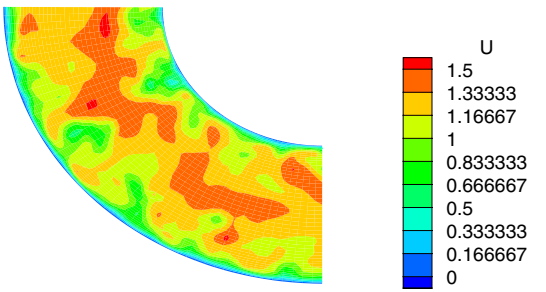

$x / \delta=20.125$

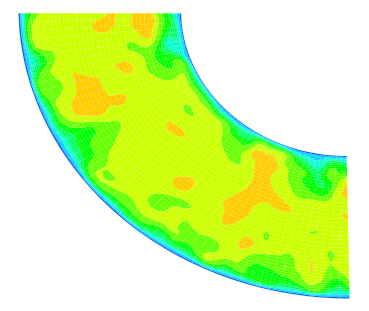

$\mathbf{x} / \delta=15.09$

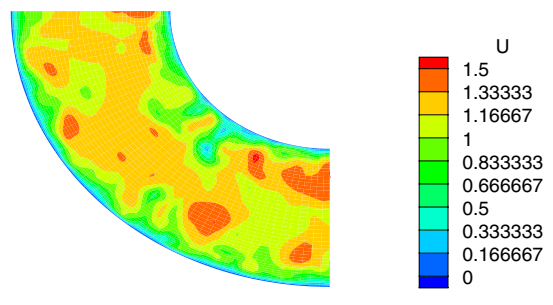

$\mathrm{x} / \delta=\mathbf{3 0 . 1 8 9}$

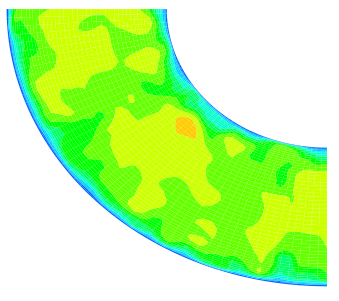

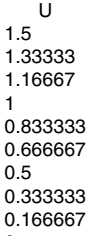

Fig. 3-28. Streamwise instantaneous velocity contours, annular flow, ri/ro $=0.5, \operatorname{Re}=8,900, \mathrm{Q}^{+}=1.2 ; \delta$ is half the distance between walls where $\mathrm{U}$ is normalized by $\mathrm{U} 0$, the inlet bulk velocity. 


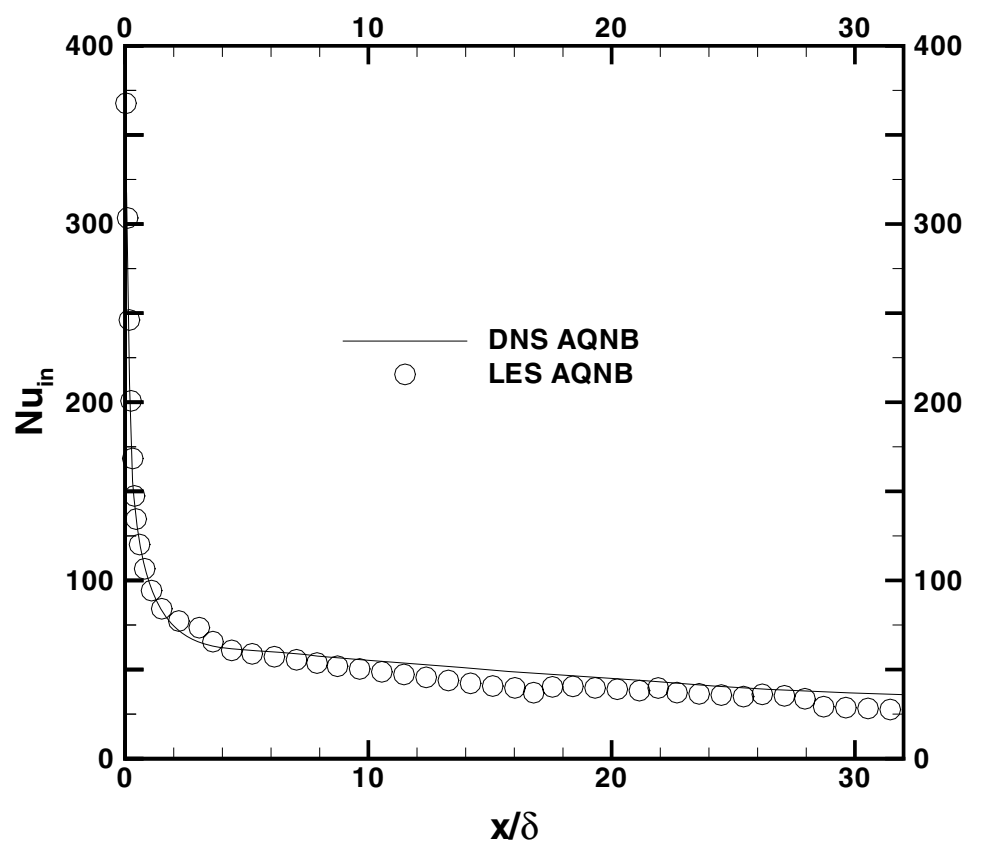

Fig. 3-29. Nusselt number (inner wall) distribution, annular flow, $\mathrm{r}_{\mathrm{i}} / \mathrm{r}_{\mathrm{o}}=0.5, \mathrm{Re}=8,900, \mathrm{Q}^{+}=1.2 ; \delta$ is half the distance between walls.

Simulations are currently underway for pipe flow at $\mathbf{R e}=\mathbf{2 8 , 6 6 6}$ with wall heating at a level $\mathrm{Q}^{+}=5.33$ corresponding to a case studied experimentally by Prof. Kim's group at SNU (Task 7), where $\mathrm{D}_{\mathrm{h}}=7.8 \mathrm{~mm}, \mathrm{q}=40$ $\mathrm{kw} / \mathrm{m}^{2}$ and mass flow rate $=0.015 \mathrm{~kg} / \mathrm{s}$. Some instantaneous temperature and streamwise velocity contours are shown in Fig. 3-30. A $70 \times 130 \times 100$ grid in the streamwise, wall normal and azimuthal directions, respectively, was used after several checks to determine the effects of grid refinement.

Another part of the final task was to extend the LES capability to more complex geometries. Two procedures have been used. For the ribbed annulus case studied experimentally by McCreery et al. [2003], the LES code for annular passages was modified to provide no-slip boundary conditions for the spacer ribs. The rib shapes were readily fit by the grid employed for the annular geometry. For the more complex shapes studied experimentally at INL consisting of rods contained within a rectangular enclosure (Task 5), the immersed boundary technique [Fadlun et al., 2000] was used. The immersed boundary technique is a general procedure that, in principle, can be used to represent any three-dimensional configuration.

For the ribbed annulus case, spatial periodicity was enforced in the simulations. The Reynolds number based on hydraulic diameter and bulk velocity was 6,900. The experimental and computational streamwise arrangements are shown in Figures 3-31, 3-33 and 3-34. As for the experimental apparatus, the spacer sets consisted of four ribs so their circumferential pitch was ninety degrees. However, only one of them was taken into account in our study, which is shown in Figure 3-31. According to the measurements by McCreery et al. [2003], the flow was considered streamwise-periodic. 

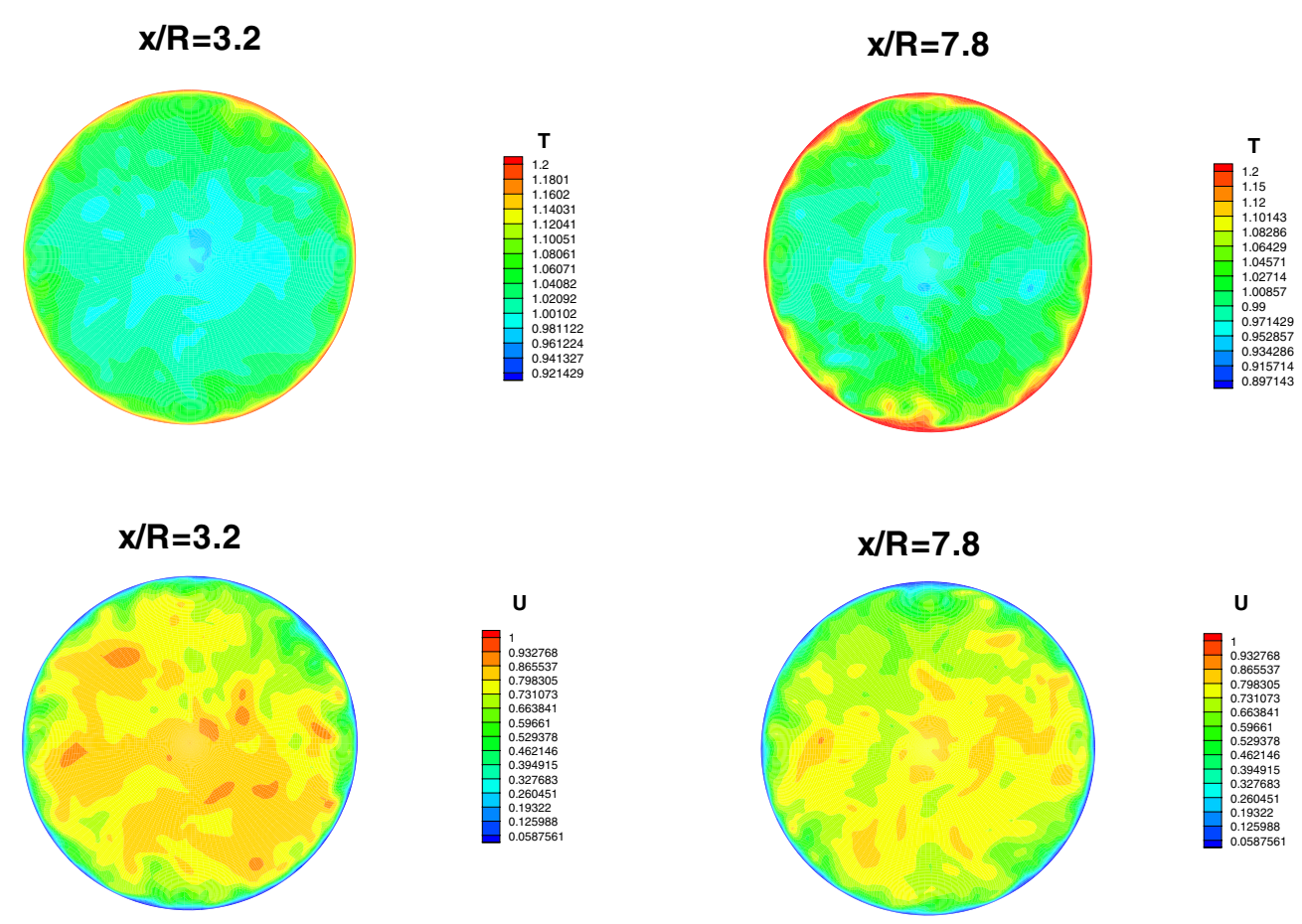

Fig. 3-30. Sample instantaneous streamwise velocity contours, pipe flow, $R e=28,666, Q^{+}=5.33$, where $T$ and $U$ are normalized by the bulk temperature and velocity, $\mathrm{T}_{0}$ and $\mathrm{U}_{0}$, respectively ( $\mathrm{T}_{\mathrm{pc}}$ corresponds to approximately 1.056 in the temperature contours).

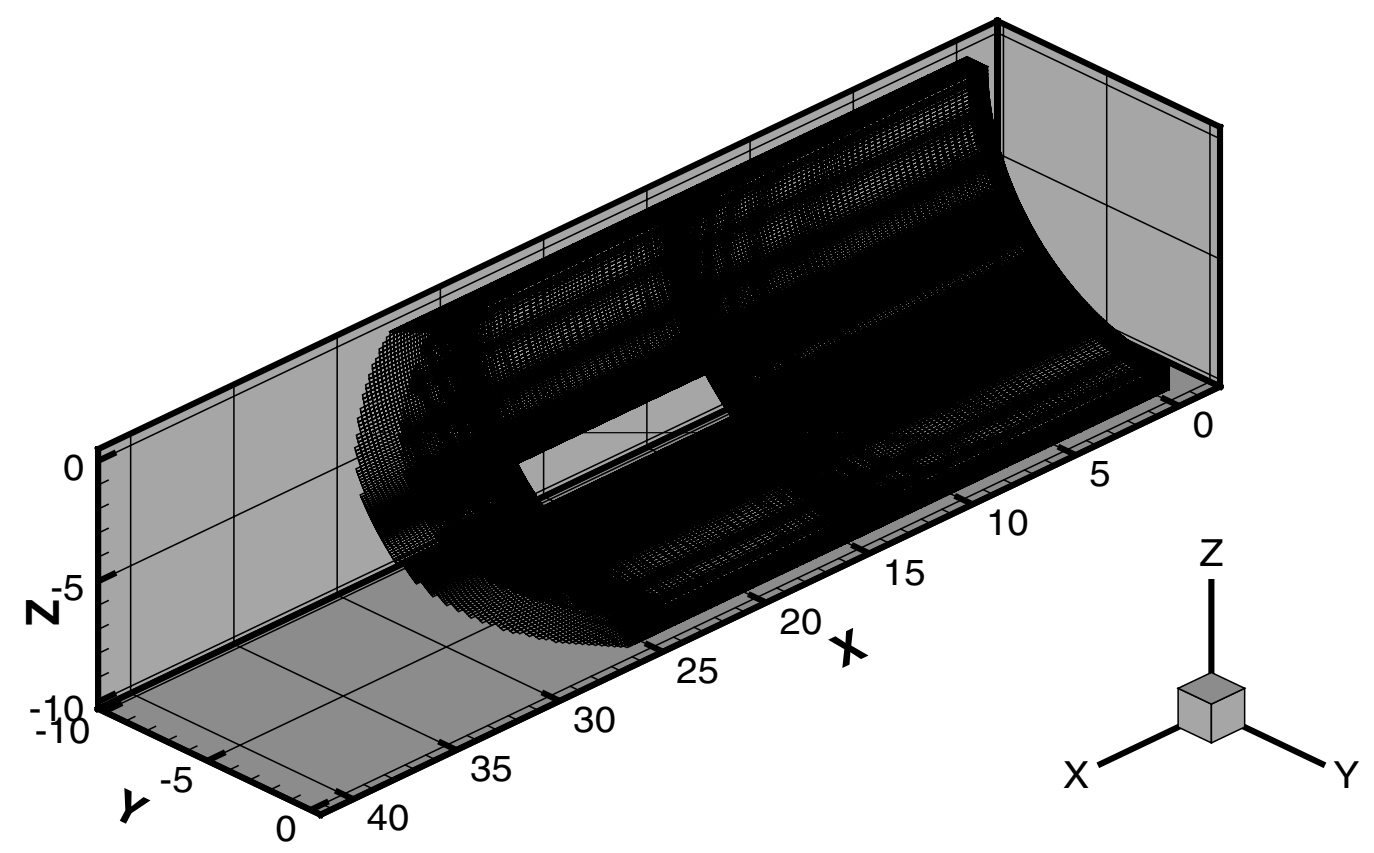

Fig. 3-31. Computational domain for LES, ribbed annulus. 
To keep the mass flow through the computational domain constant with time and to satisfy the periodic assumption, a time and $\mathrm{x}$-location dependent forcing term was introduced, representing the mean pressure gradient in the streamwise direction. The average streamwise pressure gradient was calculated dynamically at each physical time step to provide the desired mass flow rate. The initial estimation and evolution equations were developed through the governing equations of a two-dimensional, incompressible channel flow [Dang and Morchoisne, 1989]. Figure 3-32 shows the mass flow rate variation with time at two different $x$-locations, which are the downstream and upstream of the rib region. The variation of $U$ along an axis midway between the walls $(r)$ and midway between the ribs $(\theta)$ is presented in Figure 3-35.

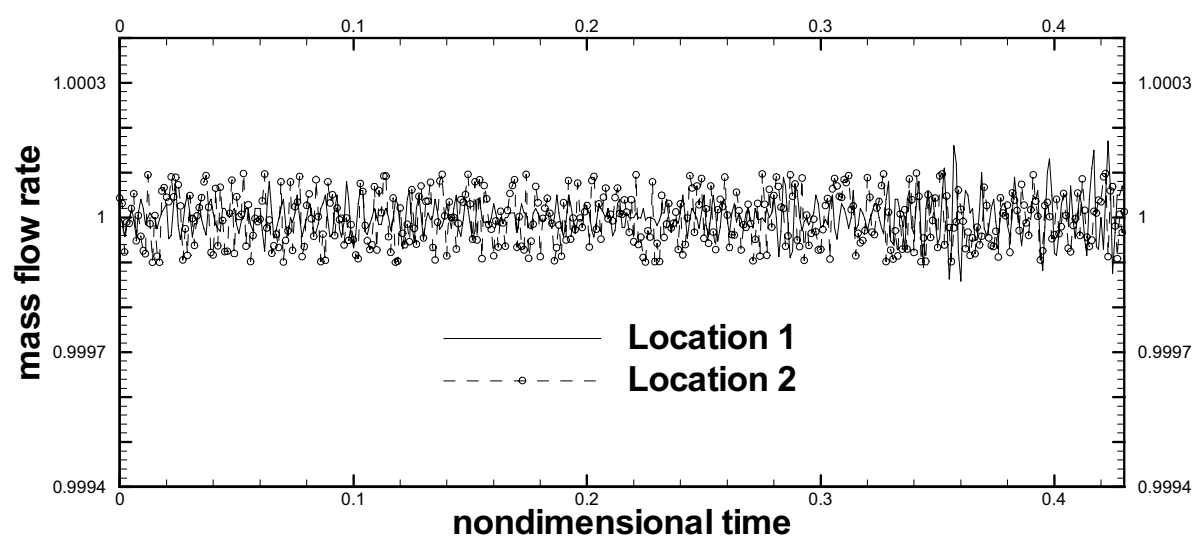

Fig. 3-32. Mass flow rate variation with time and $x$-location.

Comparisons between LES simulations and the experimental results of McCreery et al. [2003] for three radial traverses of mean streamwise velocity, U, (U-764, U-703, U-362) taken along the circumferential centerplane are provided in Figures 3-36 to 3-38. They are located sequentially near the point of maximum deceleration (764), near the midpoint axially (362) and near the point of maximum acceleration (703). In Figures 3-36 to 3-38 one should note that the experimental data may need some adjustment since the wall spacing is nominally $7.7 \mathrm{~mm}$ but some of the experimental profiles indicate a larger spacing; in other words, the maximum value of (r-rinner ) appears to be $8.0 \mathrm{~mm}$ rather than $7.7 \mathrm{~mm}$. This difference is especially noticeable in Figures 3-36 and 3-38. The simulation results in Figure 3-39 suggest that a finer grid may be desirable near the wall since the laminar sublayer is not as accurately resolved as it should be. On the other hand, pressure gradients are present in this flow which may cause some departure from the "law"-of-the-wall.

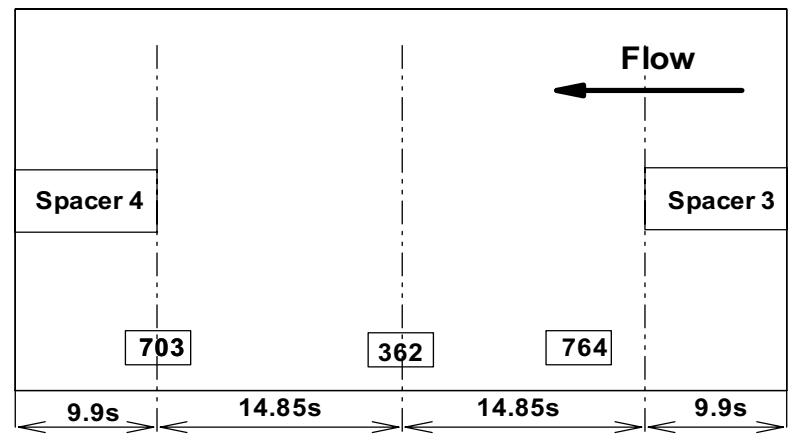

Fig. 3-33. Experimental arrangement.

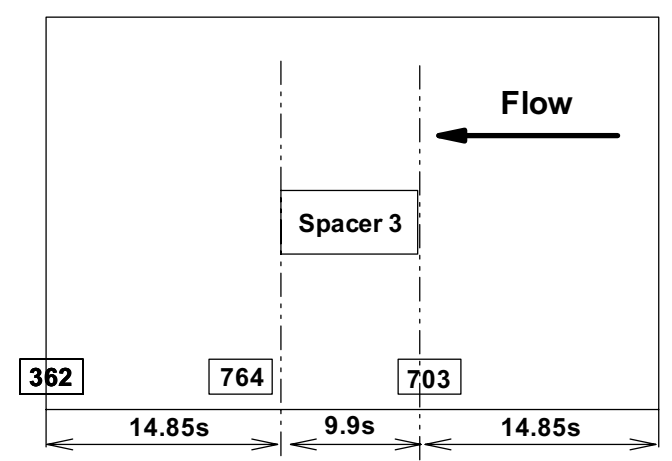

Fig. 3-34. Computational domain with periodicity. 


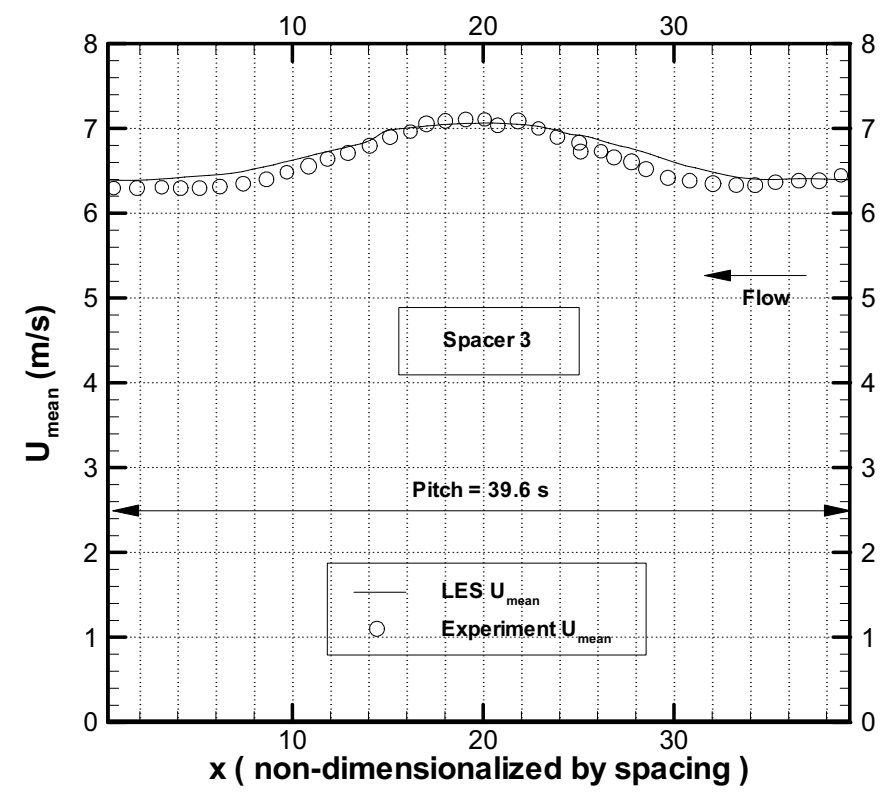

Fig. 3-35. Axial distribution of mean streamwise velocity halfway between ribs at mean radius, $R e=6900$

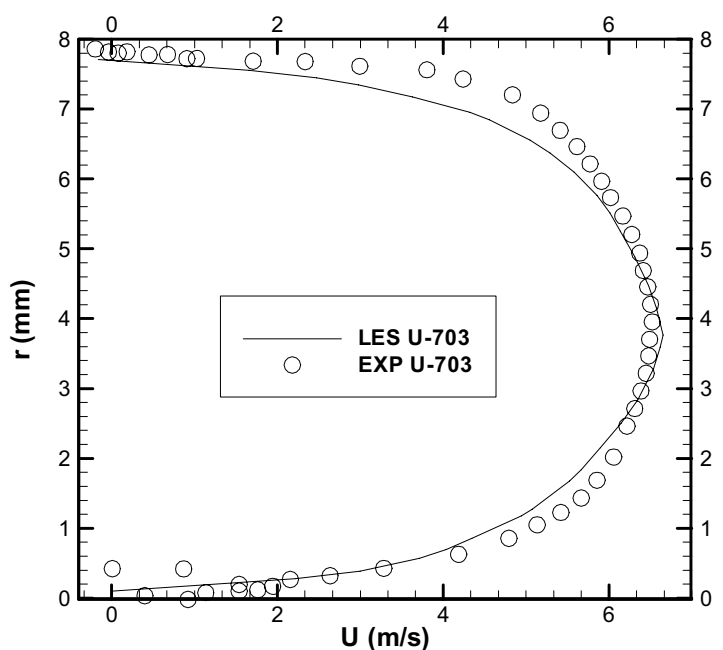

Fig. 3-36. Radial profile of mean streamwise velocity along centerplane at $\mathrm{x}$ location 703 .

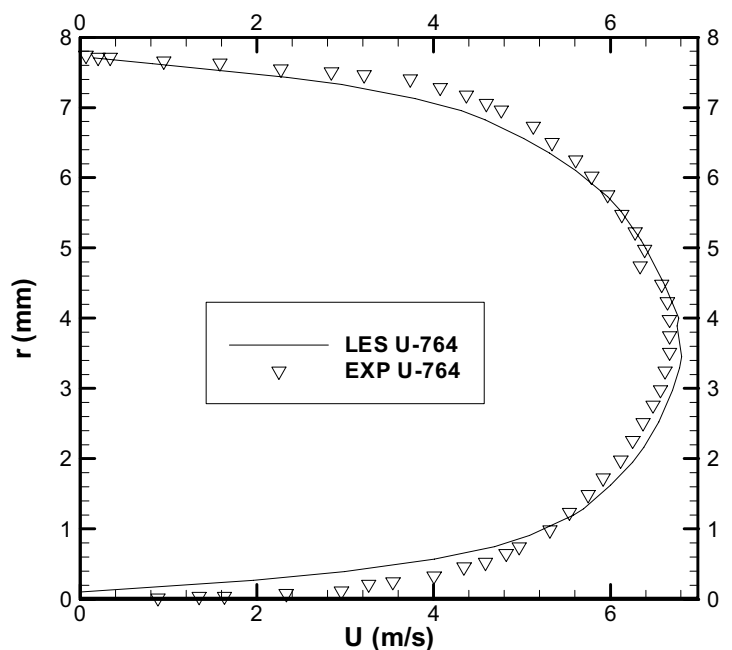

Fig. 3-37. Radial profile of mean streamwise velocity along centerplane at $\mathrm{x}$ location 764 . 


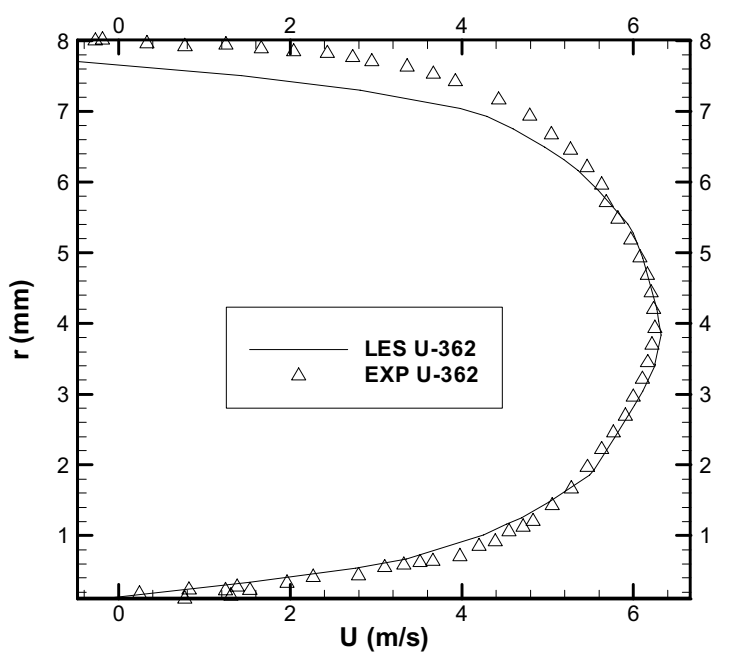

Fig. 3-38. Radial profile of mean streamwise velocity along centerplane at $\mathrm{x}$ location 362 .

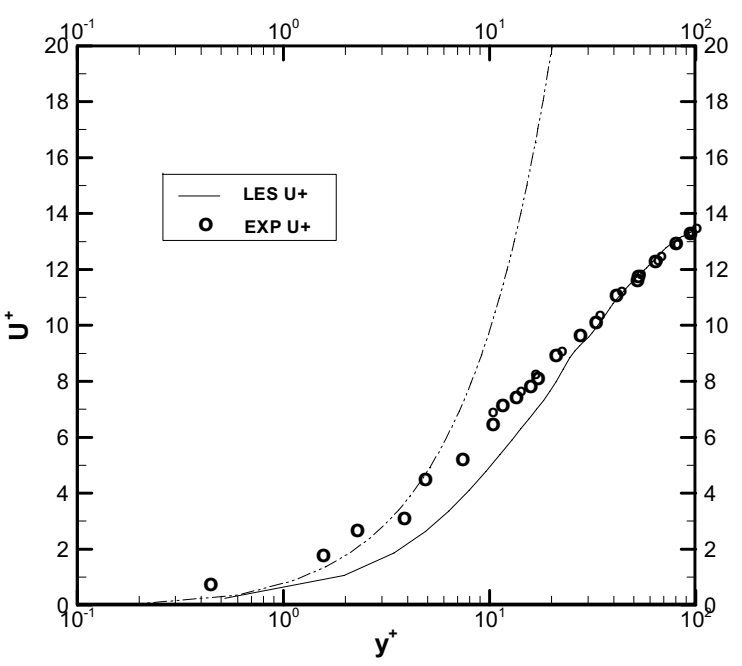

Fig. 3-39. Inner wall behavior at location 362 (see Figure 3-33).

The immersed boundary method for treating complex geometries is currently under development as a part of this project. Most of the simulation models for turbulent flow capable of handling geometrical complexity have been schemes using generalized body-fitted curvilinear meshes or unstructured grid meshes. Historically, these were developed within the framework of the Reynolds-averaged Navier-Stokes (RANS) approach. When these methods are applied to LES or DNS applications, they are often found to be too dissipative [Morinishi et al., 1998].

As an alternative to boundary-conforming strategies, non-body fitted grid methods have also been developed. These are mainly classified as Cartesian grid methods or immersed boundary methods. With these, a high level of accuracy can be maintained within the computational domain, but it is challenging to represent boundary conditions as accurately as can be done with boundary-conforming grid methods.

In the immersed boundary method, the boundary conditions on any boundary that does not conform to the grid lines of the simulation are normally met by adding computational points outside the flow boundary and adding a forcing function to the equations near the boundary points in such a way that the solution in the neighborhood of the boundary approximately meets the required boundary conditions. The procedures used by various investigators have varied in detail. Since grid points will not generally lie exactly on the boundary, an interpolation scheme must be employed to deduce the conditions on the boundary [Balaras, 2004].

The immersed boundary method was tested first for some laminar flows in relatively simple geometries. Two such test cases were laminar flow in an annulus and pipe flow using a Cartesian-based grid. The grid layout for the pipe case is shown in Figure 3-40.

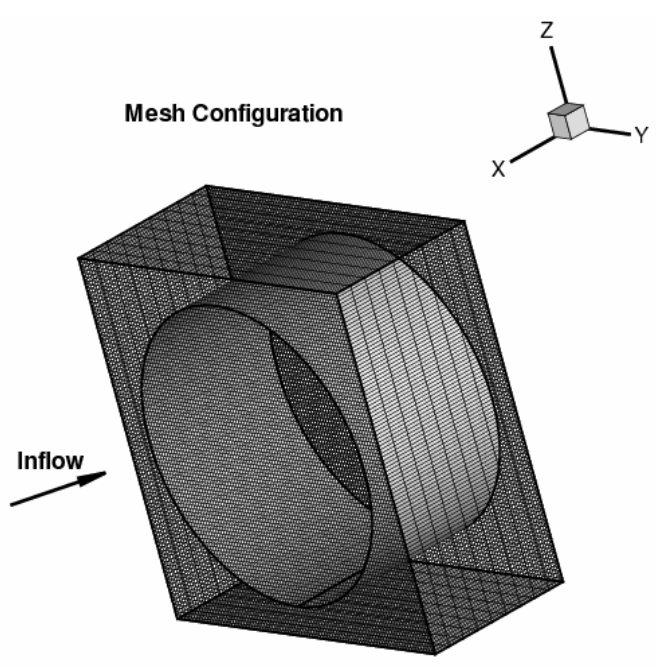

Case 1 and 2: Pipe flow simulation with Cartesian grid using immersed boundary method (pipe outside is immersed region).

Fig. 3-40. Grid layout for pipe flow validation case using immersed boundary method.

A view of the grid in one cross section is shown in Figure 3-41. A comparison of the computed velocity profile and the analytical solution for fully-developed laminar flow is shown in Figure 3-42. The streamwise mean 
velocity distribution for fully-developed turbulent pipe flow using the immersed boundary method is compared with the results obtained by Xu et al. [2005] using a cylindrical-coordinate, body-fitted method in Figure 3-43. Both cases demonstrate good agreement with the reference data.

The two-rod complex flow configuration (Task 5) is shown in Figure 3-44. The LES simulations assumed fully-developed flow and applied step-periodic conditions in the streamwise direction. Figures 3-45 to 3-47 show measured velocity contours [Smith, 2005] and the results of simulations. The simulations to date have omitted the rod spacers. Figure 3-47 compares measured and computed mean velocity profiles. The agreement is not good, possibly due in part to the neglect of the spacers. We are currently preparing to include spacers in our simulations.

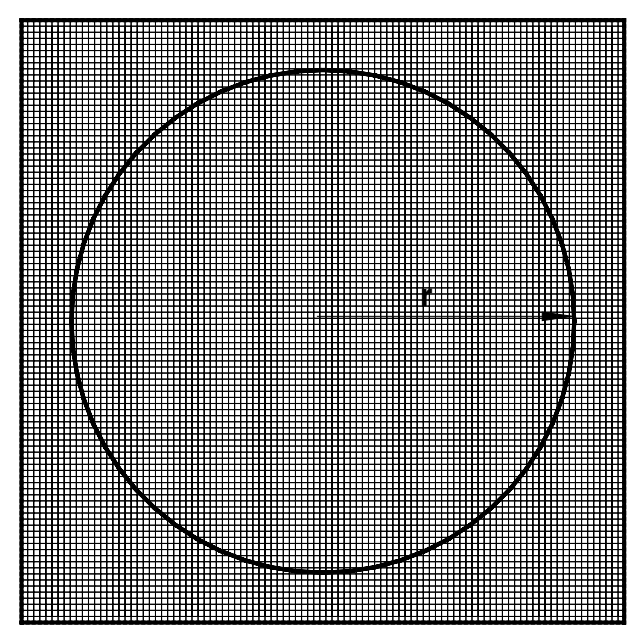

Case 1and 2: Pipe flow simulation with Cartesian grid using immersed boundary method. (pipe outside is immersed region, $2 r / D=0.826$ ).

Fig. 3-41. Grid layout in cross section for pipe flow validation case using immersed boundary method.

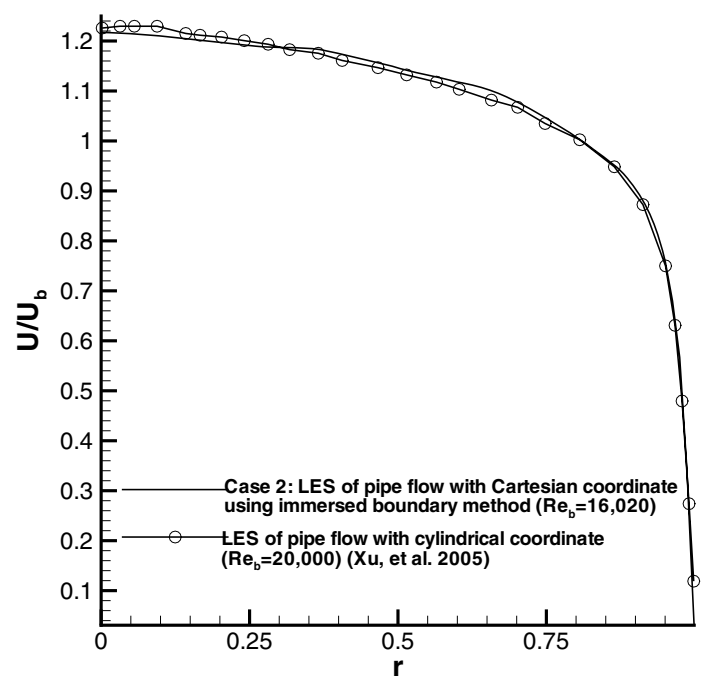

Fig. 3-43. Comparison of streamwise velocity profile from LES with immersed boundary with results of $\mathrm{Xu}$ et al. [2005].

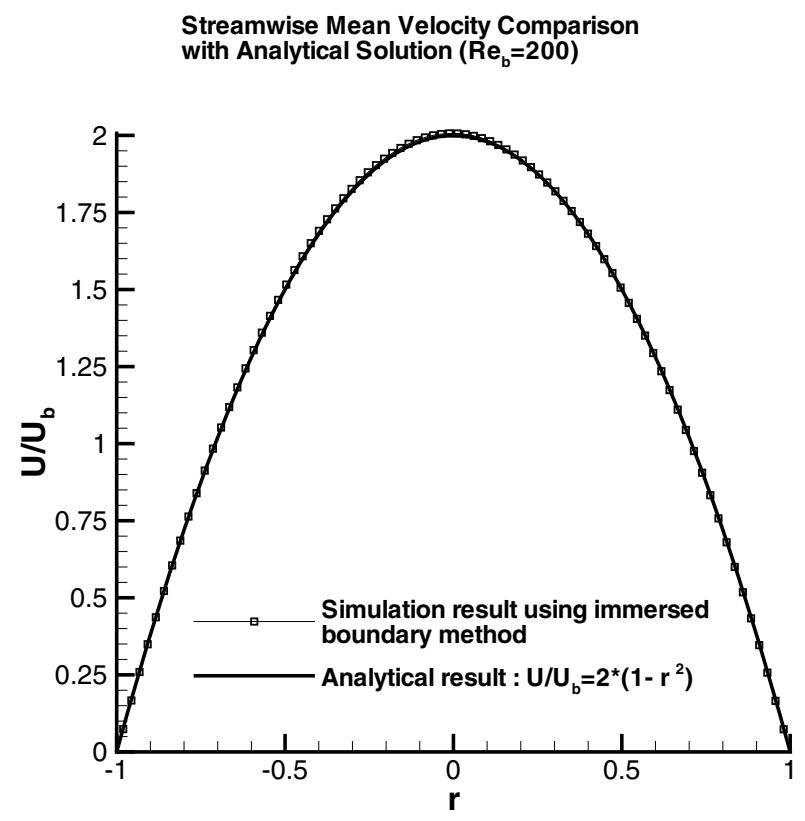

Fig. 3-42. Streamwse velocity profile comparison, laminar validation case using immersed boundary method.

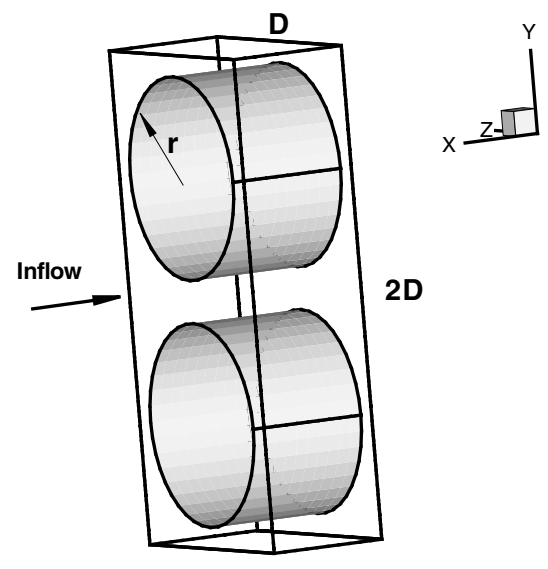

Case 3: Complex geometry : duct flow containing two rods using immersed boundary method. (Interior of rod is immersed region)

Fig. 3-44. Two-rod configuration. 


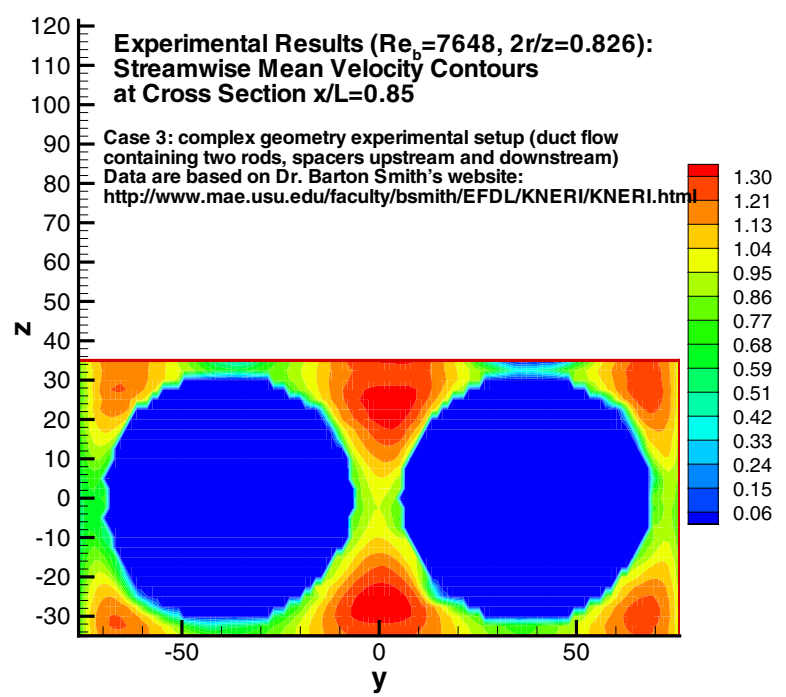

Fig. 3-45. Measured velocity contours, two-rod configuration [Smith, 2005].

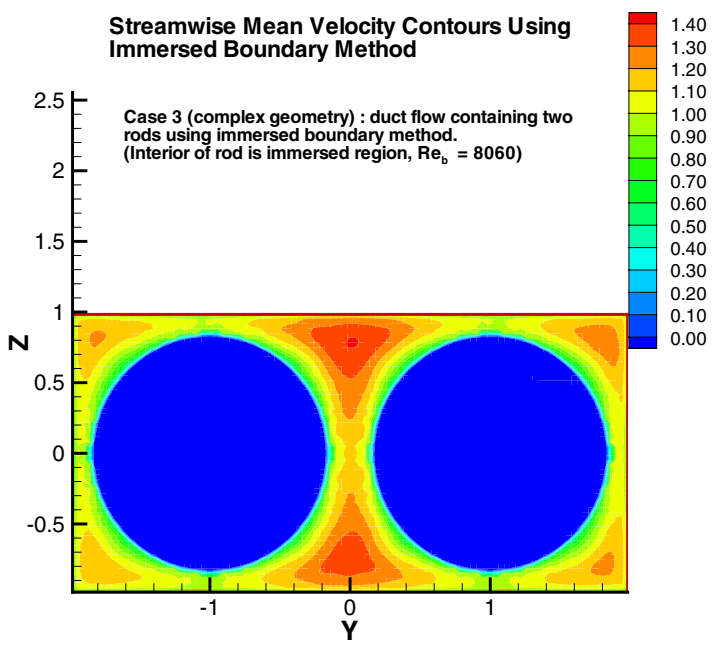

Fig. 3-46. Computed velocity contours, two-rod configuration.

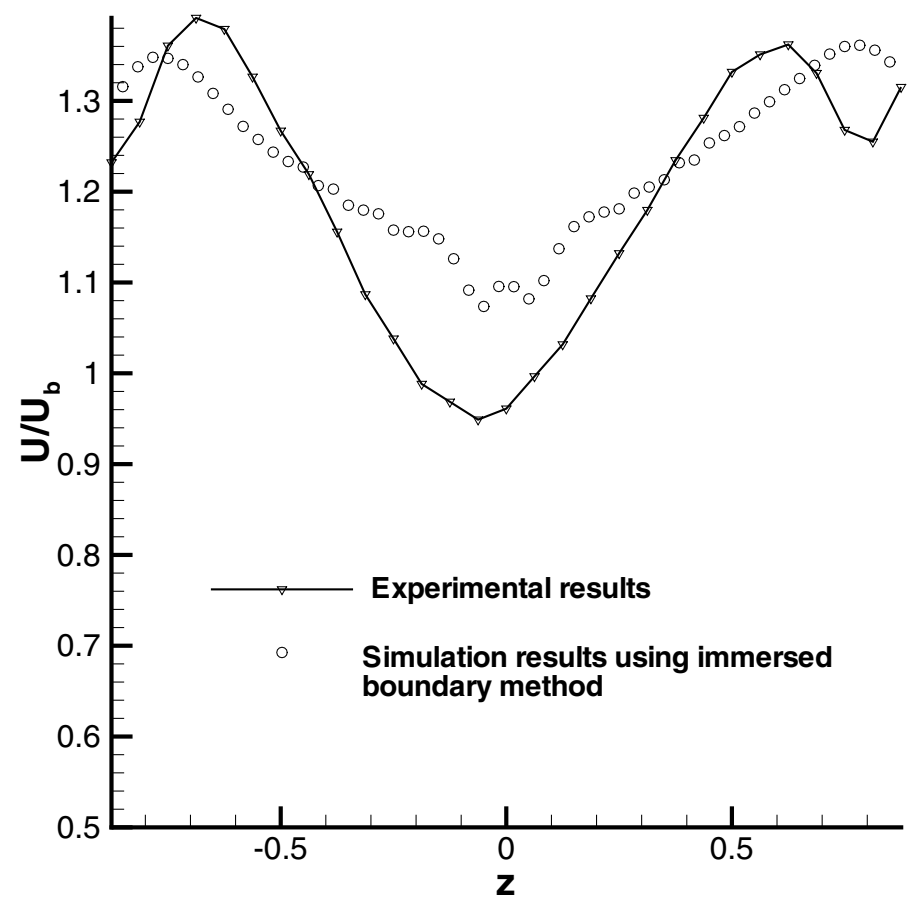

Fig. 3-47. Streamwise mean velocity comparison at $y=0$ plane (Case 3: rod bundle simulation). 
Task 4. Different second-closure (DSM) and Reynolds-averaged Navier-Stokes(RANS) development, Prof. S. O. Park, Korea Advanced Institute of Science and Technology (KAIST)

The objective of this task is assessment of turbulence models in superheated gas flow and supercritical flows with significant property variation in a vertical pipe flow. Various combinations of EASM (Explicit Algebraic Stress model) and EAHFM (Explicit Algebraic heat Flux model) models are adopted for simulation of these flows with property variation. Also a model accounting for the variation of specific heat is developed and the effects of wall distance definition are examined.

4.1. Introduction For heat transfer related to advanced light water and supercritical reactors at normal operating condition, forced convection is dominant along the fuel rod in the fuel assembly. In a Loss of Flow (LOFA) scenario when typically the pump is disabled, the lower cooling mass flux induces strong buoyancy force, which impairs the heat transfer for upward flow in a vertical pipe. The wall temperature abruptly increases at a certain location so that the wall might fail because of high temperature. This local deterioration of heat transfer is reported to be due to the evolutional change of mean velocity profile as flow goes downstream. Kurganov and Kaptilnyi [1992] showed the change of mean velocity profiles during the local heat transfer deterioration experimentally. Detailed information about the general deterioration of heat transfer in a vertical pipe is found in the paper of Jackson, Cotton and Axcell [1989], but the effects of property variation have not reported.

The computations of supercritical flows have been carried out mainly with mixing length models. Hsu and Smith [1961], Hess and Kunz [1965] and Shiralkar and Griffith [1969] employed their own definitions of wall distance coordinates in their mixing length models. Since the properties of working fluids vary near the critical point even within small differences of mean temperature, the wall coordinates have different values at the same physical location according to their definitions. Hall [1971] and Petukhov [1970] compared two different length scales for supercritical flows and recently, Baek and Park [2004] conducted this comparison by using two-equation turbulence models for property-varying flows. Popov and Belyaev [1975] proposed a mixing length scale for low Reynolds number effect in a pipe flow. Later, Popov [1983] developed a new mixing length model that employed a Reynolds shear stress correction for property-variation effects. Valueva and Popov [1985] showed that this model resulted in good agreement to subcritical and supercritical flows. On the other hand, Bellmore and Reid [1983] suggested a mixing length model with density fluctuation term, which was derived from the Reynolds-average technique rather than from the Favre-average technique. Lee and Howell [1998] modified this model and showed that their results were in a good agreement with some data.

With Jones amd Launder's [1973] two-equation turbulence model, Koshizuka, Takano and Oka [1995] conducted a numerical analysis of deterioration phenomena in heat transfer for supercritical water. Li, lin and Ebadian[1999] investigated developing turbulent flow and heat transfer characteristics of water near the critical point in a curved pipe by using the RNG $k-\varepsilon$ model with a commercial code. He et al. [2003] assessed the prediction performance of a number of two-equation, low-Reynolds-number turbulence models and showed that the performance of the models varied significantly from one to another.

For two-equation turbulence models, no model reflecting property-variation effects for supercritical flows has yet been reported. In this paper we look into some related issues. First, we examine how well the turbulence models perform for property-varying flows. Second, we consider the definition of specific heat capacity and derive a new term including the effect of property variation. The underlying assumption is that the correlation with the fluctuation of specific heat capacity is not negligible. From this idea, we develop a new turbulent heat-flux model to be used in conjunction with a conventional two-equation turbulence model. Third, we mention other issues for further study.

4-2. DSM and RANS development For the prediction of turbulent convective flow with significant buoyancy effects, the combination of algebraic stress and flux models seems to be the minimum requirement to represent the anisotropy of the flow and the interaction between the momentum and the buoyancy forces adequately. The EASM (Explicit Algebraic Stress model) can represent the anisotropy and the AHFM (Algebraic Heat Flux Model) is not affected by the temperature gradient directly. Combination of algebraic models requires only three or four differential equations for turbulence quantities while the DSM combination has eleven differential equations for three-dimensional flow. The use of an algebraic stress model reduces computational efforts dramatically, keeping 
the characteristics of DSM closures. Due to their simplicity and less computational time, many industrial engineers prefer the algebraic model combination for practical applications. Hence, the present work is concentrated on the numerical simulation with the combination of algebraic models.

4-2-1. Governing equations and numerical scheme We deal with steady axisymmetric flow in a vertical tube as sketched in Figure 4-1. Since the Mach number is very small, we neglect the viscous dissipation term and the pressure effect in the energy equation. We consider the buoyancy effects in the momentum equation and in the turbulence model equations. We assume that the fluctuations of the thermal properties are sufficiently small compared to their mean values so that we may neglect the terms including the fluctuations of thermal properties. With these assumptions, the governing equations are given as follows.

Continuity:

$$
\partial_{j}\left(\bar{\rho} \bar{u}_{j}\right)=0
$$

Momentum:

$$
\partial_{j}\left(\bar{\rho} \bar{u}_{j} \bar{u}_{i}\right)=\partial_{j}\left[\bar{\tau}_{i j}-\overline{\rho u_{i}^{\prime} u_{j}^{\prime}}\right]-\partial_{i} P+\bar{\rho} g_{i}
$$

Energy:

$$
\partial_{j}\left(\bar{\rho} \bar{u}_{j} \bar{h}\right)=\partial_{j}\left[(\mu / P r) \partial_{j} \bar{h}-\overline{\rho u_{j}^{\prime} h^{\prime}}\right]
$$

Turbulence kinetic energy:

$$
\partial_{j}\left(\bar{\rho} \bar{u}_{j} k\right)=\partial_{j}\left[\left(\mu+\mu_{t} / \sigma_{k}\right) \partial_{j} k\right]-\overline{\rho u_{i}^{\prime} u_{j}^{\prime}} \tilde{u}_{i, j}-\bar{\rho} \beta g_{j} \overline{u_{j}^{\prime} T^{\prime}}-\bar{\rho} \varepsilon
$$

Dissipation rate of turbulence kinetic energy:

$$
\partial_{j}\left(\bar{\rho} \bar{u}_{j} \varepsilon\right)=\partial_{j}\left[\left(\mu+\mu_{t} / \sigma_{\varepsilon}\right) \partial_{j} \varepsilon\right]-C_{\varepsilon 1} f_{1} \frac{\varepsilon}{k}\left(\overline{\rho u_{i}^{\prime} u_{j}^{\prime}} \bar{u}_{i, j}+\bar{\rho} \beta g_{j} \overline{u_{j}^{\prime} T^{\prime}}\right)-\bar{\rho} C_{\varepsilon 2} f_{2} \frac{\varepsilon^{2}}{k}+\bar{\rho} E
$$

where, the molecular stress tensor, $\bar{\tau}_{i j}$, is given by

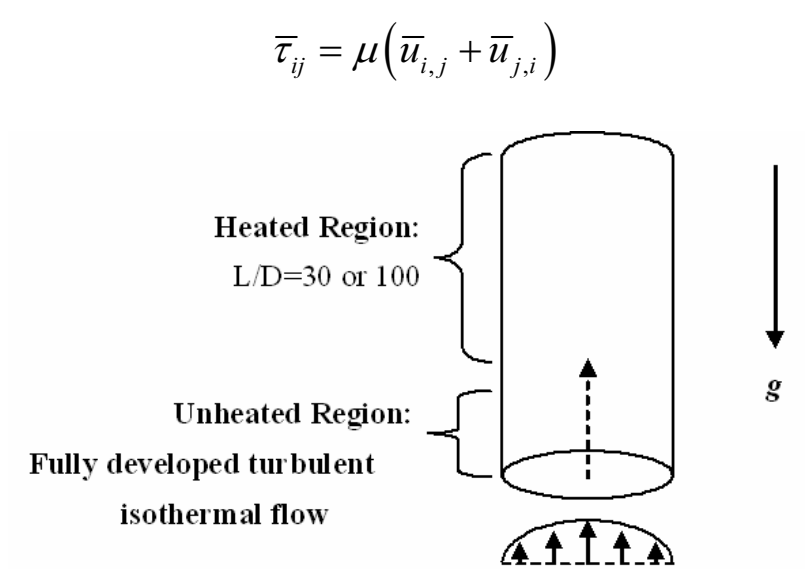

Fig. 4-1. Schematic diagram of computational configuration 
The governing equations are discretized using a second-order finite volume method in the present study, employing the QUICK scheme for the convective term in Equations. (4-2) to (4-5). Other terms were discretized by second-order central differences, keeping the conservative form. In conjunction with the momentum equations, the continuity equation was converted to a pressure-correction equation, which was solved using the SIMPLE algorithm. The set of algebraic equations is solved with Stone's strongly implicit scheme. The convergence criteria for the residuals of all equations were assumed to be less than $10^{-6}$ of their total inflow rates.

For the physical properties of the working fluid varying with temperature, we use the subroutine PROPATH [2001] which was distributed by Dr. Takata of Kyushu University. The transport property relations are in the following form.

$$
\rho=\rho(P, T), \mu=\mu(P, T), \operatorname{Pr}=\operatorname{Pr}(P, T), T=T(P, h)
$$

The thermal properties were estimated at every iteration by using PROPATH. Figure 4-1 shows a schematic diagram of the flow region and the boundary conditions in the present calculation. The inflow condition is the fully developed turbulent isothermal flow. For velocity at the wall, the non-slip condition is employed. The thermal condition at the wall is taken to be adiabatic for the first five diameters, followed by a specified uniform wall heat flux. At the outlet, we set the second order derivatives of variables to be zero.

\section{4-2-2. Turbulence models}

\section{4-2-2-1. Modeling of Reynolds stress.}

\section{(1) Linear eddy viscosity model}

Chien (CH) \& Launder-Sharma (LS) The Reynolds stress models of Chien [1982] and Launder and Sharma [1974] are based on the linear eddy-viscosity model.

$$
\overline{\rho u_{i}^{\prime} u_{j}^{\prime}}=\frac{2}{3} \bar{\rho} k \delta_{i j}-2 \mu_{t} S_{i j}
$$

with

$$
\mu_{t}=\bar{\rho} C_{\mu} f_{\mu} k^{2} / \varepsilon
$$

where, the model constant and the damping function are given in Table 4-1 to Table 4-3.

Table 4-1. Model constants for $k-\varepsilon$ models.

\begin{tabular}{|c|c|c|c|c|c|}
\hline Model & $C_{\mu}$ & $C_{\varepsilon 1}$ & $C_{\varepsilon 2}$ & $\sigma_{k}$ & $\sigma_{\varepsilon}$ \\
\hline $\mathrm{LS}$ & 0.09 & 1.44 & 1.92 & 1.0 & 1.3 \\
\hline $\mathrm{CH}$ & 0.09 & 1.35 & 1.80 & 1.0 & 1.3 \\
\hline $\mathrm{WJ}$ & 0.09 & 1.35 & 1.80 & 1.0 & 1.3 \\
\hline $\mathrm{GS}$ & 0.088 & 1.39 & 1.83 & 1.0 & 1.3 \\
\hline
\end{tabular}


Table 4-2. Damping functions for $k-\varepsilon$ models.

\begin{tabular}{|c|c|c|c|}
\hline Model & $f_{\mu}$ & $f_{1}$ & $f_{2}$ \\
\hline LS & $\exp \left[-3.4 /\left(1+\mathrm{Re}_{t} / 50\right)^{2}\right]$ & 1 & $1-0.3 \exp \left(-\mathrm{Re}_{t}^{2}\right)$ \\
\hline CH & $1-\exp \left(-0.0115 y^{+}\right)$ & 1 & $1-0.22 \exp \left(-\mathrm{Re}_{t}^{2} / 36\right)$ \\
\hline WJ & $1-\exp \left(-0.0115 y^{+}\right)$ & 1 & $1-0.22 \exp \left(-\mathrm{Re}_{t}^{2} / 36\right)$ \\
\hline GS & 1 & 1 & {$\left[1-\exp \left(-y^{+} / 6.4\right)\right]^{2}$} \\
\hline
\end{tabular}

Table 4-3: Extra terms and other features for $k-\varepsilon$ models.

\begin{tabular}{|c|c|c|c|}
\hline Model & $\mathrm{D}$ & $\mathrm{E}$ & BC for $\varepsilon$ at walls \\
\hline LS & $2 v(\partial \sqrt{k} / \partial y)^{2}$ & $2 v v_{t}\left(\partial^{2} u / \partial y^{2}\right)^{2}$ & 0 \\
\hline CH & $2 v k / y^{2}$ & $-\left(2 v \varepsilon / y^{2}\right) \exp \left(-0.5 y^{+}\right)$ & 0 \\
\hline WJ & $2 v k / y^{2}\left(-0.04 y^{+}\right)$ & $-\left(2 v \varepsilon / y^{2}\right) \exp \left(-0.5 y^{+}\right)$ & $2 v\left(\partial \sqrt{k} / \partial x_{k}\right)$ \\
\hline GS & 0 & 0 & 0 \\
\hline
\end{tabular}

\section{(2) Explicit algebraic stress model}

Gatski-Speziale (GS)

Gatski and Speziale [1993] proposed an explicit algebraic stress model for the first time. Abid, Rumsey and Eatski [1995] modified the model to overcome the numerical instability of the original model. The explicit nonlinear constitutive equation is given by

$$
\begin{aligned}
\overline{\rho u_{i}^{\prime} u_{j}^{\prime}} & =\frac{2}{3} \bar{\rho} k \delta_{i j}-2 \mu_{t}\left[\left(S_{i j}-\frac{1}{3} S_{k k} \delta_{i j}\right)\right. \\
& \left.+\left(\alpha_{4} / \omega\right)\left(S_{i k} W_{k j}+S_{j k} W_{k i}\right)-\left(\alpha_{5} / \omega\right)\left(S_{i k} S_{k j}-\frac{1}{3} S_{k l} S_{k l} \delta_{i j}\right)\right]
\end{aligned}
$$

with

$$
\mu_{t}=\bar{\rho} C_{\mu}^{*}(k / \omega) \text { and } C_{\mu}^{*}=\frac{3\left(1+\eta^{2}\right) \alpha_{1}}{3+\eta^{2}+6 \eta^{2} \xi^{2}+6 \xi^{2}}
$$

where,

$$
\eta^{2}=\left(\alpha_{2} / \omega^{2}\right)\left(S_{i j} S_{i j}\right) \text { and } \xi^{2}=\left(\alpha_{3} / \omega^{2}\right)\left(W_{i j} W_{i j}\right)
$$

Here, $\omega=\varepsilon / k$ is the specific dissipation rate, $S_{i j}=0.5\left(\bar{u}_{i, j}+\bar{u}_{j, i}\right)$, and $W_{i j}=0.5\left(\bar{u}_{i, j}-\bar{u}_{j, i}\right)$. The constants in Eqs. (4-10)-(4-12) are given by

$$
\begin{gathered}
\alpha_{1}=\left(\frac{4}{3}-C_{2}\right)(g / 2), \alpha_{2}=\left(2-C_{3}\right)^{2}\left(g^{2} / 4\right), \alpha_{3}=\left(2-C_{4}\right)^{2}\left(g^{2} / 4\right) \\
\alpha_{4}=\left(\frac{2-C_{4}}{2}\right) g, \alpha_{5}=\left(2-C_{3}\right) g, g=\frac{1}{\left(C_{1} / 2\right)+C_{5}-1}
\end{gathered}
$$




$$
C_{1}=3, C_{2}=0.8, C_{3}=1.75, C_{4}=1.31, C_{5}=2
$$

Equation (4-10) is solved together with Equations (4-4) and (4-5), as suggested by Abid, Rumsey and Gatski. [1995]. The model constants and damping functions for Equations (4-4) and (4-5) are also given in Table 4-1.

Wallin-Johansson (WJ) Wallin and Johansson [2000] proposed an explicit algebraic stress model with the nearwall correction that represented an exact solution of the implicit algebraic Reynolds stress model. The models are as follows:

$$
\overline{\rho u_{i}^{\prime} u_{j}^{\prime}}=\frac{2}{3} \bar{\rho} k \delta_{i j}-2 \mu_{t}\left(S_{i j}-\frac{1}{3} S_{k k} \delta_{i j}\right)+\bar{\rho} \tau^{2} k a_{i j}
$$

with

$$
\mu_{t}=-\frac{1}{2} \bar{\rho} \tau k f_{1} \beta_{1}
$$

and

$$
a_{i j}=\frac{\left(3 B_{2}-4\right)\left(1-f_{1}^{2}\right)}{\max \left(I I_{S}, I I_{S}^{e q}\right)}\left(S_{i k} S_{k j}-\frac{1}{3} I I_{S} \delta_{i j}\right)+\left[f_{1}^{2} \beta_{4}-\frac{B_{2}\left(1-f_{1}^{2}\right)}{2 \max \left(I I_{S}, I I_{S}^{e q}\right)}\right]\left(S_{i k} W_{k j}+S_{j k} W_{k i}\right)
$$

where the turbulent time scale is defined by

$$
\tau=\max \left(k / \varepsilon, C_{\tau} \sqrt{v / \varepsilon}\right)
$$

The $\beta$-coefficients are given by

$$
\beta_{1}=-\frac{6}{5} N /\left(N^{2}-2 I I_{\Omega}\right) \text { and } \beta_{4}=-\frac{6}{5} N /\left(N^{2}-2 I I_{\Omega}\right) .
$$

and the invariants are defined by

$$
I I_{S}=\tau^{2} S_{i j} S_{j i}, I I_{\Omega}=\tau^{2} W_{i j} W_{j i}, \text { and } I I_{S}^{e q}=405 c_{1}^{2} /\left(216 c_{1}-160\right)
$$

The quantity $N$ is closely related to the production to dissipation rate and is given by

$$
N= \begin{cases}c_{1}^{\prime} / 3+\left(P_{1}+\sqrt{P_{2}}\right)^{1 / 3}+\operatorname{sign}\left(P_{1}-\sqrt{P_{2}}\right)\left|P_{1}-\sqrt{P_{2}}\right|^{1 / 3}, & P_{2} \geq 0 \\ c_{1}^{\prime} / 3+2\left(P_{1}^{2}-P_{2}\right)^{1 / 6} \cos \left(\frac{1}{3} \operatorname{arcos}\left(P_{1} / \sqrt{P_{1}^{2}-P_{2}}\right)\right), & P_{2}<0\end{cases}
$$

with

$$
P_{1}=\left(c_{1}^{\prime 2} / 27+\frac{9}{20} I I_{S}-\frac{2}{3} I I_{\Omega}\right) c_{1}^{\prime} \text { and } P_{2}=P_{1}^{2}-\left(\frac{1}{9} c_{1}^{\prime 2}+\frac{9}{19} I I_{S}+\frac{2}{3} I I_{\Omega}\right)^{3}
$$

and 


$$
c_{1}^{\prime}=\frac{9}{4}\left[c_{1}-1+C_{D} \max \left(1+\beta_{1}^{e q} I I_{S}, 0\right)\right]
$$

where

$$
\beta_{1}^{e q}=-\frac{6}{5} N^{e q} /\left(\left(N^{e q}\right)^{2}-2 I I_{\Omega}\right) \text { and } N^{e q}=\frac{9}{4} c_{1}
$$

The damping function reads

$$
f_{1}=1-\exp \left(-C_{y 1}^{\prime} \sqrt{R e_{y}}-C_{y 2}^{\prime} R e_{y}^{2}\right)
$$

where $R e_{y}=\sqrt{k} y / v$ and the coefficients are given by

$$
C_{\tau}=6.0, c_{1}=1.8, B_{2}=1.8, C_{y 1}^{\prime}=2.4 / 26.0, \text { and } C_{y 2}^{\prime}=0.003 / 26.0
$$

The base-line $k-\varepsilon$ model is the low-Reynolds number model of Chien [1982]. Wallin and Johansson modified the dissipation length scale near the wall to match the logarithmic "law" for a channel flow. The functions and model constants are given Table 4-1.

\section{4-2-2-2. Explicit(implicit) algebraic heat-flux model}

Abe-Kondoh-Nagano (AKN) Abe, Kondoh and Nagano [1996] proposed an explicit algebraic heat-flux model with low-Reynolds number effects in the near-wall region. The constitutive relations are as follows:

$$
\overline{u_{j}^{\prime} \theta}=\frac{C_{T 1} \tau_{m}}{1+\frac{1}{2} \tau_{m}^{2}\left(c_{S}^{2} S_{k l} S_{k l}+c_{\Omega}^{2} W_{k l} W_{k l}\right)}\left\{-\delta_{j l}+\tau_{m}\left(c_{S} S_{j l}+c_{\Omega} W_{j l}\right)\right\} \overline{u_{l}^{\prime} u_{k}^{\prime}} \partial_{k} \Theta
$$

The characteristic time scale for heat transfer is given by

$$
\tau_{m}=(k / \varepsilon) f_{R} f_{A} f_{\lambda} f_{w}
$$

where the damping functions are:

$$
\begin{gathered}
f_{R}=2 R /(0.5+R), f_{A}=2 /\left(1+3.5 \sqrt{b_{i j} b_{i j}}\right) \\
f_{\lambda}=1+24\left(\operatorname{Re}_{t}^{3 / 4} f_{R} f_{A}\right)^{-1}(2 R / \operatorname{Pr})^{1 / 2} \exp \left[-\left(\operatorname{Re} e_{t} / 30\right)^{3 / 4}\right] \\
f_{w}=1-f_{w}(26)
\end{gathered}
$$

with

$$
b_{i j}=\overline{u_{i}^{\prime} u_{j}^{\prime}} / 2 k-\delta_{i j} / 3, R=\left(k_{\theta} / \varepsilon_{\theta}\right) /(k / \varepsilon)
$$


Modified AKN (mAKN) To check the near-wall behavior of $\overline{v^{\prime} \theta}$ of the AKN explicit heat-flux model, we apply the AKN model to two-dimensional fully-developed flow. Then, the simplified heat-flux in the normal direction becomes

$$
\overline{v^{\prime} \theta}=\frac{C_{T 1} \tau_{m}}{1+C_{T 2} C_{T 3}\left(\tau_{m} \bar{u}_{1,2}\right)^{2}}\left\{-\overline{v^{\prime 2}}+C_{T 3} \tau_{m} P_{k}\right\} \Theta_{2}
$$

Assuming that the near-wall behaviors of scalar variables (such as $k, \varepsilon$ and $P_{k}$ ) are correct, we find that, as $y$ approaches zero, $f_{R}$ and $f_{A}$ become constant, $f_{\lambda}$ is proportional to $y^{-3}$ and $f_{w}$ is asymptotic to $y^{2}$. Thus, the time scale, $\tau_{m}$, is proportional to $y$. From these near-wall behaviors, the asymptotic behavior of $\overline{v^{\prime} \theta}$ at the wall is

$$
\overline{v^{\prime} \theta} \propto \tau_{m} /\left(1+y^{2}\right) \cdot y^{4} \propto y^{5}
$$

which deviates from the theoretical near-wall behavior of $O\left(y^{3}\right)$. To correct the near-wall behavior of turbulent heat flux in the normal direction we redefine the time scale, $\tau_{m}^{\prime}$, only in the numerator except for the time scale with mean strain rate tensor.

$$
\tau_{m}^{\prime}=(k / \varepsilon) f_{R} f_{A} f_{\lambda}
$$

Then the near-wall behavior of $\tau_{m}^{\prime}$ is $O\left(y^{-1}\right)$ and the asymptotic behavior of $\overline{v^{\prime} \theta}$ of Equation (4-32) at the wall becomes $\overline{v^{\prime} \theta} \propto \tau_{m}^{\prime} /\left(1+y^{2}\right) \cdot y^{4} \propto y^{3}$.

The final expression leads to

$$
\overline{u_{j}^{\prime} \theta}=\frac{C_{T 1} \tau_{m}^{\prime}}{1+\frac{1}{2} \tau_{m}^{2}\left(c_{S}^{2} S_{k l} S_{k l}+c_{\Omega}^{2} W_{k l} W_{k l}\right)}\left\{-\delta_{j l}+\tau_{m}\left(c_{S} S_{j l}+c_{\Omega} W_{j l}\right)\right\} \overline{u_{l}^{\prime} u_{k}^{\prime}} \partial_{k} \Theta
$$

Suga-Abe (SA) Suga and Abe [2000] proposed a heat-flux model that can be combined with nonlinear eddyviscosity models or second-moment closures. Their algebraic turbulent heat-flux model consists of symmetric and asymmetric parts as follows:

$$
\overline{u_{j}^{\prime} \theta}=-c_{\theta} k \tau\left(\sigma_{j k}+\alpha_{j k}\right) \Theta_{k}
$$

where the symmetric and the asymmetric parts are, respectively, given by

$$
\begin{gathered}
\sigma_{i j}=c_{\sigma 0} \delta_{i j}+c_{\sigma 1} \overline{u_{i}^{\prime} u_{j}^{\prime}} / k+c_{\sigma 2} \overline{u_{i}^{\prime} u_{k}^{\prime}} \cdot \overline{u_{k}^{\prime} u_{j}^{\prime}} / k^{2} \\
\alpha_{i j}=c_{\alpha 0} \tau W_{i j}+c_{\alpha 1} \tau\left(W_{i k} \overline{u_{k}^{\prime} u_{j}^{\prime}} / k+W_{k j} \overline{u_{i}^{\prime} u_{k}^{\prime}} / k\right)
\end{gathered}
$$

with

$$
\tau=f_{t} k / \varepsilon
$$


Kenjeres-Hanjalic (KH) Kenjeres and Hanjalic [2002] suggested an implicit algebraic expression for turbulent heat-flux as follows:

$$
\overline{u_{j}^{\prime} \theta}=-c^{\theta} \tau\left(\overline{u_{j}^{\prime} u_{k}^{\prime}} \partial_{k} \Theta+\xi \overline{u_{k}^{\prime} \theta} \cdot \partial_{k} \tilde{u}_{j}+2 \eta g_{j} \beta k_{\theta}\right)
$$

where

$$
\tau=k / \varepsilon, c^{\theta}=0.2, \xi=0.6, \eta=0.6
$$

For the Reynolds analogy, the turbulent heat fluxes, $\overline{\rho u_{j}^{\prime} h^{\prime}}$ in Equations (4-3) and $\overline{\rho u_{j}^{\prime} T^{\prime}}$ in Equations (4-4) and (4-5), are given by

$$
-\overline{\rho u_{j}^{\prime} h^{\prime}}=\left(\mu_{t} / \operatorname{Pr}_{t}\right) \partial_{j} \tilde{h} \text { and }-\overline{\rho u_{j}^{\prime} T^{\prime}}=\left(\mu_{t} / \operatorname{Pr}_{t}\right) \partial_{j} \tilde{T}
$$

For turbulent Prandtl number, $\operatorname{Pr}_{t}$, we adopt the relation suggested by Kays and Crawford [1993).

$$
\operatorname{Pr}_{t}=\left[\frac{1}{2 \operatorname{Pr}_{t \infty}}+\frac{c \mathrm{Pe}_{t}}{\sqrt{\operatorname{Pr}_{t \infty}}}-\left(c \operatorname{Pe}_{t}\right)^{2}\left\{1-\exp \left(\frac{-1}{c \operatorname{Pe}_{t} \sqrt{\operatorname{Pr}_{t \infty}}}\right)\right\}\right]^{-1}
$$

where $\operatorname{Pe}_{t}=\left(\mu_{t} / \mu\right) \operatorname{Pr}, \operatorname{Pr}_{t \infty}$ is the value of $\operatorname{Pr}_{t}$ far from the wall $(=0.85)$ and $c$ is an empirical constant $(=0.3)$. The nondimensional variables used in damping functions of turbulence models are set to employ the "local" physical properties rather than the wall properties.

$$
y^{+}=\sqrt{\rho \tau_{w}} y / \mu, R e_{t}=\rho k^{2} / \mu \varepsilon \text { and } R e_{y}=\rho \sqrt{k} y / \mu
$$

When a combination model of EASM and EAHFM is applied, some EAHFM require the thermal turbulence length and time scales and others do not. The equations used for thermal turbulence scales are:

Temperature variance:

$$
\partial_{j}\left(\bar{\rho} \bar{u}_{j} k_{\theta}\right)=\partial_{j}\left[\left(\lambda+\lambda_{t} / \sigma_{h}\right) \partial_{j} k_{\theta}\right]-\overline{\rho u_{j}^{\prime} h^{\prime}} \partial_{j} \bar{h}-\bar{\rho} \varepsilon_{\theta}
$$

Dissipation rate of temperature variance:

$$
\begin{aligned}
\partial_{j}\left(\bar{\rho} \bar{u}_{j} \bar{\varepsilon}_{\theta}\right)= & \partial_{j}\left[\left(\lambda+\lambda_{t} / \sigma_{\phi}\right) \partial_{j} \bar{\varepsilon}_{\theta}\right]-\frac{1}{2} C_{P 1} f_{P 1}\left(\bar{\varepsilon}_{\theta} / k_{\theta}\right) \overline{\rho u_{j}^{\prime} h^{\prime}} \partial_{j} \bar{h} \\
& -C_{P 2} f_{P 2}\left(\bar{\varepsilon}_{\theta} / k\right) \overline{\rho u_{i}^{\prime} u_{j}^{\prime}} \partial_{j} \tilde{u}_{i}-\frac{1}{2} \bar{\rho} C_{D 1} f_{D 1}\left(\bar{\varepsilon}_{\theta}^{2} / k_{\theta}\right)-\bar{\rho} C_{D 2} f_{D 2} \bar{\varepsilon}_{\theta}(\varepsilon / k)+\bar{\rho} E_{\theta}
\end{aligned}
$$

where

$$
\lambda_{t}=c_{p} \alpha_{t}, \alpha_{t}=C_{\lambda} f_{t} \tau k, \text { and } \tilde{\varepsilon}_{\theta}=\varepsilon_{\theta}-D_{\theta}
$$

4-2-3. Comparisons of DSM and RANS models Twelve individual sets of experimental data for wall temperature or Nusselt number variation are compared here: the data are taken from the papers of Shehata and McEligot [1998], Vilemas, Poskas and Kaupas (VPK) [1992], Kurganov and Kaptilnyi (KK) [1992], and Weinburg 
(W) [1972]. The data for the VPK series and W series are obtained from the papers of Cotton, Ismael and Kirwin [2001] and He et al. [2003], respectively. Table 4-4 details the values of various parameters at the start of heating in these cases. For superheated air flows, six experimental cases were selected to examine the model performance. For supercritical flows, in two test cases (W1 and W2) the pseudo-critical temperature is not realized and, therefore, the heat transfer in this case is similar with that of superheated gas flow. In the other $\mathrm{CO}_{2}$ cases the pseudo-critical temperature occurs near the wall and the wall temperature has a local maximum near the start of heating, a different phenomenon with that in the gas flow and not clearly explained yet.

Eight combinations of explicit algebraic stress (GS and WJ) and heat-flux models (AKN, $m \mathrm{AKN}, \mathrm{KH}$, and $\mathrm{SA}$ ) and two low-Reynolds-number, $k-\varepsilon$ turbulence models (LS and $\mathrm{CH}$ ) are applied to these test cases.

Table 4-4: Conditions of the start of heating in experiments

\begin{tabular}{cccccccc}
\hline Medium & Test Case & $\boldsymbol{P}_{\boldsymbol{i n}}(\boldsymbol{M P a})$ & $\boldsymbol{T}_{\boldsymbol{i n}}(\boldsymbol{K})$ & $\boldsymbol{R e}_{\boldsymbol{d}}$ & $\boldsymbol{q}_{\boldsymbol{i n}}^{+}$ & $\boldsymbol{G r}_{\boldsymbol{q}, \boldsymbol{i n}}$ & $\boldsymbol{P r}_{\boldsymbol{i n}}$ \\
\hline \multirow{6}{*}{ Air } & RUN445 & 0.092 & 297.5 & 4280 & $4.5 \times 10^{-3}$ & $9.39 \times 10^{6}$ & 0.713 \\
& RUN635 & 0.092 & 296.6 & 6050 & $3.5 \times 10^{-3}$ & $1.04 \times 10^{7}$ & 0.713 \\
& RUN618 & 0.093 & 296.0 & 6080 & $1.8 \times 10^{-3}$ & $5.56 \times 10^{6}$ & 0.713 \\
\cline { 2 - 8 } & VPK10 & 0.7 & 294.0 & 7280 & $2.66 \times 10^{-3}$ & $1.44 \times 10^{9}$ & 0.717 \\
& VPK11 & 0.7 & 294.0 & 11400 & $2.18 \times 10^{-3}$ & $1.72 \times 10^{9}$ & 0.717 \\
& VPK12 & 0.7 & 294.0 & 20700 & $1.68 \times 10^{-3}$ & $2.40 \times 10^{9}$ & 0.717 \\
\hline \multirow{6}{*}{$\mathrm{CO}_{2}$} & W1 & 0.76 & 283.2 & 59340 & $4.47 \times 10^{-5}$ & $3.68 \times 10^{10}$ & 2.19 \\
& W2 & 0.76 & 283.0 & 40520 & $4.93 \times 10^{-5}$ & $2.63 \times 10^{10}$ & 2.19 \\
& W5 & 0.76 & 283.2 & 29670 & $1.47 \times 10^{-4}$ & $6.03 \times 10^{10}$ & 2.19 \\
\hline & KK2 & 9.0 & 293.4 & 223300 & $1.36 \times 10^{-4}$ & $8.50 \times 10^{11}$ & 2.25 \\
& KK3 & 9.0 & 293.4 & 224700 & $2.66 \times 10^{-4}$ & $1.68 \times 10^{12}$ & 2.25 \\
& KK4 & 9.0 & 293.4 & 335000 & $2.71 \times 10^{-4}$ & $2.55 \times 10^{12}$ & 2.25 \\
\hline
\end{tabular}

4-2-3-1. Superheated gas flow Figure 4-2 shows the local wall temperature profiles computed by various combinations. In cases of RUN445, RUN635 and RUN618, the predictions of the combinations of the explicit algebraic stress and heat-flux models are not superior to that of the LS model even though they have the ability to describe the anisotropy of Reynolds stresses in nature. All the combinations underpredict the wall temperature development except one case of WJ-AKN in RUN618. In Figure 4-2, WJ-AKN model gives the best agreement with the experimental data. The WJ-combinations (WJ-SA, WJ-KH, WJ-AKN, and WJ-mAKN) that have the corrected near-wall treatments of Reynolds stresses do not yield better predictions than the GS-combinations (GS-KH and GS$\mathrm{AKN}$ ) that do not account for the near-wall behaviors. In addition, compared with the predictions of WJ- $m \mathrm{AKN}$ and WJ-AKN, the modified AKN model (to correct the near-wall behaviors of turbulent heat-flux vector) does not perform better than the original AKN model. These observations suggest that the near-wall behaviors of the Reynolds stresses and the turbulent heat-flux vector may not be crucial.

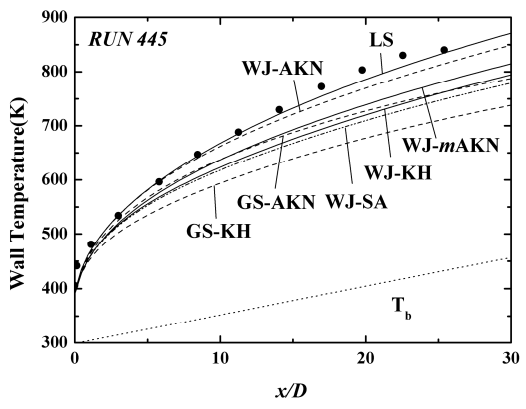

(a)

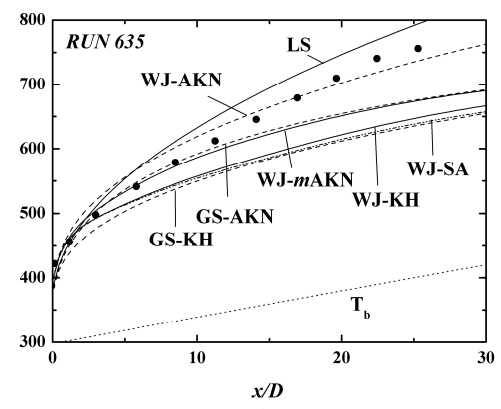

(b)

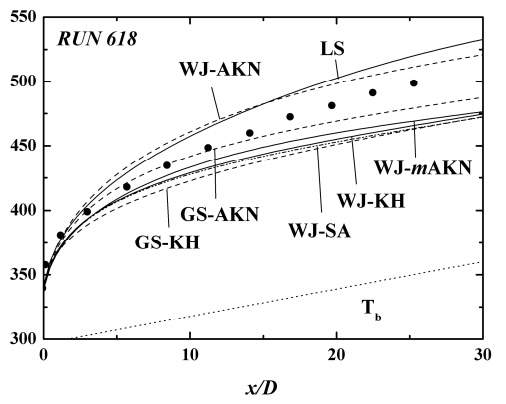

(c)

Fig. 4-2. Wall temperature distributions for superheated gas (air) flows compared with the data of Shehata \& McEligot [1998]. 
Mikielewicz et al. [2002] estimated that for all the test cases of RUN445, RUN635, and RUN618 the acceleration effect was more important than the buoyancy effect. Baek and Park [2003] reconfirmed this assertion for these cases; the turbulence kinetic energy was reduced monotonically along the axis largely due to the thermal acceleration force by the criterion of Vilemas, Roskas and Kaupas [1992]. From these results we conclude that the prediction performance of explicit algebraic models for laminarization depends on the choice of a basic platform, the $k-\varepsilon$ turbulence model.

Figure 4-3 shows the local Nusselt number variations of VPK10, VPK11 and VPK12. These three cases have a local minimum and maximum of Nusselt number, which was due to the change of the mean velocity profile by the buoyancy force. In Figure 4-3(a) and (b), all the models demonstrate a decrease of Nusselt number. In Figure 4-3(c), however, only the LS model shows change of the mean velocity profiles along the streamwise axis (not shown). In Figure 4-3(a) and (b), the explicit algebraic combination model, WJ-mAKN, gives an oscillatory variation of the Nusselt number. Similar oscillatory results appear in Figures 4-4(b)-(c) and Figures 4-5 (b)-(c).

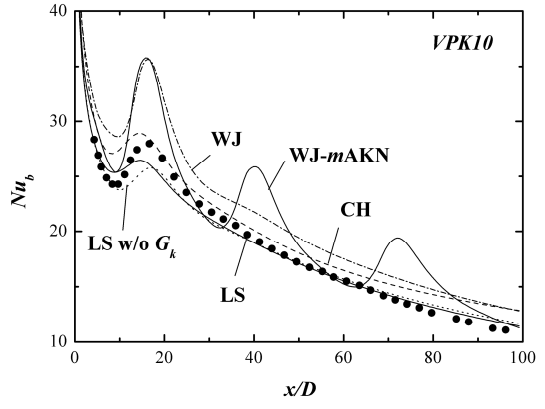

(a)

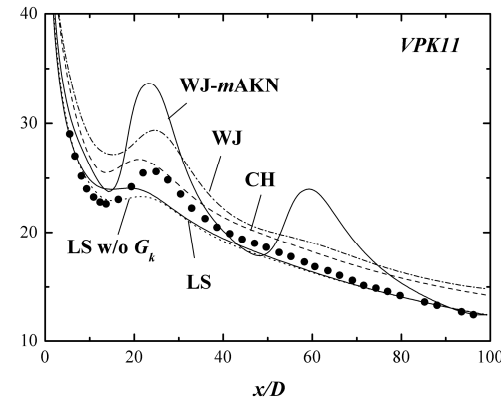

(b)

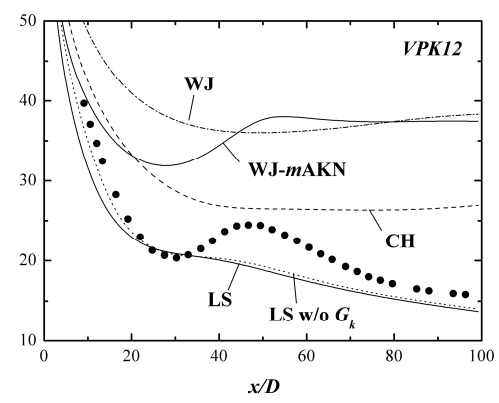

(c)

Fig. 4-3. Nusselt number distributions for superheated gas flows compared with the data of Vilemas et al. [1992]

4-2-3-2. Supercritical flow For the test cases in Figure 4-4, the pseudo-critical temperature is about 32. In Figure 4-4(a) W1 case, the pseudo-temperature does not appear at all and the prediction of LS model is superior to the other models as in superheated gas flows. In Fig. 4-4(b) of W2 case, the wall temperature also does not exceed the pseudo-critical temperature but the buoyancy effect is stronger than in the W1 case. The LS model overpredicts the wall temperature after the sharp peak of the wall temperature, whereas the other models predict the wall temperature well but underpredict the peak temperature near the start of heating. The same results are found in the paper of He et al. [2003]. In Figure 4-4(c) the pseudo-critical temperature is exceeded. The CH, WJ and WJ-mAKN models improve the prediction slightly but the $\mathrm{WJ}-m \mathrm{AKN}$ model gives an oscillatory wall temperature. It is interesting that the $\mathrm{CH}$ model predicts the wall temperature better than the LS model.

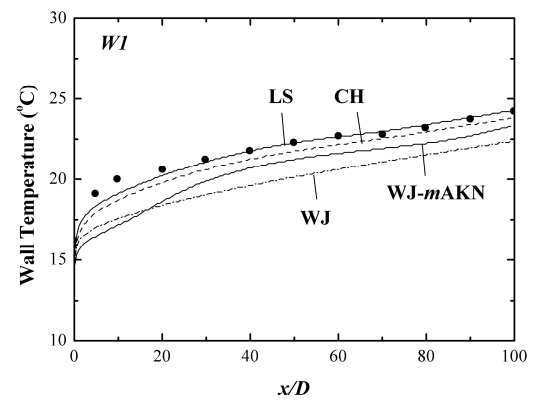

(a)

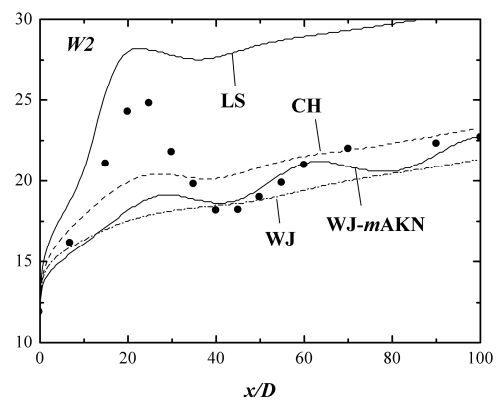

(b)

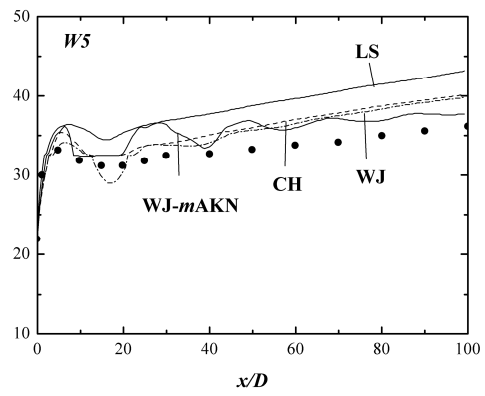

(c)

Fig. 4-4. Wall temperature distributions for supercritical $\mathrm{CO}_{2}$ flows compared with the data of Weinburg [1972] at $\mathrm{p}$ $\approx 0.76 \mathrm{MPa}$. 
While the $\mathrm{W}$-series exhibit small differences between the wall temperature and the bulk temperature, the KKseries show larger differences between the two and experience the pseudo-critical temperature, 40 degree. In Figure 4-5 for all the test cases, the LS model reproduces the measured wall temperatures poorly in contrast to the predictions in the other test cases. The $\mathrm{CH}$ model predicts the wall temperature much better than the LS model but worse than WJ-mAKN. The predictions of the WJ- $m$ AKN model are in good agreement with the data except the sharp peak of the wall temperature near the entrance of heating. Kurganov and Kaptilnyi [1992] found that when the buoyancy effect is much stronger than the thermal acceleration (as in these test cases where the position of zero shear stress was not the same as the position of zero velocity-gradient), any eddy viscosity model had to incorporate the buoyancy effect and such a model gave better predictions with the experimental data. In the present work, only the WJ-mAKN model can deal with these two positions differently but it is not related directly to the buoyancy effect. So et al. [2002] showed that when the buoyancy force term was incorporated into an explicit algebraic stress model for buoyant homogeneous flow, the prediction improved at high Richardson numbers.

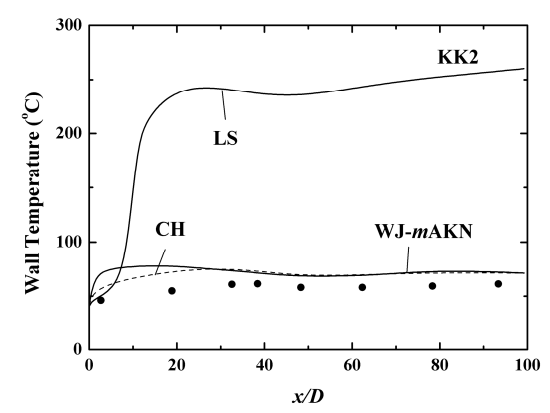

(a)

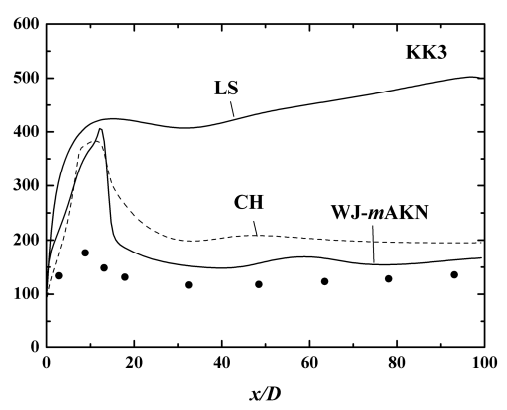

(b)

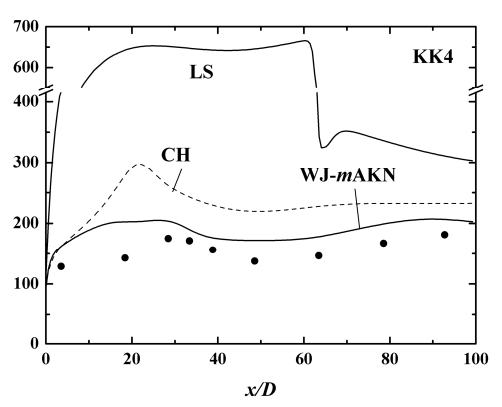

(c)

Fig. 4-5. Wall temperature distributions for supercritical $\mathrm{CO}_{2}$ flows compared with the data of Kurganov \& Kaptilnyi [1992] at p $0.76 \approx \mathrm{MPa}$.

4-2-3-3. Test of DSM closure using FLUENT commercial code. The prediction capability of twoequation model and the DSM closure are tested using commercial code, FLUENT. In FLUENT there are the standard $k-\varepsilon$ model, realizable $k-\varepsilon$ model, RNG (Renormalized Group) $k-\varepsilon$ model and $k-\omega$ model in two-equation models and the LRR (Launder, Reece \& Rodi) model in their Reynolds stress model. For the eddy diffusivity of energy, a constant turbulent Prandtl number of 0.9 was used (it is the inevitable option in FLUENT) and this approach has limitations in the flow with buoyancy effects where the heat transport direction is not consistent with that of the temperature gradient. A two-layer method was selected for the wall treatment because no low-Reynolds number $k-\varepsilon$ model was available, unfortunately.

The heated pipe flows are simulated using two-equation models and the LRR model in FLUENT with the turbulent Prandtl number at 0.9 (no option in this version of the code). Both are in poor agreement with the data as shown in Figure 4-6 and Figure 4-7. The Nusselt number was overpredicted for both cases. We suspect that this discrepancy was from an inherent problem of FLUENT models at low Reynolds numbers. Overall, we found that it was not adequate to adopt FLUENT to the simulation of these flows.

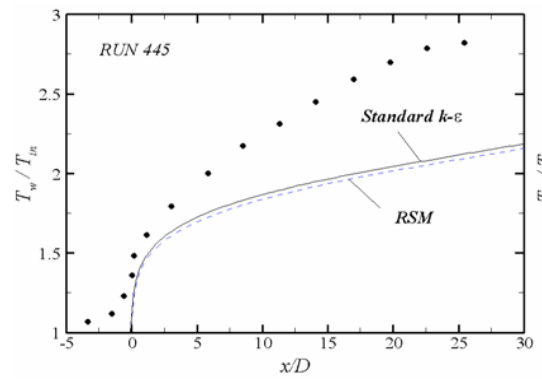

(a)

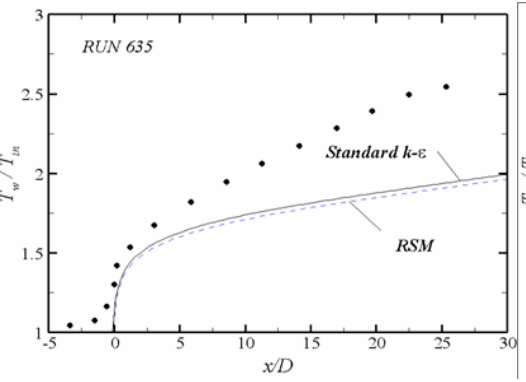

(b)

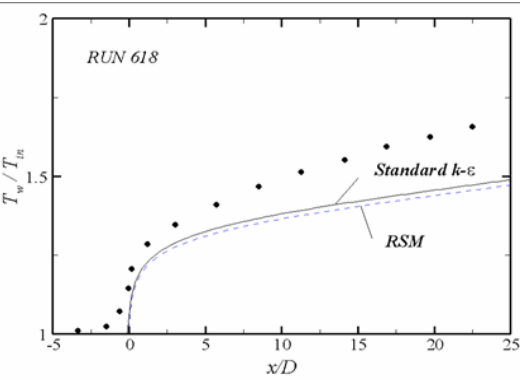

(c)

Fig. 4-6. Comparison of wall temperature distributions for superheated gas (air) flows between FLUENT and the data of Shehata \& McEligot [1998]. 


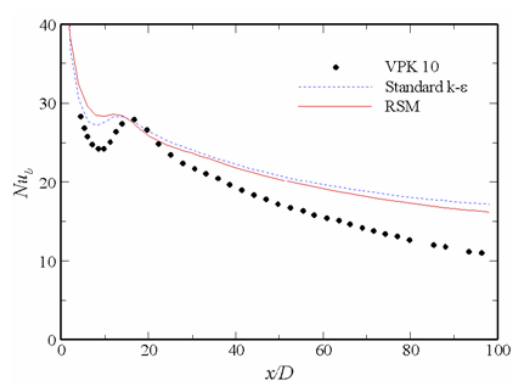

(a)

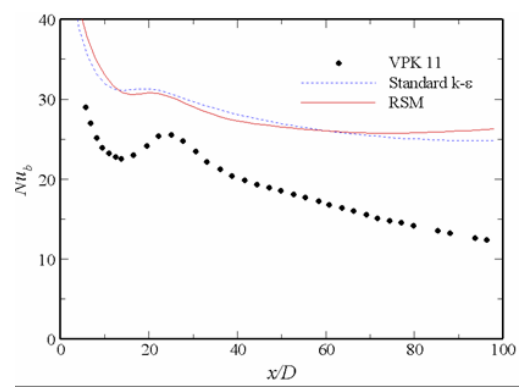

(b)

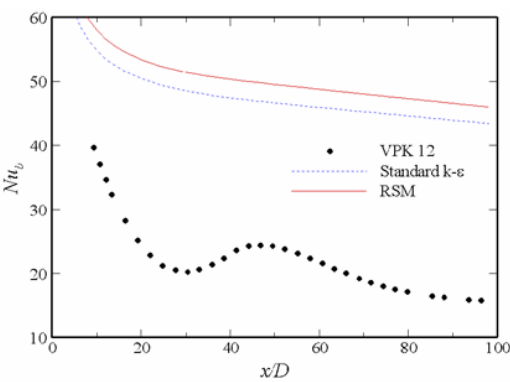

(c)

Fig. 4-7. Comparison of Nusselt number distributions for superheated gas (air) flows between FLUENT models and the data of Vilemas, Poskas and Kaupas [1992].

4-2-4. Modeling Turbulent Heat Flux Near the pseudo-critical temperature for supercritical pressure in Figure 4-8, it is the specific heat capacity that varies most abruptly within a small change of the mean temperature. The density fluctuation is also believed to be significant when the fluid density changes as in supercritical flows. However, for superheated gas flow where the fluid density varies but the specific heat capacity remains nearly constant, Mikielewicz [1994] showed that some two-equation turbulence models predicted the wall temperature distribution reasonably. According to He et al. [2003], the prediction performance of two-equation models for supercritical flows was much poorer than for superheated gas. The main difference between the two situations is the property variation of the specific heat capacity. Further, with the Favre-average technique only one $\operatorname{term}\left(\overline{\rho u_{j}^{\prime}}\right)$ that includes the effect of density fluctuation appears in the kinetic energy equation and it is modeled as $-\beta \overline{\rho u_{j}^{\prime} T^{\prime}}$, referred to as the buoyancy production of turbulence kinetic energy. As mentioned in the previous section, there apparently is no special advantage to employ the combination of EASM and EAHFM in the simple geometry.

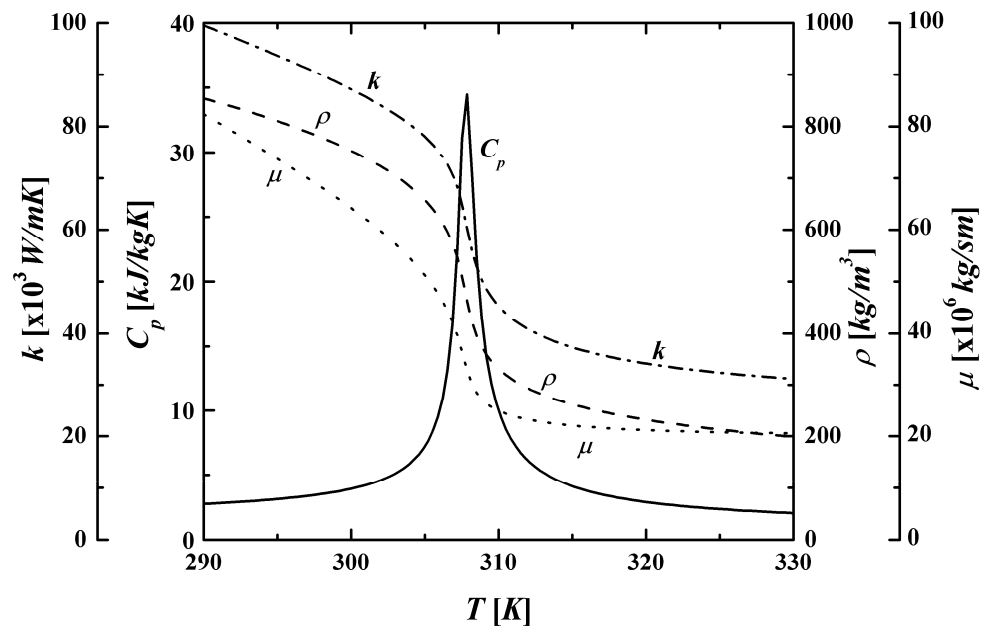

Fig. 4-8. Property variations of $\mathrm{CO}_{2}$ near the pseudo-critical temperature at $8 \mathrm{MPa}$.

In the present work, prior to considering the effect of density variation, we first examine whether the gradient-diffusion model of turbulent heat flux is valid for property-variation. In the conventional model, the turbulent heat flux term, $-\overline{\rho u_{j}^{\prime} h^{\prime}}$, is obtained by multiplying the model of $-\overline{\rho u_{j}^{\prime} T^{\prime}}$ by the specific heat capacity. This treatment is from the constant property assumption. For the case of flow where the specific heat capacity can vary significantly, their conventional approach for a turbulent heat-flux model may not be adequate. 


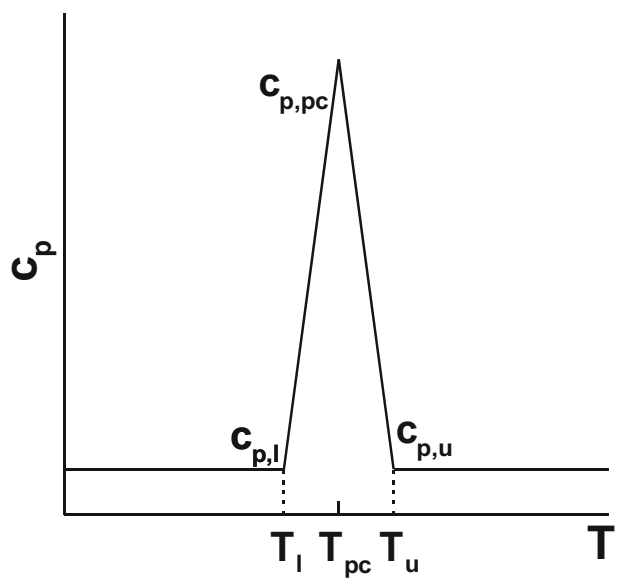

Fig. 4-9. Simplified approximation of specific heat capacity near the pseudo-critical temperature.

To model the turbulent heat-flux, we simplify the specific heat capacity variation as shown in Figure 4-9. For $\bar{T}<T_{l}$ and $\bar{T}>T_{u}$, since $c_{p}$ is nearly constant, we have

$$
h \approx c_{p} T
$$

By decomposing Equation (4-47) into mean and the fluctuation components, we get

$$
h^{\prime}=\bar{c}_{p} T^{\prime}+c_{p}^{\prime} \bar{T}+c_{p}^{\prime} T^{\prime}-\overline{c_{p}^{\prime} T^{\prime}}
$$

On neglecting the higher-order terms, we may write

$$
h^{\prime} \approx \bar{c}_{p} T^{\prime}+c_{p}^{\prime} \bar{T}
$$

For $T_{l}<\bar{T}<T_{p c}$, as $c_{p}$ increases linearly, we have

$$
h-h_{l}=\int_{T_{l}}^{T} c_{p} d T=\frac{1}{2}\left(T-T_{l}\right)\left(c_{p, l}+c_{p}\right)
$$

In a similar manner, Equation (4-49) can be written as

$$
h^{\prime} \approx \frac{1}{2} T^{\prime}\left(\bar{c}_{p, l}+\bar{c}_{p}\right)+\frac{1}{2} c_{p}^{\prime}\left(\bar{T}-\bar{T}_{l}\right)
$$

For $T_{p c}<\bar{T}<T_{u}$, we get

$$
h-h_{p c}=\int_{T_{p c}}^{T} c_{p} d T \approx \frac{1}{2}\left(T-T_{p c}\right)\left(c_{p, p c}+c_{p}\right) \& h^{\prime} \approx \frac{1}{2} T^{\prime}\left(\bar{c}_{p, p c}+\bar{c}_{p}\right)+\frac{1}{2} c_{p}^{\prime}\left(\bar{T}-\bar{T}_{p c}\right)
$$

In summary, the enthalpy fluctuation can be approximated as

$$
h^{\prime} \approx \frac{1}{2} T^{\prime}\left(\bar{c}_{p, k}+\bar{c}_{p}\right)+\frac{1}{2} c_{p}^{\prime}\left(\bar{T}-\bar{T}_{k}\right)
$$


with

$$
\bar{T}_{k}=\left\{\begin{array}{ll}
\bar{T} & \text { if } \bar{T}_{l}>\bar{T} \text { or } \bar{T}_{u}<\bar{T} \\
\bar{T}_{l} & \text { if } \bar{T}_{l}<\bar{T}<\bar{T}_{p c} \\
\bar{T}_{p c} & \text { if } \bar{T}_{p c}<\bar{T}<\bar{T}_{u}
\end{array} \text { and } \bar{c}_{p, k}= \begin{cases}\bar{c}_{p} & \text { if } \bar{T}_{l}>\bar{T} \text { or } \bar{T}_{u}<\bar{T} \\
\bar{c}_{p, l} & \text { if } \bar{T}_{l}<\bar{T}<\bar{T}_{p c} \\
\bar{c}_{p, p c} & \text { if } \bar{T}_{p c}<\bar{T}<\bar{T}_{u}\end{cases}\right.
$$

Multiplying Eq. (4-52) by the density and the velocity fluctuation and taking the Reynolds average gives

$$
\begin{aligned}
\overline{\rho u_{j}^{\prime} h^{\prime}} & =\frac{1}{2} \overline{\rho u_{j}^{\prime} T^{\prime}}\left(\bar{c}_{p, k}+\bar{c}_{p}\right)+\frac{1}{2} \overline{\rho u_{j}^{\prime} c_{p}^{\prime}}\left(\bar{T}-\bar{T}_{k}\right) \\
& =\frac{1}{2} \bar{c}_{p} \overline{\rho u_{j}^{\prime} T^{\prime}}\left(1+\frac{\bar{c}_{p, k}}{\bar{c}_{p}}\right)\left(1+\frac{\overline{\rho u_{j}^{\prime} c_{p}^{\prime}}}{\overline{\rho u_{j}^{\prime} T^{\prime}}} \frac{\bar{T}}{\bar{c}_{p}} \frac{1-\bar{T}_{k} / \bar{T}}{1+\bar{c}_{p, k} / \bar{c}_{p}}\right)
\end{aligned}
$$

The first term of the right hand side in the above equation, $-\bar{c}_{p} \overline{\rho u_{j}^{\prime} T^{\prime}}$, can be expressed as follows.

$$
-\bar{c}_{p} \overline{\rho u_{j}^{\prime} T^{\prime}}=\bar{c}_{p}\left(\mu_{T} / \operatorname{Pr}_{T}\right) \partial_{j} \tilde{T}=\left(\mu_{T} / \operatorname{Pr}_{T}\right) \partial_{j} \tilde{h}
$$

We have to model the term of $\overline{\rho u_{j}^{\prime} c_{p}^{\prime}} / \overline{\rho u_{j}^{\prime} T^{\prime}}$ in the second bracket. We assume that

$$
\overline{\rho u_{j}^{\prime} c_{p}^{\prime}} / \overline{\rho u_{j}^{\prime} T^{\prime}} \square c_{p}^{\prime} / T^{\prime}=C_{k 1}\left(c_{p, r m s} / T_{r m s}\right)
$$

Here, $C_{k 1}$ is an empirical constant to be determined later. Since the specific heat is a function of temperature, it is generally expressed as the polynomial form of temperature. However, it is difficult to find the magnitude of its fluctuation intuitively. So we expanded the specific heat as

$$
c_{p}(T)=\bar{c}_{p}+\frac{\partial c_{p}}{\partial T}(T-\bar{T})+\frac{1}{2} \frac{\partial^{2} c_{p}}{\partial T^{2}}(T-\bar{T})^{2}+\cdots
$$

From Equation (4-57), we may set

$$
c_{p}^{\prime} \approx \frac{\partial c_{p}}{\partial T} T^{\prime}+\frac{1}{2} \frac{\partial^{2} c_{p}}{\partial T^{2}} T^{\prime 2}
$$

Since $c_{p, r m s}=\sqrt{c_{p}^{\prime 2}}$ and $T_{r m s}=\sqrt{T^{\prime 2}}$, we may write.

$$
c_{p, r m s} / T_{r m s}=\left[\left(\partial c_{p} / \partial T\right)^{2}+C_{k 2} T_{r m s}^{2}\left(\partial^{2} c_{p} / \partial T^{2}\right)^{2}\right]^{0.5}
$$

The second derivative term in the right hand side of Equation (4-58) makes this equation non-zero at the critical point. The quantity $C_{k 2}$ is an empirical constant. We adopt the model of Liuboja and Rodi [1981] for $T_{r m s}$ as

$$
T_{r m s}^{2}=-1.6(k / \varepsilon) \overline{u_{j}^{\prime} T^{\prime}} \partial_{j} T
$$


Finally, the turbulent heat flux of Equation (4-54) can be summarized

$$
-\overline{\rho u_{j}^{\prime} h^{\prime}}=f\left(\mu_{T} / \operatorname{Pr}_{T}\right) \partial_{j} \tilde{h}
$$

with

$$
f=\frac{1}{2}\left(1+\frac{\bar{c}_{p, k}}{\bar{c}_{p}}\right)\left(1+C_{k 1} \frac{c_{p, r m s}}{T_{r m s}} \frac{\bar{T}}{\bar{c}_{p}} \frac{1-\bar{T}_{k} / \bar{T}}{1+\bar{c}_{p, k} / \bar{c}_{p}}\right)
$$

To remove the discontinuity of $T_{k}$ and $c_{p, k}$ in Equation (4-61) and to reduce the computational time, we simplified Equation (4-61) as follows:

$$
f=1+C_{k 1}\left(c_{p, r m s} / T_{r m s}\right)\left(\bar{T} / \bar{c}_{p}\right)
$$

where, $C_{k 1}$ is 0.01 which was determined through numerical optimizations and $C_{k 2}$ in Equation (4-58) was set be unity. The gradient of specific heat with respect to temperature in Equation (4-58) was evaluated at every iteration from a table that was given before the computation to reduce the computational time.

For a turbulence model platform, we selected the model of Launder and Sharma [1974]. The combinations of EASM and EAHFM are not superior to two-equation models in predicting the wall temperature distribution and they can be reduced to a form similar to two-equation models in a simple geometry. So we estiamte that two-equation model is sufficient to take into account of the property variation effect. In addition, from the previous section, we found that the predictions of LS for superheated gas flows were in excellent agreement with the experimental data, but those for supercritical flows were poor. We suspect that these poor predictions are mainly due to the significant property variation. It is the LS model that can show the effect of property variation best.

The test cases are from the direct numerical simulations carried out by Bae, Yoo and Choi [2003]. Carbon dioxide is used as the working fluid at $P=8 \mathrm{MPa}$, slightly above the thermodynamic critical pressure of $P_{c}=7.38 \mathrm{MPa}$. The corresponding pseudo-critical temperature is $307.85 \mathrm{~K}$. We selected three test cases that have the temperature ranges of $T_{b}<T_{p c}<T_{w}$. The inlet conditions are specified in Table 4-5.

Table 4-5: The inlet conditions of the test cases

\begin{tabular}{ccccccc}
\hline Test Case & $\boldsymbol{P}_{\boldsymbol{i n}}(\boldsymbol{M P a})$ & $\boldsymbol{T}_{\boldsymbol{i n}}(\boldsymbol{K})$ & $\boldsymbol{R e}_{\boldsymbol{d}}$ & $\boldsymbol{q}^{+}{ }_{\text {in }}$ & $\boldsymbol{G r}_{\boldsymbol{q}, \boldsymbol{i n}}$ & $\boldsymbol{P r}_{\boldsymbol{i n}}$ \\
\hline SNU1 & 8.0 & 301.1 & 5400 & $1.4 \times 10^{-4}$ & $3.36 \times 10^{6}$ & 3.08 \\
SNU2 & 8.0 & 301.1 & 5400 & $1.4 \times 10^{-4}$ & $2.69 \times 10^{7}$ & 3.08 \\
SNU3 & 8.0 & 301.1 & 5400 & $1.4 \times 10^{-4}$ & $9.06 \times 10^{7}$ & 3.08 \\
\hline
\end{tabular}

The wall temperature distributions are shown in Figure 4-10. We find that the present model improves substantially the prediction performance compared to that of the original Launder-Sharma model. However, the performance deteriorates when the Grashof number is larger (SNU2 case). The mean velocity profiles are plotted at various axial locations in Figure 4-11. We find that the velocity profiles from the present model are in good agreement with the DNS data. The mean temperature profiles are shown in Figure 4-12. The differences among the predictions generally occurs in the region very near the wall where $y / R<0.1$ (approximately $y_{w}^{+}<20 \sim 30$ ). At this location, the mean temperature is around the pseudo-critical temperature. In the vicinity of this region, the molecular activities and the turbulent activities due to the buoyancy force compete each other i.e., They are of the same order-of-magnitude. For the case where the buoyancy force is small (SNU1), the present model performs very 
well as seen in Figure 4-12(a). The developing Reynolds shear stress profiles are shown in Figure 4-13. We see that the predictions by the present model and the Launder-Sharma model deviate considerably from the DNS data, while the Popov model performs better for the case of SNU2. We suspect that this problem is probably due to the lack of buoyancy term in the Reynolds shear stress model of the present model and the Launder-Sharma model.

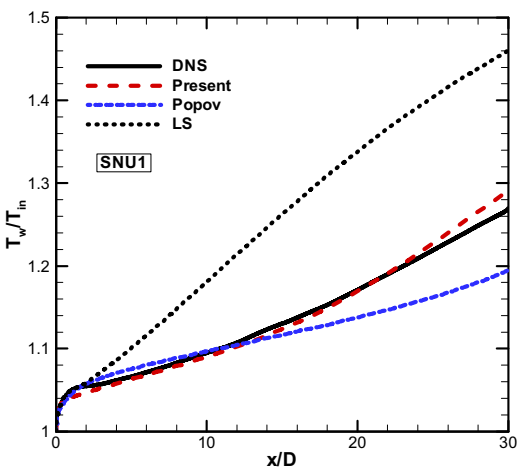

(a)

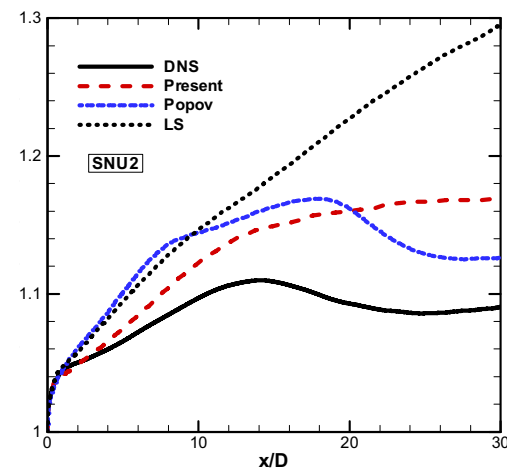

(b)

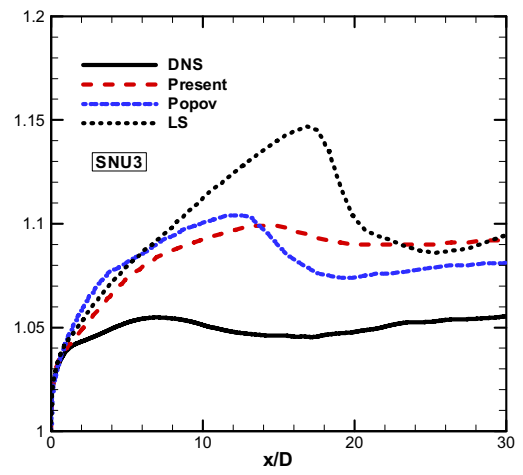

(c)

Fig. 4-10. Wall temperature distributions for supercritical $\mathrm{CO}_{2}$ flows at $8 \mathrm{MPa}$.

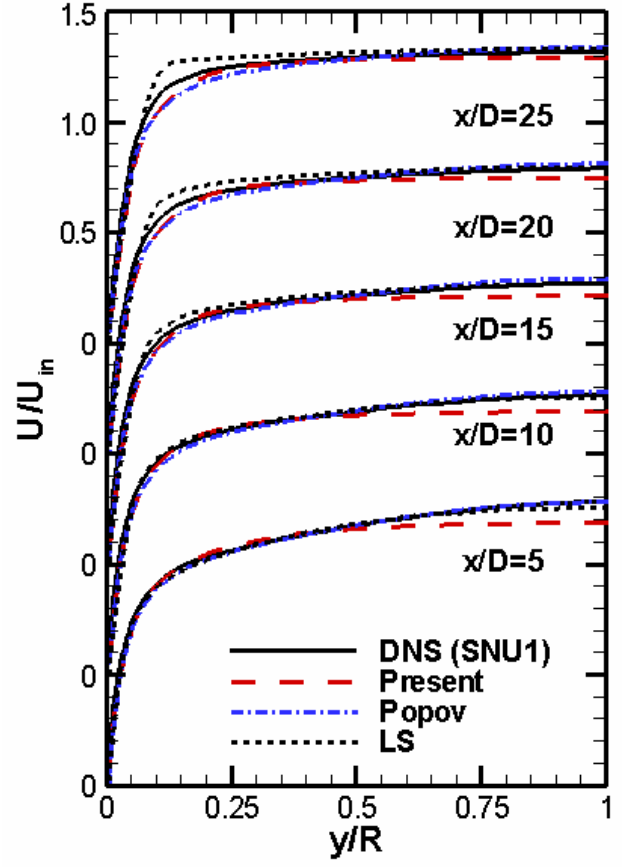

(a)

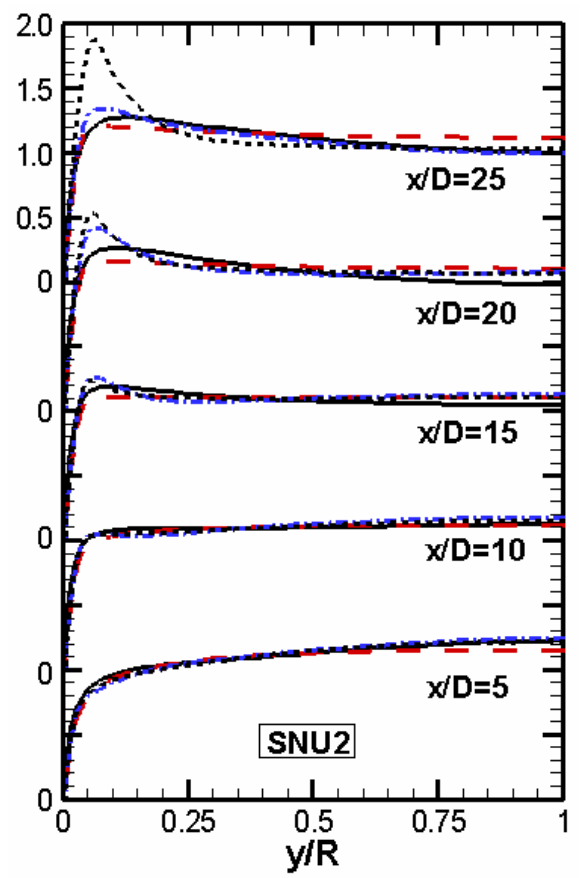

(b)

Fig. 4-11. Mean velocity profiles. 


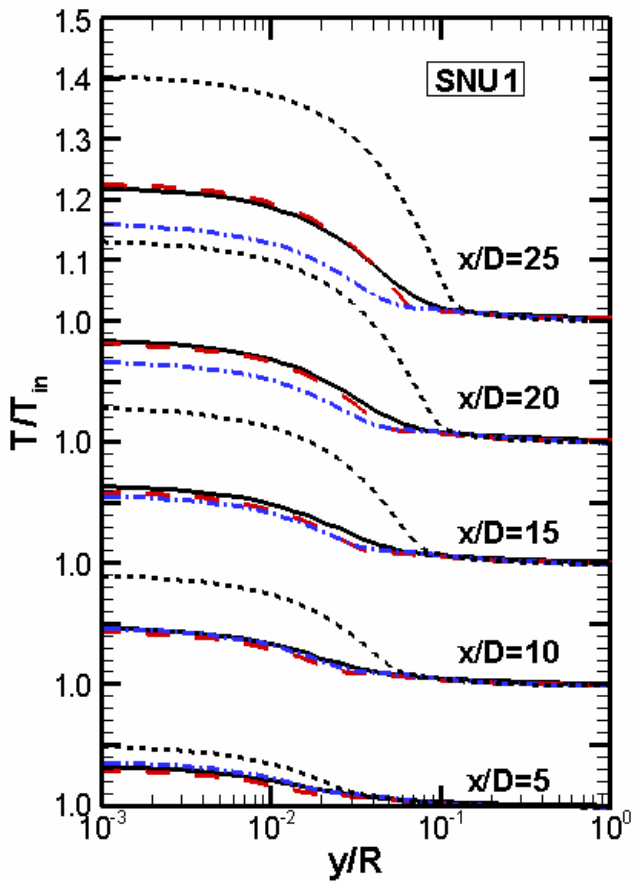

(a)

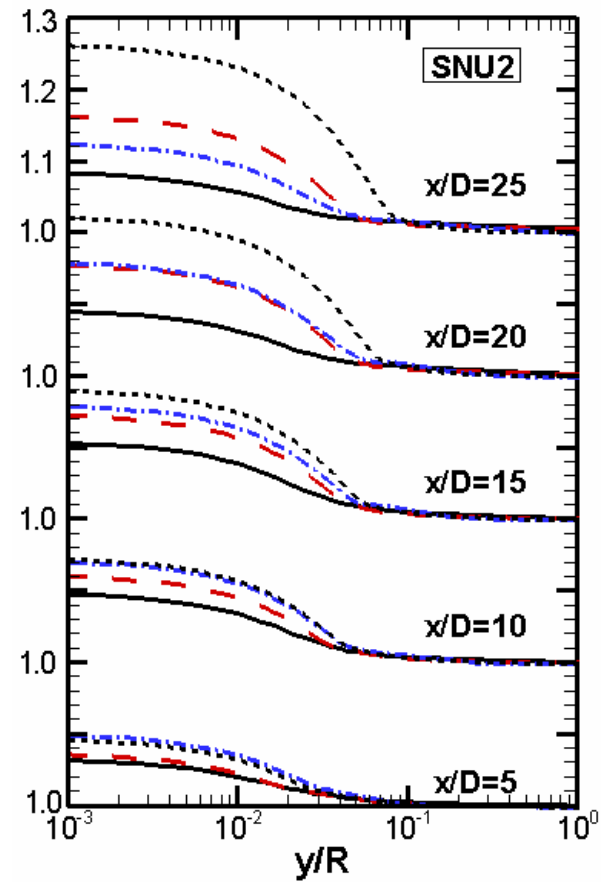

(b)

Fig. 4-12. Mean temperature profiles (legend as in Figure 4-11).

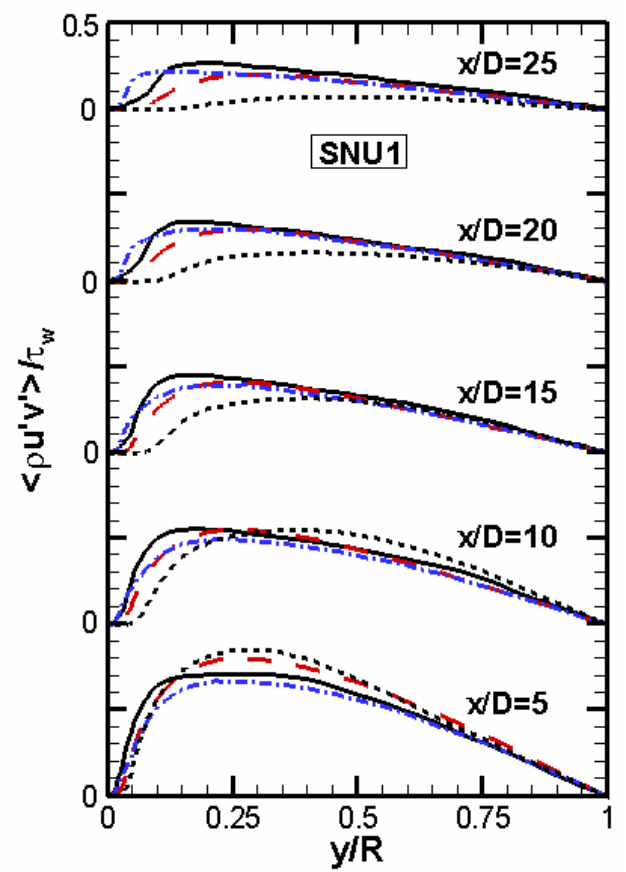

(a)

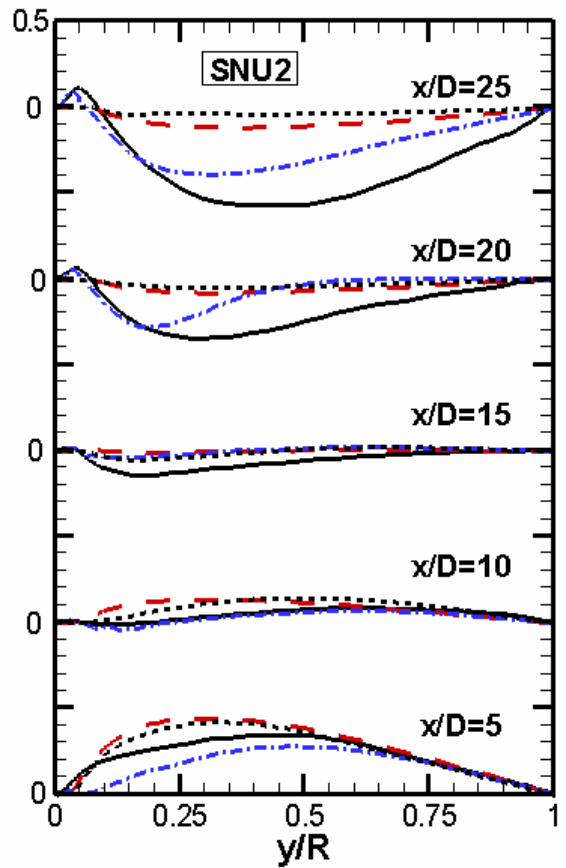

(b)

Fig. 4-13. Reynolds shear stress profiles (legend as in Figure 4-11). 

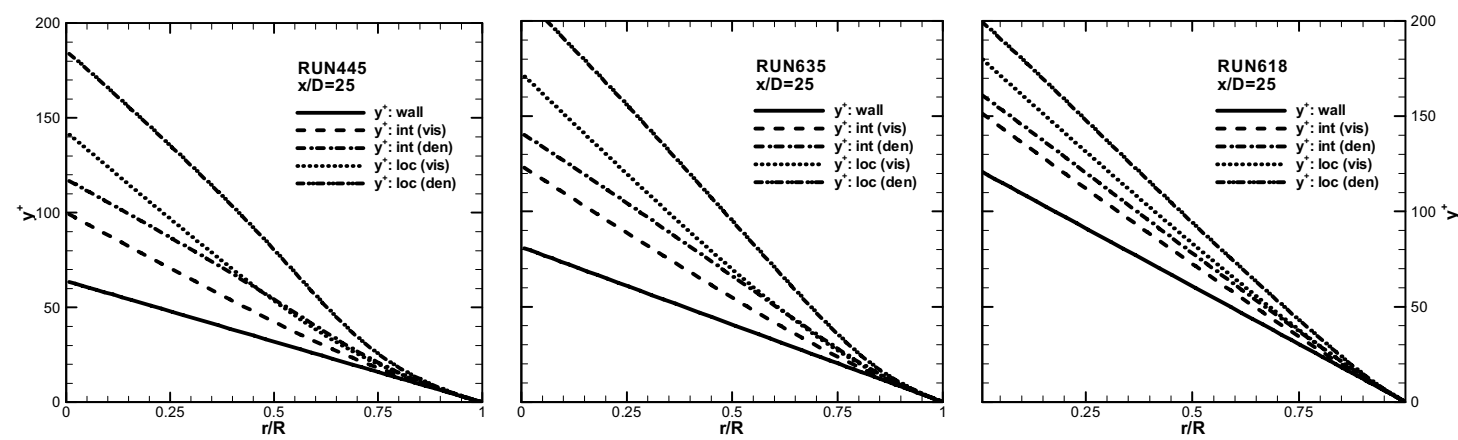

(a) superheated flow
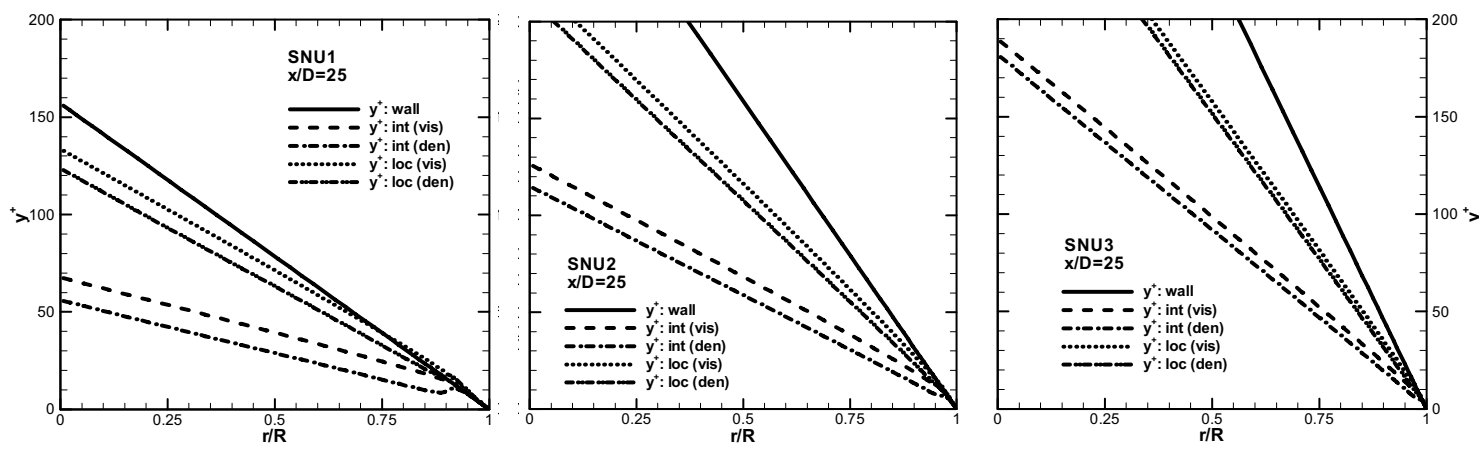

(b) supercritical flow

Fig. 4-14. Comparison of five different definitions of wall distance coordinate calculated from DNS data for superheated gas (air) flow and supercritical carbon dioxide flows

4-2-5. Effects of wall distance definition For two-equation turbulence models, the effect of the wall distance coordinate has not yet been reported systematically for variable property flow. In this report we look into this issue. First of all, we derive possible various definitions of wall distance coordinate. According to each definition, we investigate the results of a turbulence model for property-variation flows: superheated gas flows and supercritical pressure $\mathrm{CO}_{2}$ flows near the pseudo-critical temperature in a heated vertical pipe with definitions as follows

$$
\begin{gathered}
y_{\text {wall }}^{+}=\frac{\rho_{w} \sqrt{\tau_{w} / \rho_{w}}}{\mu_{w}} y: \text { wall-property unit } \\
y_{\text {int,den }}^{+}=\int_{0}^{y} \frac{\rho \sqrt{\tau_{w} / \rho}}{\mu} d y: \text { integral-property unit } \\
y_{\text {loc,den }}^{+}=\frac{\rho \sqrt{\tau_{w} / \rho}}{\mu} y: \text { local-property unit }
\end{gathered}
$$

4-2-5-1. Superheated flows From the DNS data of Bae, Yoo and Choi [2003] for superheated gas flows with significant property variation, comparisons are made between those calculated by three definitions of wall distance coordinate and the DNS data in figure 4-14. Figure 4-15 shows the predicted kin friction variations of all the calculations: three test cases with three turbulence models using three different definitions of wall distance unit. 
Away from the thermal entry $(\mathrm{x} / \mathrm{D}=0)$, the calculated skin friction factor variations are seen to converge. Although all predictions are not in good agreement with the DNS data, the results have a common tendency that the calculated skin friction becomes highest when using the 'local-property' $y^{+}$of Equation (4-65), smallest when using the 'wallproperty' $y^{+}$of Equation (4-63) and is intermediate when using the 'integral-property' $y^{+}$of Equation (4-64). For Nusselt number in Fig. 4-16, we have similar observations but the difference between predictions by the three wall distance units are smaller than that in skin friction.

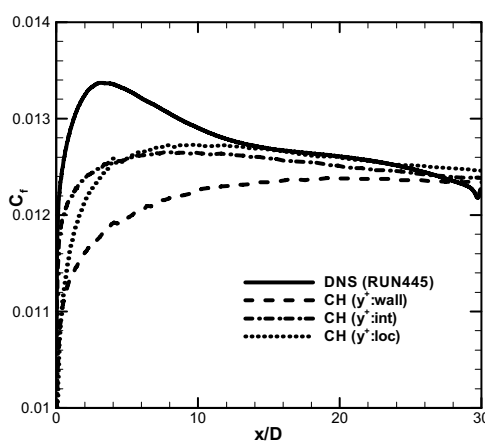

(a)

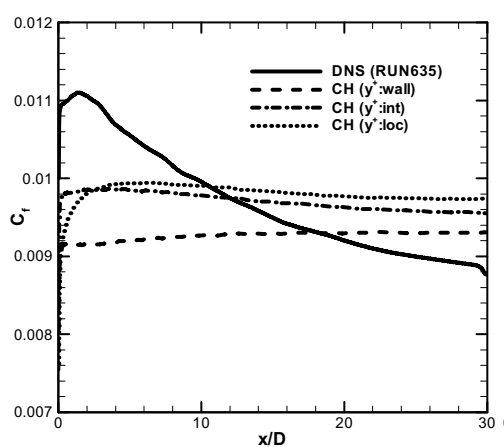

(d)

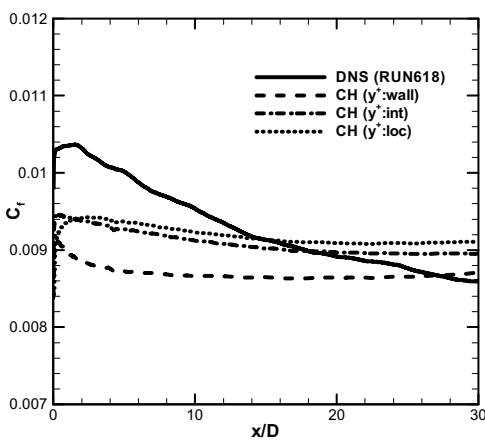

(g)

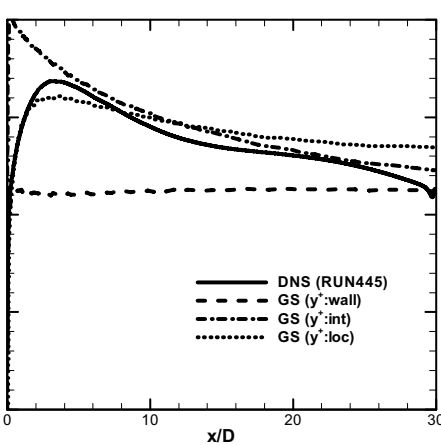

(b)

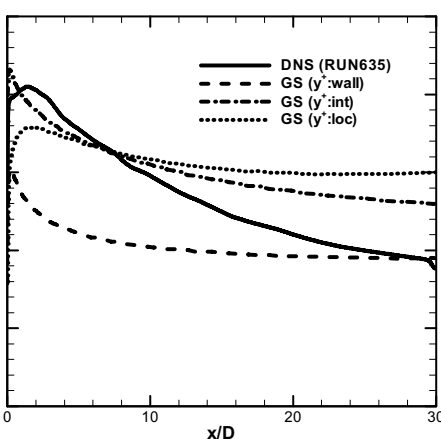

(e)

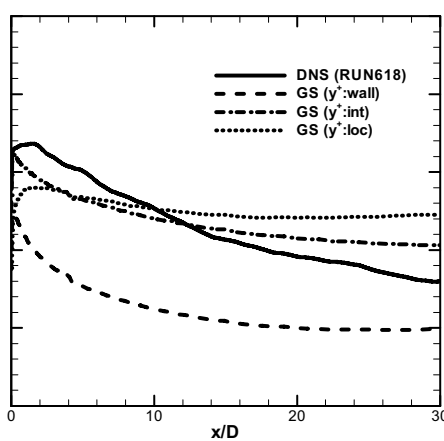

(h)

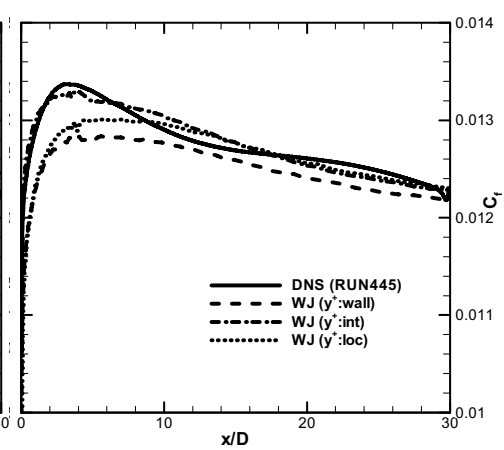

(c)

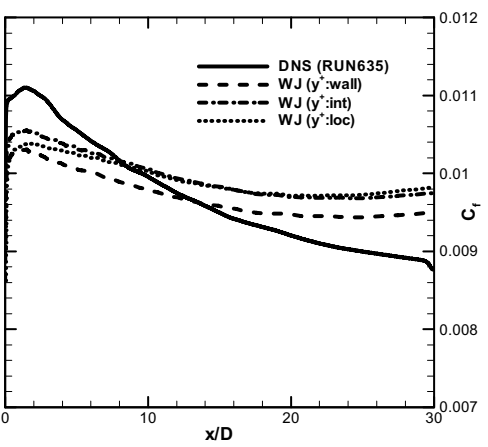

(f)

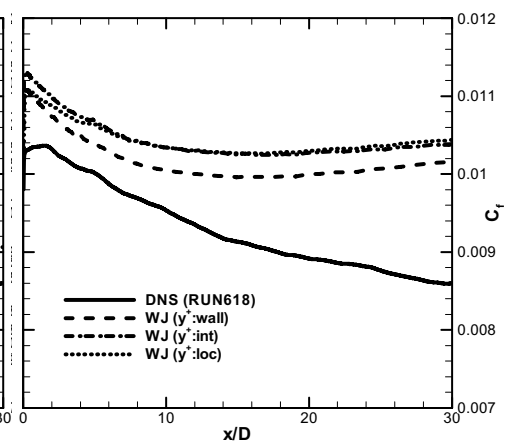

(i)

Fig. 4-15. Variations of skin friction coefficient with wall coordinate definitions for superheated gas (air) flows. 


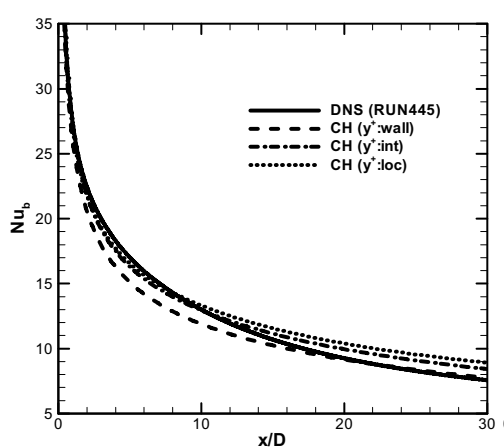

(a)

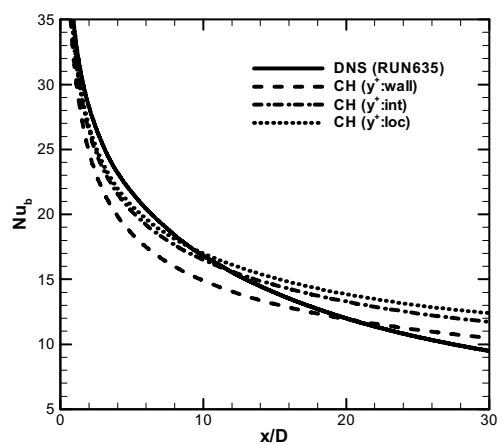

(d)

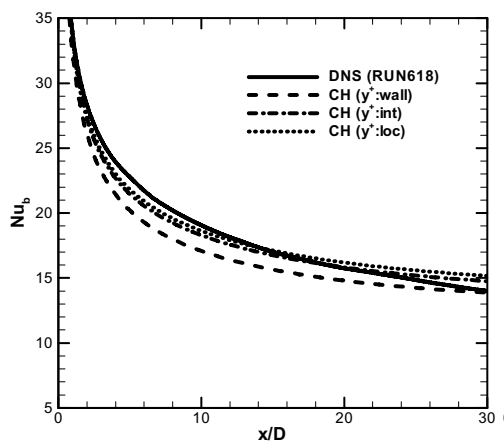

(g)

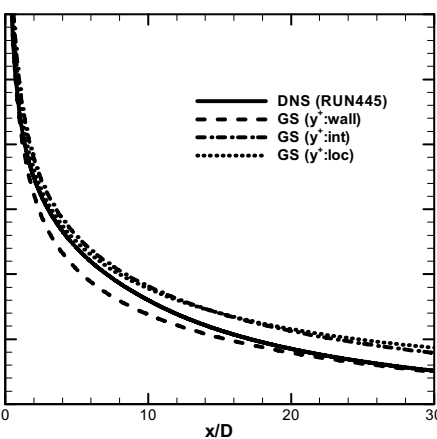

(b)

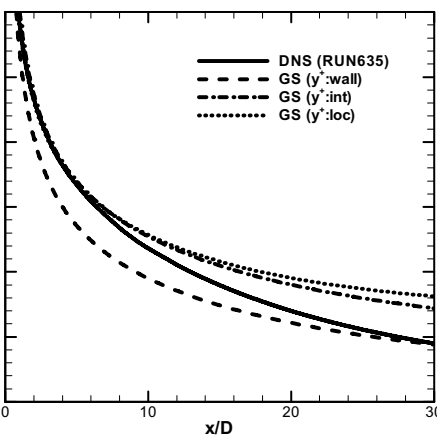

(e)

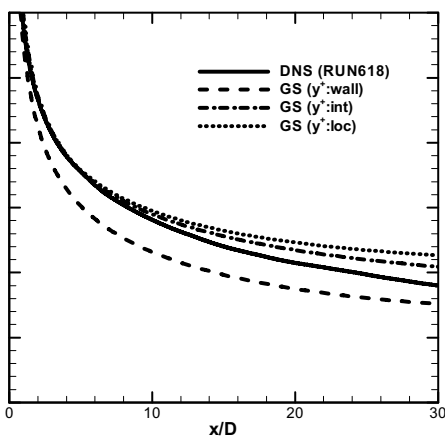

(h)

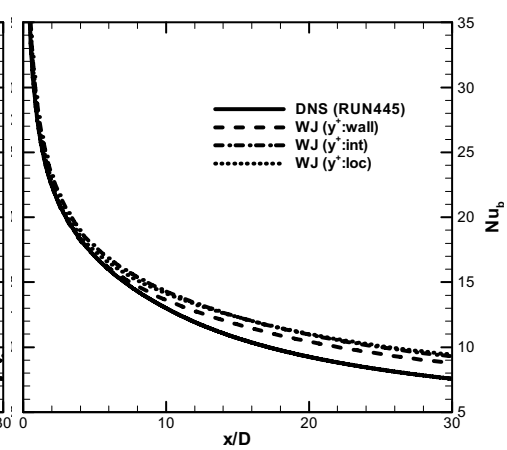

(c)

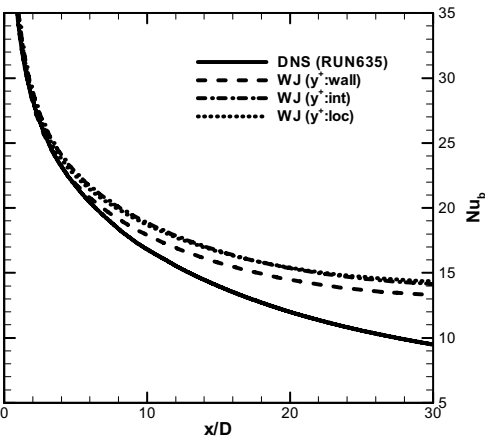

(f)

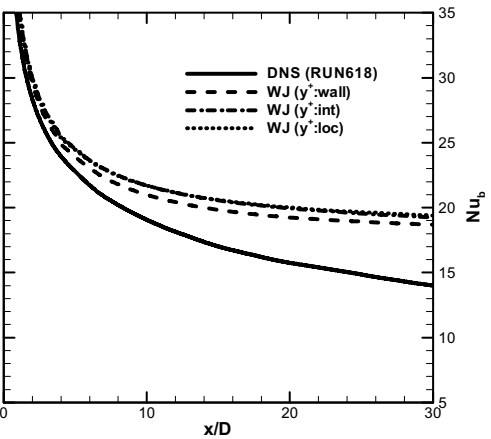

(i)

Fig. 4-16. Variations of Nusselt number distributions with wall coordinate definitions for superheated gas flows.

In Figures 4-15 and 16, all the predictions with the three different definitions of wall distance coordinate converged within about twenty per cent among three predictions at $\mathrm{x} / \mathrm{D}=30$. The results using three definitions with the $\mathrm{CH}$ and WJ models are within ten per cent in both skin friction factor and Nusselt number. With GS model they are within ten per cent. The turbulence models employing the 'wall-property' definition of Equation (4-63) gave the most satisfactory results.

To examine further the effects of the wall distance coordinate, we choose one computational case that shows distinct differences the predictions of skin friction and Nusselt number. Figure 4-17 shows the calculated mean velocity, turbulent kinetic energy, and wall temperature profiles with DNS data at $\mathrm{x} / \mathrm{D}=25$ for RUN618 by the GS turbulence model. Here $U_{w}^{+}=U / u_{\tau}$ and $y_{w}^{+}=y \sqrt{\tau_{w} \rho_{w}} / \mu_{w}$. As mentioned in a previous section, at a certain 
position for superheated gas flow, the 'local-property' $y^{+}$of Equation (4-65) is the greatest, 'integral-property' $y^{+}$ of Equation (4-64) is the second, and the 'wall-property' $y^{+}$Equation (4-63) of is the smallest. In contrast, for the wall unit to reach a threshold, the 'wall-property' $y^{+}$gives the longest distance from the wall and the 'localproperty' $y^{+}$gives the shortest. For example, this distance corresponds to the effective damping region near the wall. In these flows, employing 'wall-property' $y^{+}$in a turbulence model means that the effective damping region is set to be large near the wall, so that the turbulent activity near the wall is damped out in wider region than the others. This reduction of turbulent kinetic energy reduces the momentum transfer and the heat transfer. Eventually, the mean velocity is lower and the mean temperature is higher near the wall. This effect is seen in Figure 4-17.

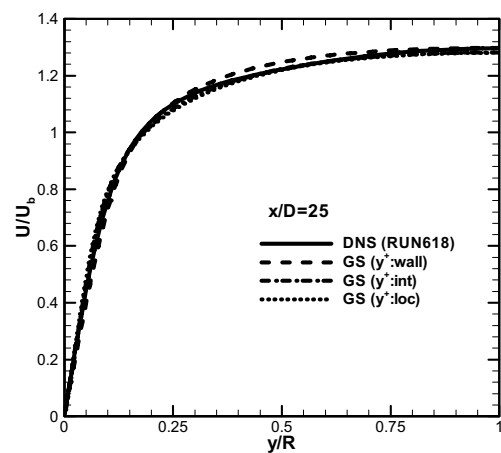

(a)

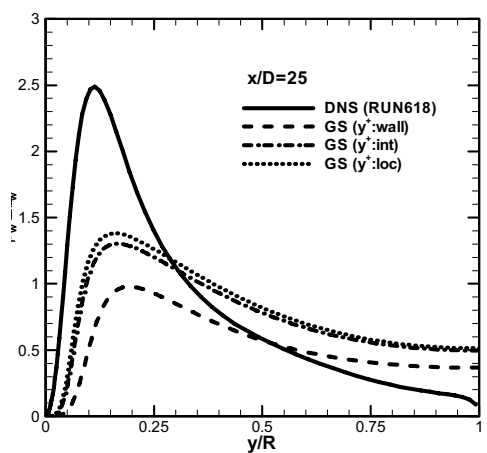

(b)

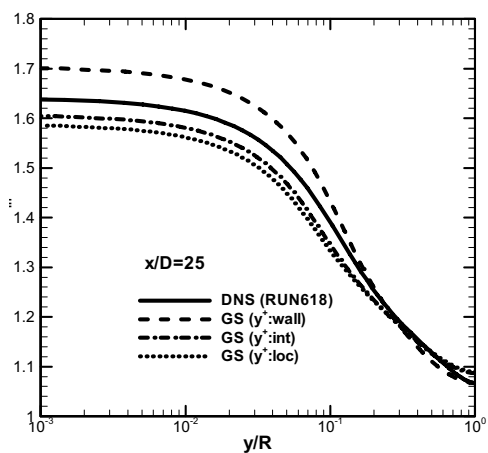

(c)

Fig. 4-17. Predicted mean velocity, turbulent kinetic energy and mean temperature profiles at $\mathrm{x} / \mathrm{D}=25$ for superheated gas flows with GS turbulence model;

4-2-5-2. Supercritical flows Figure 4-18 compares the calculated skin friction factor variations for supercritical $\mathrm{CO}_{2}$ flows with DNS data. The differences between predictions by the three definitions of wall distance coordinate for each turbulence model are smaller than those for superheated gas flows. Comparing Nusselt number in Figure 4-19 with skin friction, one sees that for the SNU3 case the smaller errors in skin friction give larger errors in Nusselt number than for the other cases. This opbservation is an interesting feature but is beyond our scope to study further.

In Figures 4-18 and 19, although predictions are not all in good agreement with DNS data, there are less than about $10 \%$ differences in the predicted skin friction factors and Nusselt numbers at $\mathrm{x} / \mathrm{D}=30$ for the three different wall distance coordinates. Lee and Howell [1998] showed that there were less than three per cent differences in the predictions of heat transfer coefficients with a mixing length model and that the 'local-property' $y^{+}$of Equation (465) gave the highest value, similar with the present results. 


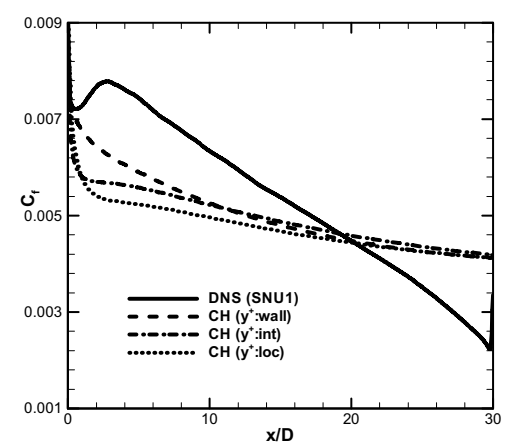

(a)

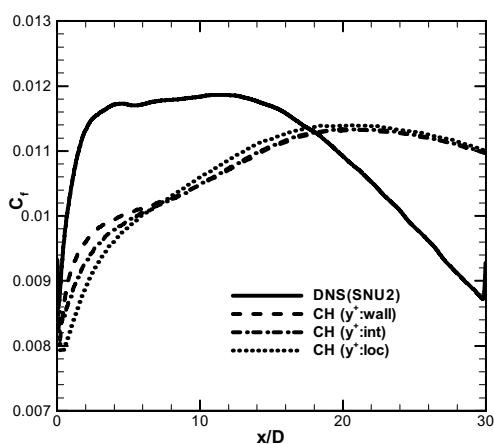

(d)

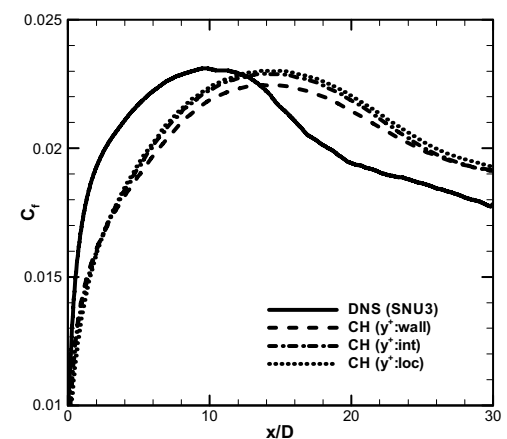

(g)

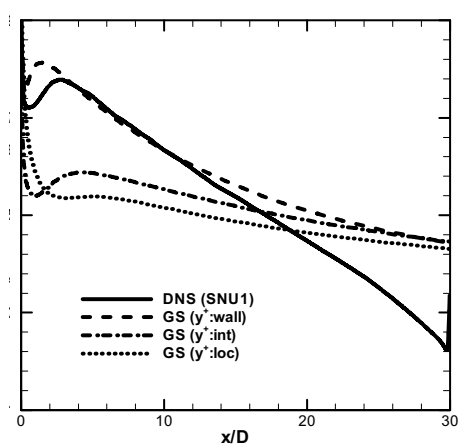

(b)

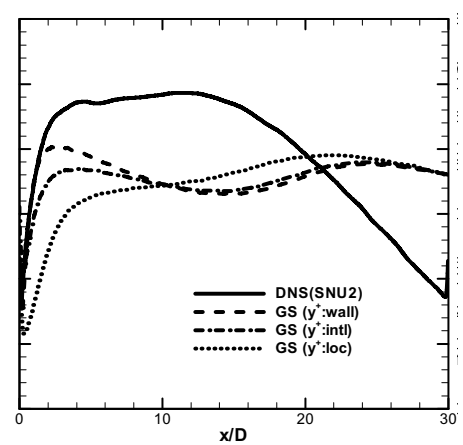

(e)

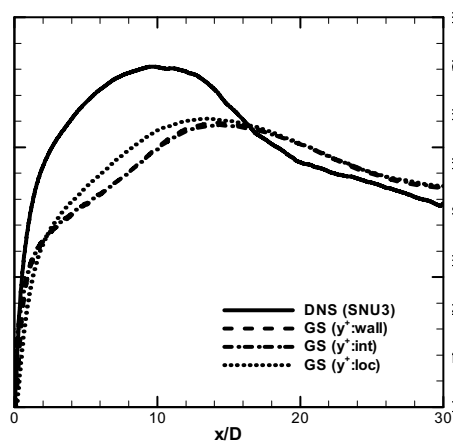

(h)

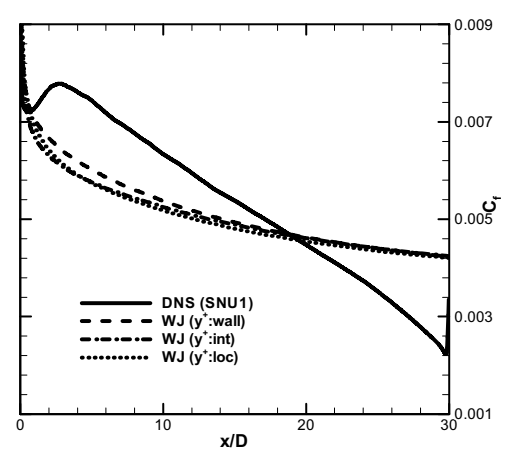

(c)

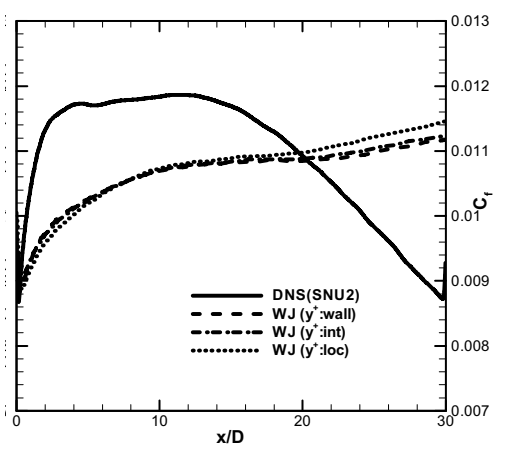

(f)

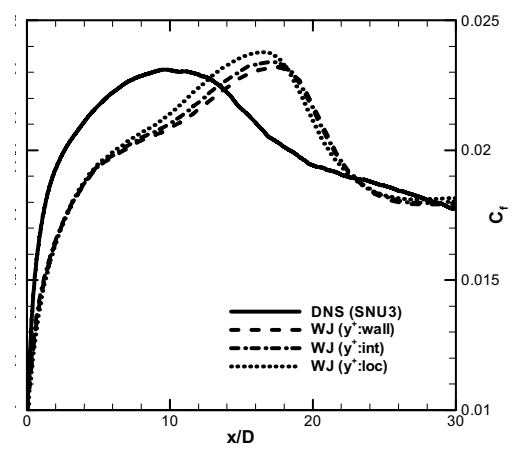

(i)

Fig. 4-18. Variations of skin friction factor with wall coordinate definitions for supercritical $\mathrm{CO}_{2}$ flows. 


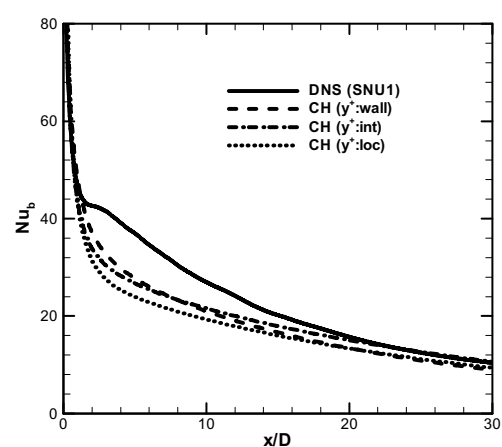

(a)

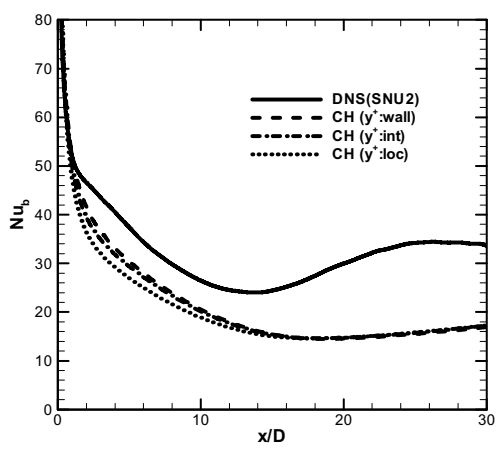

(d)

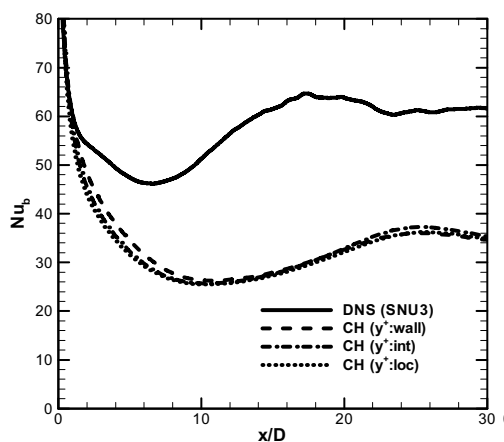

(g)

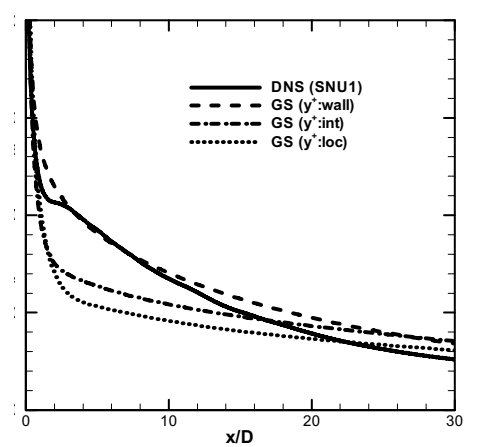

(b)

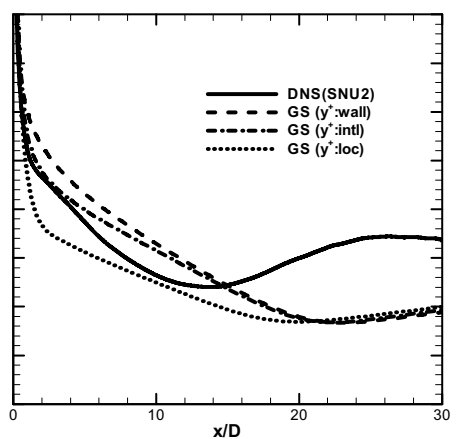

(e)

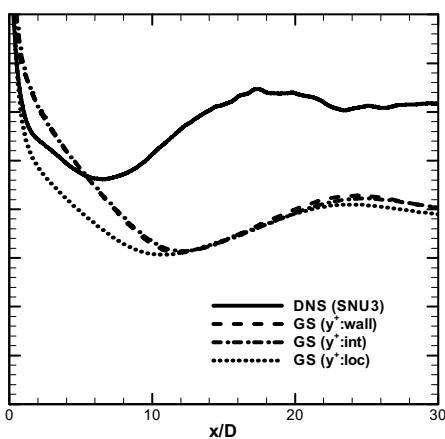

(h)

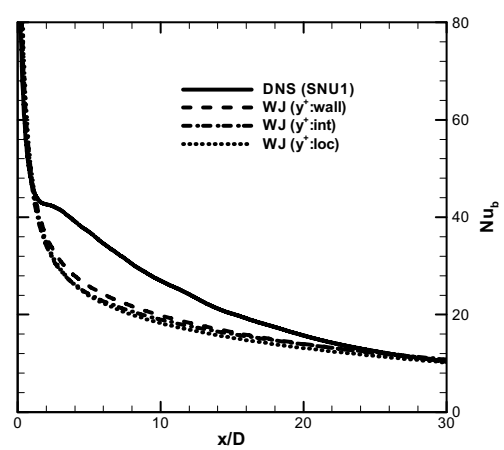

(c)

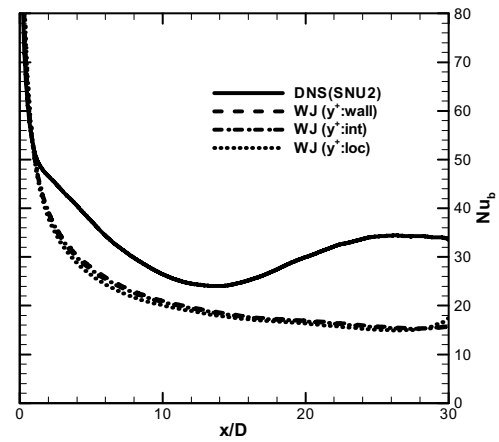

(f)

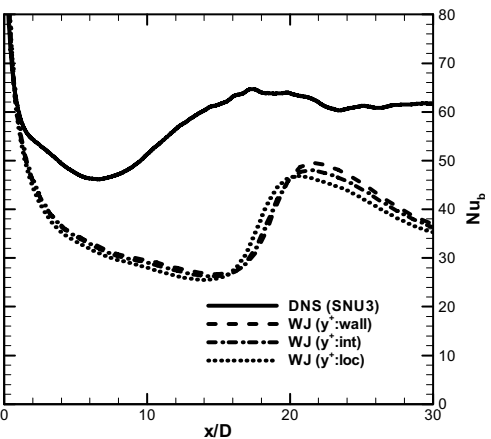

(i)

Fig. 4-19. Variations of Nusselt number distributions with wall coordinate definitions for supercritical $\mathrm{CO}_{2}$ flows.

For supercritical flows, in contrast to the case of superheated gas flows, the 'local-property' $y^{+}$of Equation (4-65) is the smallest at a local position, as shown earlier in Figure 4-14. Therefore, the local-property definition is expected to widen the effective damping region, so that turbulent activity can be hardly transferred the near wall region and the mean velocity component near the wall is decreased. Based on the pervious analysis of the wallproperty $y^{+}$for superheated gas flows, this effect would result in reducedskin friction and Nusselt number. However, this result is not seen in the calculated skin friction variations for SNU2 and SNU3 cases from Figure 418.

To check in this point, we select two different axial positions, $\mathrm{x} / \mathrm{D}=5$ and $\mathrm{x} / \mathrm{D}=25$, from Figure 4-18(e) and we plot the mean velocity, the turbulent kinetic energy, and the mean temperature profiles in Figures 4-20 and 21, 
respectively. At $x / D=5$ in Figures 4-20, there is no significant difference in velocity profiles, comparing the analysis of the wall distance effect of 'local-property' $y^{+}$for superheated gas flows. At $\mathrm{x} / \mathrm{D}=25$, however, with the wall unit of wall-property definition, the mean velocity and the turbulence kinetic energy near the wall are reduced in Figure 4-20, respectively.

The key observation lies in the mean velocity profile. With an M-type mean velocity profile in Figure 4$21(a)$, the mean velocity component near the wall $(y / R<0.1)$ is largely controlled by the buoyancy force. When the buoyancy force is strong, the near wall velocity component increases. This effect means that the buoyancy force poses an obstacle to momentum transfer to the near wall region. Since the 'local-property' $y^{+}$damps the turbulent activity near the wall according to the analysis, it forces the effective buoyancy force to be larger. That situation affects the mean velocity and leads to the augmented skin friction. The turbulent kinetic energy with the localproperty definition of wall distance coordinate in Figures 4-21(b) is increased by the high mean shear rate in the core region due to the reduction of momentum transfer.

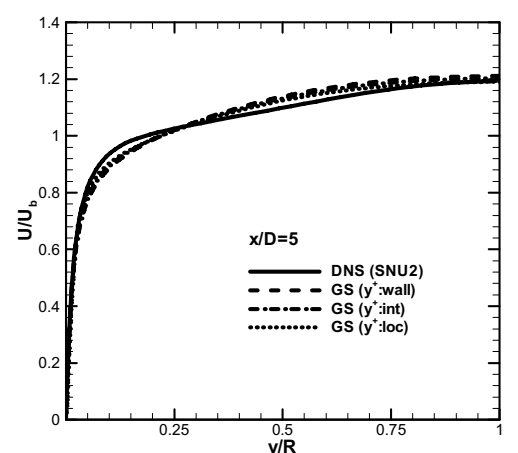

(a)

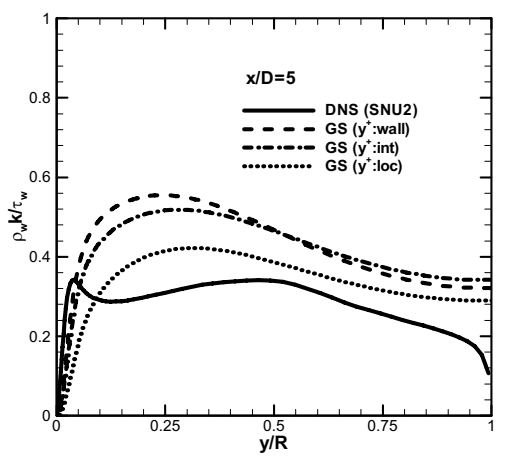

(b)

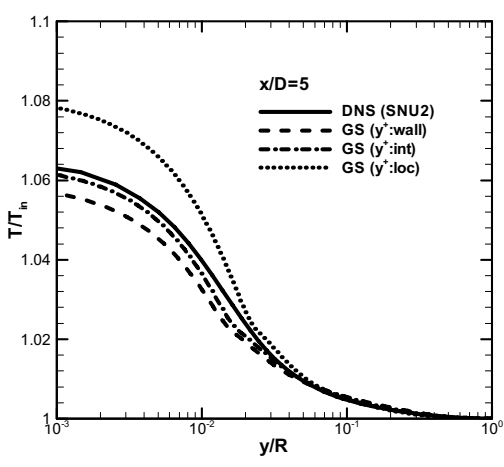

(c)

Fig. 4-21. Mean velocity, turbulent kinetic energy and mean temperature profiles at $\mathrm{x} / \mathrm{D}=5$ for supercritical carbon dioxide $\mathrm{CO}_{2}$ flows with GS turbulence model.

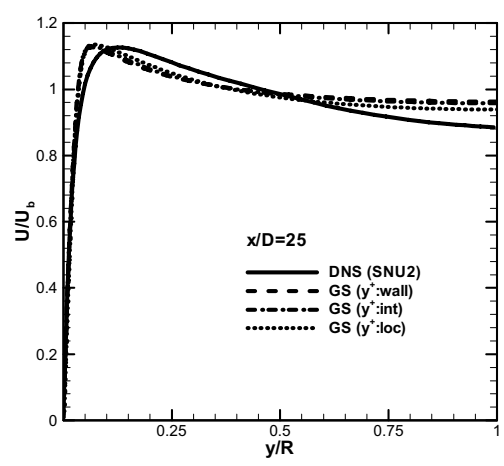

(a)

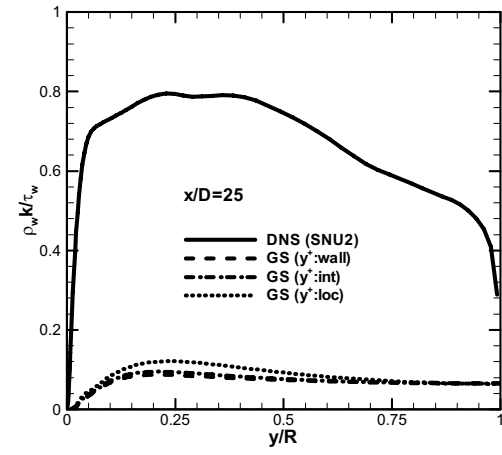

(b)

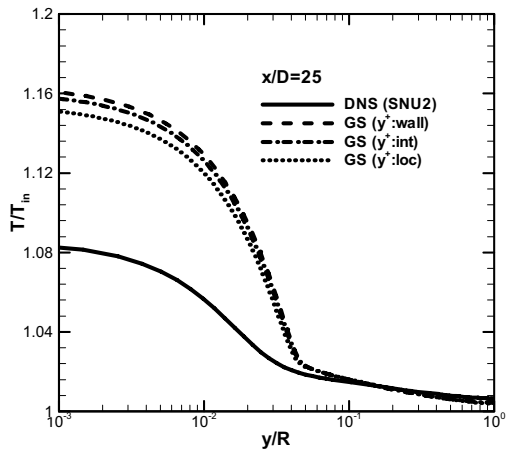

(c)

Fig. 4-21. Mean velocity, turbulent kinetic energy and mean temperature profiles at $\mathrm{x} / \mathrm{D}=25$ for supercritical $\mathrm{CO}_{2}$ flows with GS turbulence model.

4-2-6. EASM considering the buoyancy effects For a strongly buoyant flow, the buoyancy force affects the Reynolds shear stress. In this section, we first consider effects of the buoyancy force in the model of the Reynolds stress tensor. Fortunately, So et al. [2002] already have obtained the Reynolds stress tensor with the buoyancy effect by deriving an explicit algebraic stress model for buoyant flow. It was developed based on the approach of Gatski and Speziale [1993] as follows 


$$
\begin{aligned}
\overline{u_{i}^{\prime} u_{j}^{\prime}}= & \frac{2}{3} k \delta_{i j}-2 v_{t}\left[S_{i j}+\alpha_{4} \lambda\left(S_{i k} W_{k j}+S_{j k} W_{k i}\right)-\alpha_{5} \lambda\left(S_{i k} S_{k j}-\frac{1}{3} S_{n m} S_{n m} \delta_{i j}\right)\right] \\
& +2 C_{b}(k / \varepsilon)\left[f_{i j}^{\prime}+\alpha_{4} \lambda\left(f_{i k}^{\prime} W_{k j}+f_{j k}^{\prime} W_{k i}\right)-\alpha_{6} \lambda^{2} G\left(S_{i k} S_{k j}-\frac{1}{3} S_{n m} S_{n m} \delta_{i j}\right)\right]
\end{aligned}
$$

where,

$$
v_{t}=C_{\mu}^{*} f_{b}\left(k^{2} / \varepsilon\right) \text { and } C_{b}=\beta_{1} /\left(1+\xi^{2}\right)
$$

with

$$
f_{b}=1+\beta_{4} C_{b}(G / \varepsilon)\left(1-\eta_{8}-2 \eta_{14}+\xi^{2}\right)
$$

The invariants are defined by

$$
\eta_{8}=\beta_{2}(\lambda / G)\left(f_{i j}^{\prime} S_{i j}\right) \text { and } \eta_{14}=\beta_{3}\left(\lambda^{2} / G\right)\left(W_{i k} S_{k j} f_{j i}^{\prime}\right)
$$

where $\lambda=k / \varepsilon$ and $f_{i j}^{\prime}$ is given by

$$
f_{i j}^{\prime}=G_{i j}-\delta_{i j}^{(2 d)} G
$$

and the $\beta$ - coefficients are

$$
\beta_{1}=\left(1-C_{6}\right)(g / 2), \beta_{2}=\left(2-C_{3}\right)(g / 2), \text { and } \beta_{3}=\left(2-C_{3}\right)\left(2-C_{4}\right)\left(g^{2} / 4\right)
$$

We simplify the above equation for channel flow. After several mathematical manipulations, we obtain the following equation:

$$
-\overline{u v}=v_{t}^{*} \frac{\partial U}{\partial y}-\frac{2 C_{b 2}^{*}}{1+\eta} \frac{k}{\varepsilon} g \beta \overline{v \theta}
$$

where,

$$
\begin{gathered}
v_{t}^{*}=C_{\mu}^{*} f_{b} \frac{k^{2}}{\varepsilon}=v_{t}^{T M} f_{b} \\
f_{b}=1-C_{b 1}^{*} \frac{1+\eta+\xi}{1+\eta} \frac{G}{\varepsilon}, \eta=2 a_{2}^{2} g^{\prime 2} \lambda^{2}\left(2 S_{12}^{2}\right), \xi=\frac{a_{3}^{2}}{\left(3 a_{2}-a_{3}\right)} g^{\prime} \lambda\left(2 G_{12} S_{12} / G\right) \\
G=0.5 G_{11}, G_{11}=g \beta \bar{u} \theta, G_{12}=g \beta \overline{v \theta}
\end{gathered}
$$

and the coefficients are

$$
a_{2}=0.82, a_{3}=0.38, C_{b 1}^{*}=0.29 g^{\prime}, C_{b 2}^{*}=0.7 g^{\prime}
$$


Here, $g$ represents the gravitational acceleration and $g^{\prime}$ is defined by

$$
(1 / g)=\left(C_{1}^{*} / 2+1\right)(\tilde{P} / \varepsilon)+C_{1} / 2-1+(G / \varepsilon)
$$

Since the $\tilde{P} / \varepsilon$ term that is included in the explicit algebraic stress model includes the Reynolds stress, the model is not fully explicit. To obtain a fully explicit form of the Reynolds stress tensor, there are two ways. One is simple. We set $g^{\prime}$ to be constant, e.g.,

$$
g^{\prime} \approx 0.4
$$

The other way is to solve a complex equation to obtain the value of $\bar{P} / \varepsilon$. In these two methods we have to choose one. But it is not necessary to employ the "fully explicit" model. Then we have three choices; the last one is to use the original form (which is implicit form).

In general, most turbulence model for the buoyancy term have been developed from modeling $\overline{\rho^{\prime} u^{\prime}}$ and $\beta \overline{u^{\prime} T^{\prime}}$ via the Boussinesq approximation, thereby assuming that the density variation is negligible. However, it is not proper to adopt this modeling to supercritical flow where density variation is large. So it seems that modeling of a mass transport term considering the buoyancy effect will be necessary. Also Favre (or mass weighted-) averaging is adopted in compressible Navier-Stokes governing equations which consider the density variation, while in incompressible N-S equations Reynolds-averaging is conducted and then extra terms need to be modeled. For the numerical simulations of supercritical flow, using the Favre-averaging operator in the governing equations is reasonable. However, the buoyancy term is not present explicitly when this operator is used and further study about this issue is required.

4-3. Concluding remarks In the present work, the predictive capabilities of turbulence models are assessed in the superheated gas flow and supercritical flows with significant property variations in a vertical pipe. Twoequations turbulence models and the RSM model in FLUENT show discrepancies in the prediction of temperature, Nusselt number, etc. So various combinations of EASM and EAHFM models are adopted for simulation of these flows with property variation. The results depend on the models selected and it is difficult to choose a 'best' model. A model accounting for the variation of specific heat capacity is developed and is found to perform better for supercritical flow. However, in the case when the buoyancy effect is not negligible, this model's predictions are not yet satisfactory. The effects of wall distance definition are also examined. For supercritical flow predictions, further investigation is needed to deal effectively with density variations beyond the Boussinesq approximation in a turbulence modeling perspective. 
Task 5. Complex flow measurements - experiments for assessment $\quad$ D. M. McEligot, K. G. Condie, G. E. McCreery, H. M. Mcllroy and R.J. Pink, INL, and Prof. B. L. Smith, Utah State University

The goals of the INL experimental portion of the study have been to answer scientific needs identified in the Introduction and to guide code development and assess code capabilities for treating the generic forced convection problems in Generation IV reactor systems. The INL Matched-Index-of-Refraction (MIR) flow system was employed with particle image velocimetry (PIV); it provides means for velocity measurements for the portion of the study dwelling on forced convection in complex reactor geometries. For the representative geometry, the experimental model provides a generic simulation of flow along a closely-packed array of fuel rods separated by periodic grid spacers as in several SCWR concepts. The objectives are to complete PIV measurements, to analyze the fluid physics phenomena and to archive these benchmark data for use by CFD analysts.

INL Matched-Index-of-Refraction flow system Unheated MIR (Matched-Index-of-Refraction) experiments are first steps when the geometry is complicated. One does not want to use a computational technique which will not even handle constant properties properly. The MIR experiment simulates flow features of fuel channels with periodic grid spacers positioning idealized fuel elements. Useful optical flow measurements in this complex configuration would be impractical without refractive-index-matching.

The benefit of the MIR technique is that it permits optical measurements to determine flow characteristics in passages and around objects to be obtained without locating a disturbing transducer in the flow field and without distortion of the optical paths. With a transparent model of different refractive index than the working fluid, the optical rays can be refracted in such a manner that measurements are either impossible (e.g., cannot "see" the desired location) or require extensive, difficult calibrations. Thompson, Bouchery and Lowney [1995] demonstrated this situation conceptually when laser Doppler velocimetry is applied to a rod bundle; with refractive-index-matching the measurement and determination are relatively straight forward while without matching the beams may not cross to form the measurement control volume at the desired focal length, if they cross at all. A demonstration of the benefits of refractive-index-matching is shown in Figure 5-1 with a test model for an idealized ribbed annulus [McCreery et al., 2003] in our recent NERI project. The MIR technique is not new itself; Corino and Brodkey [1969] employed it to measure turbulence structure in a circular tube earlier. Recent applications of the technique include, but are not limited to, those of Durst, Jovanovic and Sender [1993], Parker and Merati [1996], Cui and Adrian [1997] and Becker et al. [2002].

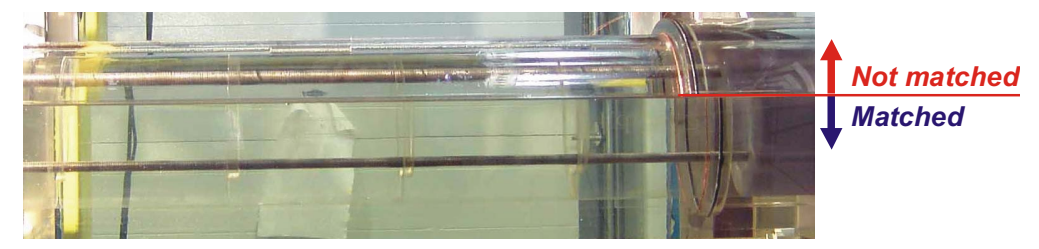

Fig. 5-1. A demonstration of the benefits of refractive-index-matching with a transparent model having curved interfaces (flow through a horizontal ribbed annulus of $164 \mathrm{~mm}$ diameter). Horizontal structural rods are steel and therefore opaque; the right end is plastic which is not matched.

The innovative advantage of the INL system is its large size, leading to improved spatial and temporal resolution compared to others. To date most other experiments with index matching have been small, with characteristic lengths of the order of five $\mathrm{cm}$ or less. In contrast, the INL MIR test section has a cross section of about $60 \mathrm{~cm} \times 60 \mathrm{~cm}$ and is about two meters long, allowing the use of models of substantial size (Figure 5-2). Since the system volume is over 3000 gallons, a light mineral oil ("baby oil without perfume") was selected as the working fluid due to environmental and safety considerations; its refractive index matches that of some quartz. With the fluid temperature controlled, the quartz model can barely be seen at some wave lengths in the visible spectrum (e.g., Figure 5-1); indices are typically matched for the blue or green beams of an argon-ion laser or the green PIV light sheet from a Nd:YAG laser. The design flow rate can give Reynolds numbers up to about $10^{5}$ based on the cross section of the test section. The refractive index of the fluid is maintained at the desired value by a parallel temperature control system which maintains a constant temperature in the test section to within $0.1 \mathrm{C}$ or 
better. In measurements in an experiment on transition induced by a square rib, meaningful velocity and turbulence data were obtained as close to the surface as $\mathrm{y}^{+}=\left(\mathrm{y}_{\tau} / \mathrm{v}\right) \approx 0.1$ and less [Becker et al., 2002].

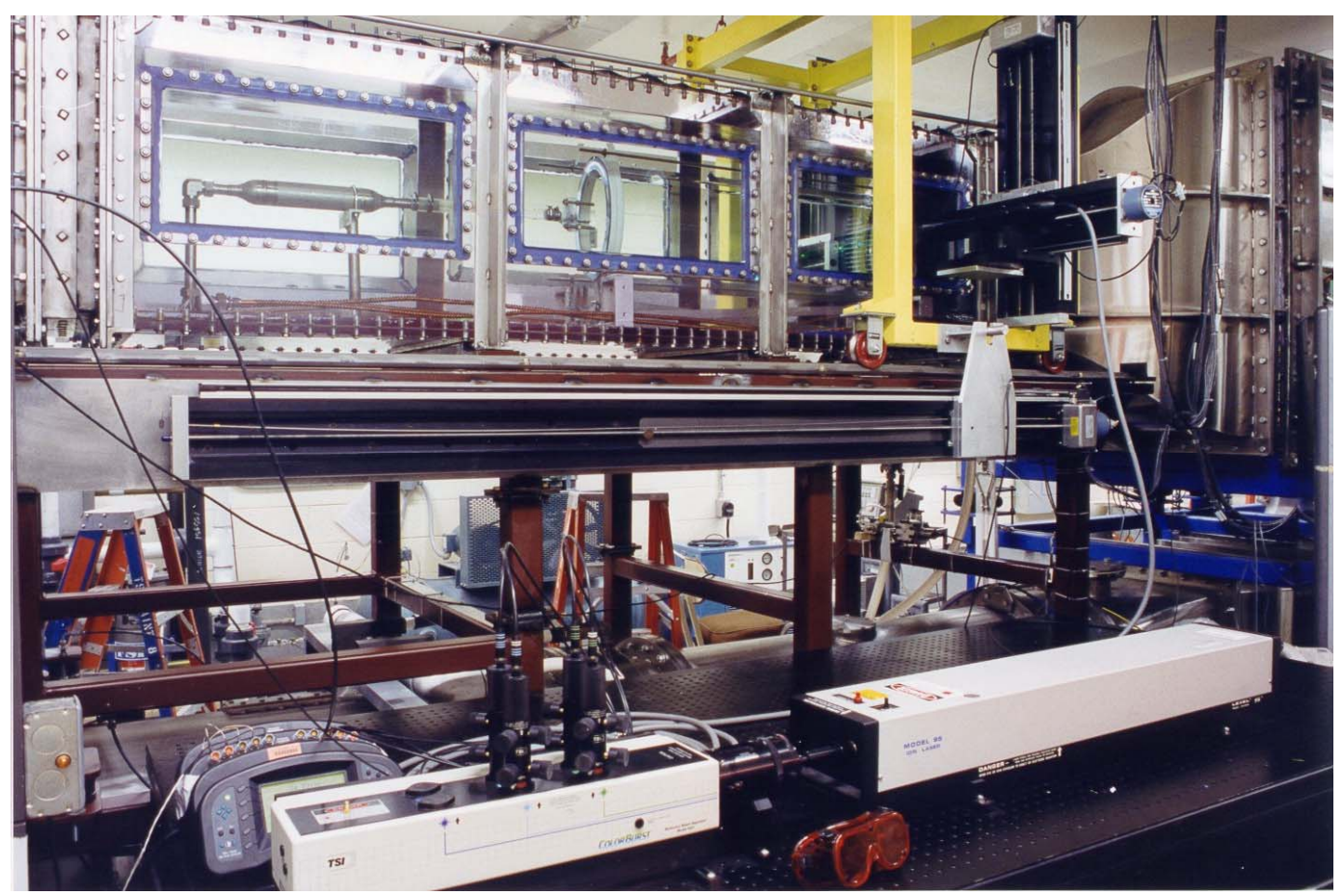

Fig. 5-2. The World's largest Matched-Index-of-Refraction flow system using laser Doppler velocimetry to study fluid physics phenomena in an idealized SNF storage canister for a DoE EM Science project [Stoots et al., 2001; McCreery et al., 2002].

Since the refractive index varies with temperature, it is necessary to conduct MIR experiments with a constant, uniform temperature in both any internal flow model and the external flow around it in the main test section. To maintain the flow through the model at the required temperature, a "Model (Auxiliary) Flow Loop Temperature Control System" has been developed to be comparable in operation to the successful temperature control system for the main flow loop. It includes a heat exchanger to remove the energy introduced by the model loop pump, an electrical heater and its control for fine adjustment and a pump and flowmeter unit. The temperatures of both the main loop and the model loop are controlled through a LabView program.

Flow field and turbulence measurements are conducted by optical techniques, primarily particle image velocimetry (PIV). Visualization of mean flows and instantaneous measurement of three velocity components are available via the PIV system. The PIV system from LaVision, Inc. (Ypsilanti, Mich. 48197) is used for twocomponent or three-component instantaneous and mean velocity component measurements in a two-dimensional laser light sheet. A vertical light sheet is provided by a dual cavity pulsed Nd:YAG laser system from Big Sky Laser Technologies. (Bozeman, Mont. 59715) -- with maximum powers of $120 \mathrm{~mJ} /$ pulse at $532 \mathrm{~nm}$ at a maximum pulse rate of $15 \mathrm{~Hz}$-- with adjustable light sheet optics. Minimum light sheet thickness is about one mm. The lasers and optics are mounted on a two-directional traversing system under the MIR test section so that the light sheet can be positioned by a stepping motor to within $0.013 \mathrm{~mm}$ in the cross stream direction; its position is measured to within about $0.01 \mathrm{~mm}$ with a linear caliper. One or two cameras view the light sheet from mountings on our threedirectional LDV (laser Doppler velocimeter) traversing mechanism, giving 2-D and 3-D capability, respectively. The cameras are the LaVision "Imager Pro Plus 2M" model with 1648 x 1214 pixel resolution and 14-bit dynamic range with two-stage Peltier cooling and the possibility of better than $100 \mathrm{nsec}$ interframe time. Lenses with fifty $\mathrm{mm}$ and $105 \mathrm{~mm}$ focal lengths are available for "large" and "small" fields of view, respectively. The system computer is a dual processor PC with $\mathrm{P} 4$ processors having speeds of $3.06 \mathrm{GHz}$ and storage of $1 \mathrm{~GB}$ RAM, $120 \mathrm{~GB}$ and $300 \mathrm{~GB}$ internal hard drives, read/write DVD, $1.44 \mathrm{MB}$ floppy drive, an external $300 \mathrm{~GB}$ Maxtor OneTouch II hard drive and an external Sony AIT-1 tape drive for 91 GB tapes. A Windows XP operating system is employed. 
Software has both PIV and PTV (particle tracking velocimetry) algorithms and provides instantaneous and mean velocity components, turbulence statistics and velocity gradients across the image from the two-dimensional light sheet.

Experiment $\quad$ For the experiment, typical rod bundle arrays with grid spacers were reviewed for actual and proposed LWR and SCR designs. An aim of the experiment design was to select a model which induces generic flow features of typical LWR / SCR designs (e.g., periodicity, grid spacers, small pitch-to-diameter ratios, etc.) as well as ease in construction and in modeling (for the code developers). SCR concepts examined included a thermal reactor and a fast breeder reactor of Prof. Oka and colleagues at U. Tokyo, a thermal design by INEEL and Forschungszentrum Karlsruhe and a CANDU version from Canada (e.g., Figure E-1). One sees a variety of configurations for fuel assemblies; they may be in a triangular/hexagonal pattern or rectangular one or random circular distribution, but inevitably they are closely-spaced. Some examples of typical grid spacers are shown in Figure I-2; here too, there is no single generic geometry. Spacing is tight so probe instrumentation should not be used since probes would disturb the flow to be measured. Optical techniques are desired. However, useful optical flow measurements in this realistic configuration could be impractical without refractive-index-matching.

The choice of the scaled rod diameter is primarily dictated by the desire to achieve Reynolds numbers sufficiently high to avoid low-Reynolds-number effects and to approach asymptotic turbulent behavior with the existing model flow pump and auxiliary loop plumbing. However, there are other important design considerations, which include the optical quality of the design, the ease and cost of construction, the fluid temperature rise within the bundle due to viscous heating, the correspondence of the design to a prototype design, stimulation of typical subchannel mixing and the ease of modeling of the design in CFD codes.

For this idealized experiment the desired features include

- Generic features of SCWR fuel geometry

- $\quad$ Close spacing

- $\quad$ Periodic grid spacers

- $\quad$ Turbulent -----> laminar $(1000<\mathrm{ReD}, \mathrm{h}<\sim 10,000)$

- Measurements of $\mathrm{u}, \mathrm{v}, \mathrm{w}$ as functions of $\mathrm{x}, \mathrm{y}, \mathrm{z}$ in representative cell -------> U, u, uv, etc.

- Symmetry or transverse periodicity (to ease computations for comparisons)

- Geometric ratios in generic idealization comparable to typical SCWR applications.

Proposed inlet flow rates gave Reynolds numbers in the range of about 2000 to 10,000 based on hydraulic diameter; the latter value should be high enough to avoid significant low-Reynolds-number effects and be close to asymptotic high-Reynolds-number behavior. Experience shows streamwise periodic flow typically is reached after two to three repeating geometric cells [Berner, Durst and McEligot, 1984; Habib, Durst and McEligot, 1984]; likewise, one or two cells are needed at the end to avoid upstream influence from the exit. Therefore, a minimum of five cells between grid spacers is needed to establish a representative cell for measurements with its flow pattern corresponding to the periodic conditions of the application. For this idealized experiment, six are used.

For the model we selected a two-rod configuration which includes some flow aspects of the thermal SCWR concepts suggested by Oka et al. [2002], Forschungszentrum Karlsruhe [Cheng and Schulenberg, 2002] and INEEL [MacDonald et al., 2002]. These concepts have apparently not progressed to the point of including designs of necessary grid spacers. Consequently, we chose an idealized ring-cell spacer configuration; ring spacers [Kraemer et al., 1995] have been included in some SCFBR designs [Jevremovic, Oka and Koshizuka, 1996] as well as some boiling water reactor designs. Figure 5-3 provides an overview of the model and the cross section at a grid spacer location. 


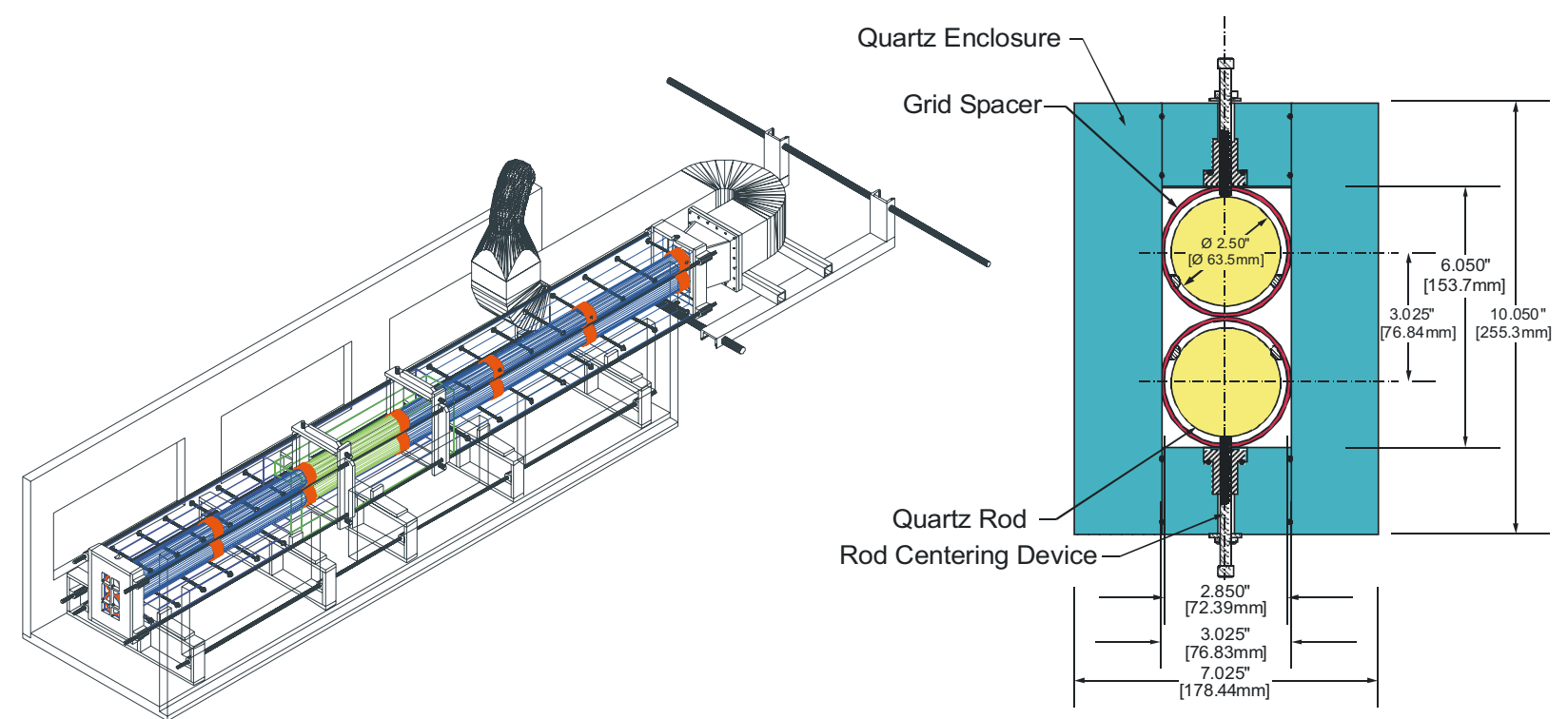

Fig. 5-3. Configuration of model for experiment in the INEEL Matched-Index-of-Refraction flow system, (a) overview and (b) nominal cross section at a grid spacer.

The geometry is scaled to be six to seven times larger than typical fuel pins. Figure 5-4 shows the model assembled before installation in the MIR test section. The rod diameter is 2.50 inches $(63.5 \mathrm{~mm})$ and the axial pitch of the ring-cell spacers is 17.5 inches $(444.5 \mathrm{~mm})$. The pitch-to-diameter ratio $(\mathrm{p} / \mathrm{D})$ is about 1.21 for the simulated fuel rods. The spacers have lengths of 1.75 inches $(44.5 \mathrm{~mm})$ and inside diameters of 2.85 inches $(72.4 \mathrm{~mm})$. Three protuberances, simulating two dimples and a spring, center the rods in the spacer; these protuberances are hemispherical with radii of 0.175 inches $(4.5 \mathrm{~mm})$ as shown in Figure 5-3b. The spacing between the rings was chosen to be thirty times (p-d) to allow significant redevelopment and mixing of the flow; however, in some laminar flows this distance may not be sufficient to become fully-developed before the upstream influence of the next spacer is encountered. The model may be rotated ninety degrees so all three velocity components may be measured with the two-component LDV system. In the measuring region the rods, one grid spacer and side and end walls are fabricated of quartz to match the refractive index of the light mineral oil employed as the fluid.

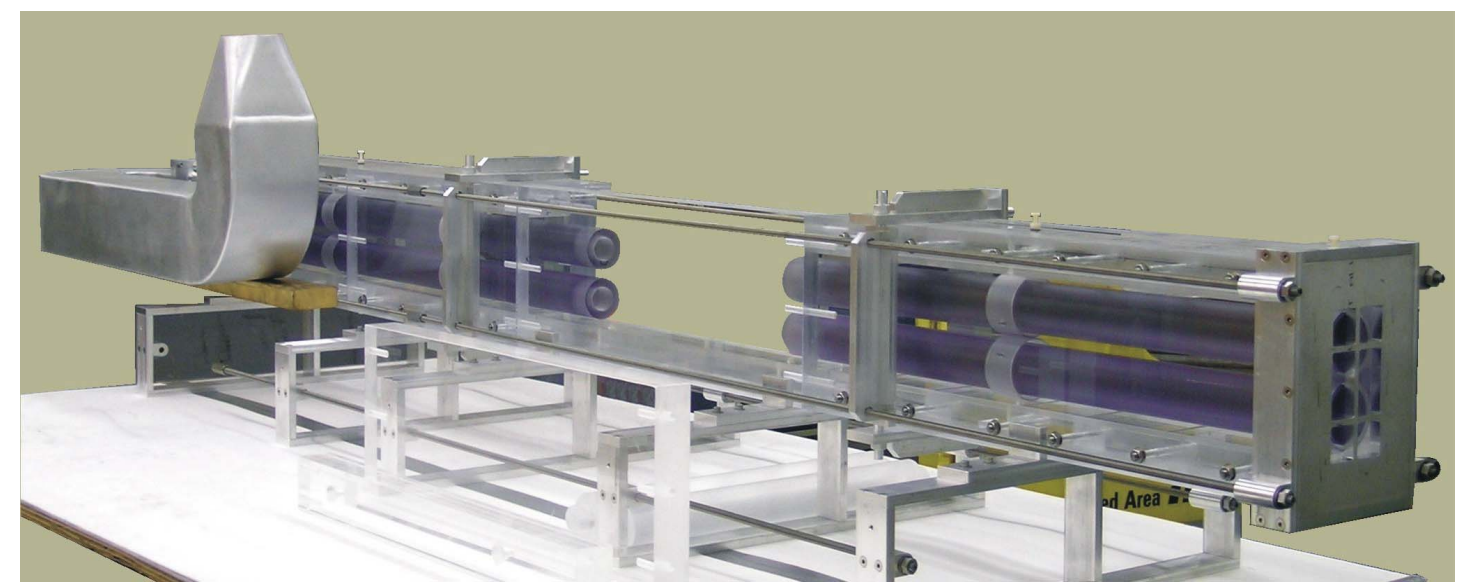

Fig. 5-4. Photograph of model assembled as it appears in MIR test section with refractive indices matched (e.g., the acrylic, polycarbonate, aluminum and steel components are visible).

The model is mounted longitudinally in the MIR test section with flow of the working fluid surrounding it to maintain the desired temperature. Thus, optical access is via the plane side or top walls with the fluid and solid regions inside being held at an equal, constant, uniform value of their refractive indices. Flow enters the model through a chamber containing a honeycomb structure to eliminate swirl; then the abrupt entrance to the flow region 
between the rods assists tripping the flow to turbulent, if not already in that state. Components outside the test section include the pump for the working fluid, the model temperature control system, flow meters and insulation.

Figure 5-5 provides a photograph of the model installed in the MIR test section with the PIV operating (camera and vertical green light sheet) with the oil at the temperature for matching at the $532 \mathrm{~nm}$ wave length. The "unmatched" plastic rods and grid spacers are visible on the left. The quartz rods on the right side are nearly invisible to the "eye" but their locations are outlined by the light sheet. The light sheet is primarily visible where seeding particles scatter light from the fluid above, between and below the rods. The lower grid spacer on the right is quartz while the upper one is plastic but is partly blocked from view by the LaVision PIV camera mounted on the traversing mechanism.
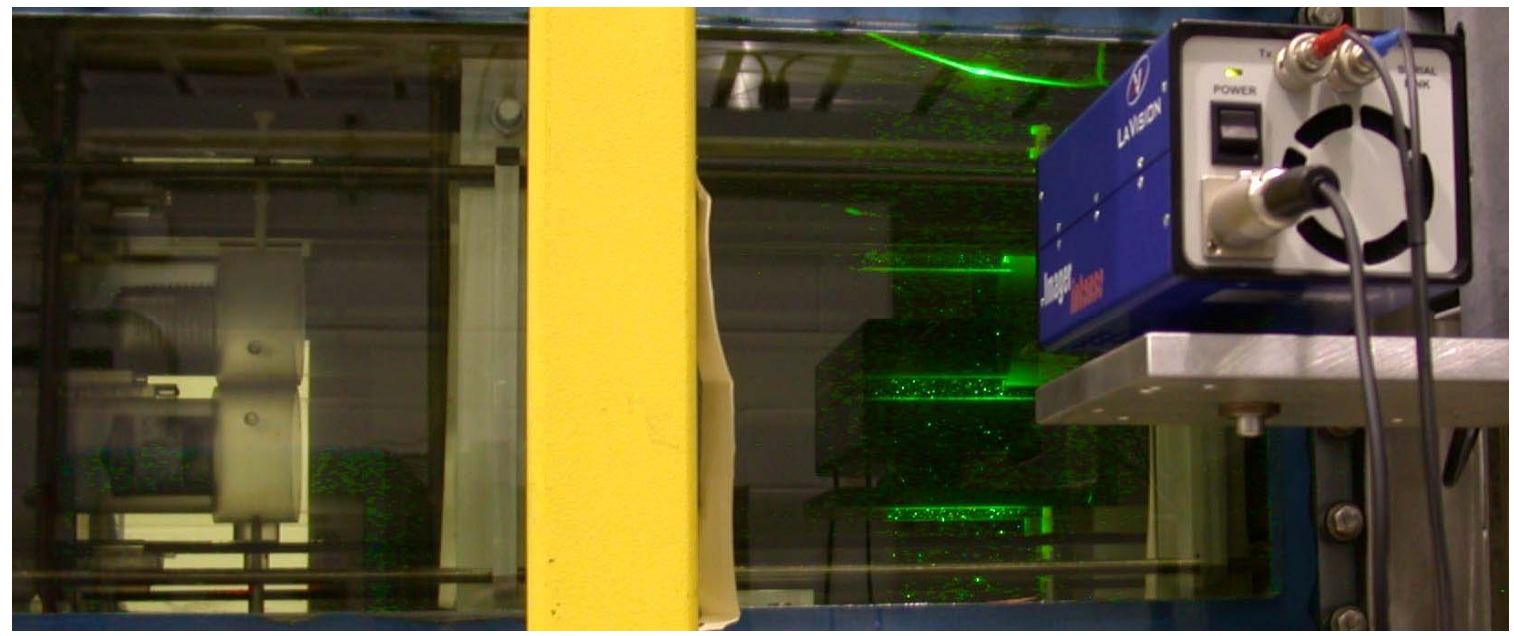

Fig. 5-5. Model installed in MIR test section with PIV system operating.

In situ measurements of model positions were obtained by moving the PIV camera with the precise traversing system (to five $\mu \mathrm{m}$ or better) and adjusting the centered camera crosshairs on the spot to be located [McIroy and Pink, 2005]. Both horizontal and vertical locations could be determined in this manner; the uncertainty in position determination was about $0.1 \mathrm{~mm}$. These measurements plus some on the disassembled model show slight differences from the nominal design, both in cross section and symmetry. From design sketches and tolerances, the streamwise spacing between grid spacers was estimated to be $399.97 \mathrm{~mm} \pm 0.4$ per cent; due to a slight cocking of some spacers, along the vertical centerplane there was a measured variation of about 0.6 per cent in this distance. Diameters of the rods averaged $63.51 \mathrm{~mm}=$ well within design tolerances. The lower rod was measured to be centered within 0.08 per cent of the width and the spacings between the rods and the vertical channel walls agreed with the design within measurement uncertainty. In the vertical plane, the upper rod was slightly low compared to the nominal design (about 0.7 to $1.3 \mathrm{~mm}$ ) and the lower one was slightly higher (1.0 to $0.0 \mathrm{~mm}$ ), yielding a gap between rods of about 7.6 to 7.9 per cent of the channel height rather than the design value of 8.7 per cent (measurement uncertainty is estimated to be about 0.1 per cent in these terms). This gap became slightly larger in the downstream direction. Apparent width (horizontal in Figure 5-3b) was about one per cent smaller than design and channel height was measured to be about 0.7 to 0.4 per cent smaller.

Results The model flow loop was operated to give a flow rate of about $7.30 \times 10^{4} \mathrm{~kg} / \mathrm{hr}\left(1.61 \times 10^{5}\right.$ $\mathrm{lbm} / \mathrm{hr}$ ) and oil temperature of $23.3 \mathrm{C}$ to match the refractive indices at the $532 \mathrm{~nm}$ wave length of the PIV laser. At this temperature the kinematic viscosity is $14.11 \mathrm{cS}\left(1.411 \times 10^{-5} \mathrm{~m}^{2} / \mathrm{sec}\right)$ [Orr, Thomson and Budwig, 1997]. Based on nominal design dimensions, the Reynolds number Re Dh was about 8040 .

Data were acquired in one representative cell between two successive sets of grid spacers where the flow is believed to be streamwise-periodic [Berner, Durst and McEligot, 1984]. Two series of measurements were made. The first, led by Prof. Barton L. Smith of Utah State Univ., employed the PIV system in the two-dimensional mode and covered the entire three-dimensional volume longitudinally between two successive sets of grid spacers. The 
second by Hugh M. McIlroy and Robt. J. Pink of INL concentrated on smaller regions using the three-dimensional mode.

Two-dimensional measurements. The coordinate system employed in this section was centered at the upstream set of grid spacers. The origin of the spanwise direction $(\mathrm{z}=$ horizontal cross stream direction in Figure 5$3 \mathrm{~b}$ and in the installation) was taken to be at the center of the lower rod. The origin of the vertical coordinate (y) was set halfway between the top and bottom walls near the upstream grid spacers; the positive direction is upwards. The streamwise direction $(\mathrm{x})$ was measured from the downstream lower edge of the upper upstream grid spacer. Due to the presence of o-ring seals in the bottom wall (Figure 5-3b), valid measurements are limited to a region about $-35<\mathrm{z}<35 \mathrm{~mm}$ or less.

Measurements of two velocity components ( $\mathrm{u}$ and v) are made using the Particle Image Velocimetry (PIV) system by LaVision, Inc. with a single camera. The light sheet was oriented in a streamwise vertical plane (x-y) and was traversed in the spanwise direction. The camera was mounted so its viewing axis was perpendicular to this plane, i.e., pointing in the spanwise direction. The positive direction for $\mathrm{z}$ was in the direction of the camera view, away from the observer.

The PIV software provides a planar two-component velocity vector field ( $\mathrm{u}$ and $\mathrm{v}$ as functions of $\mathrm{x}$ and $\mathrm{y}$ ) at an instant in time. Each measurement requires a pair of digital images separated by a known time increment. The velocity is calculated by correlating the two images. The flow is seeded with ten $\mu \mathrm{m}$ silver-coated glass spheres. These particles are chosen to be approximately neutrally buoyant, allowing them to follow the flow closely. The particles are illuminated by the laser light sheet during the capture of each image. Cross-correlations are performed on small subregions (interrogation windows) of the image pairs to determine the most likely velocity vector in the plane for that sub region. The interrogation window initially consists of 64 pixels in each direction and these may overlap one another by fifty percent. The resolution and accuracy of the result can be improved by shifting the second window in the estimated direction of the velocity vector by a known amount. A first pass without a shift provides the estimate of how far to displace the second window on the second pass. Multiple passes make it possible to reduce the interrogation window to sixteen pixels, quadrupling the spatial resolution.

With the fifty mm lens, the camera view encompassed the vertical span of the flow region and slightly more than half the distance between successive spacer sets. Therefore, flow through the section was first captured by taking data at an upstream station, moving the camera downstream and taking additional data at the downstream station. Combining the data from the two stations then created a complete picture of the observation section. The data in the region where the upstream and downstream images overlapped were averaged. Data were acquired in this fashion for 33 light sheet planes ( $x-y)$ traversing in the spanwise $(z)$ direction. At each position $(x, y, z), 400$ samples of the instantaneous $u$ and $v$ velocities were collected, from which the flow statistics were calculated. The 33 resulting planes were then combined into one file, which documented the flow through the entire threedimensional measurement region longitudinally between spacers. For each plane, 400 instantaneous flow fields were acquired at a rate of four per second. This rate was based on an estimate of the lowest frequencies likely to be present in the flow and was validated by acquiring sequences of 400 samples at one and two Hz and verifying that the values did not change. A FORTRAN was code used to reduce all data and to tabulate the results into a streamwise series of cross stream (y-z) sets [Smith, 2005].

The bias uncertainty of each instantaneous PIV measurement is estimated as by Adeyinka and Naterer [2005] by noting that PIV measurements are made by determining the displacement of particles over a set time interval, specifically,

$$
u=\frac{\Delta s L_{0}}{\Delta t L_{I}}
$$

where $u$ is a general velocity component, $\Delta t$ is the time interval between the laser pulses, $\Delta s$ is the particle displacement from the correlation algorithm, $\mathrm{L}_{0}$ is the width of the camera view in the object plane in physical coordinates and $\mathrm{L}_{\mathrm{I}}$ is the width of the digital image in pixels (the streamwise direction was chosen). The velocity bias uncertainty based on these values was computed to be about $0.018 \mathrm{~m} / \mathrm{s}$ or 0.3 per cent at $6.1 \mathrm{~m} / \mathrm{s}$. In addition to 
the bias uncertainty of the instantaneous velocity, the fluctuating, turbulent velocity field results in a precision uncertainty in the mean value. For a 95 per cent confidence interval, the precision uncertainty is

$$
P_{u}=1.96 \frac{u_{r m s}}{\sqrt{N}}
$$

where $N$ is the number of independent samples (400), $\mathrm{u}_{\mathrm{rms}}$ is the root-mean-square of the velocity fluctuations and 1.96 is the multiplier for a 95 per cent confidence interval. The precision uncertainty is thus $0.098 \mathrm{urms}_{\mathrm{rms}}$. Typical values of $u_{r m s}$ are $0.3 \mathrm{~m} / \mathrm{s}$ and in some regions, near the spacers, the rms level is as high as $0.5 \mathrm{~m} / \mathrm{s}$. Using the larger value, one finds the precision uncertainty is about $0.0054 \mathrm{~m} / \mathrm{s}$, which is not significant compared to the bias uncertainty.

The mean axial and vertical velocities were calculated with the FORTRAN code mentioned [Smith, 2005]. In the PIV software there are several criteria used to determine the validity of a calculated vector. In the case of an invalid vector, the value of the vector is specified as exactly zero. Also in the software, masks were applied to the portions of the image where the solid model was present, i.e., rods, spacers, walls. The velocity was set identically to zero in these areas. As the instantaneous velocities at each point were summed the number of non-zero values was counted, giving the value of $\mathrm{N}$ (to be used in calculating mean statistics) at that point. If the number of valid vectors at a point was found to be less than half the total number of vectors, the mean velocity at that point was set to zero. With the mean velocities known, the instantaneous velocities were calculated and the Reynolds normal and shear stresses were deduced in turn. With the Reynolds normal stresses known at each point, a two-dimensional "turbulence kinetic energy" was calculated as

$$
T K E=\frac{1}{2}\left(\overline{u^{\prime} u^{\prime}}+\overline{v^{\prime} v^{\prime}}\right)
$$

From the 400 instantaneous $u$ and $v$ velocities collected at each spatial point, the mean streamwise and vertical velocity components were calculated, as well as the in-plane Reynolds stresses (normal and shear) and the turbulent kinetic energy. The final data set is a 1.6 million data point, $155 \mathrm{MB}$ ASCII (TechPlot format) file. The file has columns of $x, y, z, U, V, \overline{u^{\prime} u^{\prime}}, \overline{v^{\prime} v^{\prime}}, \overline{u^{\prime} v^{\prime}}$ and TKE. The streamwise origin $(x)$ is located at the lower downstream edge of the upper upstream grid spacer. The domain of the data set extends slightly upstream of the origin. This file and some images and animations from it are archived on Prof. Smith's internet web site (www.mae.usu.edu/faculty/bsmith/EFDL/KNERI/KNERI.html). For convenience, partial data for three cross stream planes $(\mathrm{x}=32,80$ and $320 \mathrm{~mm})$ are tabulated in his technical report which is also available from this site (http://www.mae.usu.edu/faculty/bsmith/EFDL/KNERI/KNERIreport.doc).

Figures 5-6, 5-7 and 5-8 present an overview of the flow behavior measured in terms of contours of mean streamwise velocity $\mathrm{U}$, mean vertical velocity $\mathrm{V}$ and the two-dimensional "turbulence kinetic energy," respectively. Flow is from left to right and several cross stream planes as well as one near-wall plane are shown in an isometric format. The first cross stream plane is close to the upstream grid spacers (on the web site data and views are available for planes passing through the quartz lower spacer). One sees that the highest streamwise velocities occur there due to the blockage of the spacers reducing the flow area. 


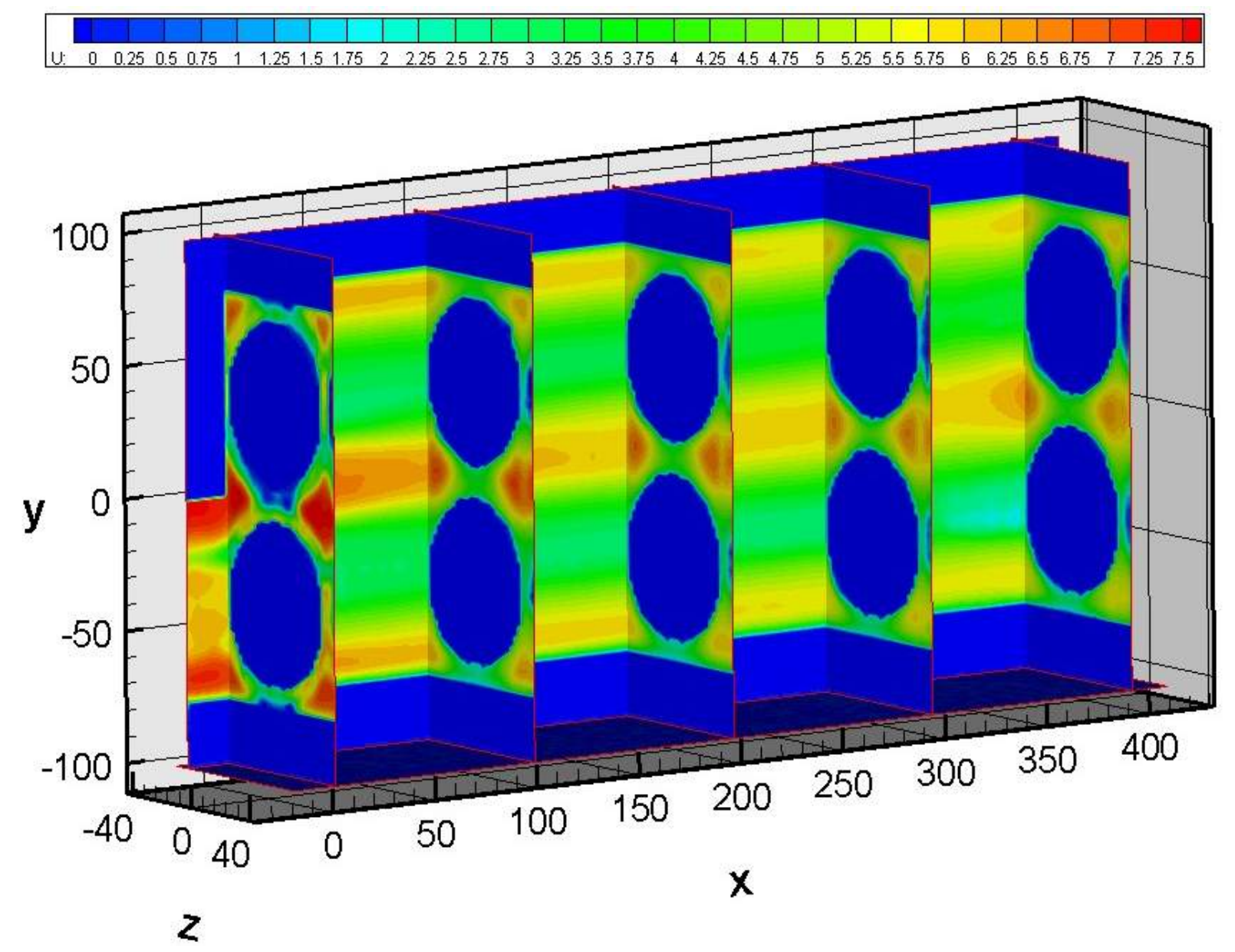

Fig. 5-6. Development of flow between periodic sets of grid spacers in MIR model of a partial SCWR coolant channel, contours of streamwise mean velocity. Velocity in $\mathrm{m} / \mathrm{sec}$ and dimensions in $\mathrm{mm}$.

The centerline of the model $(y=z=0)$ passes through the point where the two spacers meet, so the flow there near $\mathrm{x}=0$ is comparable to that after a backward-facing step. It is retarded and, close to the spacers, one finds recirculation in a small region. The flow redevelops in the streamwise direction and appears to approach a fullydeveloped condition for a rectangular duct enclosing two axial rods. However, in a comparable experiment with thinner grid spacers of a different shape, Rehme [1992] found that his spacer grids affected velocity and turbulence intensity distributions to about forty hydraulic diameters downstream. In the present model, the spacing between grid spacers $\left(\mathrm{L}_{\mathrm{S}} / \mathrm{D}_{\mathrm{h}}\right)$ is less than sixteen so one would not expect full development. In the present experiment, the maximum velocity decreased sharply for about fifty $\mathrm{mm}$ and then more gradually to $\mathrm{x} \approx 350 \mathrm{~mm}$ where it leveled before increasing as the flow approached the downstream grid spacers. For code assessment, the appropriate boundary conditions would be streamwise periodic with the geometry including representation of the spacer ribs (as Prof. Pletcher and his students have done for a ribbed annular geometry in Task 3). An alternate approach would be to use the experimental measurements near the upstream grid spacers as inlet conditions and then to calculate the development downstream to the next grid spacers.

The normalized mean streamwise velocity $\left(U / V_{\text {bulk }}\right)$ shows higher relative velocities everywhere compared to some other studies in similar configurations. Rehme [1992] reported a largest value of $U / V_{\text {bulk }}$ as 1.3 for his four-rod configuration. However, the ratio of flow area through the spacer to the flow area downstream is smaller for the present configuration than for his model. Our greater reduction in flow area would cause the flow to reach higher velocities. 
The vertical mean velocity was generally low as shown in Figure 5- 7. One sees significant vertical velocities immediately beyond the grid spacers, downward from above and upward from below as the flow readjusts into the wakes of the spacers. Downstream these cross stream values are small as the streamwise velocity gradually decreases. Then at the last plane shown, magnitudes increase again as the flow approaches the downstream grid spacers and diverts to pass around them, particularly into the corners of the channel. Comparable behavior is expected of the spanwise component (W) as might be shown by applying the governing continuity equation starting at the wall.
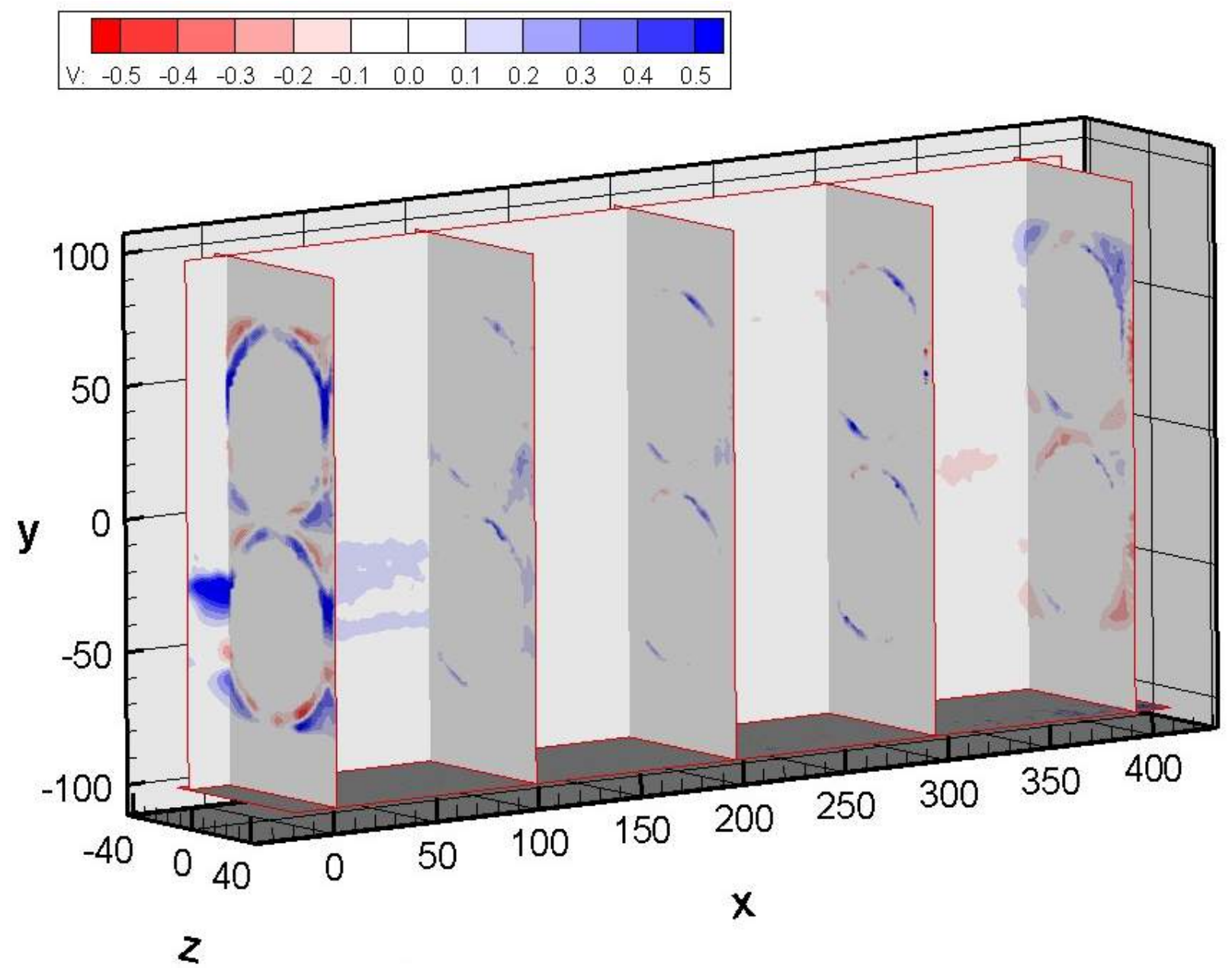

Fig. 5-7. Development of flow between periodic sets of grid spacers in MIR model of a partial SCWR coolant channel, contours of vertical mean velocity. Velocity in $\mathrm{m} / \mathrm{sec}$ and dimensions in $\mathrm{mm}$.

The two-dimensional "turbulence kinetic energy" (say k2) also appears to be low in most of the flow (Figure 5-8). High values occur in the wakes of the grid spacers upstream. If we normalize $\mathrm{k}_{2}$ as a two-dimensional "turbulence intensity" ( $\mathrm{k}_{2}{ }^{1 / 2} / \mathrm{V}_{\text {bulk }}$ ) using the nominal bulk velocity, the highest magnitude is 0.55 , approximately. (These high values bias the color scheme so that variations at lower levels are not clearly discriminated in the figure.) Downstream the higher values appear to be in the near-wall layers around the rods as would also be expected. From Appendix B of the report by Smith [2005], one sees that this intensity varies from about 0.038 to 0.31 at $\mathrm{x}=32 \mathrm{~mm}$. Further downstream (but upstream of the influence from the downstream spacers) it varies from about 0.044 to 0.22 at $\mathrm{x}=320 \mathrm{~mm}$. The lower values are of the same order-of-magnitude as found in the central cores of pipe and duct flows, i.e., away from the surfaces -- as here. 

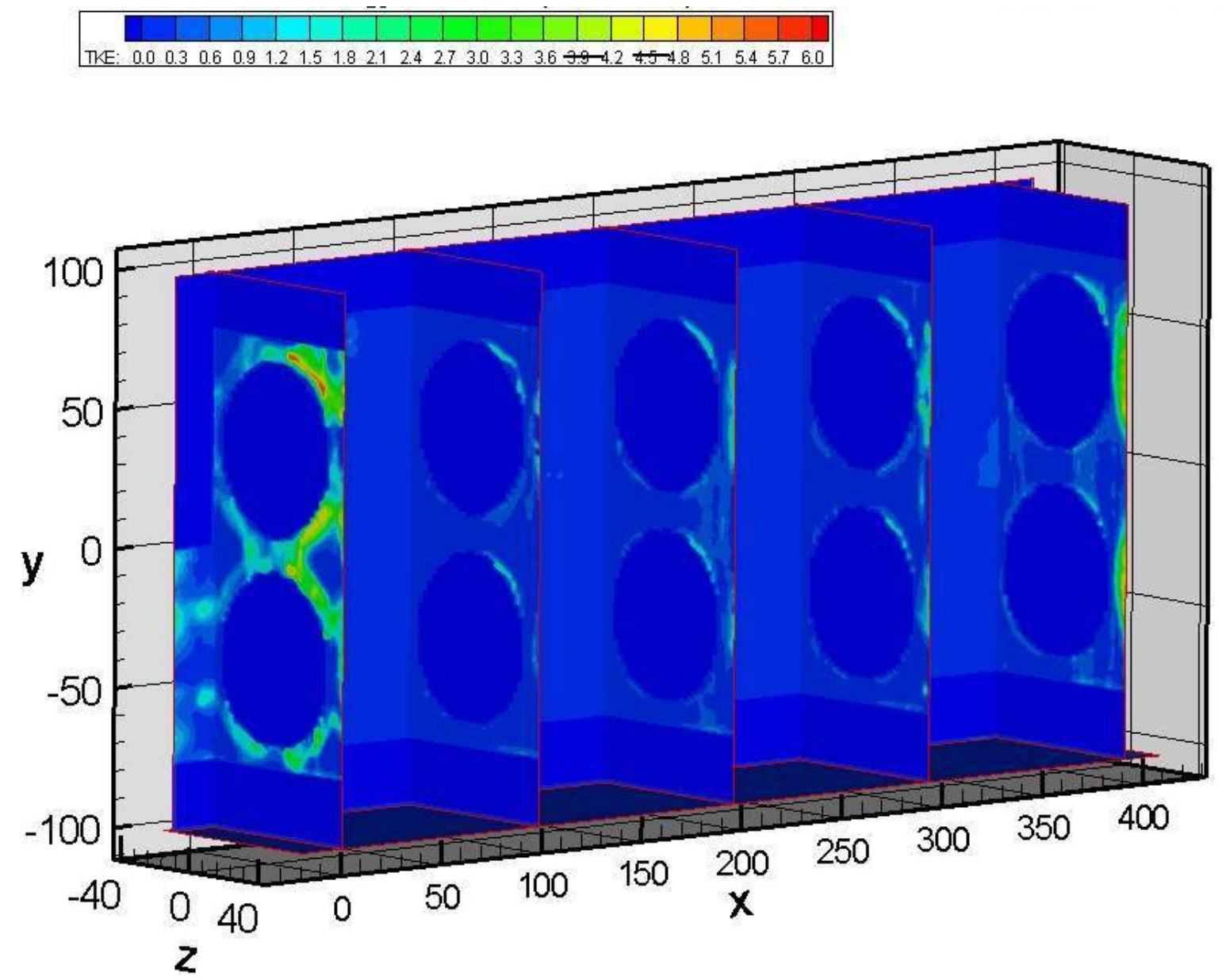

Fig. 5-8. Development of flow between periodic sets of grid spacers in MIR model of a partial SCWR coolant channel, contours of two-dimensional "turbulence kinetic energy." Energy in $\mathrm{m}^{2} / \mathrm{sec}^{2}$ and dimensions in mm.

As noted above, further tabulations of the data are available from the internet web site of Prof. Smith -- for the benefit of CFD code analysts wishing o assess their code performance in a complex geometry which provides features of flow in the coolant channels of some SCWR concepts.

Three-dimensional measurements. Using two cameras in the three-dimensional or stereographic mode, we conducted a second series of measurements at higher resolution but covering a smaller region. Cameras were positioned closer to the model, so the camera view was only about one-third the size of the two-dimensional observations. And the thickness of the light sheet was reduced to about 0.6 to two $\mathrm{mm}$. Therefore the spatial resolution was improved in all three directions.

The three-dimensional PIV mode yields all three instantaneous velocity components over the twodimensional plane of the laser sheet. Consequently, all three mean velocity components, all Reynolds normal and shear stresses and the complete (three-dimensional) turbulence kinetic energy may be calculated with LaVision software or with independent programs as by Prof. Smith and Adam Richards of Utah State Univ.

Since the position measurements indicated model symmetry in the horizontal direction to within measuring uncertainty, data were only acquired for the near half of the flow region. (Examination of profiles of streamwise mean and rms velocities at $z=-27.5$ and $+27.5 \mathrm{~mm}$ confirmed apparent horizontal symmetry.) Measurements were conducted upstream over the range $-18<\mathrm{x}<77 \mathrm{~mm}$ to provide inlet conditions for CFD analysts and downstream at $323<\mathrm{x}<419 \mathrm{~mm}$ where the flow could be considered to be "nearly-developed" before diverting to pass around the downstream spacers. Overlapping streamwise planes of data were acquired at top, center and bottom of the model at 
$\mathrm{z}=0,-5,-10,-15,-17.5,-20,-22.5,-25,-27.5,-30,-32.5$ and $-34 \mathrm{~mm}$. The laser system was operated for about two minutes to obtain 400 data sets for each plane. These raw data (digital photographs) have been archived electronically. The Reynolds number based on design hydraulic diameter was again about 8040 .

LaVision software was employed to deduce mean statistics -- U, V, W, u', v', w', the three Reynolds shear stresses, turbulence kinetic energy $\mathrm{k}$ and others -- for selected observation planes. Figure 5-9 demonstrates the streamwise mean velocity distribution downstream in terms of a series of vertical profiles $U\{y, z\}$. These initial presentations were obtained by "masking" the locations where the light sheet would have been in the transparent rods (therefore zero velocity) according to the design dimensions. Since there were some slight differences between the fabricated dimensions and the design, this masking was not perfect and one can see indications of some anomalous data points and some apparently missing regions.

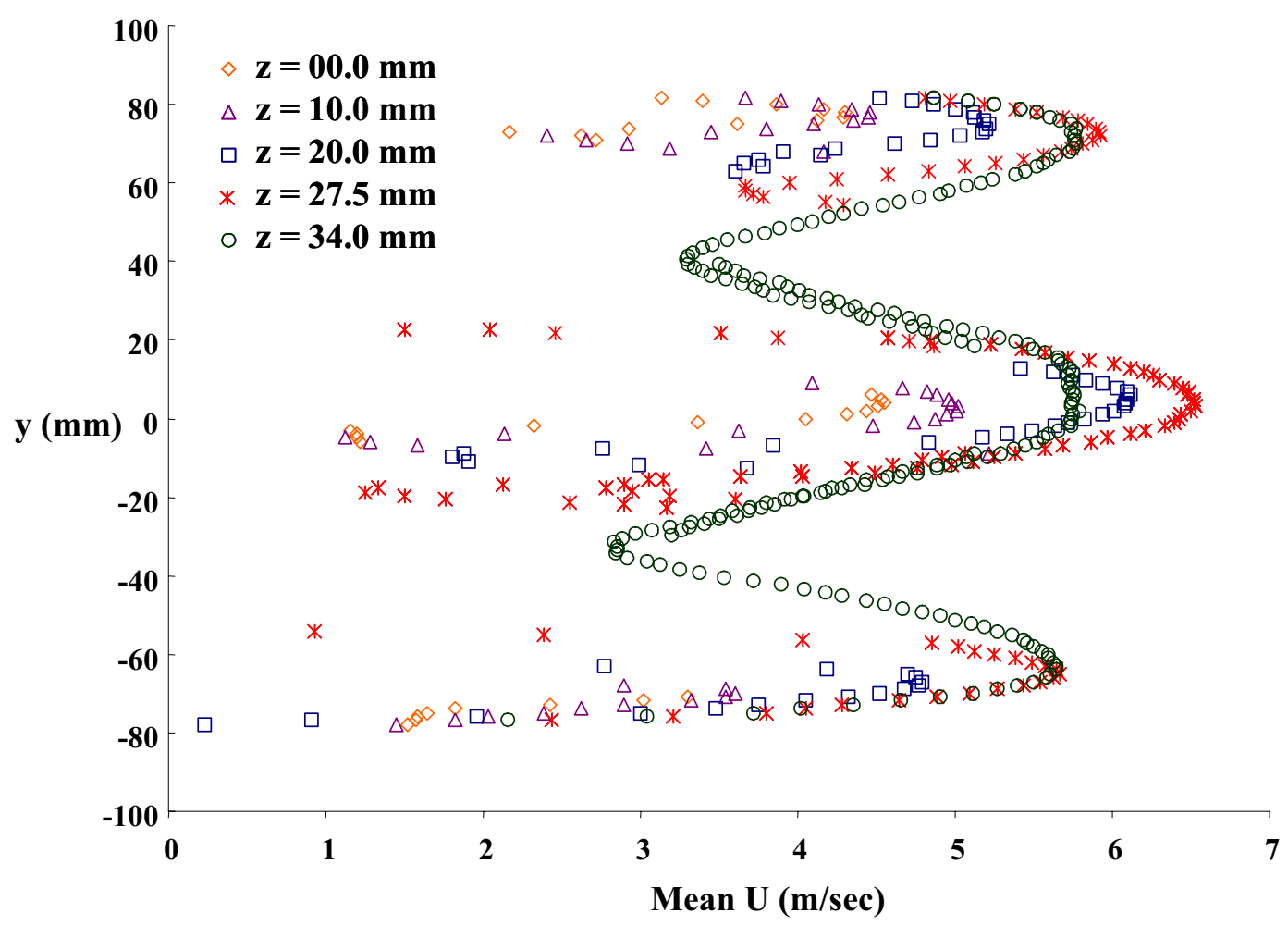

Fig. 5-9. Downstream distribution of streamwise mean velocity $U$ at $x=338 \mathrm{~mm}$. Velocity in $\mathrm{m} / \mathrm{sec}$ and dimensions in $\mathrm{mm}$.

These $\mathrm{U}\{\mathrm{y}\}$ profiles describe flow through the three main flow areas of the model at five vertical planes taken at intervals from an initial plane along the spanwise centerline of the model $(\mathrm{z}=0,-10,-20,-27.5$ and -34 $\mathrm{mm})$. As mentioned, three views were required vertically to cover the height of the overall flow channel. For each plane, the upper view includes approximately the top sixty per cent of the upper rod and the area between the top of the rod and the top of the flow channel, the center view includes the area between the upper and lower rods and about forty per cent of each rod and the lower view includes about sixty per cent of the bottom rod and the area between the bottom of the rod and the bottom of the flow channel. As the laser sheet is traversed from $\mathrm{z}=0$ to $\mathrm{z}=-$ $34 \mathrm{~mm}$, the flow region becomes successively larger until at $\mathrm{z}=-34 \mathrm{~mm}$ the sheet does not intersect the rods (Figure $5-3 b)$.

Maximum mean streamwise velocities increase as $\mathrm{z}$ is varied from the centerplane towards the sidewall until the last plane where it decreases slightly. Most $U\{y\}$ profiles through the center channel (actually subchannel) display comparable profiles except for the mean velocity profile at $\mathrm{z}=-34 \mathrm{~mm}$. Higher maximum velocities observed in the center channel correspond to wider vertical flow widths than in the upper and lower channels. The profiles in the upper flow channel (positive y values) generally have maximum velocities slightly higher than those 
observed in the lower. This result may be a consequence of slight vertical asymmetry in channels of the asassembled model [McIlroy and Pink, 2005] and of the effects of the upstream spacers and channel wall geometry. All profiles shown through the upper and lower flow channels are consistent with this observation.

The mean velocity profile at $\mathrm{z}=-34 \mathrm{~mm}$ displays a maximum velocity about nine per cent lower than that observed for the profile at $\mathrm{z}=-27.5 \mathrm{~mm}$. Additionally, the upper, center and lower channel flows at $\mathrm{z}=-34 \mathrm{~mm}$ are nearly equal with the upper maximum velocity only slightly higher than the center, which in turn is only slightly higher than the lower. The minimum mean velocities observed for the $\mathrm{z}=34$ profile occur at almost exactly the points where the distances between the rods and channel wall are minima (the gap or throat between the edge of the rod and the wall).

Profiles of three-dimensional turbulent kinetic energy k are presented in Figure 5-10. These initial results show the same trends as the two-dimensional results (Figure 5-8). In general, highest values occur near the walls while away from the walls the lowest magnitudes occur, as in the turbulent core of (other) duct flows. However, the magnitudes appear higher than the two-dimensional results even considering the additional velocity component

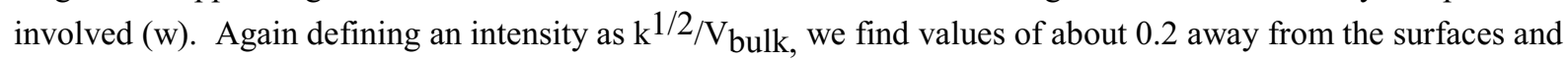
0.6 to 0.9 in the vicinity of the surfaces. For example, the flow at $\mathrm{z}=-34 \mathrm{~mm}$ varies from about 0.16 to 0.27 along a plane that does not approach a rod or side wall. These tke results should be considered to be preliminary; for these data, the data reduction process probably warrants re-examination [Smith, 2005].

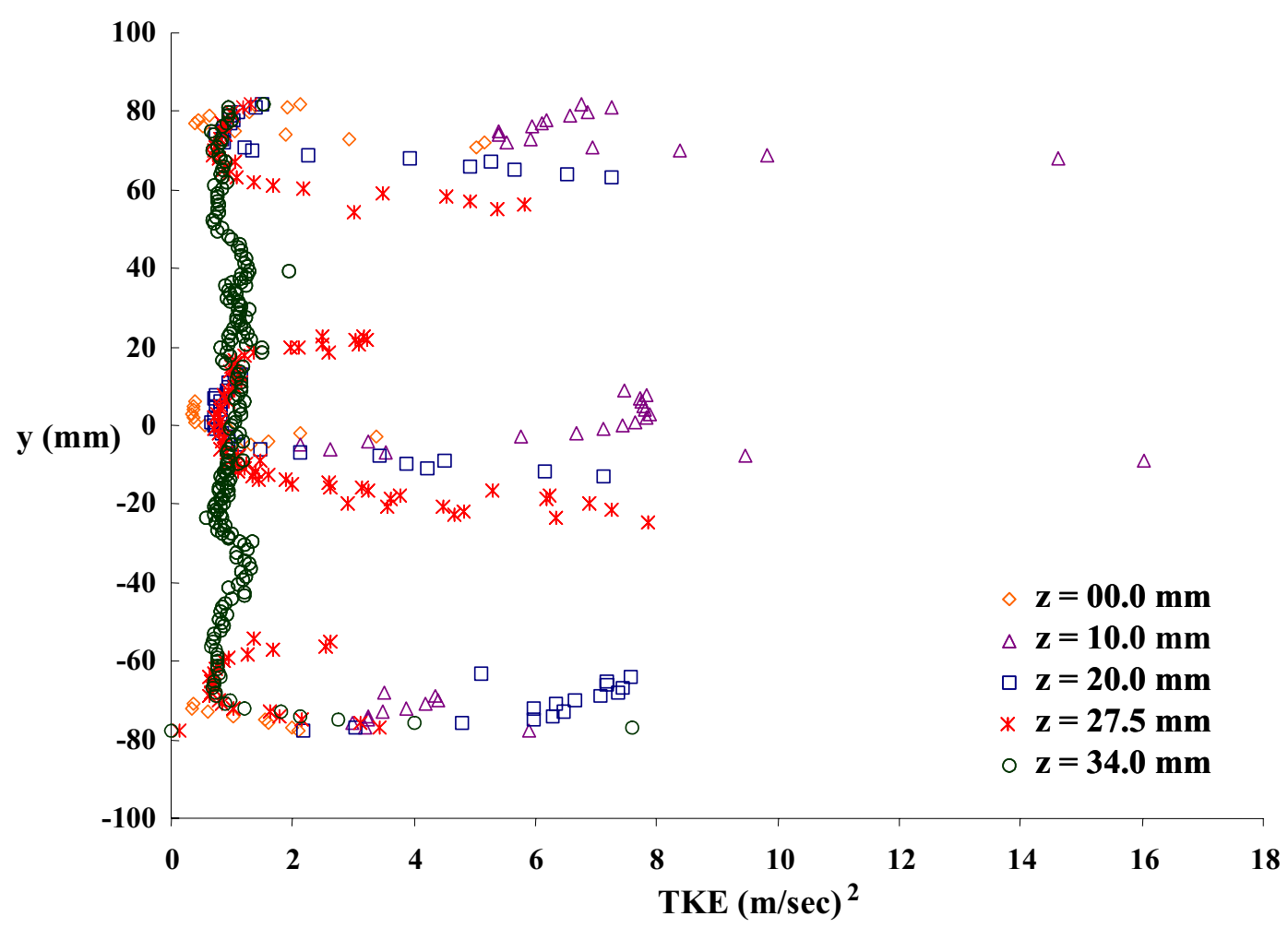

Fig. 5-10. Downstream distribution of turbulence kinetic energy $\mathrm{k}$ at $\mathrm{x}=338 \mathrm{~mm}, \mathrm{k}$ in $\mathrm{m}^{2} / \mathrm{sec}^{2}$ and dimensions in $\mathrm{mm}$.

For CFD code assessment, these three-dimensional data are archived in two forms. Mean statistics from the LaVision software are available for direct comparison in terms of the physical quantities (e.g., $\mathrm{m} / \mathrm{sec}, \mathrm{m}^{2} / \mathrm{sec}^{2}, \mathrm{~mm}$. etc.). For those analysts with capability and desire to deduce mean statistics via their own algorithms, the raw data files are available. Also included in the electronic documentation are tabulations and graphical results used to analyze the data to date. 
Task 6. Miniaturized multi-sensor probes for superheated and supercritical flow, University of Maryland, Prof. J. M. Wallace and Visiting Prof. P. Vukoslavčević (University of Montenegro)

Profs. Wallace and Vukoslavcevic have been developing miniaturized multi-sensor probe $\mathrm{s}$ to measure instantaneous turbulence components in superheated and supercritical flows. These probes are designed to be employed at Seoul National University to measure fluctuating velocity components and temperature in superheated and supercritical flow through a vertical duct (Task 7) for assessment of predictions and to understand the fundamental effects of this heating on the physics of the flow.

The objectives (subtasks) of the University of Maryland group have been to:

1. Design, develop, fabricate and test miniature hot-wire probes to be used in heat transfer experiments to measure simultaneously the streamwise and wall normal velocity components and the temperature in supercritical flow in vertical ducts.

2. Design a calibration and test flow facility that will enable the probes to be calibrated and tested under the same conditions as for the experiments at Seoul National University (Task 7).

3. Create accurate and efficient data reduction algorithms in order to convert digital output from the hot wire anemometer voltages into velocity and temperature components.

4. Create data analysis algorithms that will convert the reduced data from the heat transfer experiments and produce the experimental benchmark information required to assess the DNS, LES and RANS codes that are a major part of this integrated research project.

A five-sensor probe (Two orthogonal X-arrays and one temperature sensor) was first be fabricated and tested in air with an existing calibration facility.

6-1. Probe design Two types of probes have been designed, constructed and tested. As specified in the proposed task, a five sensor probe was designed first and was tested in hot air flow in the existing facility at University of Maryland. This probe could be eventually tested in supercritical flow if the testing facility at the Seoul National University (SNU) is modified for this purpose.

There apparently is no prior work reported in the literature about the response of hot- and cold-wire sensors in supercritical flow, so a simple two-sensor probe, capable of measuring the streamwise velocity component and the temperature, was designed and tested in supercritical flow conditions. With continued progress in the theoretical and experimental analyses of the sensor response and the measurement facility development, eventually probes with as many as five sensors could be tested.

Five-sensor probe. The probes should be designed to measure three velocity components of the turbulent velocity field simultaneously with the turbulent temperature fluctuations with the best spatial resolution possible. The main characteristics of the probe are: the number of sensors and their geometrical arrangements, the uniqueness range, the spatial resolution of the probe and its operational temperature and speed ranges. A five sensor probe, with four velocity sensors in two orthogonal X-arrays and one temperature sensor, has been designed and fabricated. The geometrical configuration is sketched in Figure 6-1. 

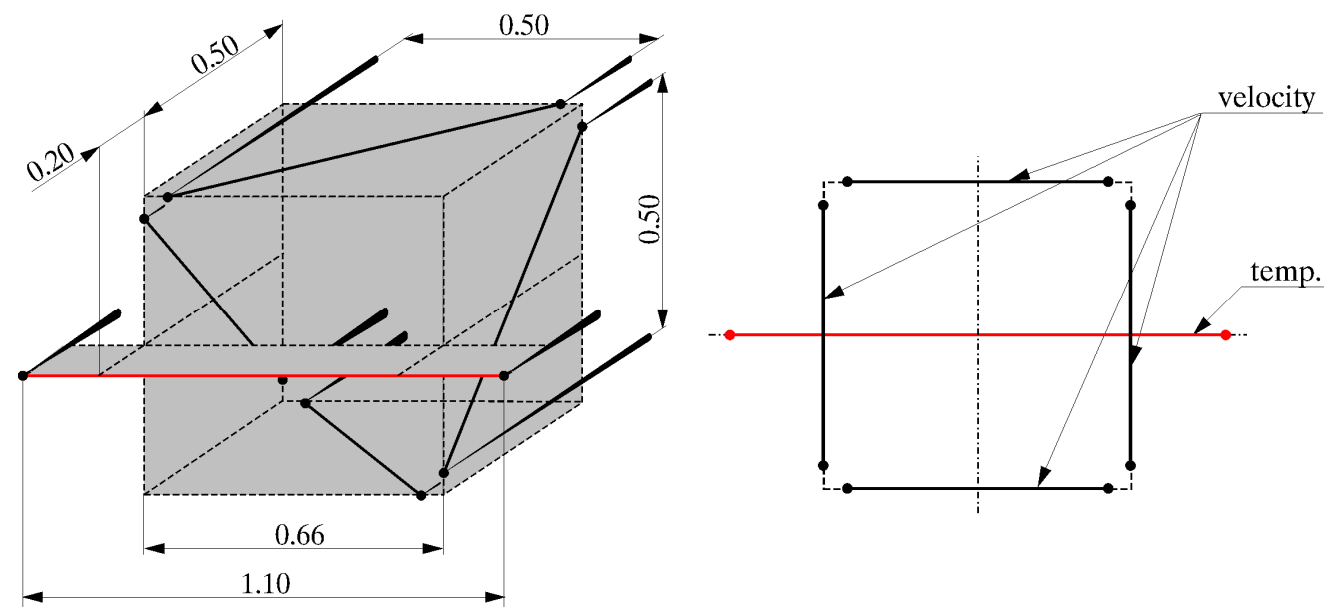

Fig. 6-1. Schematic diagram and front projection of the five-sensor probe.

The probe consists of four heated sensors for the velocity vector measurements and one cold sensor to measure temperature simultaneously. The velocity sensor is operated in the constant temperature (CTA) mode and the cold sensor is operated in the constant current (CCA) mode Three other geometrical configurations that can also measure all three velocity components and temperature are shown in Figure 6-2.

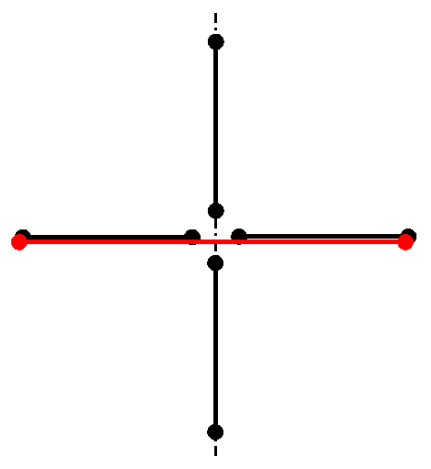

(a)

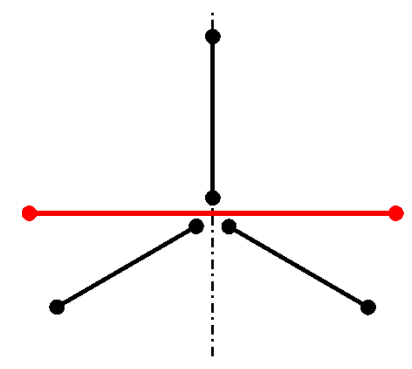

(b)

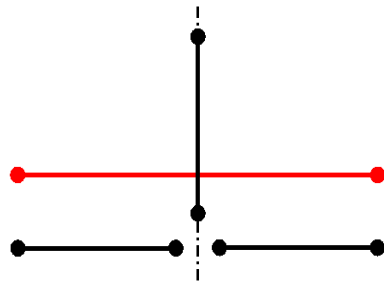

(c)

Fig. 6-2. Different probe geometrical configurations: five-sensor (a), four-sensor $(b, c)$.

The four velocity sensor versions, shown in Figure 6-1 and Figure 6-2a, have, in general, higher uniqueness ranges than the versions with three velocity sensors (Figures 6-2b,c). Although the uniqueness range of the version constructed and tested so far (Figure 6-1) is the same as for the four-sensor probe shown in Figure 6-2a, the thermal influence of the velocity on the temperature sensor should be smaller due to the positioning of the velocity sensors relative to the temperature sensor. It also has better spatial resolution in the vertical direction compared to the version shown in Figure 6-2a. The probe components and sensor materials, as well as the dimensions of the prongs, are the same as for the three-sensor probe described by Vukoslavčević and Wallace [2002].

One of the main problems related to this type of probes is the thermal influence of the velocity sensors on the temperature (cold) sensor. This influence increases with decreasing air speed and the number of velocity sensors as well as increasing their overheat ratio, as described by Vukoslavčević and Wallace [2002]. The difference between the cold sensor's temperature measurement and the air temperature as a function of speed, at overheat ratio of 1.25 , is given in Figure 6-3. There it is seen that the cold sensor is yields a temperature about $1 \mathrm{C}$ above the air temperature at a speed of one $\mathrm{m} / \mathrm{s}$. 


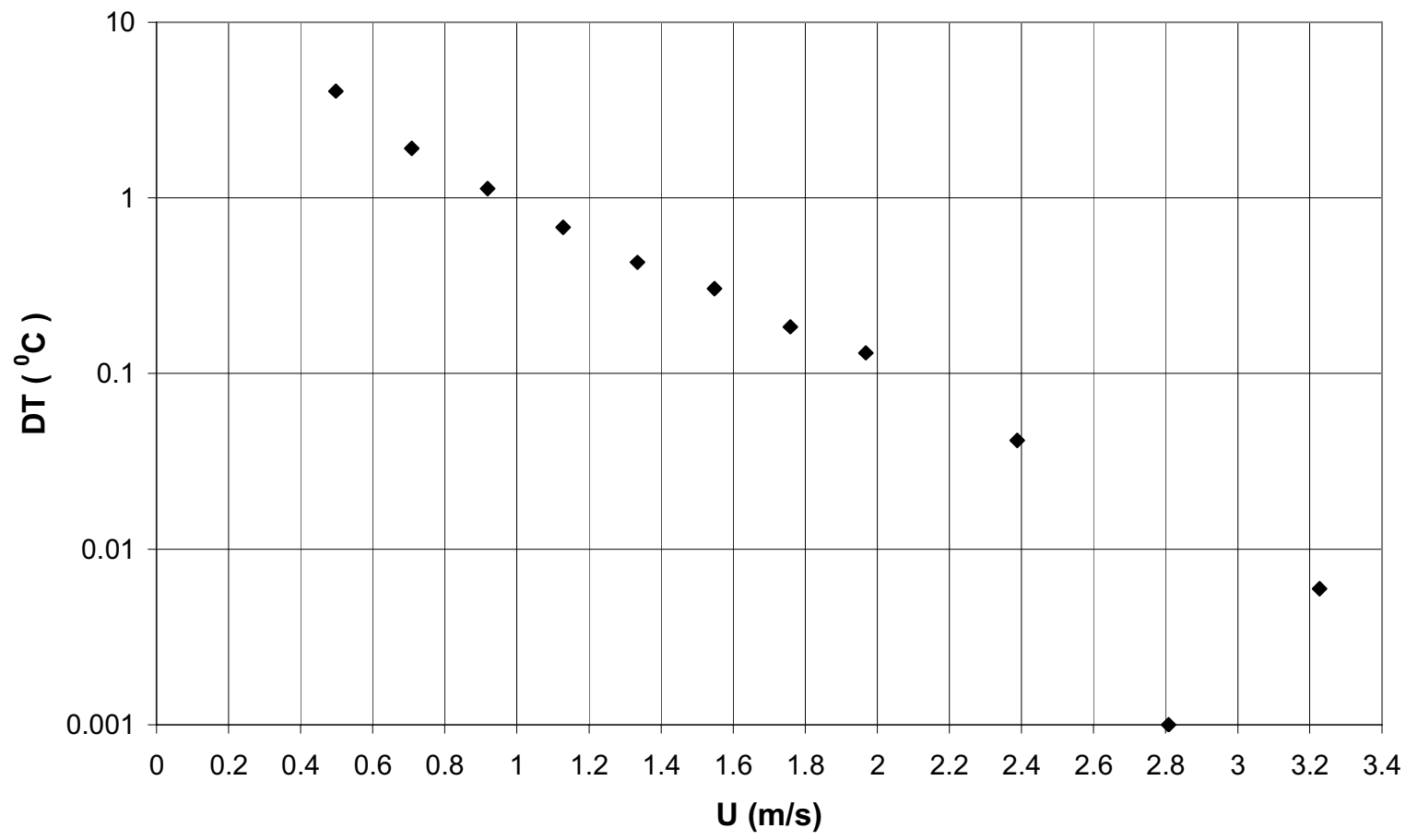

Fig. 6-3. The difference between the cold sensor temperature and the air temperature as a function of air speed.

This speed can be denoted the critical speed (which depends on the desired accuracy of the temperature measurement). It is slightly higher than the critical speed for the three-sensor probe described by Vukoslavčević and Wallace [2002] designed to measure only two velocity components and temperature. Although the number of velocity sensors is doubled for a five sensor probe and, therefore, the number of heat sources is increased, the critical speed does not increase very much compared to that of a three-sensor probe. This result means that the configuration given in Figure 6-1 is the preferred one for full velocity vector measurements in heated flows. It is expected that the critical speed should be much smaller in supercritical carbon dioxide flow, where the probe should be finally used, because the overheat ratio will be much smaller, of the order of 1.1 compared to 1.25 for the air measurements.

Two-sensor probe. The probe consists of two sensors: a hot sensor to measure the streamwise velocity component and a cold sensor to measure the temperature of the flow instantaneously in supercritical carbon dioxide flow. The probe stem and holder were designed to make possible inserting the probe in the high pressure calibration flow loop as well as for moving the probe across the cross section of a pipe with supercritical carbon dioxide flow. The velocity sensor is operated in the constant temperature (CTA) mode and the cold sensor is operated in the constant current (CCA) mode. The sensors are arranged to occupy as small a space as possible in order to achieve the best spatial resolution. They are placed in two planes that cross at an angle of about twenty degrees, as shown in Figure 6-4. 


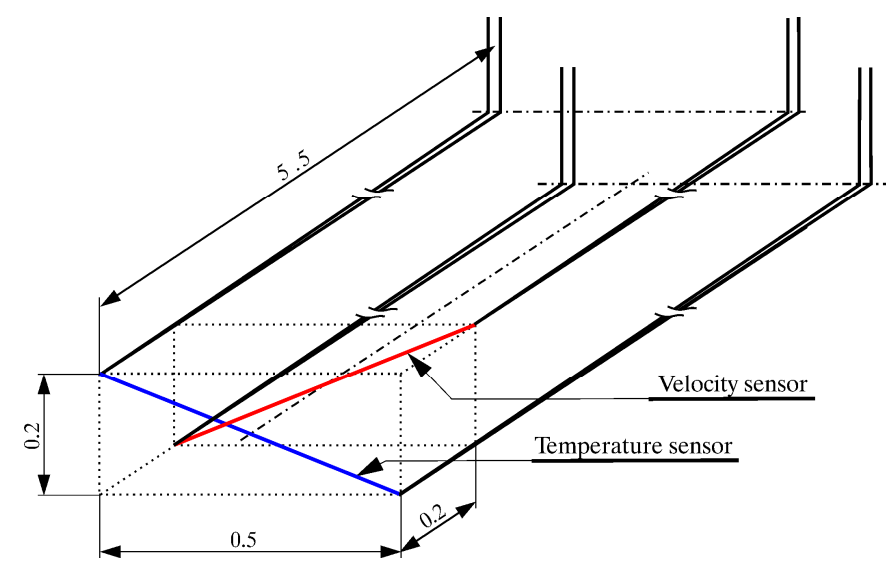

Fig. 6-4. Schematic view of the hot- and cold-wire sensors arrangement.

Platinum $+10 \%$ rhodium wire of $2.5 \mu \mathrm{m}$ diameter is used for both sensors. Tungsten wire of the same diameter also has been tested. To achieve the best frequency response, the smallest possible sensor diameter should be used for the temperature sensor, with the obvious trade-off of increasing the probe's fragility. Alternatively, the frequency response can be compensated if the velocity field is simultaneously measured, as is the case with this probe. The sensor lengths were chosen to be $0.55 \mathrm{~mm}$, giving a length-to-diameter ratio of about 220, which usually is reasonable to obtain a good cooling law for the velocity sensor. The supporting prongs are made of stainless steel. They are $0.25 \mathrm{~mm}$ at the base and taper to about $60 \mu \mathrm{m}$ at their tips. These dimensions were chosen to avoid prong vibration as much as possible in the relatively high-density supercritical fluid, to reduce the blockage of the flow and also to reduce the thermal boundary layer around the prongs of the temperature sensor. The choices were based on our previous experience in constructing probes to measure two velocity components and temperature simultaneously in hot air flows, as described in Vukoslavčević and Wallace [2002]. The prongs were bent nearly at right angles in order to position the sensors as close as possible to the wall. They were placed in a ceramic tube of $1.2 \mathrm{~mm}$, which was then sealed in a stainless steel tube. Different types of epoxy sealants have been tested to be resistant to high pressure and to ensure the sealing between the prongs, ceramic tube and probe body. A special P-2 epoxy, used for strain-gage sensors, turned out to be the most convenient. The temperature sensor was placed only $0.2 \mathrm{~mm}$ upstream of velocity sensor. This small spacing is possible due to the relatively small overheat ratio of the velocity sensor used for these measurements. Otherwise, the thermal energy from the hot velocity sensor could influence the cold temperature sensor. A special gland, shown in Figure 6-5, was mounted on the probe during its construction. By appropriate tightening of the gland, a proper sealing between it and the pipe, as well as between the gland and the probe, could be made. In order to test different calibration parameters under the same flow conditions, another probe with two nearly identical parallel velocity sensors also has been designed and constructed.

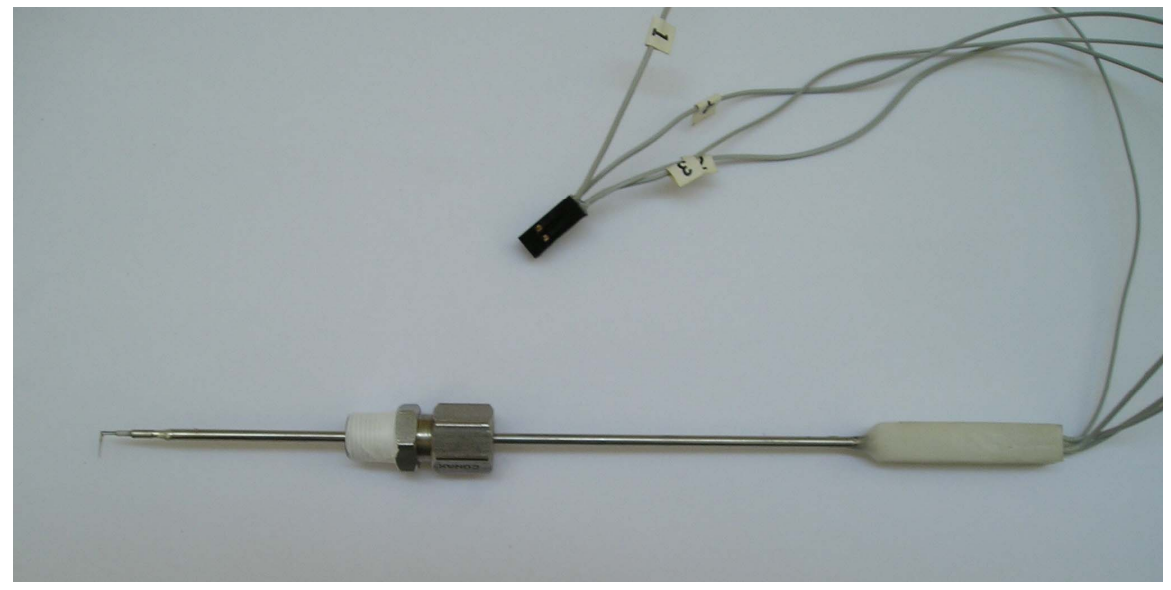

Fig. 6-5. Photo of the probe assembly. 
6-2. Calibration and test facility The fabrication of the calibration and testing facility was planed to be performed at Seoul National University, Korea. In order to accelerate testing of the probe and development of the data reduction algorithms, we decided to construct and fabricate it at the University of Maryland, USA.

The calibration loop should be capable of providing any state condition $(\mathrm{p}, \mathrm{T})$ in the supercritical region, by varying the temperature under constant pressure, by varying the flow rate under constant pressure and temperature and by generating one and two-dimensional flow fields. It should also provide the possibility for accurate measurements of the flow speed, pressure and temperature. The dimensions of the loop should be as small as possible for safety and pollution considerations as well as to minimize thermal inertia and to provide for fast regulation. A design that meets these requirements is shown in Figure 6-6.

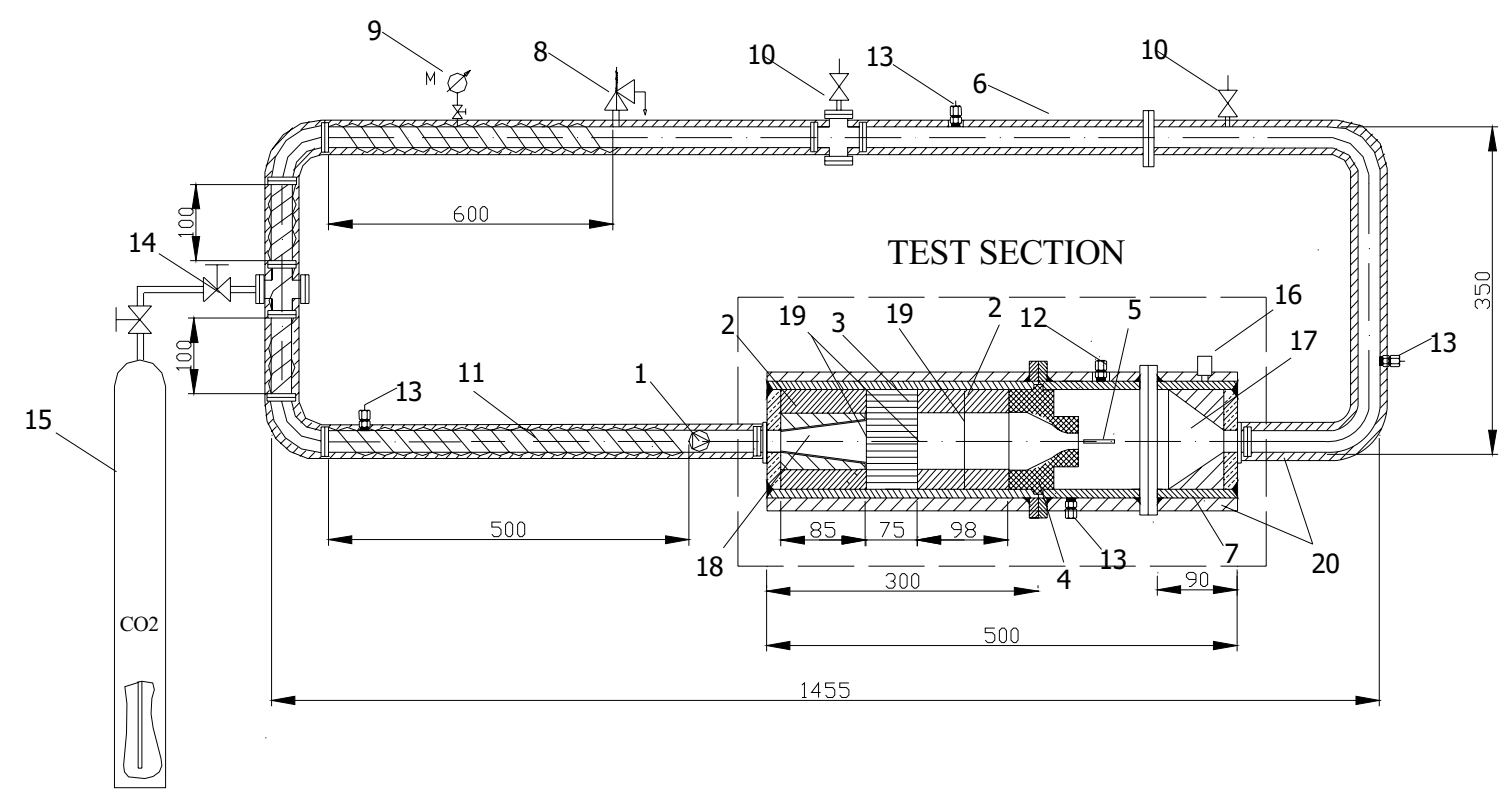

Fig. 6-6. Calibration and testing flow loop: 1 - pump; 2 - aluminum shell; 3 - honeycomb; 4 - nozzle; 5 - probe; 6 pipe DN20 ( $\varnothing 25 \times 3.2 \mathrm{~mm}) ; 7$ - pipe DN80 ( $\varnothing 94 \times 7.1 \mathrm{~mm}) ; 8$ - relief valve; 9 - manometer; 10 - valve; 11 - electric heater; 12 - gland; 13 - transducer gland for thermocouples; 14 - valve; $15-\mathrm{CO}_{2}$ bottle; 16 - pressure transducer; 17 - contraction; 18 - diffuser; 19 -screens; 20 - insulation.

The calibration loop consists of a test section with a cubic nozzle profile at the downstream end to ensure a uniform, low turbulence flow at the nozzle exit, a return flow loop to provide for flow recirculation, a heater to warm and to pressurize the carbon dioxide and a gas bottle to supply carbon dioxide. The probe is mounted in a gland in front of the nozzle, as seen in a close-up drawing of the test section in Figure 6-7. 


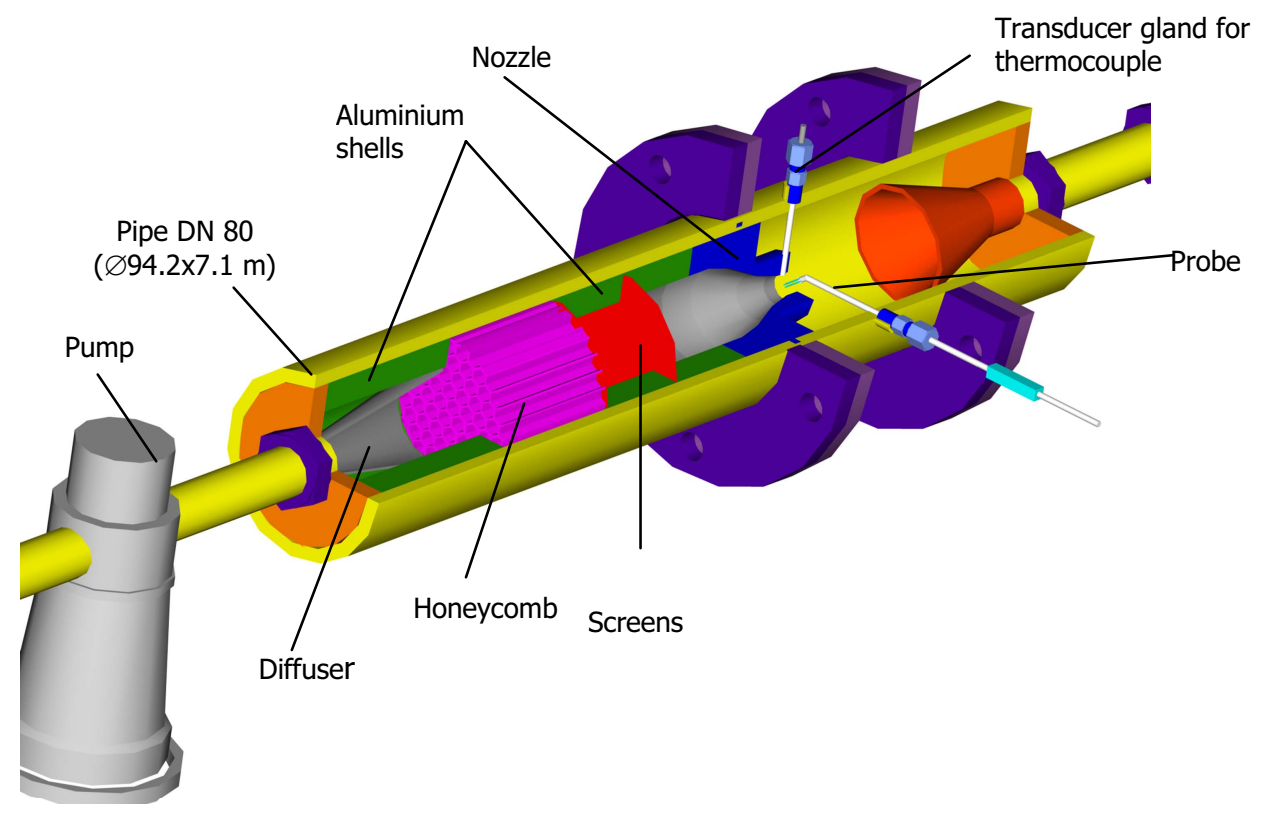

Fig. 6-7. Schematic view of the test section.

The loop must be completely sealed so that the constant pressure can be maintained while the speed and temperature are varied. The sealing of the flange connections is made with O-rings placed in groves with extremely smooth surfaces in the flanges. Sensor leads are inserted through the wall using special glands that sustain pressures of over 100 bars. A magnetic drive gear pump, without any mechanical connection between the pump and motor, was chosen to provide the sealing and a variable, pulseless flow rate. A photograph of the assembly is shown in Figure 6-8, and a photograph of the probe mounted in front of the nozzle in Figure 6-9.

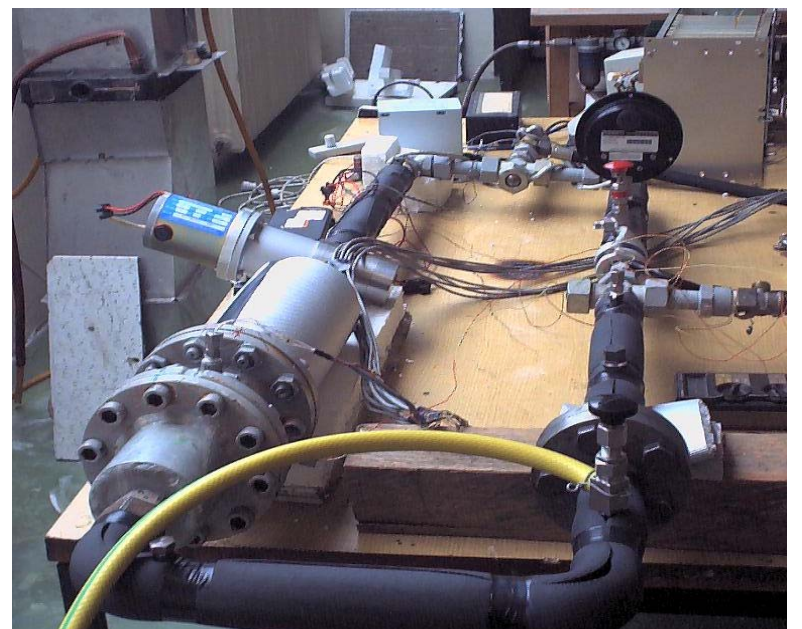

Fig. 6-8. Photograph of the installation.

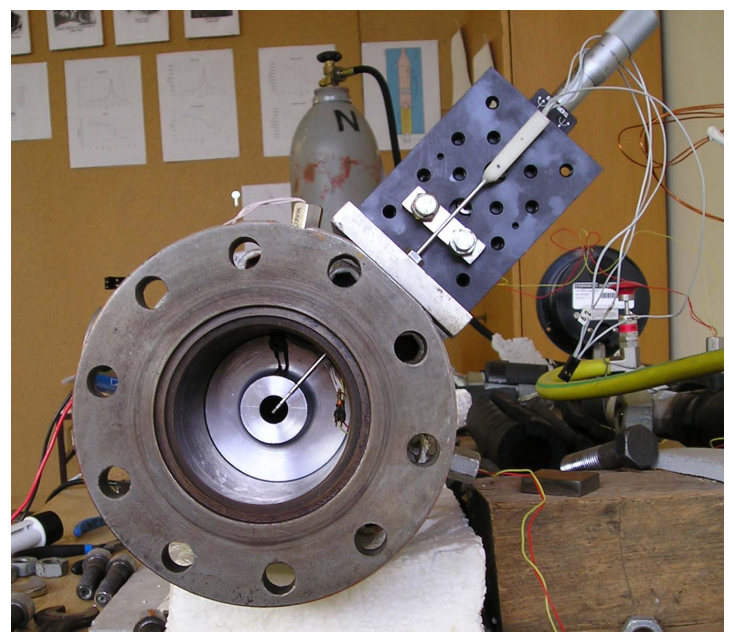

Fig. 6-9. The probe mounted in front of calibration nozzle and connected to traverse mechanism.

The flowrate is varied over the desired ranges by selecting the rotation rate of the variable speed motor. The flow rate is a linear function of the pump rotation rate and the number and dimensions of the gears. Due to the small pressure difference between the pump inlet and outlet, backflow through the pump is negligible, negating the need for an expensive flow meter. The $\mathrm{CO}_{2}$ flow speed at the nozzle exit, as a function of the motor rpm, is shown in Figure 6-10. 


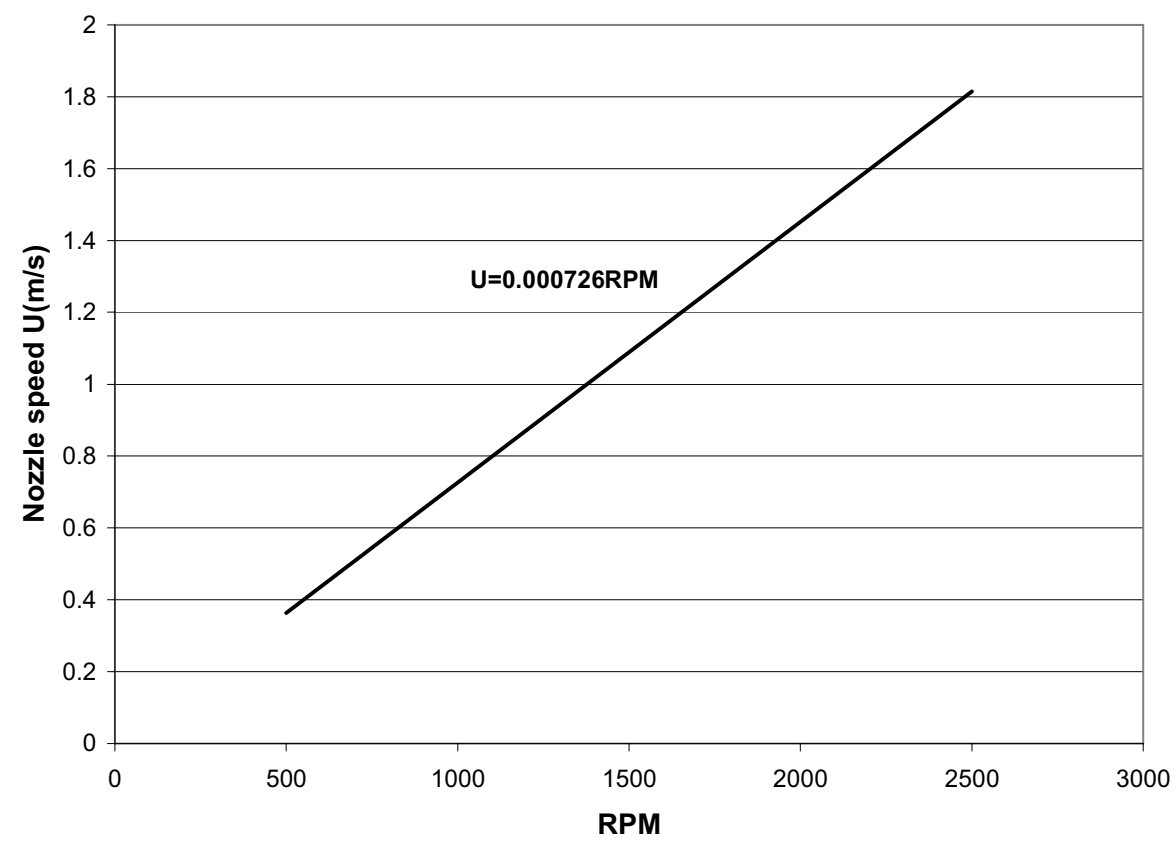

Fig. 6-10. $\mathrm{CO}_{2}$ flow speed at the nozzle exit as a function of motor rpm.

Any desired state condition ( $\mathrm{p}, \mathrm{v}, \mathrm{T}$ ) can be reached by first filling the installation of known volume $\mathrm{V}$ by an amount $\mathrm{M}$ of $\mathrm{CO}_{2}$ to obtain the desired specific volume. The pressure in the installation is then equal to the pressure in the bottle and this pressure depends on the room temperature. The specific volume depends on the amount of $\mathrm{CO}_{2}$ filled in the flow loop. By heating the sealed loop, any temperature or pressure can be reached with constant specific volume, as illustrated by line 1 - 2 in Figure 6-11.

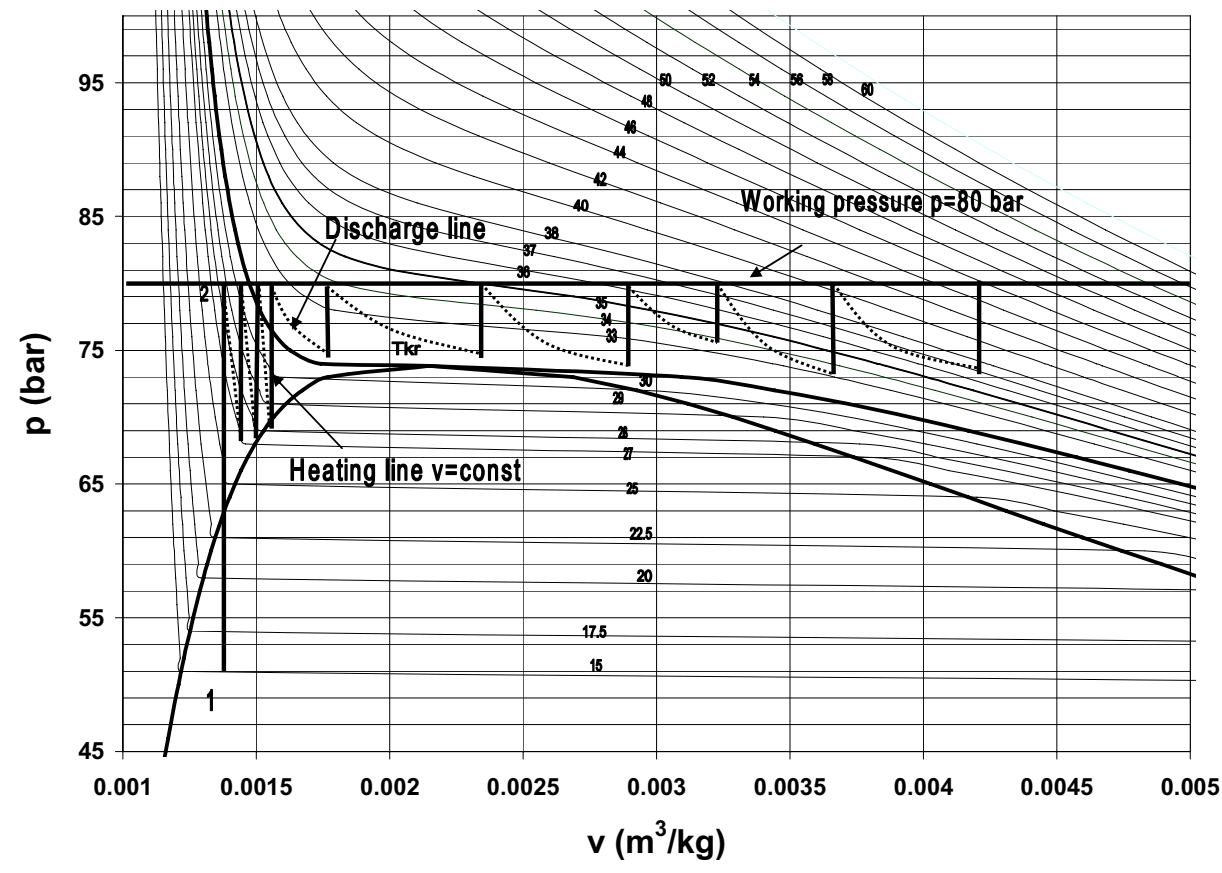

Fig. 6-11. Process of temperature variation under constant pressure in $\mathrm{p}, \mathrm{v}$ diagram. 
To vary the temperature with constant pressure, it is necessary to release an amount of $\mathrm{CO}_{2}$ from the flow loop. This action can be done by opening a valve and directing $\mathrm{CO}_{2}$ into the bottle or out into the atmosphere. The shapes of the discharge lines, shown in Figure 6-11, depend on the manner the $\mathrm{CO}_{2}$ is released from the bottle: e.g., with a fully- or a partially-open valve and with the heater on or off. Heating the installation again after a given mass of $\mathrm{CO}_{2}$ has been released will return the fluid to the desired pressure. The temperature change will depend on the amount of $\mathrm{CO}_{2}$ released and the temperature variation can easily be achieved in steps of $0.5-10{ }^{\circ} \mathrm{C}$. For example, reducing the pressure by 10 bars, at a temperature of $35^{\circ} \mathrm{C}$, and heating the $\mathrm{CO}_{2}$ back to 80 bars will increase the temperature by about $1{ }^{\circ} \mathrm{C}$. By holding the temperature constant at each step along the line of constant pressure and varying the flow rate, the probe response to variable speed with constant pressure and temperature can be determined.

The five-sensor probe has been tested in the existing facility developed in the previous NERI project "Fundamental thermal fluid physics of high temperature flows in advanced reactor systems."'[McEligot et al., 2002] A special device to pitch and yaw the probe, shown in Figure 6-12, has been added to the existing facility.
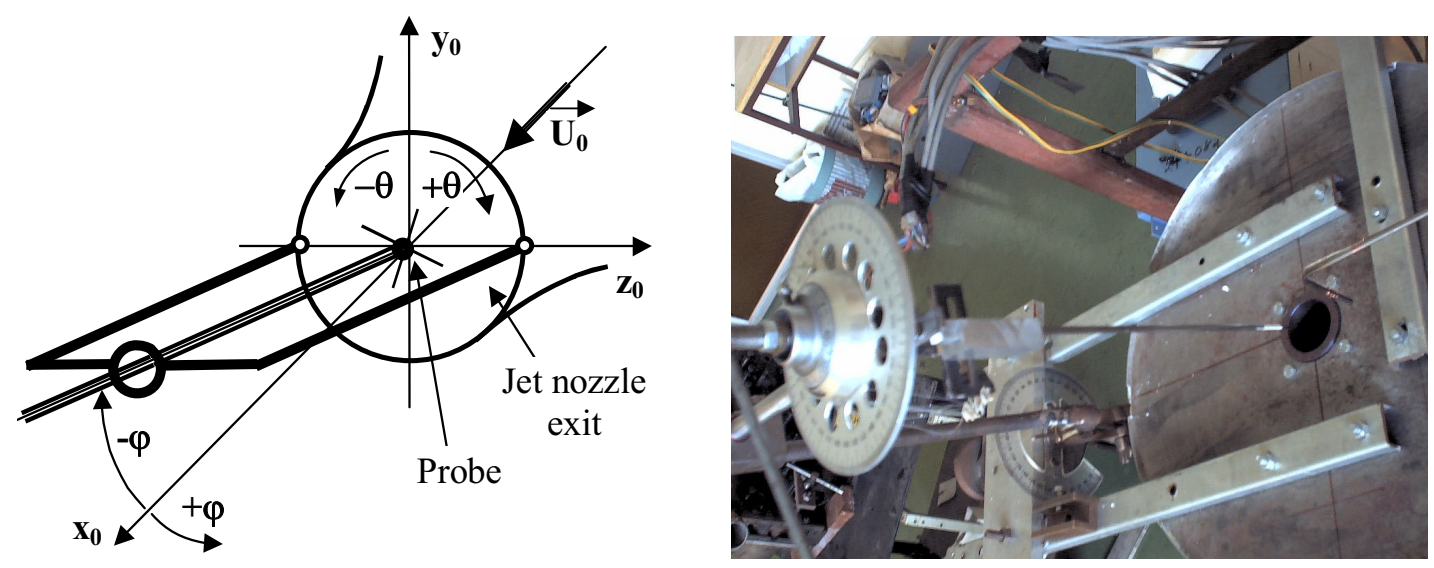

Fig. 6-12. The schematic view and photo of the pitch-yaw apparatus.

\section{6-3. Data Reduction Algorithm - Testing the Accuracy of the Probes}

Five-sensor probe in hot air flow. Based on Jorgensen's [1971] definition of an effective cooling velocity and relating it to a fourth-order polynomial expansion of the anemometer output voltage, the following expression

$$
\begin{aligned}
& B_{i 0}+B_{i 1} E_{i}+B_{i 2} E_{i}^{2}+B_{i 3} E_{i}^{3}+B_{i 4} E_{i}^{4}= \\
& U_{i}^{2}+A_{i 1} V_{i}^{2}+A_{i 2} W_{i}^{2}+A_{i 3} U_{i} V_{i}+A_{i 4} U_{i} W_{i}+A_{i 5} V_{i} W_{i}
\end{aligned}
$$

is obtained for each of the four sensors $(i=1,2,3,4)$. The parameters $A_{i j}$ and $B_{i j}$ will be functions of temperature in a varying temperature flow. By placing the probe in a nominally irrotational calibration jet flow at a given temperature and pitching and yawing it, and using the apparatus shown in Figure 6-12, n different flow realizations $\left(\mathrm{U}_{\mathrm{in}}=\mathrm{U}_{\mathrm{on}}, \mathrm{V}_{\mathrm{in}}=\mathrm{V}_{0 \mathrm{n}}\right.$ and $\left.\mathrm{W}_{\mathrm{in}}=\mathrm{W}_{0 \mathrm{n}}\right)$ were induced in order to determine the forty calibration constants (ten for each sensor) at a given temperature. The number $n$ should be, of course, equal or greater then ten.

The probe was first tested at room temperature. The agreement between the simultaneously induced (imposed) and measured of $\mathrm{U}, \mathrm{V}$ and $\mathrm{W}$ components is excellent. The $\mathrm{U}$ and $\mathrm{V}$ component comparisons are shown in Figure 6-13 and Figure 6-14. 


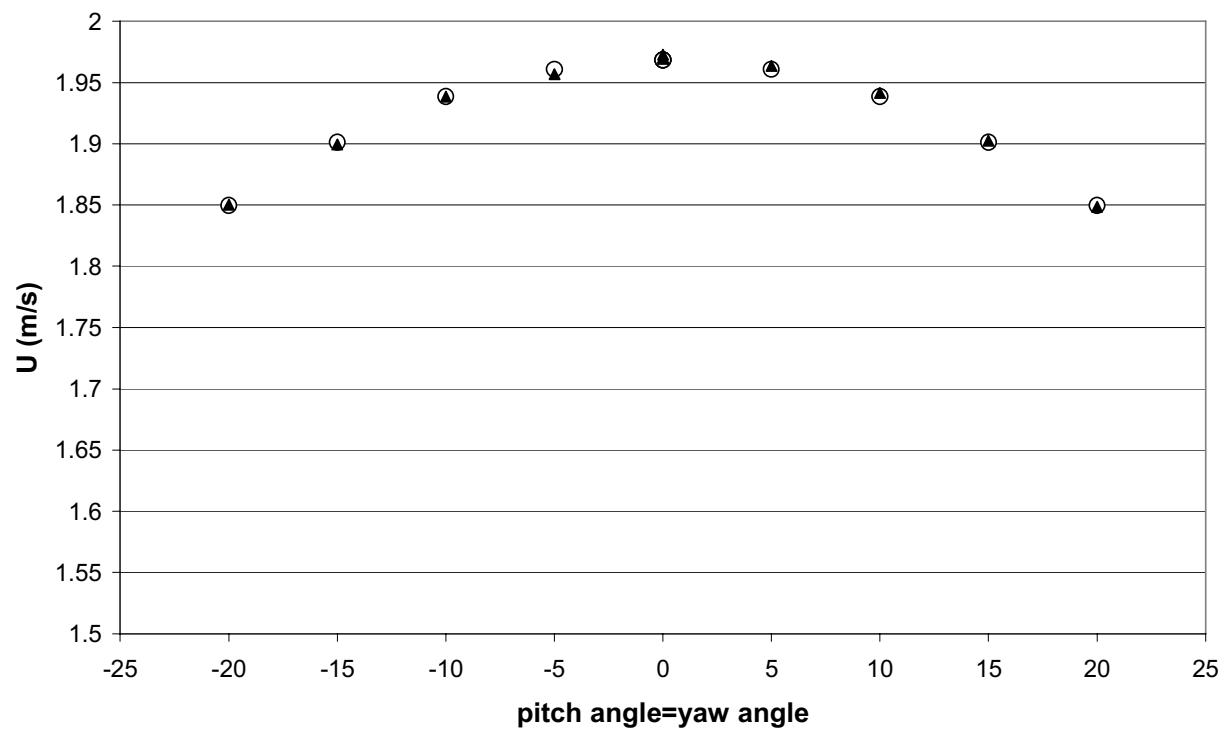

Fig. 6-13. The induced (O) and measured (A) U velocity component.

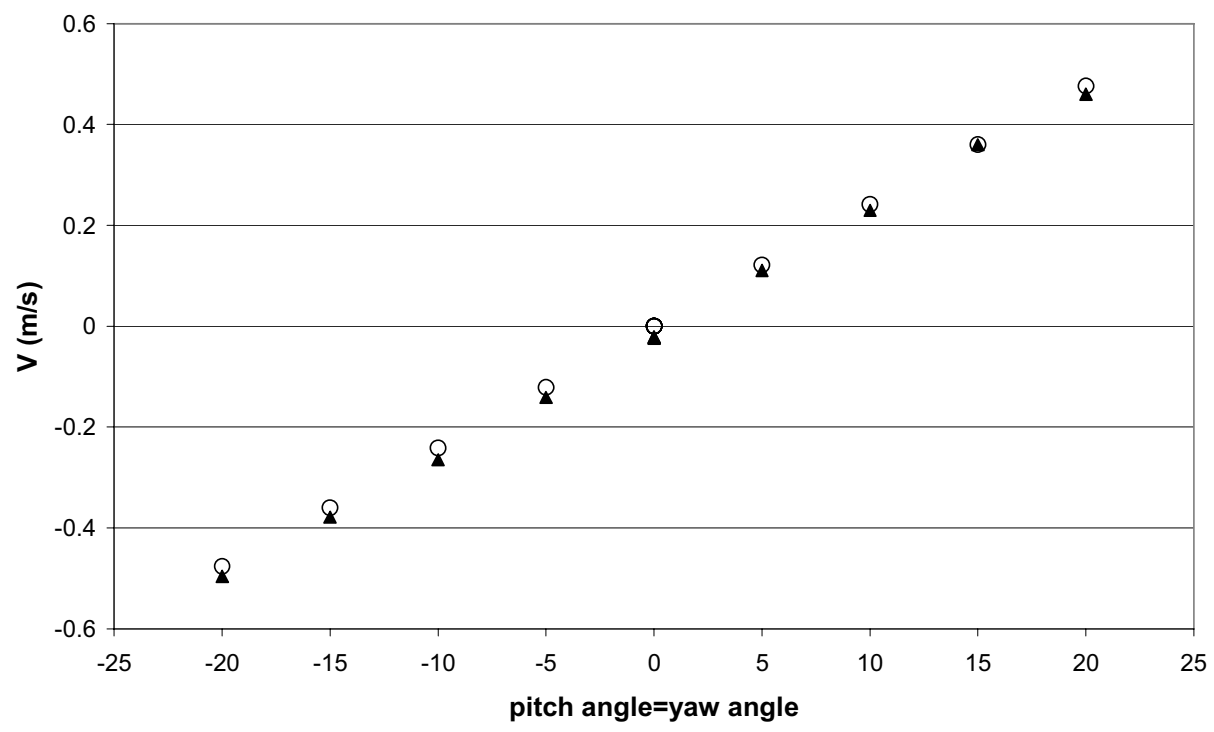

Fig. 6-14. The induced $(\mathrm{O})$ and measured $(\mathbf{A})$ values of $\mathrm{V}$ velocity component.

The results obtained for the $\mathrm{W}$ component are similar to those of the $\mathrm{V}$ component. The agreement between the induced and measured values is within an absolute error of two $\mathrm{cm} / \mathrm{s}$ (approximately one percent of $\mathrm{U}$ ).

The response of the velocity sensors was also tested in the air at temperatures up to $80 \mathrm{C}$. This value is above the highest temperature expected in the supercritical $\mathrm{CO}_{2}$ loop to be used at the Seoul National University. Shown in Figure 6-15 are values of the measured velocity components at $80 \mathrm{C}$ compared to values induced by the calibration and test jet. 


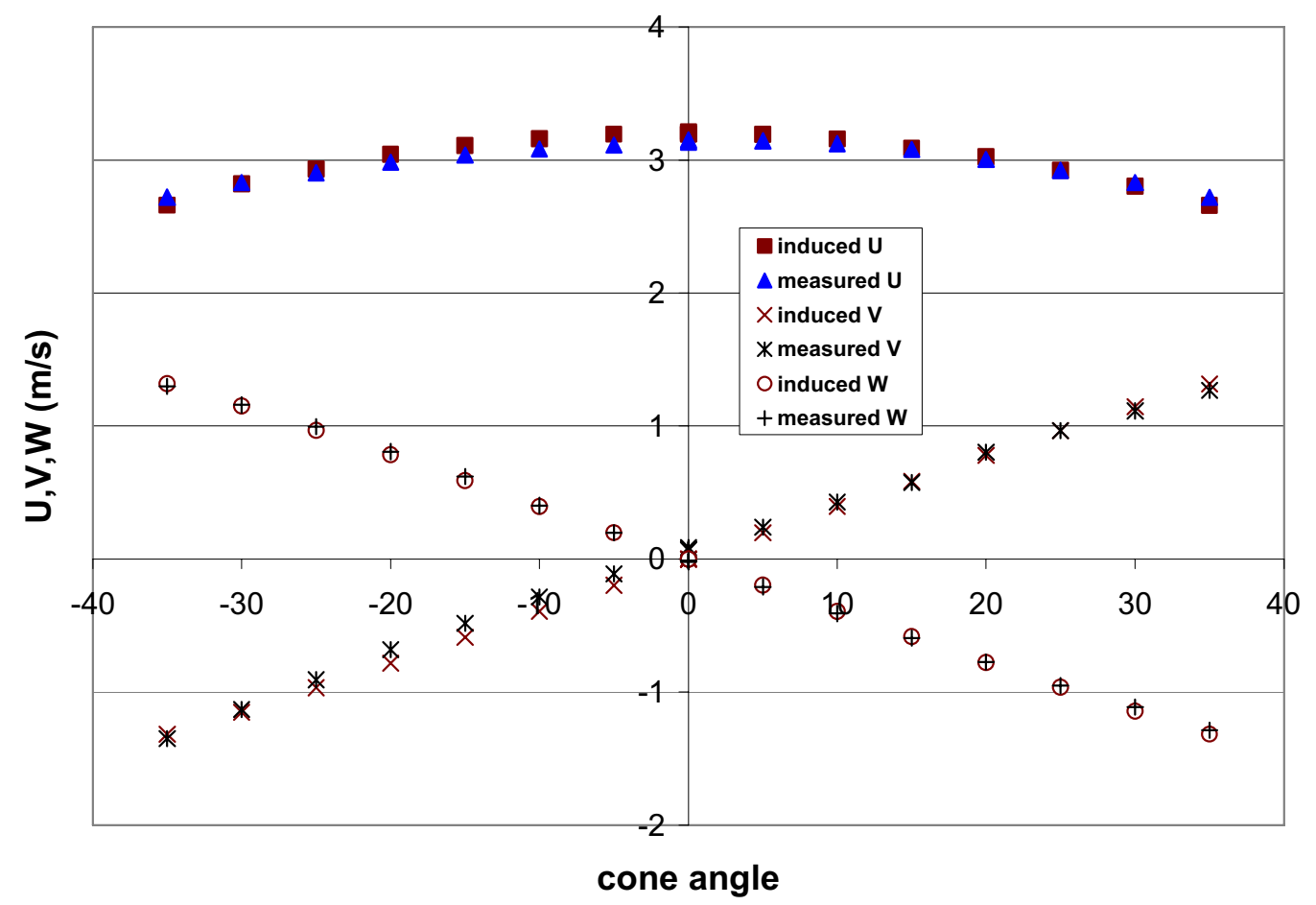

Fig. 6-15. The comparison of measured and induced velocity components at $80 \mathrm{C}$.

The accuracy at $80 \mathrm{C}$ is as good as that at room temperature. This result means that the mutual interference of sensors is of the same order as at room temperature and that the probe sensitivity is not affected significantly at elevated temperature.

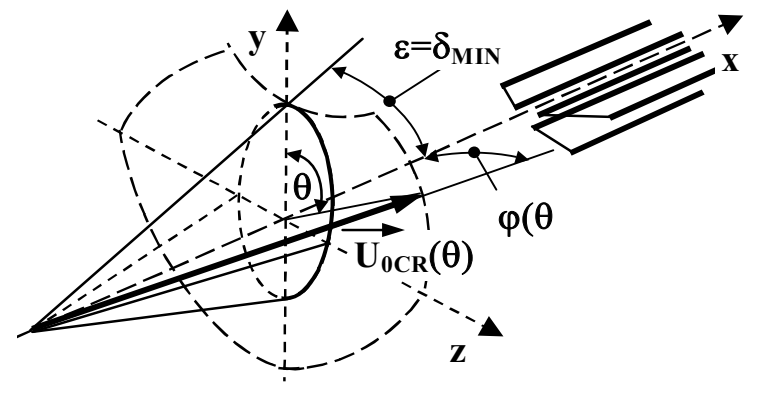

Fig. 6-16. The uniqueness domain and uniqueness cone of three-sensor hot-wire probe. five-sensor probe designed for this project is chosen as the one with the highest uniqueness range. The maximum theoretical value of the velocity vector angle, for which the velocity components can be uniquely determined, is about 40 degrees, very close to the sensor angle of 45 degrees. 
The experimental investigation of the uniqueness range, at a temperature of 80, is presented in Figure 6-17. Choosing three out of four available equations (6-3.1) and eliminating the $\mathrm{U}$ and $\mathrm{W}$ velocity components, one can determine a function $F(V)$. The intersection of this function with the abscissa determines the $\mathrm{V}$ velocity component. If it has one intersection, the solution is unique and vice-versa. It is obvious that, for a proper choice of the three sensor signals, the solution is unique up to 40 degrees (the upper solid lines). This observation means that even at elevated temperatures, the experimentally-determined uniqueness range is close to the theoretical one. For an optional choice of the available signals, two or more solutions can appear (the dotted lines), reducing the uniqueness range to about 30 degrees. This situation would be the case if only three sensors were available.

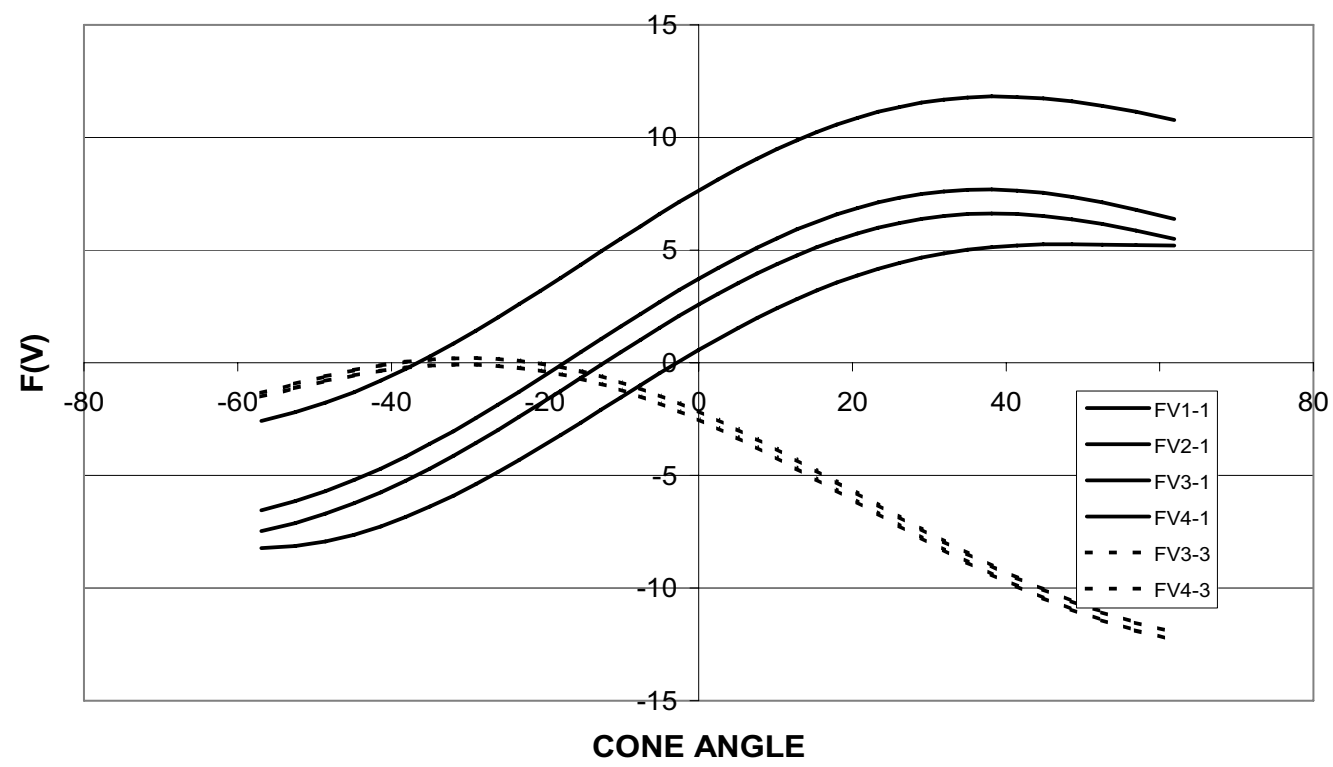

Fig. 6-17. Experimental determination of the uniqueness range.

Two-sensor probe in supercritical carbon dioxide flow. Little can be found in the literature about the response of a hot wire sensor in a fluid under conditions different from those at atmospheric pressure. Furthermore, there are hardly any data that can be used to describe the sensor response near the critical condition. This situation is due to the fact that fluids have complex physical and chemical properties that strongly depend on pressure and temperature in the region near the critical point. In addition, if a bare metal sensor is used for such measurements, the fluid should be free of any contaminant in order to reduce electrical conduction.

Following the well-known Newton's cooling law, one may express the convective heat flux, F, from a heated wire as

$$
F=h S\left(T_{w}-T_{f}\right)
$$

where $h$ is the convective heat transfer coefficient, $S$ is the wire surface area, $T_{w}$ is the wire temperature and $T_{f}$ is the temperature of the supercritical fluid. The coefficient of heat convection from the sensor to the surrounding flow field, $h$, can be expressed non-dimensionally by the Nusselt number, $N_{u}=h D_{w} / \lambda_{f}$, which is related to the Reynolds number, $R_{e}=U_{e} D_{w} / v_{f}$ and the Prandtl number, $P_{r}=v_{f} / \alpha_{f}$. Kramer's [1946] formula,

$$
N_{u}=C_{1} P_{r}^{0.2}+C_{2} P_{r}^{0.33} R_{e}^{0.5}
$$

has been shown to give reasonable results for water, ethylene glycol and some other liquids. The fluid characteristics are the thermal conductivity, $\lambda_{f}$, kinematic viscosity, $v_{f}$, and thermal diffusivity $\alpha_{f}$, determined for the film temperature $T_{r e f}$, and the Reynolds number, $R_{e}$, is based on $U_{e}$, the effective velocity cooling the sensor. The 
film temperature is defined as an arithmetic mean of the fluid and sensor temperatures. According to Blackwelder's [1981] analysis based on the results obtained by different authors, a more general formula

$$
N_{u}=M\left(P_{r}\right)+N\left(P_{r}\right) R_{e}^{n},
$$

can be used as a guide for the theoretical analysis. Heat transfer experiments for supercritical flow can be found only for internal flows. A survey of Nusselt number relations for this condition is given by Cheng and Schulenberg [2001]. They are similar to expressions (6-3.3) and (6-3.4) with different constants and an additional correction factor for the strong variation of the properties.

It thus appears that these formulas can only be used for a qualitative analysis in order to optimize the sensor parameters. For precise measurements, it is necessary to calibrate each sensor under the same conditions expected in a given turbulent flow, as is even true for fluids under normal conditions. It is expected that all problems related to heat transfer from the sensor to the surrounding fluid will become much more severe under supercritical conditions due to the extremely strong variation of the properties in the region around the pseudo-critical temperature.

The energy equation for a hot-wire sensor can be written as

$$
\frac{d U}{d t}=Q-F
$$

where $U$ is the internal energy of the sensor, $Q$ the thermal energy generated in the sensor by Joule's effect and $F$ is the energy transferred to the surroundings, mainly by convection heat flux. This equation can be rewritten with the aid of relations (6-3.2) and (6-3.4), as

$$
\frac{E_{w}^{2}}{R_{w}}=\lambda_{f} \pi L_{w}\left[M\left(P_{r}\right)+N\left(P_{r}\right) R_{e}^{n}\right]\left(T_{w}-T_{f}\right)+c_{w} \rho_{w} \frac{D_{w}^{2} \pi}{4} L_{w} \frac{d T_{w}}{d t},
$$

where $L_{w}, D_{w}, c_{w}$ and $\rho_{w}$ are the wire length, diameter, specific heat and density, respectively.

The velocity sensor is operated in the CTA mode with sensor temperature $T_{w}=$ const. It follows from equation (6-3.6) that for a given sensor temperature, $T_{w}$, the sensor output voltage $E_{w}$ will depend on the effective velocity $U_{e}$, the supercritical fluid temperature $T_{f}$, and the physical properties of the supercritical fluid. Keeping in mind that the fluid properties in the supercritical region strongly depend on pressure, $p$, and temperature, $T_{f}$, one may write expression (6-3.6), for a given sensor, in the general form,

$$
E_{w}^{2}=A\left(p, T_{f}\right)+B\left(p, T_{f}\right) U_{e}^{n}
$$

Along a line of constant pressure, the sensor output should have the form of King's "law", with parameters depending only on the supercritical fluid temperature,

$$
E_{w}^{2}=A\left(T_{f}\right)+B\left(T_{f}\right) U_{\mathrm{e}}^{\mathrm{n}}
$$

The dependence of parameters $A$ and $B$ on temperature $T_{f}$ must be found experimentally as will be shown below.

Our experiments were performed at constant pressure of 80 bar with different wire temperatures. The supercritical fluid temperature was varied from 20 to $60 \mathrm{C}$ with variable steps. At each temperature step, the speed was varied while keeping the pressure and temperature constant and the sensor output $E_{w}$ was recorded. The results are shown in Figure 6-18. 


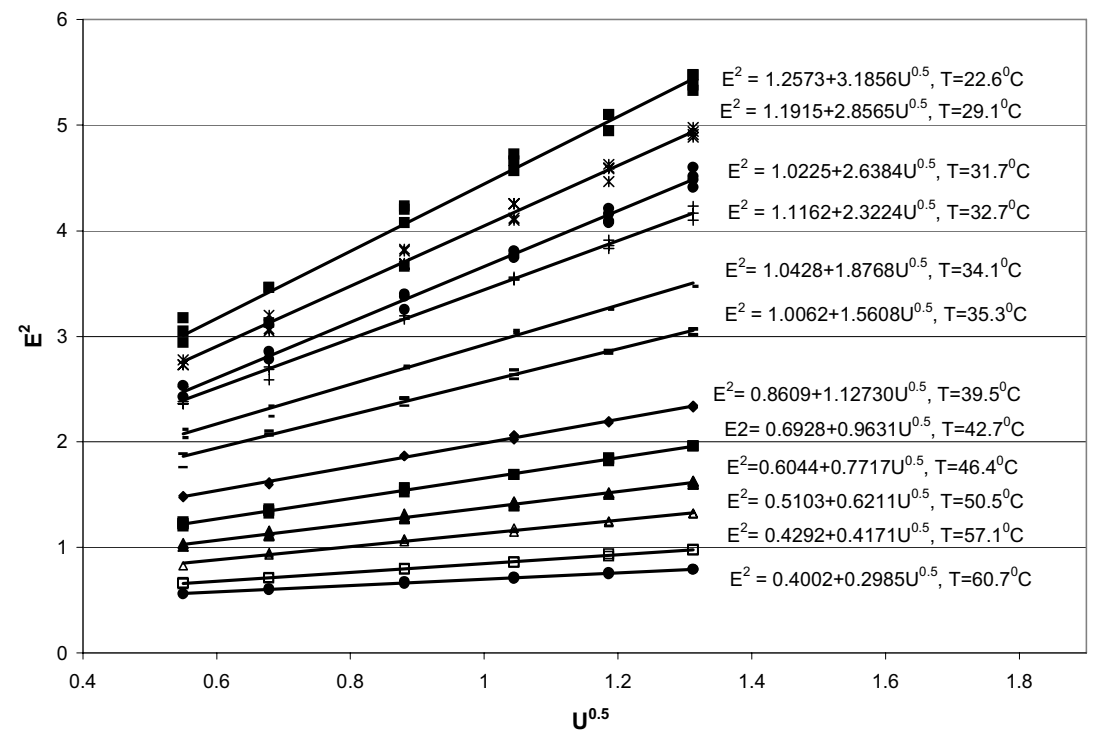

Fig. 6-18. Sensor voltage output at different speeds and temperatures and at constant pressure ( $80 \mathrm{bar})$.

As the discussion above suggested, the data fit King's law (6-3.8) with a high correlation, but only above the pseudo-critical temperature $\left(T_{p s c}=34.7 \mathrm{C}\right.$ at $\left.\mathrm{p}=80 \mathrm{bar}\right)$. Considerable data scatter is present below the pseudocritical temperature and it is not completely stochastic. In most cases, for each speed variation but with the same pressure and temperature, the data follows King's law well below the pseudo-critical temperature; however, each King's law fit is different, as can be seen in Figure 6-19.

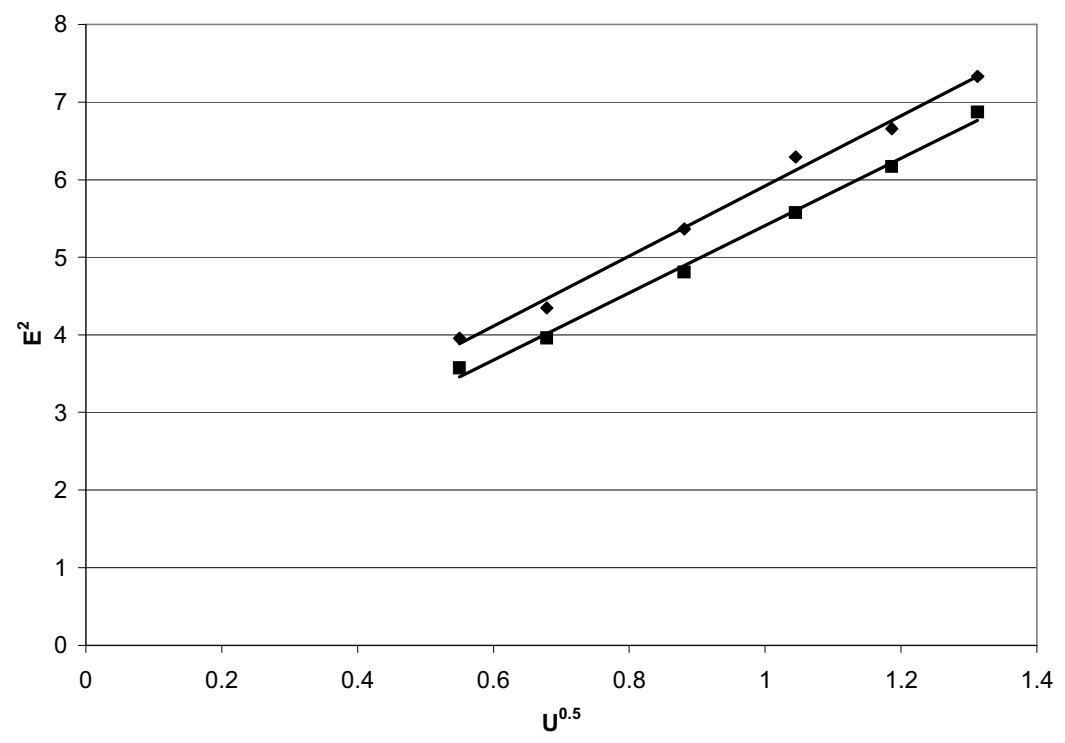

Figure 6-19. Example of sensor response below the pseudocritical temperature at constant pressure ( $80 \mathrm{bar})$ and temperature: $\bullet$, increasing speed; $\boldsymbol{\square}$, decreasing speed.

Rarely does a shift occur during a single speed variation. There were also rare cases when the shift occurred up and down several times during one single set of speed variations. As can be seen in Figure 6-19, the common characteristics of these cases are that parameter B is practically the same if the shift does not occur during a single speed variation, while different values of parameter A are obtained. If the shift occurs during a single speed 
variation, both parameters A and B will be affected. If the number of data points is sufficient, an average value of B will not be affected, because the shift does not always occur at the same position. Sometimes it happens that only one sensor is affected even when two sensors are running simultaneously at the same overheat ratios.

The dependencies of $A$ and $B$ on temperature are presented in Figures 6-20(a) and (b), respectively. In order to test the influence of the sensor temperature on the stability of the sensor response, two sensors, with different overheat ratios, were run simultaneously.

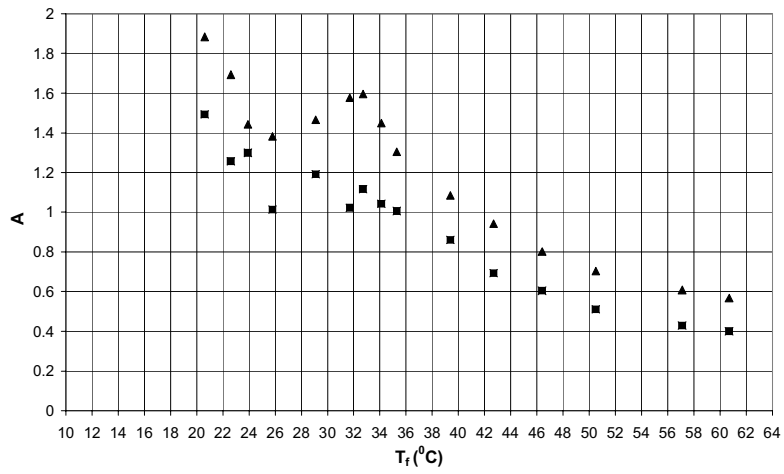

(a)

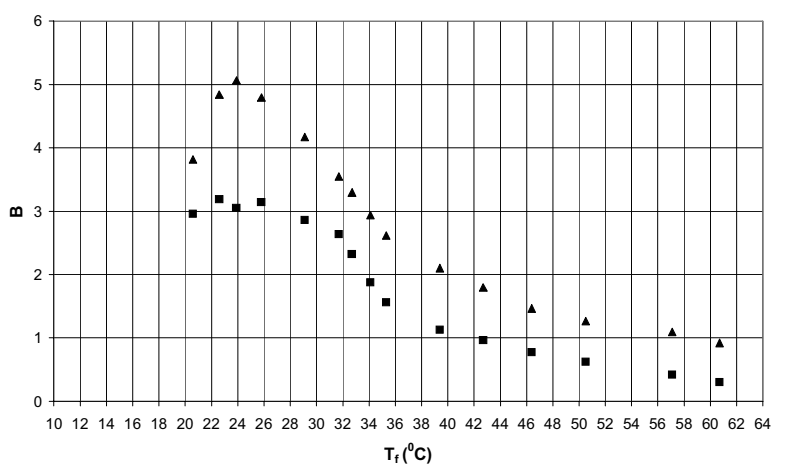

(b)

Fig. 6-20. Variation of King's law parameters $\mathrm{A}$ and $\mathrm{B}$ with fluid temperature: (a) parameter $A$, (b) parameter $B$; $\mathrm{T}_{\mathrm{w}}=65^{\circ} \mathrm{C} ; \boldsymbol{\Delta} \mathrm{T}_{\mathrm{w}}=85^{\circ} \mathrm{C}$.

As is evident from these figures, above the pseudo-critical temperature $\left(\mathrm{T}_{\mathrm{pc}} \approx 34.7 \mathrm{C}\right)$, the dependence of $A$ and $B$ on fluid temperature can be accurately approximated with a second-order polynomial or exponential fit, depending on the extent of the fluid temperature range. In this region, the data are repeatable, not only from one speed variation run to another, but also for two or more different charges of $\mathrm{CO}_{2}$. Below the pseudo-critical temperature, however, the variation of $A$ and $B$ with fluid temperature is irregular. As can be expected due to the shifts of sensor responses presented in Figure 6-19, parameter $A$ is affected much more than parameter $B$. This irregularity, as well as the data scatter shown in Figure 6-18 for this temperature range, cannot be explained by the strong variation of fluid properties alone. The magnitude of the property variation just below and just above pseudocritical temperature is of the same order. The difference is that in the case of $T_{f o c}<T_{p s c}$ the maximum value of the Prandtl number occurs inside the sensor's thermal boundary layer, while in the case of $T_{f o}>T_{p s c}$ it must be outside this thermal boundary layer, as illustrated in Figure 6-21. 


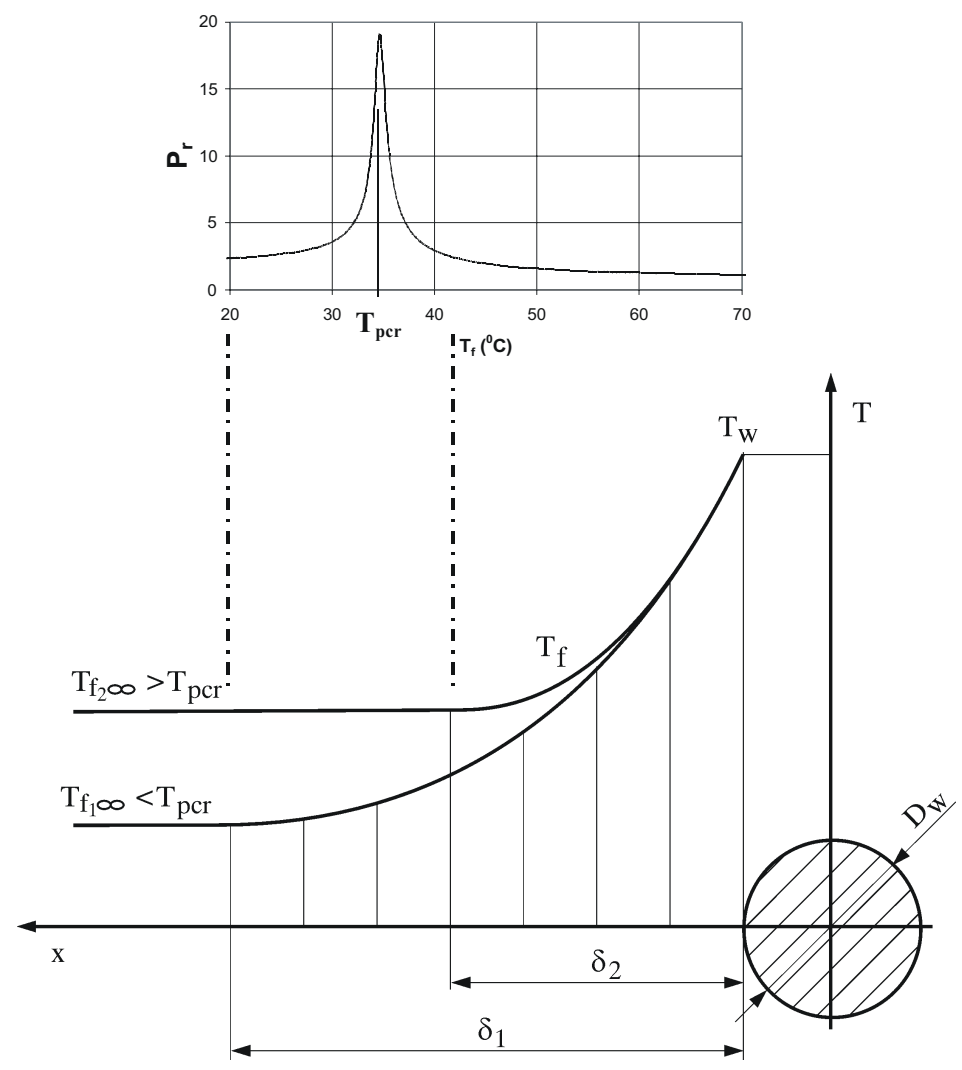

Fig. 6-21. Schematic view of the sensor thermal boundary layer with related Prandtl number variation.

For $T_{f \infty}>T_{p s c}$ the supercritical fluid properties change continuously from the value outside the boundary layer to the value that corresponds to the wire wall temperature $T_{w}$. For $T_{f \infty}<T_{p s c}$ an extreme of the Prandtl number will occur inside the thermal boundary layer causing strong property variations. The position of this maximum probably oscillates depending on the turbulence level as well as on any speed instabilities present in the flow, thereby changing the fluid property field in the sensor's vicinity. This situation will affect the heat transfer condition around the sensor and therefore the value of parameters $A$ and $B$.

A similar problem has been found in the case of supercritical pipe flows. It is referred to as heat transfer "deterioration" and is characterized by a decrease of the heat transfer coefficient and an increase of the wall temperature. It has been difficult to define the onset of this phenomenon, but it always happens in the region of $T_{B} \leq T_{p s c} \leq T_{w}$, for pipe flow, where $T_{B}$ is the fluid bulk temperature [Jackson, Cotton and Axcell, 1989]. This occurrence is equivalent to the case of $T_{f \circ o} \leq T_{p s c} \leq T_{w}$, shown in Figure 6-21, for the flow around a cylinder. Different relationships are used to predict this phenomenon in pipe flows. Some are based on the ratio of the heat transfer coefficient to a reference value defined experimentally.

If the sensor temperature is increased relative to the fluid temperature, the extreme values of the fluid properties will occur farther from the sensor and vice versa. The farther the extreme values of the fluid properties are located from the sensor wall temperature, the smaller is their expected influence on the heat exchange condition. This observation means that the sensor temperature could be one of the parameters to be optimized in order to improve the sensor response below the pseudo-critical temperature. It is evident from Figure 6-20(a) that the scatter of parameter $A$ data is smaller for the case of a higher sensor temperature $T_{w}$. The data scatter below the pseudocritical temperature was also smaller. This result is preliminary. The heat transfer "deterioration" is a very complex problem and it will take considerable effort to optimize the sensor response in the range where this phenomenon can occur. 
The temperature sensor is operated in the CCA mode, behaving as a resistance thermometer. The wire voltage $E_{w}$ is sensitive to any variation of parameters relevant to heat exchange between the sensor wire and its environment. Taking into account that the wire resistance $R_{w}$ varies linearly with temperature over a temperature range that is not too great, i.e.

$$
R_{w}=R_{0}\left[1+\alpha\left(T_{w}-T_{0}\right)\right]
$$

where the subscript o denotes a reference temperature and resistance, one may rewrite expression (6-3.6) as

$$
\Phi \frac{d R_{w}}{d T}+R_{w}=\varphi(t)
$$

For a sufficiently small current through the sensor,

$$
\Phi=\frac{\rho_{w} c_{w} D_{w}^{2}}{4 \lambda_{f}\left(M+N R_{e}^{n}\right)} \text { and } \varphi(t)=R_{0}\left[1+\alpha\left(T_{f}-T_{0}\right)\right]
$$

It is obvious that $\Phi$ is the time constant of the sensor's response to the forcing function $\varphi(t)$. For a sufficiently small time constant $\Phi$, which can be achieved using a sensor with small diameter $D_{w}$, relation (6-3.10) reduces to the simpler form $T_{w}=T_{f}$, which means that the sensor temperature is equal to the supercritical fluid temperature. This result is equivalent to a linear function between the anemometer output $E_{w}$ and the supercritical fluid temperature $T_{f}$, i.e.,

$$
E_{w}=k_{0}+k_{1} T_{\mathrm{f}}
$$

Expression (6-3.12) is the same as for the heated air flow, as discussed by Vukoslavčević and Wallace [2002]. The constants $k_{0}$ and $k_{1}$, which should not depend on speed, pressure and temperature, can be determined experimentally by a calibration procedure. For the temperature in the range from $20 \mathrm{C}$ to $60 \mathrm{C}$ and with variable speed and pressure, the linear relationship given by equation (6-3.12) is demonstrated in Figure 6-22.

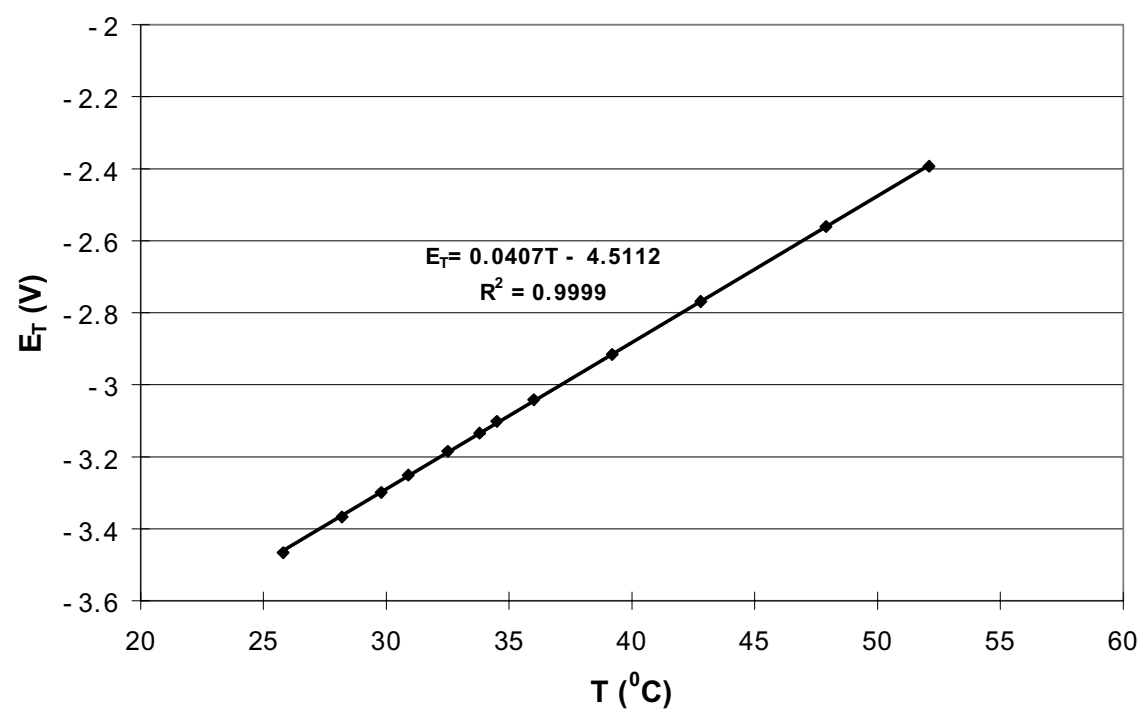

Fig. 6-22. Response of the temperature sensor. 
In contrast to the velocity sensor response, the temperature sensor response is not affected by the heat deterioration phenomenon in the pseudocritical region. To check whether the temperature sensor is affected by speed, it was varied at constant temperature and pressure, and the sensor output was recorded. As can be seen in Figure 6-23, the sensor is insensitive to speed variation. All of these results have been reported by Vukoslavčević, Radulović and Wallace [2005].

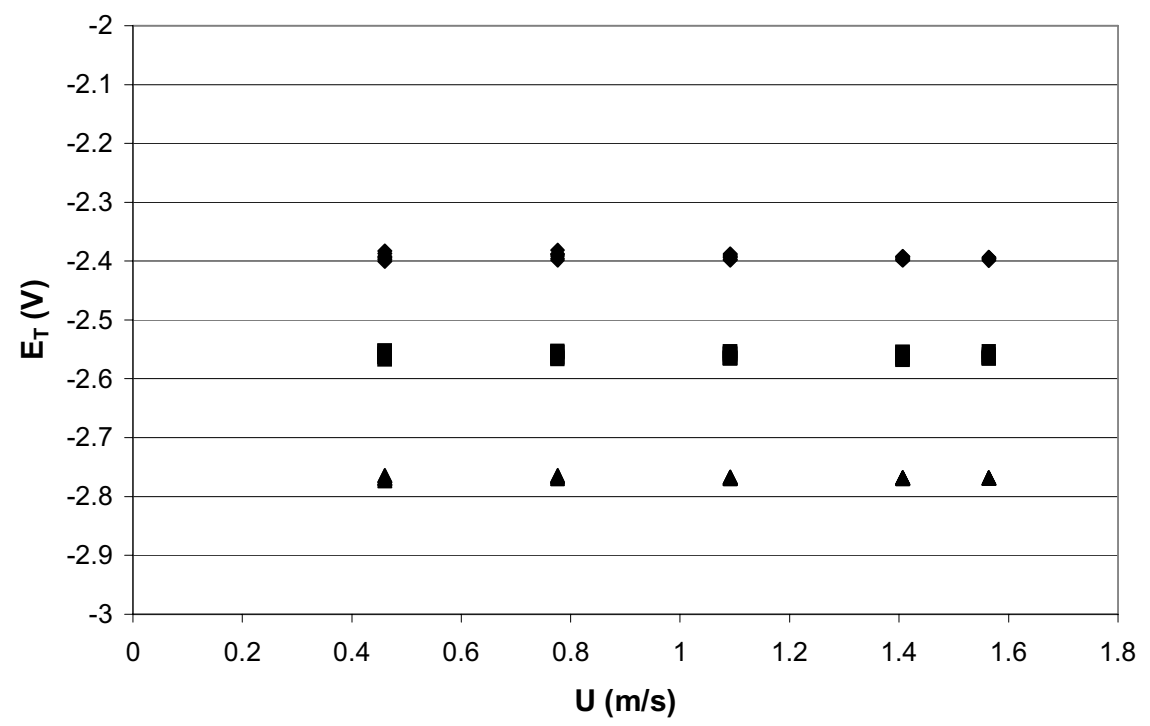

Fig. 6-23. Response of temperature sensor at constant temperature and pressure and variable speed:

$\bullet \mathrm{T}=52 \mathrm{C}, \boldsymbol{\mathrm { T }}=47.8 \mathrm{C}, \boldsymbol{\Delta} \mathrm{T}=42.8 \mathrm{C}$.

\section{6-4. Data Analysis Algorithm}

Five-sensor probe. By appropriate choice of three of four available equations (6-3.1), the velocity components $\mathrm{U}, \mathrm{V}$ and $\mathrm{W}$ can be determined. The equations are nonlinear, so a numerical approach is necessary to solve them. The uniqueness range as well as the measurement accuracy depends on the optimal choice of the available signals. The procedure used by Vukoslavčević and Wallace [1996] for a 12-sensor probe can be applied for velocity measurements with a five-sensor probe. The temperature measurements with cold sensor of five-sensor probe are the same as they were with the cold sensor of the three-sensor probe described by Vukoslavčević and Wallace [2002].

Two-sensor probe. In the measurement procedure with this probe in supercritical flow conditions, the temperature has to be determined first from relation (6-3.12), then parameters $A$ and $B$ can be obtained from the graphs presented in Figure 6-20 or by fits to the data, and finally the speed from expression (6-3.8). If the time constant $\Phi$ is not small enough, i.e., the temperature fluctuation frequency is at or above a critical value defined by $f_{c}=1 / 2 \pi \Phi$, an iterative approach should be applied in order to determine the dependence of $\Phi$ on speed and temperature. The temperature can be determined in a first approximation from relation (6-3.12), then the speed from relation (6-3.8), and finally the time constant $\Phi$. The second and higher temperature iterations follow from relation (6-3.10), which can be rewritten in the form

$$
\Phi \frac{d E_{w}}{d t}+E_{w}=k_{0}+k_{1} T_{\mathrm{f}}
$$

Analysis of heat transfer mechanism around a circular cylinder. The two sensor probe was designed and tested to be used for velocity and temperature field measurements in a vertical pipe flow experiment designed at the Seoul National University. Unfortunately, the preparation of the experiment was not completed in time for this 
type of measurement. With this in mind, we decided to analyze the heat transfer mechanism around a circular cylinder in supercritical flow; it was possible to perform this study in our installation that had been developed to test and calibrate the probe for supercritical flow measurements. Although, from a practical point of view, the heat transfer mechanism around a circular cylinder is not as important as the heat transfer in a circular pipe, we believe that obtaining more knowledge about the physics of this process can contribute to a clearer understanding of the physics of the heat transfer mechanism in pipe flows. The existing facility, developed to calibrate the hot-wire sensors in supercritical $\mathrm{CO}_{2}$ flow, is very convenient for this analysis. It is capable of creating variable speed flow at different supercritical conditions. The hot-wire sensor has the shape of circular cylinder. By appropriate choice of sensor dimensions and temperature, various flows around a circular cylinder can be created and controlled.

We performed our experiments at 80 bar and various fluid temperatures in the range from $29 \mathrm{C}$ to $65 \mathrm{C}$ in steps from 0.5 to $5 \mathrm{C}$. At each temperature step the speed was varied in the range from 0.3 to $2 \mathrm{~m} / \mathrm{s}$. Based on our experience related to the stability of hot-wire sensor response, the cylinder temperature was chosen to be $86 \mathrm{C}$.

All parameters needed to determine the Nusselt and Reynolds numbers, i.e., flow speed, fluid properties and thermal energy generated and transfer by convection from the cylinders, can be measured precisely. One of the main parameters to be chosen for this analysis was the reference temperature to evaluate the fluid properties. There is no unique approach to this problem. Frequently the film temperature, $\mathrm{T}_{\text {film }}$ (equal to the mean of the fluid and wall temperatures) or the fluid temperature at an infinite distance from the wall, $\mathrm{T}_{\text {inf }}$, is used to evaluate the fluid properties. Keeping in mind the very strong variations of supercritical $\mathrm{CO}_{2}$ properties with temperature (e.g., $\operatorname{Pr}\{\mathrm{T}\}$ in Figure 6-24), we tested both approaches in this analysis.

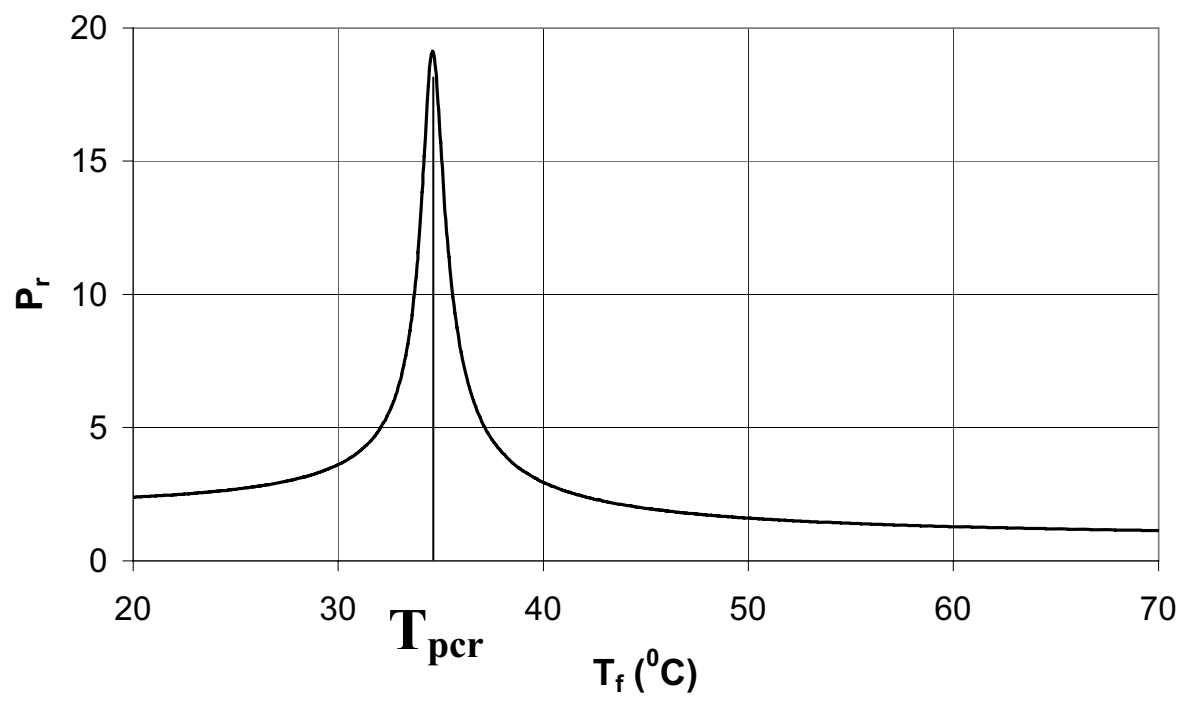

Fig. 6-24. The variation of Prandtl number with fluid temperature around the pseudocritical point $\mathrm{T}_{\mathrm{pcr}}$, at 80 bar pressure.

An example of $\mathrm{Nu}=\mathrm{F}(\mathrm{Re})$ number, at fluid temperature $\mathrm{T}_{\text {inf }}=35.1 \mathrm{C}$ and cylinder temperature $\mathrm{T}_{\mathrm{c}}=86 \mathrm{C}$ is given in Figure 6-25. The fluid properties were evaluated at the fluid temperature $\mathrm{T}_{\text {inf. }}$ Similar results were obtained for the fluid properties evaluated at the film temperature $\mathrm{T}_{\text {film. }}$. The relation $\mathrm{Nu}=\mathrm{F}(\mathrm{Re})$ has the form $N_{u}=M+N R_{e}^{n}$, which is characteristic for most known cases of gas and liquids flows around circular cylinders. For these cases, parameters $\mathrm{M}$ and $\mathrm{N}$ are proportional to Prandtl number in powers $\mathrm{m}_{1}$ and $\mathrm{m}_{2}$. They are practically constant for gas flows over a wide temperature range due to of the small variation of the Pr with temperature. 


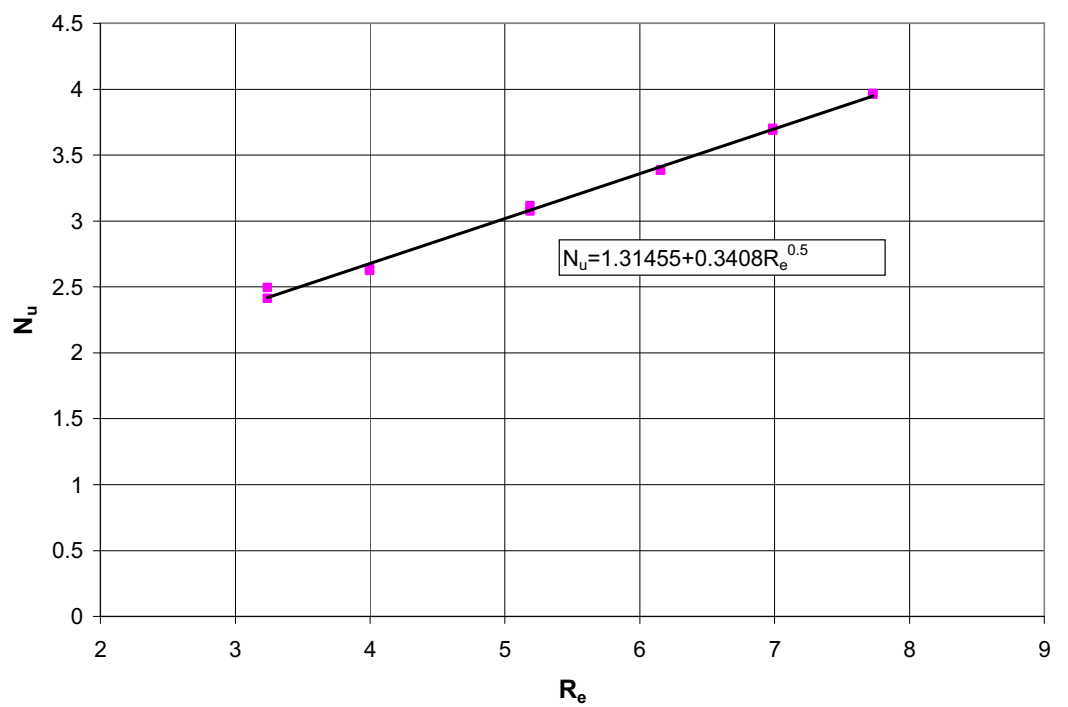

Fig, 6-25. The relation $\mathrm{Nu}=\mathrm{F}(\mathrm{Re})$ at $\mathrm{T}_{\text {inf }}=35.1 \mathrm{C}$ and $\mathrm{T}_{\mathrm{c}}=86 \mathrm{C}$, with fluid properties evaluated at $\mathrm{T}_{\text {inf. }}$.

With the strong variation of $\operatorname{Pr}\{\mathrm{T}\}$ for supercritical fluids around the pseudocritical in mind, as shown in Figure 6-24, we focused our analysis on the dependence of parameters A and B on $\operatorname{Pr}$ in this region. In order to choose the most appropriate reference temperature, the variations of the parameters $\mathrm{M}$ and $\mathrm{N}$ with temperature are presented for two different cases. For the case shown in Figure 6-26, the fluid properties are evaluated at the film temperature, $\mathrm{T}_{\text {film. }}$. For the case shown in Figure 27, the properties were evaluated at the fluid temperature infinitely far from the wall, $\mathrm{T}_{\mathrm{inf}}$.

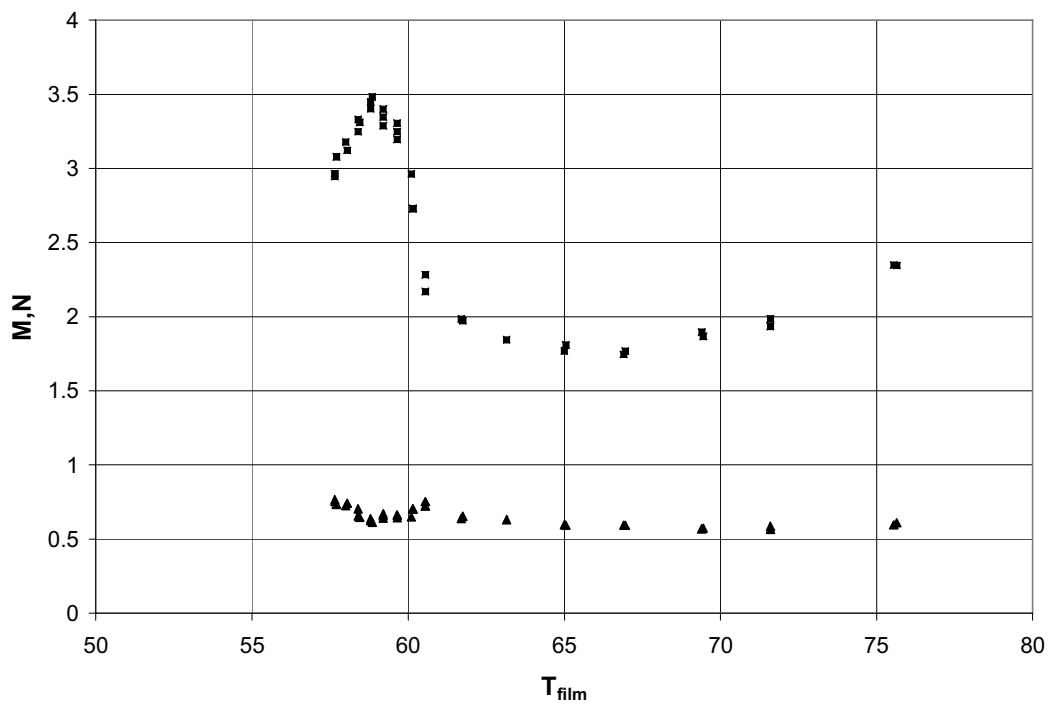

Fig. 6-26. The dependence of parameters $\mathrm{M}(\boldsymbol{\bullet})$ and $\mathrm{N}(\boldsymbol{\Delta})$ on temperature for the fluid property evaluated at $\mathrm{T}_{\text {film}}$. 


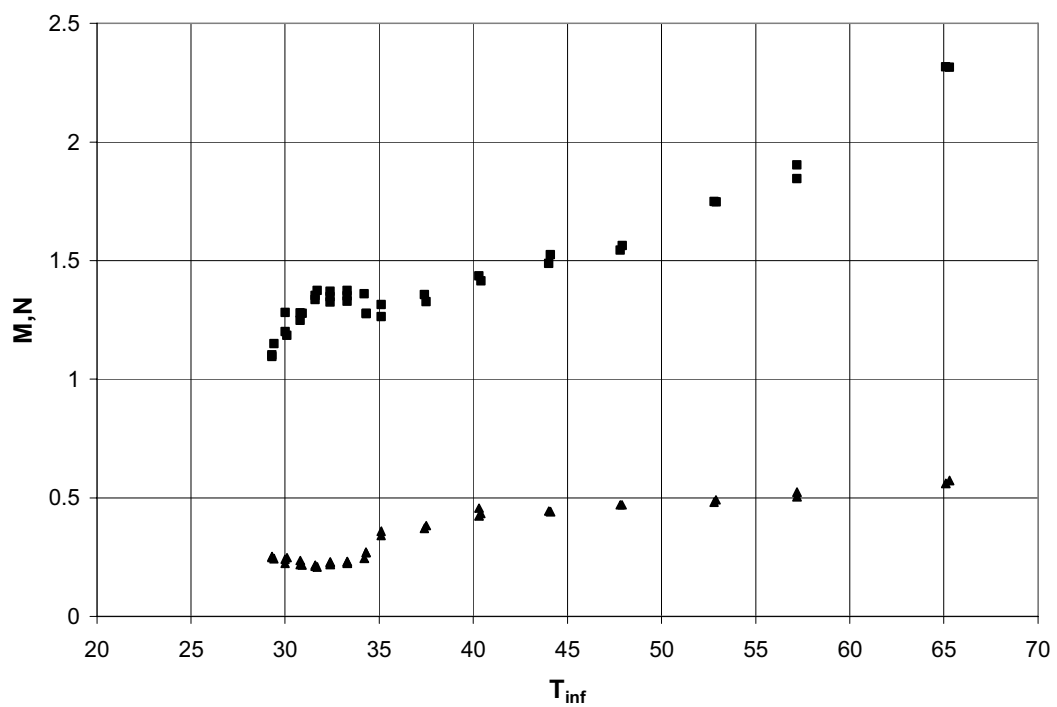

Fig. 6-27. The dependence of parameters $\mathrm{M}(\mathbf{-})$ and $\mathrm{N}(\boldsymbol{\Delta})$ on temperature for the fluid property evaluated at $\mathrm{T}_{\text {inf. }}$.

It is clear from Figure 6-27 that the variations of parameters $\mathrm{M}$ and $\mathrm{N}$ with temperature are in accordance with the variation of $\mathrm{Pr}$ with temperature. There is a maximum around the pseudocritical and a smooth variation above this point. This is not the case for $\mathrm{M}$ and $\mathrm{N}$ evaluated at $\mathrm{T}_{\text {film }}$, as shown in Figure 6-26. Although parameter $\mathrm{M}$ has a minimum around $67 \mathrm{C}$, the Pr number does not have any extreme in this region. So, we believe that the most appropriate temperature to evaluate fluid properties is the fluid temperature far from the cylinder, $\mathrm{T}_{\mathrm{inf}}$, or a temperature close to $\mathrm{T}_{\mathrm{inf}}$.

Another experiment was performed along at 80 bar covering the lower temperature range of 25-60 C. Two cylinders were examined simultaneously with aspect ratios $1_{1} / \mathrm{d}=340$ and $\mathrm{l}_{2} / \mathrm{d}=260$. The fluid properties were evaluated at $\mathrm{T}_{\text {inf. }}$. The parameters $\mathrm{M}$ and $\mathrm{N}$ are presented as a function of Prandtl number. Unfortunately, due to the non unique values of $\mathrm{P}_{\mathrm{r}}$ number in the pseudocritical region (the same value of $\mathrm{P}_{\mathrm{r}}$ corresponds to two different fluid temperatures), the presentation of $\mathrm{M}$ and $\mathrm{N}$ as a function of $\mathrm{P}_{\mathrm{r}}$ number does not seem to be convenient, as can be seen from Figure 6-28.

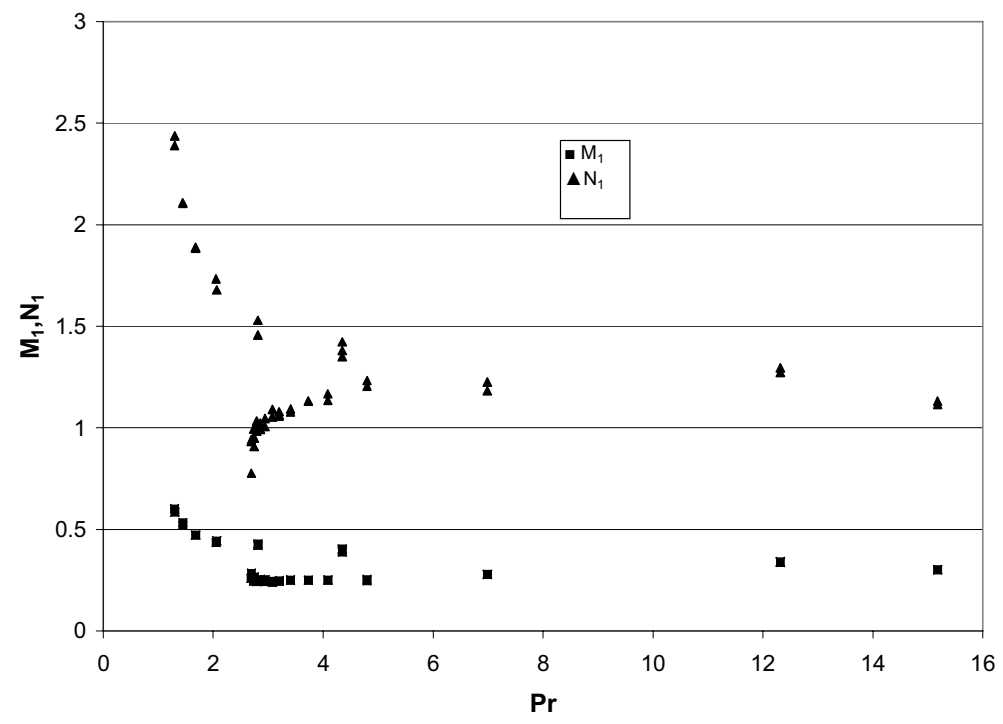

Fig. 6-28. The dependence of parameters $\mathrm{M}$ and $\mathrm{N}$ on $\mathrm{P}_{\mathrm{r}}$ number for sensor 1, fluid properties evaluated at $\mathrm{T}_{\text {inf. }}$. 
For the same value of $\mathrm{P}_{\mathrm{r}} \mathrm{r}$ two different values of $\mathrm{M}$ and $\mathrm{N}$ are possible. Another reason to avoid the presentation of $\mathrm{M}$ and $\mathrm{N}$ in terms of $\mathrm{P}_{\mathrm{r}}$ is the very strong $\mathrm{P}_{\mathrm{r}}$ variation with temperature around the pseudocritical temperature. It is clear that a conventional form $M=C_{1} P_{r}^{n 1}$ and $N=C_{2} P_{r}^{n 2}$, cannot be found in this case. The presentation of $\mathrm{M}$ and $\mathrm{N}$ as functions of fluid temperature seems to be much more convenient, as can be seen in Figure 6-29. The parameter $\mathrm{N}$ is almost constant in the range below $\mathrm{T}_{\mathrm{pc}}$ and it has a slight linear variation above it. The parameter $\mathrm{M}$ is practically linear for both ranges with different slopes. There is some scatter of the data below the pseudocritical temperature due to the instability in this region.

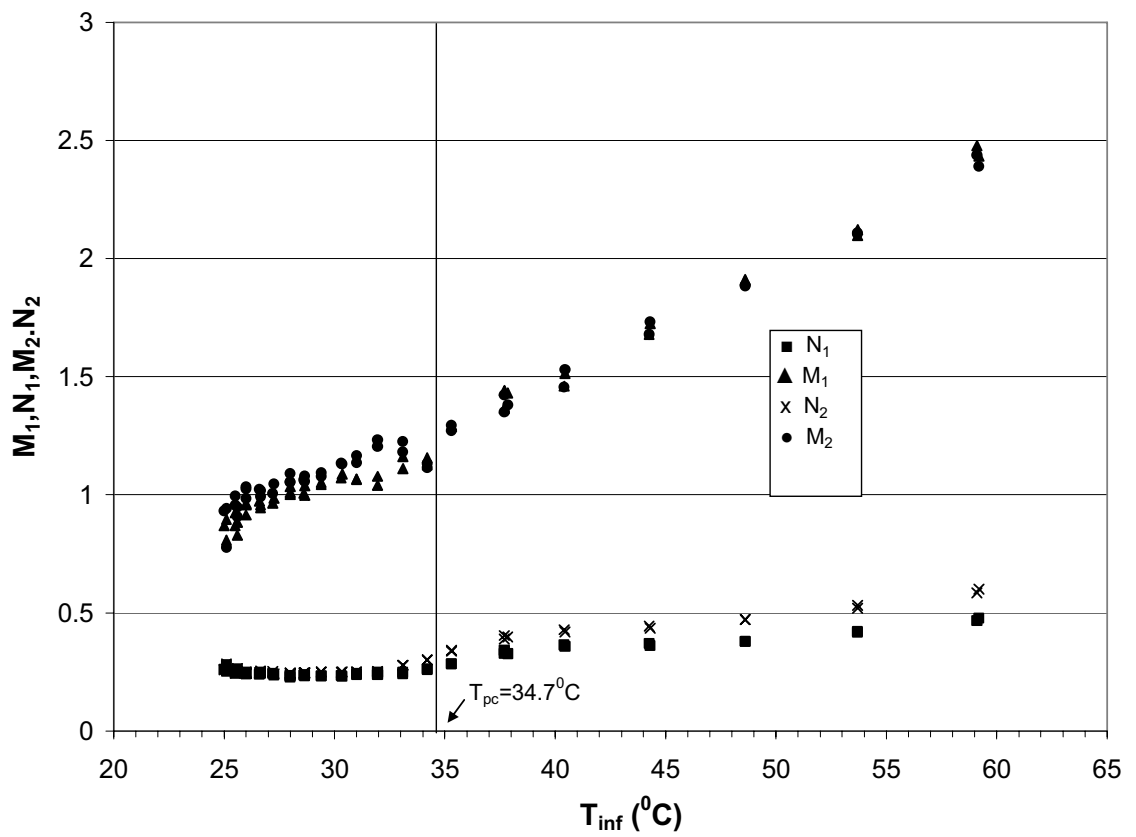

Fig. 6-29. Parameters $\mathrm{M}$ and $\mathrm{N}$ for both sensors.

The parameters $\mathrm{M}$ and $\mathrm{N}$ should be the same for both sensors (they should not depend on the sensor's length). It can be seen from Figure 6-29 that the parameter $M$ is practically the same.(In Figures 6-25 through 6-29 the exponent $\mathrm{n}$ has been taken to be $1 / 2$.) However, while parameter $\mathrm{N}$ is the same for the temperature range below $\mathrm{T}_{\mathrm{pc}}$, it is slightly different for the higher temperature above $\mathrm{T}_{\mathrm{pc}}$. The reason for this difference is not yet clear. Preliminary analysis does not suggest that it could be due to the support cooling effect. 


\section{Task 7. Heat transfer experiments - measurements of turbulent quantities in superheated and}

supercritical flow, Profs. J. S. Lee, S. T. Ro and J. Y. Yoo, Seoul National Univ. (SNU)

In developing turbulence models for supercritical flows, measurement data for basic turbulence quantities such as temperature and velocity distributions, turbulence kinetic energy, Reynolds stresses, etc. are requested for code validation. These data have not been available for strongly-heated internal flow of superheated and supercritical fluids. Experiments have been conducted at Seoul National University (SNU) focusing on the effects of property variations near the critical point as in the reactor core of proposed SCWR concepts.

The general goal is to obtain better understanding of flow and heat transfer characteristics in strongly heated, internal, turbulent superheated and supercritical flows, with an emphasis on turbulence structure. Hence, objectives are to measure the fundamental turbulence structure and to obtain benchmark data to assess CTFD codes at conditions in the range of ALWRs and SCRs. Conceptually, DNS codes, as by Satake et al. [2000] or You, Yoo and Choi [2003], could accomplish these aims and more; however, DNS calculations must also be compared to timeresolved experimental measurements to insure adequate spatial and temporal resolutions, to uncover coding mistakes, to check for prediction of measurable quantities and consistent, useful definitions of those quantities, etc.

Prof. Lee and colleagues at SNU have conducted several experiments for heat transfer of superheated and supercritical flows in vertical tubes. First, the test facility was established for experiments. Second, heat transfer experiments were conducted by using four different tubes to measure the wall temperature distributions and to compare the heat transfer phenomena appearing in circular and non-circular tubes. Finally, a hot wire traverse device and calibration loop were manufactured to measure the velocity, temperature and turbulent quantities of supercritical carbon dioxide flow.

Test facility The test facility is made of SUS 304 and the size of main pipe is $19 \mathrm{~mm}$ ( $3 / 4 \mathrm{inch})$. The design pressure of the facility is $10 \mathrm{MPa}$. Therefore, hydraulic pressure test was performed at $15 \mathrm{MPa}$ successfully. The schematic of test facility and photograph are shown in Figures 7-1 and 7-2 respectively. The test facility is composed of a main loop for carbon-dioxide and a secondary loop for cooling water. The working fluid is carbondioxide with $99.5 \%$ purity; it is pressurized up to $8 \mathrm{MPa}$ or higher by using an air-driven booster pump and an accumulator. The working fluid is pumped around the main loop by a magnetic gear pump. The mass flow rate is controlled by changing the pump rpm and is measured with a Coriolis type flowmeter. For the low mass flow rate test, another gear pump suitable for low rpm operation is also used. The carbon-dioxide is delivered to the electric preheater and/or to the bypass line when it is necessary to adjust the flow rate or stabilize the system. The electric preheater which has a capacity of $10 \mathrm{~kW}$ allows the fluid temperature to rise up automatically to a desired temperature at the inlet of the test section as listed in Table 7-1. After being heated above the critical point in the test section, the fluid cools to a temperature lower than the inlet temperature when it passes through two shell-and-tube type coolers. Cooler-1 and cooler-2 have heat transfer capacities of $39 \mathrm{~kW}$ and $19 \mathrm{~kW}$, respectively. The chiller is used for supplying city or chilled water to the two coolers with an appropriate flow rate and temperature. After circulating through the two coolers, the cooling water may be drained.

Table 7-1: Experimental conditions.

$\begin{array}{cc}\text { Pressure }[\mathrm{MPa}] & 8 \\ \text { Inlet fluid temperature }\left[{ }^{\circ} \mathrm{C}\right] & 15,25,32 \\ \text { Heat flux }\left[\mathrm{kW} / \mathrm{m}^{2}\right] & 3-180 \\ \text { Mass velocity (mass flux) }\left[\mathrm{kg} / \mathrm{m}^{2} \mathrm{~s}\right] & 209-1230\end{array}$



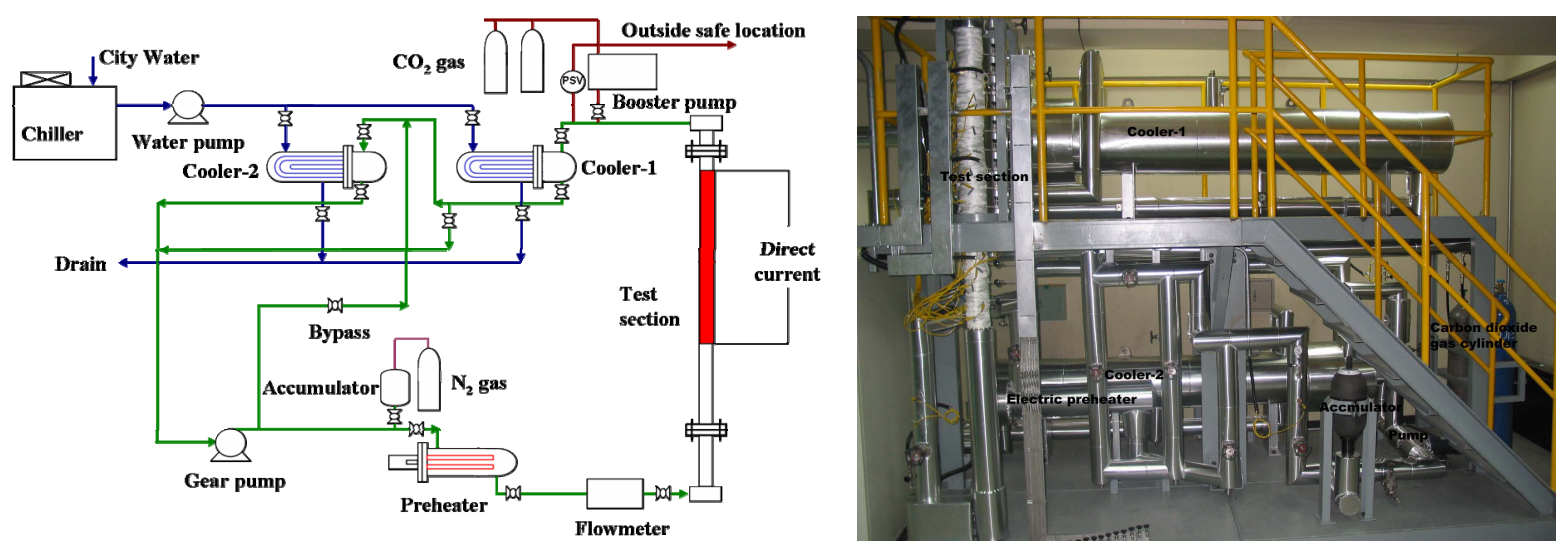

Fig. 7-1. Schematic diagram of test facility.

Fig. 7-2. Photograph of test facility.

Test section

To represent the heat transfer between a fuel rod and cooling water in a nuclear reactor core, the test section is vertically installed as shown in Figure 7-3. Four test sections with three different crosssection shapes are used as the test section. The four tubes are made of Inconel 625 and their dimensions are listed in Table 7-2 Small circular, triangular and square test sections are composed of entrance regions 75 diameters long and heating region 130 diameters in length (based on the small circular tube diameter). For the large circular tube, the entrance length is thirty diameters.

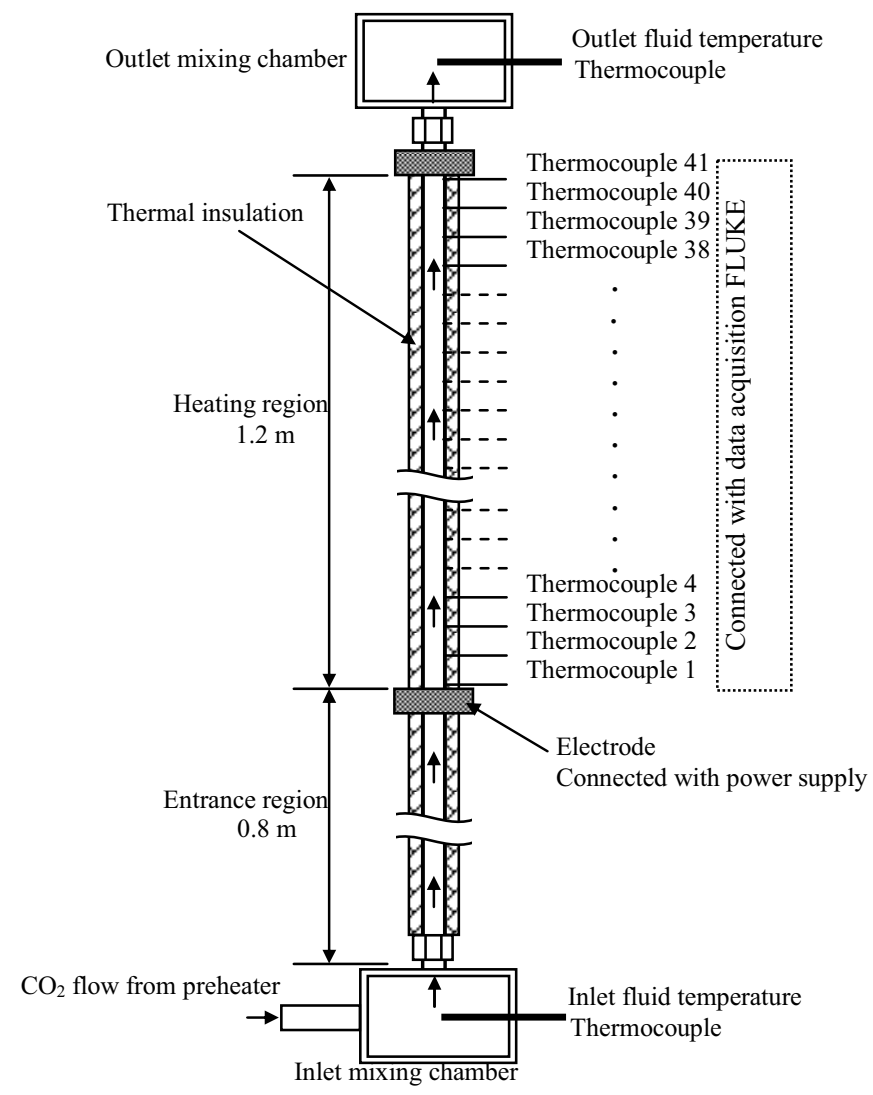

Fig. 7-3. Schematic diagram of test section based on the small circular tube.

The test tubes are vertically mounted to generate "aiding" mixed convection flows. To provide uniform heat flux at the heating region in the test sections, the DC (direct current) heating method is adapted. Electricity is 
supplied to the test section by using DC power unit which has a capacity of twenty volts and 450 amperes. The available maximum heat fluxes to the small circular, triangular and square tubes are 220,130 and $220 \mathrm{~kW} / \mathrm{m}^{2}$, respectively. In order to achieve electric insulation from the test section, sheath type thermocouples, insulation and teflon gaskets are used. Teflon gaskets are also used to seal the high pressure at the flanges. A sealing test was successfully performed at $10 \mathrm{MPa}$. The outer wall temperatures along the test tubes are measured with K-type (chromel-alumel) sheath type thermocouples. The sheath type thermocouple is electrically insulated. Forty-five thermocouples are silver soldered to the small circular, triangular and square tubes wall every thirty $\mathrm{mm}$ and thirtyfive thermocouples are also soldered at the large circular tube wall every forty $\mathrm{mm}$ from the starting point of the heating region. To confirm the uniformity in temperature around the circumference of the non-circular tubes, twenty thermocouples are additionally soldered at the corners every sixty $\mathrm{mm}$, and ten thermocouples every $120 \mathrm{~mm}$ part wat between the corner and the center of a side. To absorb the thermal expansion of the test tubes by heating, a flexible tube is connected at the bottom of the test section. The outer walls of the test tubes are thermally well insulated by ceramic-wool which is suitable for the high temperature insulation and is wrapped by ceramic-tape. To measure conduction to the non-heating region of the test tube, two thermocouples are attached upstream of the starting position of the heating region. The fluid temperature is measured at the inlet and outlet mixing chamber of the test section by using the same type of thermocouples.

Table 7-2. Dimensions of test sections.

\begin{tabular}{lcccc}
\hline & Small circular tube & Triangular tube & Square tube & Large circular tube \\
\hline Hydraulic diameter [mm] & 7.8 & 9.8 & 7.9 & 19.4 \\
Thickness [m] & 1.0 & 1.0 & 1.0 & 0.8 \\
Heated length [m] & 1.2 & 1.2 & 1.2 & 1.36 \\
Entrance length [m] & 0.8 & 0.8 & 0.8 & 0.6 \\
\hline
\end{tabular}

\section{Experimental method}

The measured quantities listed in the Table 7-3 are the outer wall temperature, inlet / outlet fluid temperature at the test section and the static pressure by using thermocouples and pressure transmitters, respectively. In order to measure the wall temperature, a FLUKE data acquisition unit is used. And then the data is transferred and stored with a personal computer by using a RS232C cable. The data acquired from the thermocouples are calibrated by the RTD (Resistance Temperature Detector) to within $\pm 0.1 \mathrm{C}$.

The outer wall temperatures are measured for different experimental conditions such as inlet temperature, heat flux, and mass velocity within the ranges as shown in Table 7-1 at the constant system pressure of $8 \mathrm{MPa}$. The Reynolds numbers based on the inlet conditions are in the range $1.9 \times 10^{3}$ to $1.9 \times 10^{5}$ and Grashof numbers vary from $5 \times 10^{9}$ to $4 \times 10^{11}$ (based on the small circular tube diameter).

The fluid enthalpy at any position in the test section is calculated from the measured values of inlet fluid temperature, mass flow rate and heat flux by considering the energy balance of Equation (7-1).

$$
H_{x}=H_{i}+\frac{q^{\prime \prime} A}{\dot{m}},
$$

where $H_{x}$ and $H_{i}$ refer to the enthalpy $(\mathrm{J} / \mathrm{kg})$ of carbon-dioxide in the axial location $x$ and inlet of the test section and $q^{\prime \prime}, A$, and $\dot{m}$ are the heat flux $\left(\mathrm{W} / \mathrm{m}^{2}\right)$, heat transfer area $\left(\mathrm{m}^{2}\right)$, and mass flow rate $(\mathrm{kg} / \mathrm{s})$, respectively. The bulk fluid temperature at the location $x$ can be determined from the calculated fluid enthalpy according to the carbondioxide property tables. Thermodynamic properties are evaluated from the NIST Chemistry Web book and the NIST Refrigerant Properties Database 6.0 [McKinden, et al., 1998] . The uncertainties of the experimental results are explained at the end of this task report. In this report, the Nusselt number, Reynolds number, Prandtl number and Grashof number are calculated based on the bulk fluid and wall temperature as follows:

$$
N u=\frac{h D_{h}}{k}=\frac{q^{\prime \prime} D_{h}}{k\left(T_{w}-T_{b}\right)}
$$




$$
\begin{gathered}
\mathrm{Re}=\frac{\rho u D_{h}}{\mu} \\
\operatorname{Pr}=\frac{\mu c_{p}}{k} \\
G r=\frac{g \beta q^{\prime \prime} D_{h}^{4}}{k v^{2}}
\end{gathered}
$$

The properties in Equations (7-2 to 7-5) are evaluated at the inlet temperature which is measured by a thermocouple at the test sections inlet. (When local bulk properties are used, a subscript "b" is added.)

Table 7-3. Measured variables and instrumentation.

\begin{tabular}{lll}
\hline \multicolumn{1}{c}{ Variable } & \multicolumn{1}{c}{ Device } & \multicolumn{1}{c}{ Remarks } \\
\hline Outer wall temperature $(\mathrm{C})$ & K-type unground thermocouple & using FLUKE \\
Fluid temperature $(\mathrm{C})$ & K-type unground thermocouple & $\pm 0.1 \%$ f.s..of reading \\
Pressure $($ bar) & Pressure transmitter & $\pm 0.5 \%$ f.s. of reading \\
Mass flow rate $(\mathrm{kg} / \mathrm{s})$ & Coriolis type mass flowmeter & \\
\hline
\end{tabular}

Small circular tube experiments Prof. Lee conducted heat transfer experiments of supercritical carbon dioxide flow at $8 \mathrm{MPa}$ on a small vertical circular tube. The pseudocritical temperature $\mathrm{T}_{\mathrm{pc}}$ is about $34.7 \mathrm{C}$. By varying heat flux, mass velocity and inlet bulk temperature, sixty eight experiments were performed. Experimental conditions are listed in Table 7-4. The effects of heat flux, mass velocity (flux) and inlet bulk temperature on heat transfer in a vertical tube were investigated. The operating range of mass velocity is varied from 209 to $1260 \mathrm{~kg} / \mathrm{m}^{2} \mathrm{~s}$ and the inlet temperatures are 15,25 and $32 \mathrm{C}$. The heat flux supplied by the test section varied from 5 to 180 $\mathrm{kW} / \mathrm{m}^{2}$. Under these conditions, the bulk fluid temperature covers the liquid, supercritical and pseudocritical temperature with the wall temperature above pseudocritical temperature. The following experimental results of wall temperature distributions along the streamwise direction are expressed with the dimensionless heating length $x / D_{h}$ at the same inlet temperature and mass velocity with increasing heat flux. For the case of minimum mass velocity of $209 \mathrm{~kg} / \mathrm{m}^{2} \mathrm{~s}$, the results are explained later because the results differ from that of the those test conditions.

Figure 7-4 shows the wall temperature distributions for a mass velocity of $314 \mathrm{~kg} / \mathrm{m}^{2} \mathrm{~s}$ at an inlet bulk temperature of $15 \mathrm{C}$ with increasing heat flux from 15 to $40 \mathrm{~kW} / \mathrm{m}^{2}$. At the lower heat fluxes of 15 and $20 \mathrm{~kW} / \mathrm{m}^{2}$, the wall temperatures exhibit almost linear increases along the heating region. However, at the heat flux of 23 $\mathrm{kW} / \mathrm{m}^{2}$, double peak of wall temperature appear. Further increases in heat flux cause the first peak to develop and move toward the upstream region. 
Table 7-4: Experimental conditions for the small circular tube

\begin{tabular}{ccccc}
\hline $\begin{array}{c}\text { Mass flow rate } \\
(\mathrm{kg} / \mathrm{s})\end{array}$ & $\begin{array}{c}\text { Mass velocity } \\
\left(\mathrm{kg} / \mathrm{m}^{2} \mathrm{~s}\right)\end{array}$ & $\begin{array}{c}\text { Inlet temperature } \\
\left({ }^{\circ} \mathrm{C}\right)\end{array}$ & $\begin{array}{c}\text { Heat flux } \\
\left(\mathrm{kW} / \mathrm{m}^{2}\right)\end{array}$ & $\operatorname{Re}$ \\
\hline 0.01 & 209 & 15 & $5,10,15,30,40,50,60,70$ & 19,000 \\
0.015 & 314 & 15 & $5,20,23,25,30,35,40$ & 29,000 \\
& & 15 & $20,30,35,38,39,40,50,60$ & 39,000 \\
0.02 & 419 & 25 & $20,30,40,60$ & 49,000 \\
& & 32 & $20,30,40,50,60$ & 65,000 \\
0.025 & 523 & 15 & $50,60,70,80$ & 49,000 \\
0.03 & 628 & 15 & $50,60,65,70,80,85,90,100,110$ & 58,000 \\
& & 20 & $20,60,100,120,140,160,180$ & 65,000 \\
0.04 & \multirow{2}{*}{337} & 15 & $20,60,100$ & 98,000 \\
& & 25 & $20,60,100$ & 130,000 \\
0.06 & 1260 & 15 & $20,60,100$ & 120,000 \\
& & 25 & $20,60,100$ & 147,000 \\
& & 32 & $20,60,100$ & 196,000 \\
\hline
\end{tabular}

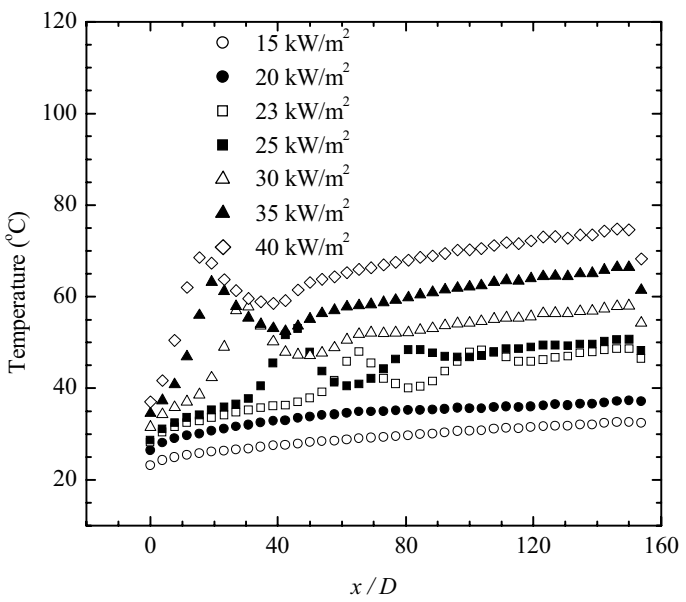

Fig. 7-4. Wall temperature distributions for the small circular tube with increasing heat flux at a mass velocity of $314 \mathrm{~kg} / \mathrm{m}^{2} \mathrm{~s}$.

For the mass velocity of $419 \mathrm{~kg} / \mathrm{m}^{2} \mathrm{~s}$, experiments are conducted for the three different inlet bulk temperature conditions as shown in Figures 7-5. Figure 7-5(a) represents the occurrence of deterioration in heat transfer, when the inlet temperature is $15^{\circ} \mathrm{C}$. In comparison with the lower mass velocity (Figure 7-4), the wall temperature peak appears at a relatively higher heat flux of $35 \mathrm{~kW} / \mathrm{m}^{2}$. When the bulk inlet temperature increases higher, heat transfer "deterioration" hardly occurs as shown in Figure 7-5(b, c). In contrast to the normal heat transfer at an inlet temperature of $32 \mathrm{C}$, the wall temperature for a bulk inlet temperature of $25 \mathrm{C}$ shows deterioration in heat transfer at the heat flux of $40 \mathrm{~kW} / \mathrm{m}^{2}$. It is believed that buoyancy is less for $T_{\text {in }}=366$ due to the temperature difference between the near-wall pseudocritical temperature and the bulk fluid temperature being decreased. 


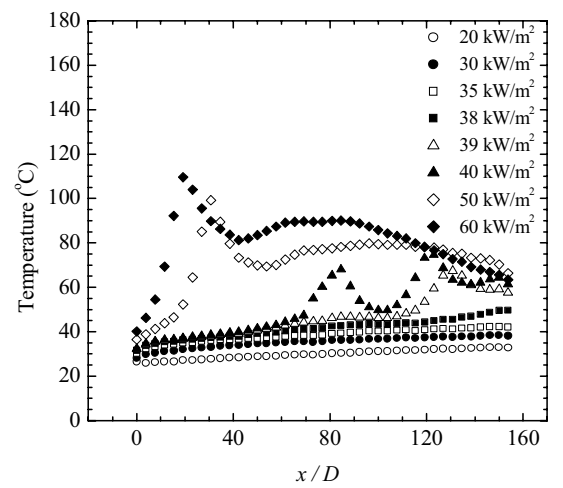

(a) Inlet temperature $15^{\circ} \mathrm{C}$

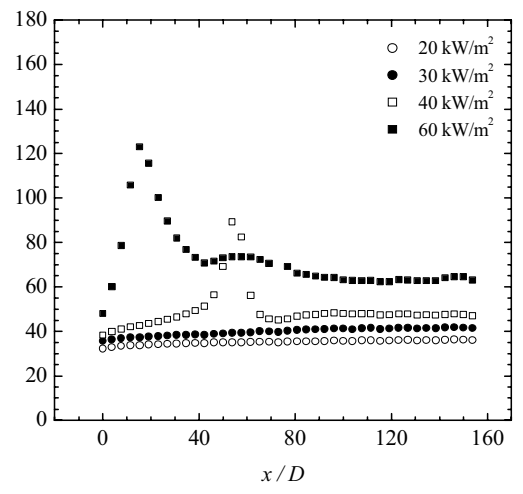

(b) Inlet temperature $25{ }^{\circ} \mathrm{C}$

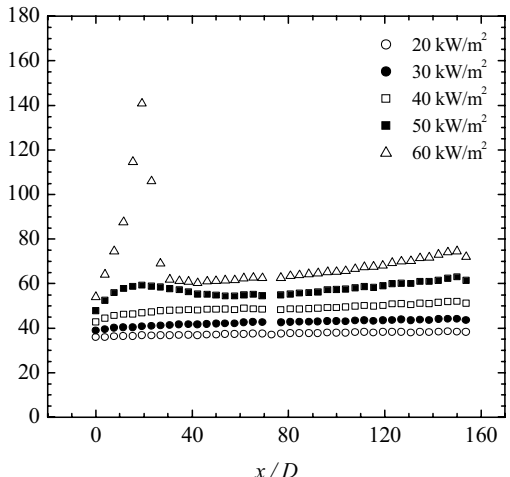

(c) Iinlet temperature $32{ }^{\circ} \mathrm{C}$

Fig. 7-5. Wall temperature distributions of a small circular tube with increasing heat fluxes at the mass velocity of $419 \mathrm{~kg} / \mathrm{m}^{2} \mathrm{~s}$.

The remaining experimental results for higher mass velocities of $523 \mathrm{~kg} / \mathrm{m}^{2} \mathrm{~s}$ (Figure 7-6(a)) and $628 \mathrm{~kg} / \mathrm{m}^{2} \mathrm{~s}$ (Figure 7-6(b)) exhibit tends which are comparable to those observed for lower mass velocities. The peak wall temperature occurs at higher heat flux and lower mass velocity and then moves upstream with further increase of heat flux.

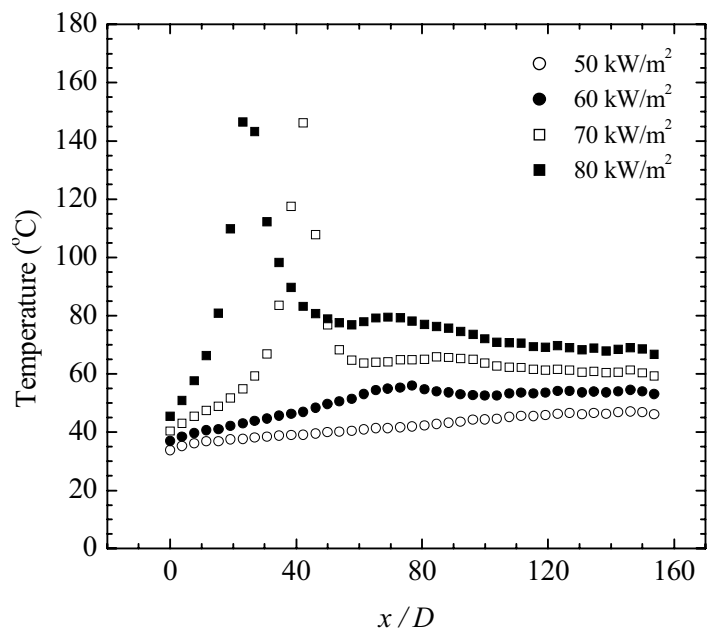

(a) $523 \mathrm{~kg} / \mathrm{m}^{2} \mathrm{~s}$

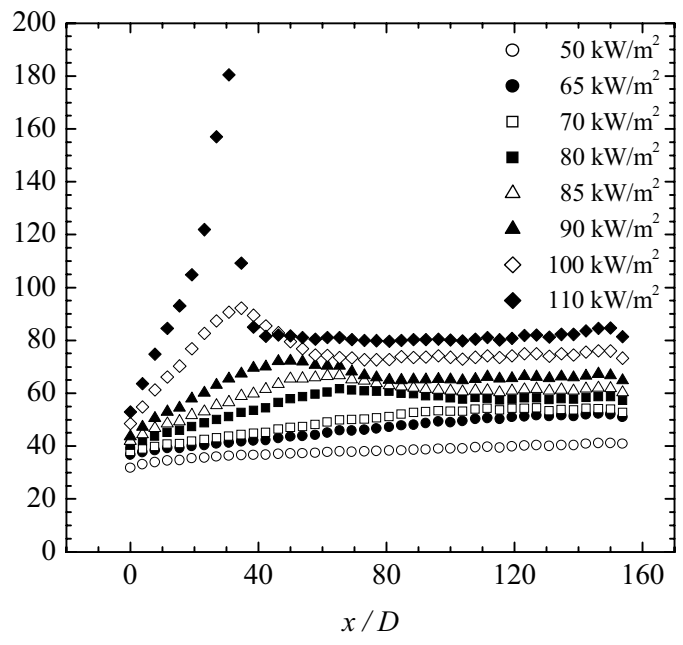

(b) $628 \mathrm{~kg} / \mathrm{m}^{2} \mathrm{~s}$

Fig. 7-6. Wall temperature distributions for the small circular tube with increasing heat flux at mass velocities of (a) $523 \mathrm{~kg} / \mathrm{m}^{2} \mathrm{~s}$ and (b) $628 \mathrm{~kg} / \mathrm{m}^{2} \mathrm{~s}$.

The wall temperature behavior at a mass velocity of $209 \mathrm{~kg} / \mathrm{m}^{2} \mathrm{~s}$ is plotted in Figure 7-7. As can be seen in the figure, the two cases of 60 and $70 \mathrm{~kW} / \mathrm{m} 2$ show different wall temperature distributions from the lower heat flux conditions at the same mass velocity. The wall temperature increases from the inlet until it reaches a local peak and then decreases to a minimum where the bulk fluid temperature becomes closer to the pseudocritical temperature as shown in Figure 7-8. When the bulk fluid temperature exceeds the pseudocritical temperature, the wall temperatures increase again. Similar behavior was also observed in the previous works of Wood and Smith [1964] and Yamagata et al. [1972]. This effect is due mainly to the rapid variations of the fluid properties near the pseudocritical temperature. As the bulk fluid temperature approaches the pseudocritical temperature, the thermal conductivity decreases, but at the same time the specific heat has the maximum value and the viscosity decreases. These variations imply that the turbulence is less damped by reduction in viscosity and the effective heat diffusion is guaranteed by high value of specific heat. As the bulk temperature increases beyond the pseudocritical temperature, 
the wall temperature increases again due to the sharp reduction in the specific heat and the fluid begins to behave more like a normal gas.

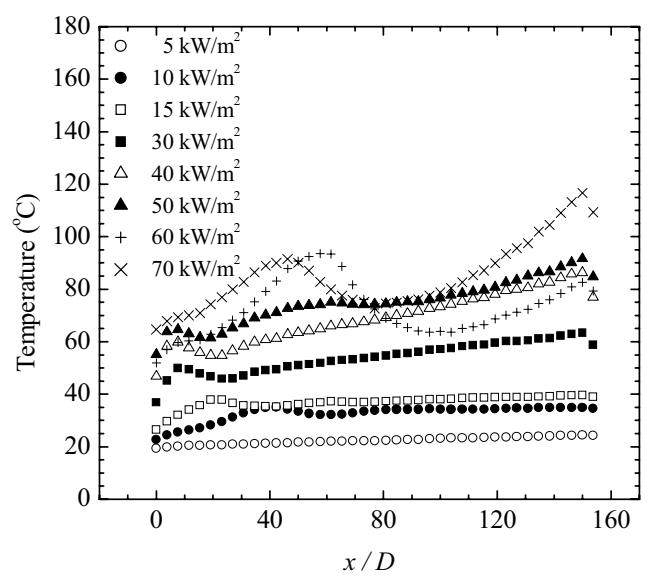

Fig. 7-7. Wall temperature distributions for the small circular tube with increasing heat flux at are mass velocity of $209 \mathrm{~kg} / \mathrm{m} 2 \mathrm{~s}$.

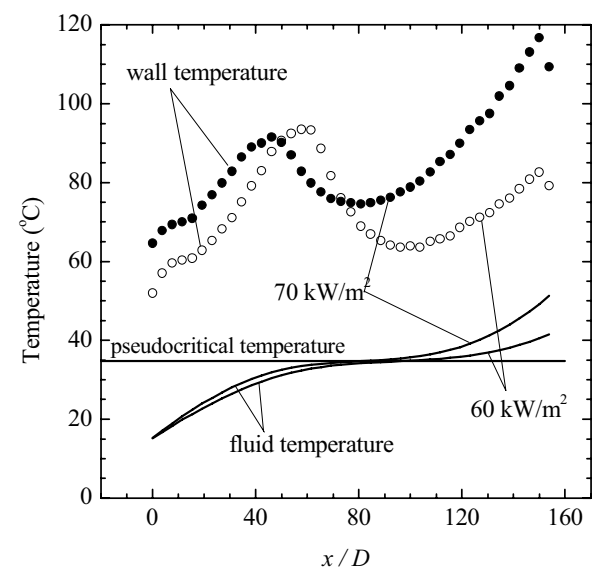

Fig. 7-8. Wall temperature distributions for the small circular tube for two different heat fluxes at a mass velocity of $209 \mathrm{~kg} / \mathrm{m} 2 \mathrm{~s}$.

\section{Non-circular tube experiments}

Until now, heat transfer experiments in non-circular tubes were conducted mainly for gas flow. Campbell and Perkins [1970] and Battista and Perkins [1970] measured the effects of variable properties on local heat transfer coefficients of fully-developed turbulent air flow in vertical noncircular tubes. Campbell and Perkins [1970] found that the local heat transfer coefficients in an equilateral triangular duct are lower than those in a circular duct by about ten per cent. In an investigation of heat transfer in a square duct, Battista and Perkins [1970] observed that the local heat transfer coefficient is about the same as that in the equilateral triangular duct.

To the knowledge of the author, the present study is the first on heat transfer of supercritical flow in noncircular tubes. In order to compare the effect of cross-section shape on supercritical heat transfer, experiments were performed at the same experimental conditions of inlet bulk temperature, mass velocity and heat flux applied to the small circular tube as listed in Tables 7-5 and 7-6. However, the total heat input and mass flow rates are different each other because flow passage and heating areas are different from one to another. The cross-section shape of the non-circular tubes are equilateral triangular and square so the apex angle with rounded corner is wide enough to use the hydraulic diameter to calculate the dimensionless parameters and heat transfer coefficients of the supercritical flow in non-circular tubes [Carlson and Irvine, 1961; Hartnett, Koh and McComas, 1962]. To examine the uniformity of the wall temperature peripherally, measurements are performed at the center of a side, a corner and a mid-point between the center and the corner (a quarter of a side from the corner) at each axial location in the heating region. The following results for the non-circular tubes are expressed in terms of the wall temperature at the center. As with the small circular tube, experimental data are obtained by increasing heat flux at each mass velocity.

The results for the non-circular tubes show that the wall temperature distributions along the streamwise direction have similar trends to those of the circular tube at the same heat flux and mass velocity. For the mass velocity of $314 \mathrm{~kg} / \mathrm{m}^{2} \mathrm{~s}$, double peaks in the wall temperature appeared at a heat flux of $23 \mathrm{~kW} / \mathrm{m}^{2}$ as shown in Figure 7-9. With further increase in the heat flux, a strong peak wall temperature moves upstream as noted for the circular tube at the same experimental conditions. However, the location of the initial peak wall temperature is upstream from that for the circular tube. For example, at $q^{\prime \prime}=23 \mathrm{~kW} / \mathrm{m}^{2}$, the temperature peak appears at $x / D_{h}=39$, 47 and 65 for the triangular, square and circular tubes, respectively, whereas it occurs at about $x / D_{h}=15$ for all tubes when the heat flux is $40 \mathrm{~kW} / \mathrm{m}^{2}$. 
Table 7-5. Experimental conditions for the triangular tube.

\begin{tabular}{ccccc}
\hline $\begin{array}{c}\text { Mass flow rate } \\
(\mathrm{kg} / \mathrm{s})\end{array}$ & $\begin{array}{c}\text { Mass velocity } \\
\left(\mathrm{kg} / \mathrm{m}^{2} \mathrm{~s}\right)\end{array}$ & $\begin{array}{c}\text { Inlet temperature } \\
(\mathrm{C})\end{array}$ & $\begin{array}{c}\text { Heat flux } \\
\left(\mathrm{kW} / \mathrm{m}^{2}\right)\end{array}$ & $\operatorname{Re}_{\mathrm{Dh}, \text { in }}$ \\
\hline 0.032 & 314 & 15 & $15,20,23,25,30,35,40$ & 36,000 \\
& & 15 & $20,30,35,38,39,40,50,60$ & 48,000 \\
0.043 & 419 & 25 & $20,30,40,60$ & 61,000 \\
& & 32 & $20,30,40,50,60$ & 81,000 \\
0.054 & 523 & 15 & $50,60,70,80$ & 60,000 \\
0.065 & 628 & 15 & $50,65,70,80,85,90,100,110$ & 72,000 \\
& & 15 & $20,60,100,120.140$ & 96,000 \\
0.086 & 837 & 25 & $20,60,100$ & 121,000 \\
& & 32 & 20 & 162,000 \\
\hline
\end{tabular}

Table 7-6. Experimental conditions of the square tube.

\begin{tabular}{ccccc}
\hline $\begin{array}{c}\text { Mass flow rate } \\
(\mathrm{kg} / \mathrm{s})\end{array}$ & $\begin{array}{c}\text { Mass velocity } \\
\left(\mathrm{kg} / \mathrm{m}^{2} \mathrm{~s}\right)\end{array}$ & $\begin{array}{c}\text { Inlet temperature } \\
(\mathrm{C})\end{array}$ & $\begin{array}{c}\text { Heat flux } \\
\left(\mathrm{kW} / \mathrm{m}^{2}\right)\end{array}$ & $\operatorname{Re}_{\mathrm{Dh}, \text { in }}$ \\
\hline 0.0199 & 313 & 15 & $20,23,30,40$ & 30,000 \\
& & 15 & $35,38,40,50,60$ & 40,000 \\
0.0266 & 419 & 25 & $30,40,60$ & 50,000 \\
& & 32 & $30,40,50,60$ & 67,000 \\
0.0332 & 523 & 15 & $50,60,70$ & 50,000 \\
0.0399 & 628 & 15 & $70,80,85,90,100,110$ & 60,000 \\
\hline
\end{tabular}

The occurrence of an earlier wall temperature peak at a lower inlet temperature for the circular tube (Figure 7-5) are also observed in the non-circular tubes as shown in Figure 7-10 which shows results for three different bulk inlet temperatures of 15,25 and $32 \mathrm{C}$ at $419 \mathrm{~kg} / \mathrm{m}^{2} \mathrm{~s}$. With increasing inlet temperature, heat transfer deterioration hardly occurs, however, for $T_{i n}=32 \mathrm{C}, q^{\prime \prime}=60 \mathrm{~kW} / \mathrm{m}^{2}$, the peak wall temperature is higher than those for the lower inlet temperatures at the same heat flux. For example, the peak wall temperature is about $140 \mathrm{C}$ at $T_{i n}=32 \mathrm{C}, q^{\prime \prime}=$ $60 \mathrm{~kW} / \mathrm{m}^{2}$; on the contrary, the peak wall temperatures are about $100 \mathrm{C}$ and $120 \mathrm{C}$ for inlet temperatures of 15 and $25 \mathrm{C}$, respectively. Therefore, the sharp increase and decrease in the wall temperature distributions at the inlet temperature of $32 \mathrm{C}$ as shown in Figures 7-10(c, f), are results of a delay of heat transfer deterioration and the higher wall temperature peaks at the same heat flux.

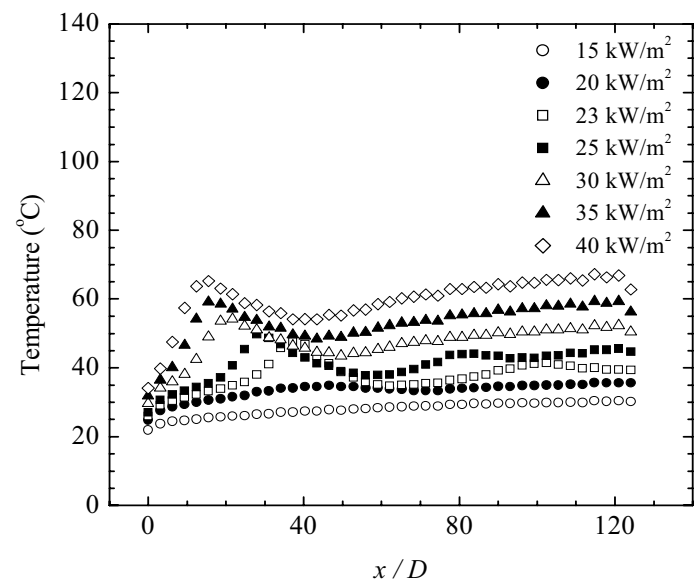

(a) Triangular tube

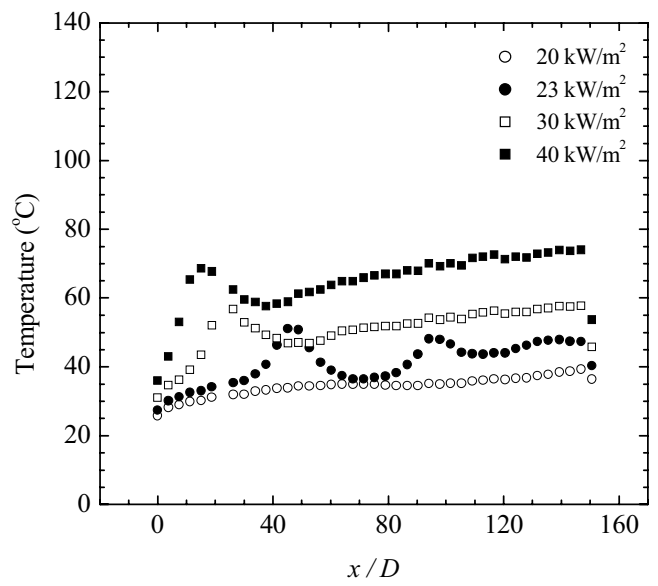

(b) Square tube

Fig. 7-9. Wall temperature distributions for non-circular tubes at a mass velocity of $314 \mathrm{~kg} / \mathrm{m}^{2} \mathrm{~s}$. 


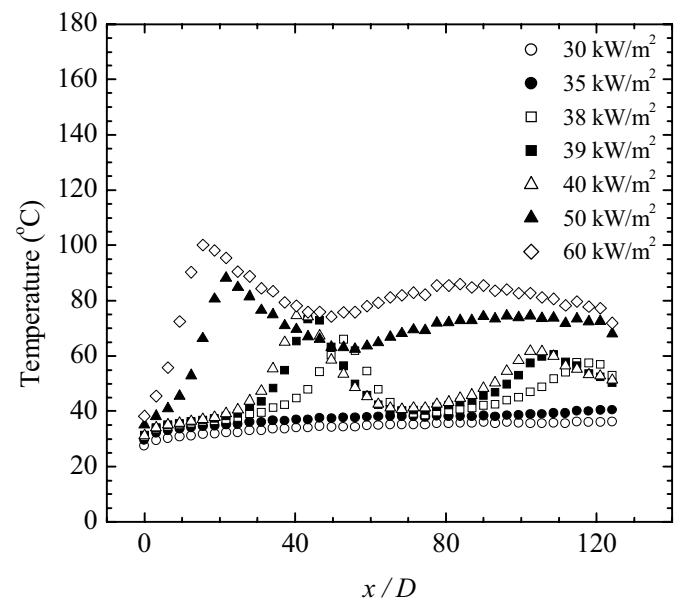

(a) Inlet temperature $15^{\circ} \mathrm{C}$, triangular tube

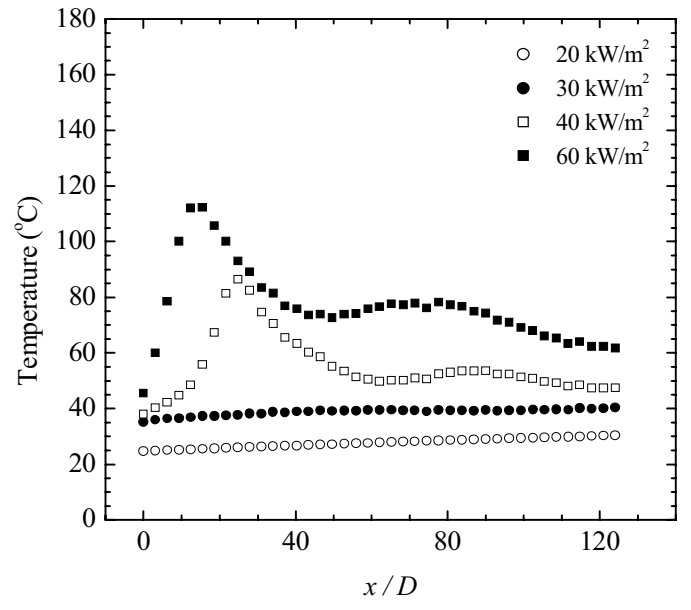

(b) Iinlet temperature $25^{\circ} \mathrm{C}$, triangular tube

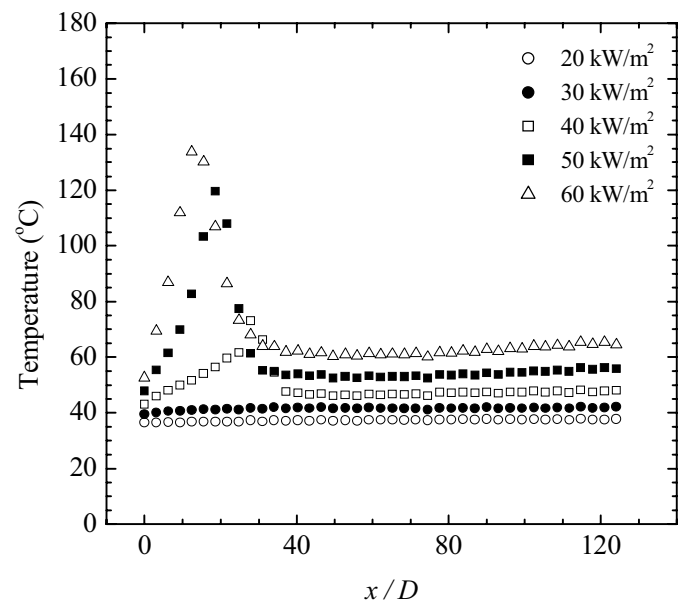

(c) Inlet temperature $32{ }^{\circ} \mathrm{C}$, triangular tube

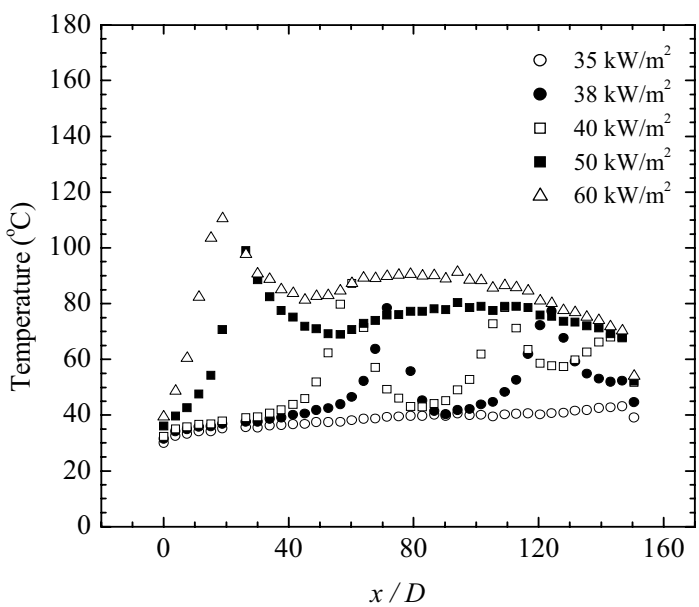

(d) Inlet temperature $15^{\circ} \mathrm{C}$, square tube

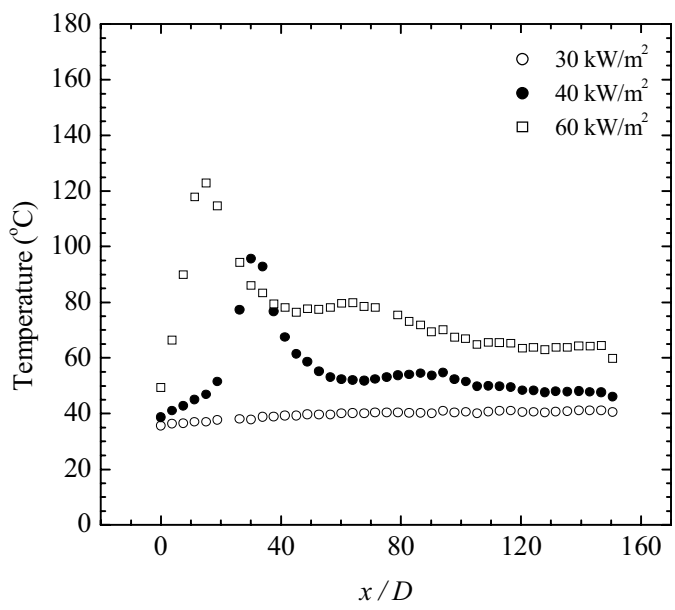

(e) Inlet temperature $25^{\circ} \mathrm{C}$, square tube

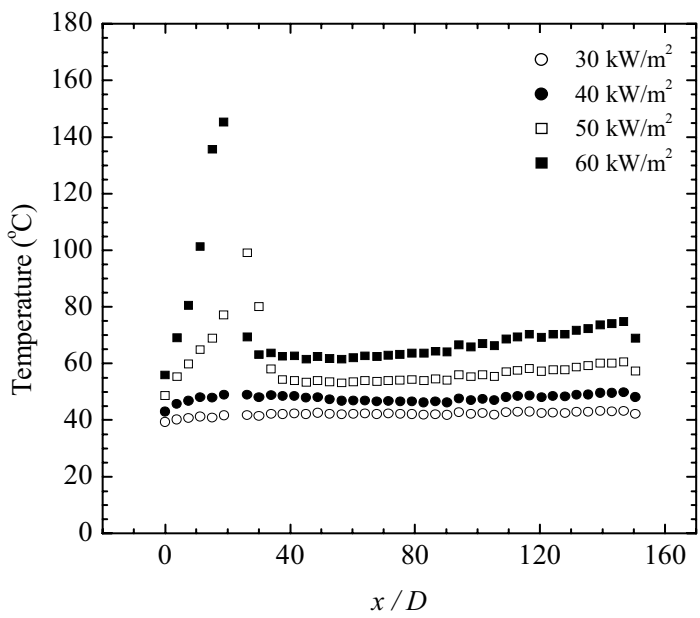

(f) Inlet temperature $32{ }^{\circ} \mathrm{C}$, square tube

Fig. 7-10. Wall temperature distributions for the non-circular tubes at a mass velocity of $419 \mathrm{~kg} / \mathrm{m}^{2} \mathrm{~s}$. 
The results for the mass velocity of 523 and $628 \mathrm{~kg} / \mathrm{m}^{2} \mathrm{~s}$ are shown in Figures 7-11 and 7-12. It is also apparent from the foregoing comparisons that the wall temperature distributions for the non-circular tubes are almost the same as for the circular tube as a whole; however, an earlier heat transfer deterioration is evident in the noncircular tubes. For a given heat flux,, more power is supplied to the non-circular tubes than to the circular tube because the heating areas of non-circular tubes are larger than that of the circular tube.

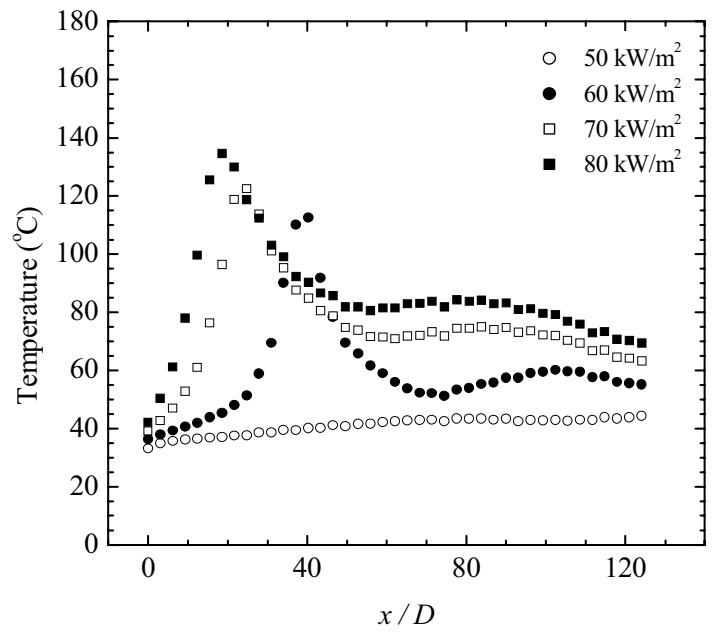

(a) Triangular tube

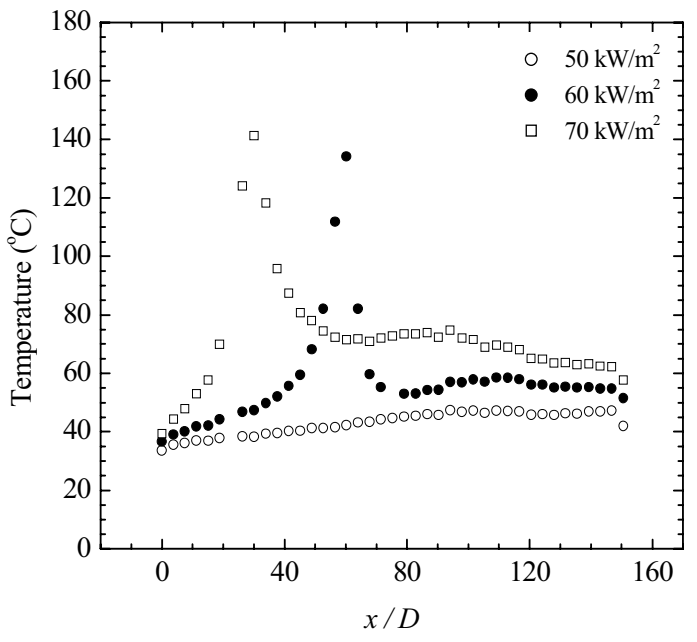

(b) Square tube

Fig. 7-11. Wall temperature distributions for non-circular tubes at a mass velocity of $523 \mathrm{~kg} / \mathrm{m}^{2} \mathrm{~s}$.

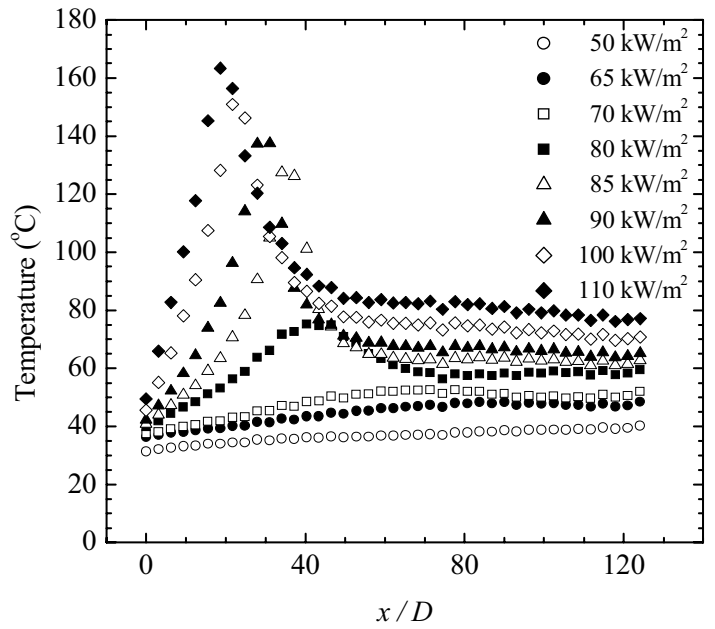

(a) Triangular tube

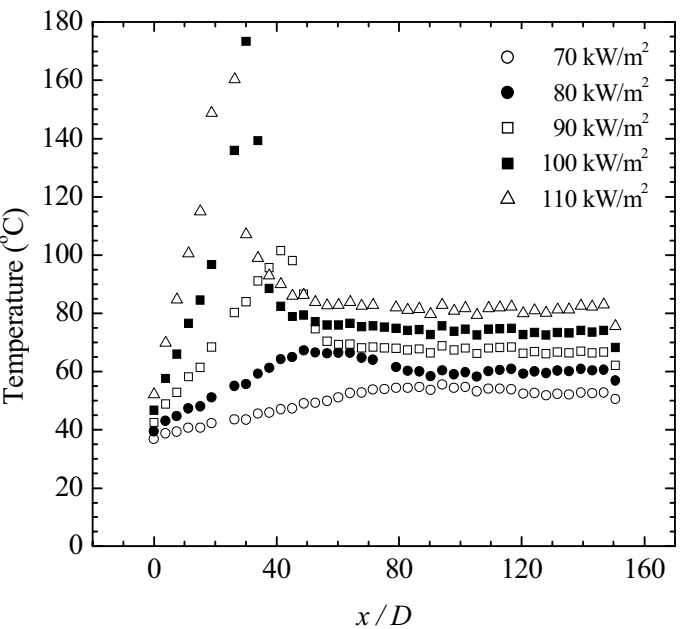

(b) Square tube

Fig. 7-12. Wall temperature distributions for non-circular tubes along the axial direction at a mass velocity of 628 $\mathrm{kg} / \mathrm{m}^{2} \mathrm{~s}$.

Figure 7-13 shows the peripheral uniformity of wall temperature at each location. The temperature at the corner is slightly higher than at other positions. On the average, for all experimental conditions with the triangular tube, the temperatures at the corner and quarter are slightly higher than at the center by about $5.5 \mathrm{C}$ and $0.8 \mathrm{C}$, which are about 10.4 per cent and 1.5 per cent higher, respectively. For the square tube, similarly, the temperature differences between the corner, quarter and the center are about $5.2 \mathrm{C}$ and $2.8 \mathrm{C}$, i.e. 4.5 per cent and 2.4 per cent higher at the corner and quarter locations, respectively. The reason is believed to be that the convective heat transfer 
at the corner is reduced because the flow velocity near the corner is slower than the sides. With increasing heat flux, the temperature difference between the corner and the side becomes larger.

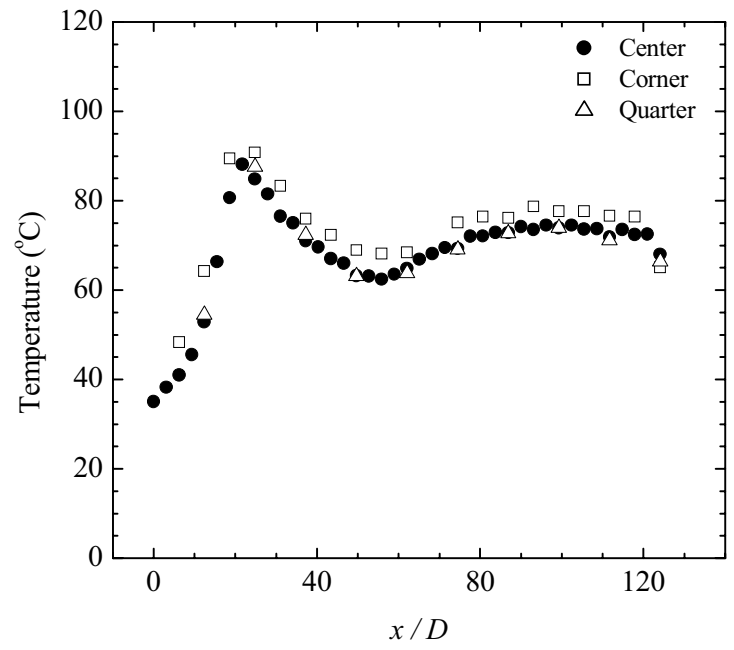

(a) triangular tube

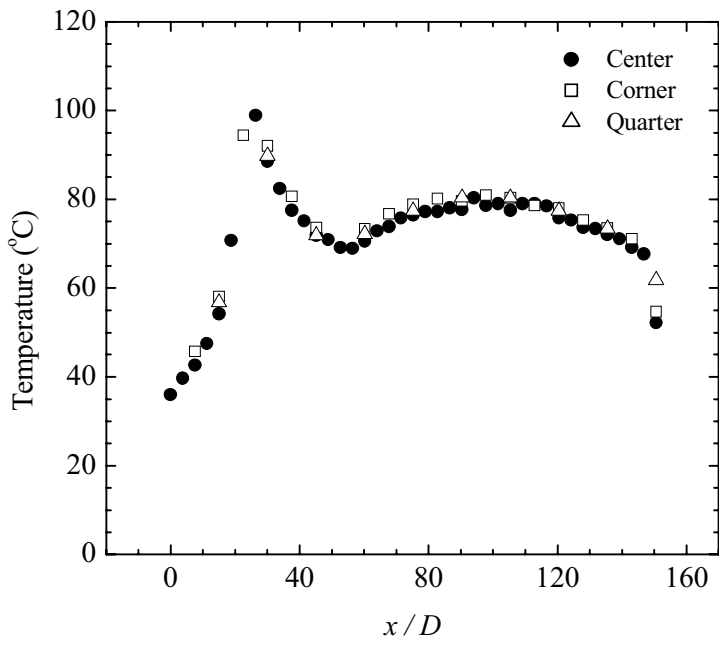

(b) square tube

Fig. 7-13. Wall temperature distributions at the center, corner and quarter positions of non-circular tubes at a mass velocity of $419 \mathrm{~kg} / \mathrm{m}^{2} \mathrm{~s}$.

Large circular tube experiments As for the non-circular tube experiments, some conditions of the mass velocity and heat flux for the small circular tube are applied to the large circular tube at the inlet temperature of 15 C. The experimental conditions are listed in Table 7-7. The heating region of seventy diameters for the large circular tube is shorter than the 150 diameters for the small circular tube. Therefore, the effect of heating length on heat transfer is investigated. Celata et al. [1998] observed that the heat transfer decreases as increasing the heating length in aiding mixed convective flow. However, from the results of the Celata et al. [1998], the heating length of seventy diameters in this experiment is long enough to neglect the length effect on heat transfer downstream.

For a mass velocity of $126 \mathrm{~kg} / \mathrm{m}^{2} \mathrm{~s}$, Figure $7-14$ shows that the wall temperature increasing in the upstream region for $5 \mathrm{~kW} / \mathrm{m}^{2}$ but the peak wall temperature appears to be at the first measurement position in the heated region for heat fluxes of 50 and $70 \mathrm{~kW} / \mathrm{m}^{2}$. Figure $7-8$ for the small circular tube, the same is observed at the heat flux of $70 \mathrm{~kW} / \mathrm{m}^{2}$ in Figure 7-15. After the fluid temperature passes through the pseudocritical temperature, the wall temperature decreases and then rises. 
Table 7-7. Experimental conditions for the large circular tube.

\begin{tabular}{ccccc}
\hline $\begin{array}{c}\text { Mass flow rate } \\
(\mathrm{kg} / \mathrm{s})\end{array}$ & $\begin{array}{c}\text { Mass velocity } \\
\left(\mathrm{kg} / \mathrm{m}^{2} \mathrm{~s}\right)\end{array}$ & $\begin{array}{c}\text { Inlet temperature } \\
(\mathrm{C})\end{array}$ & $\begin{array}{c}\text { Heat flux } \\
\left(\mathrm{kW} / \mathrm{m}^{2}\right)\end{array}$ & $\operatorname{Re}$ \\
\hline 0.0373 & 126 & 15 & $1,5,15,20,30,50,70$ & 30,000 \\
0.0619 & 209 & 15 & $5,10,20,30,40,50,60,70$ & 48,000 \\
0.0928 & 314 & 15 & $15,20,25,30,40,50,60,70$ & 72,000 \\
0.1237 & 419 & 15 & $20,30,35,40,50,60,70$ & 97,000 \\
\hline
\end{tabular}

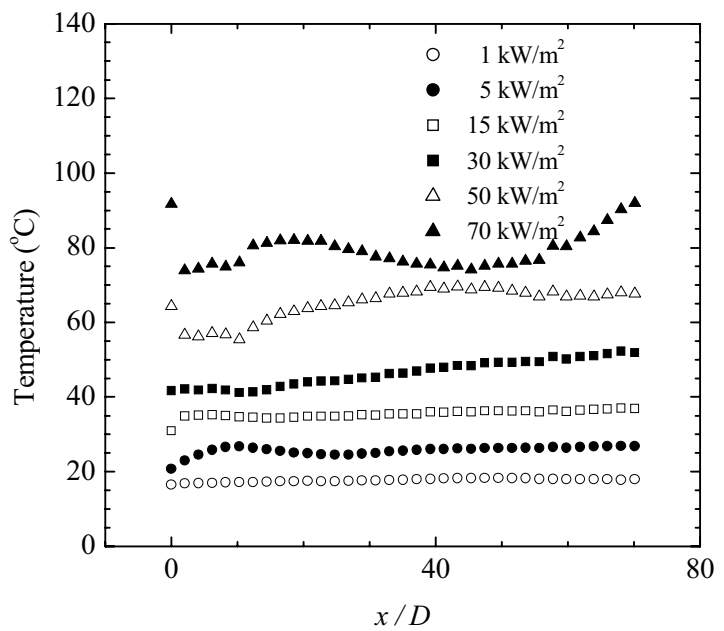

Fig. 7-14. Wall temperature distributions of the large circular tube at a mass velocity of $126 \mathrm{~kg} / \mathrm{m}^{2} \mathrm{~s}$.

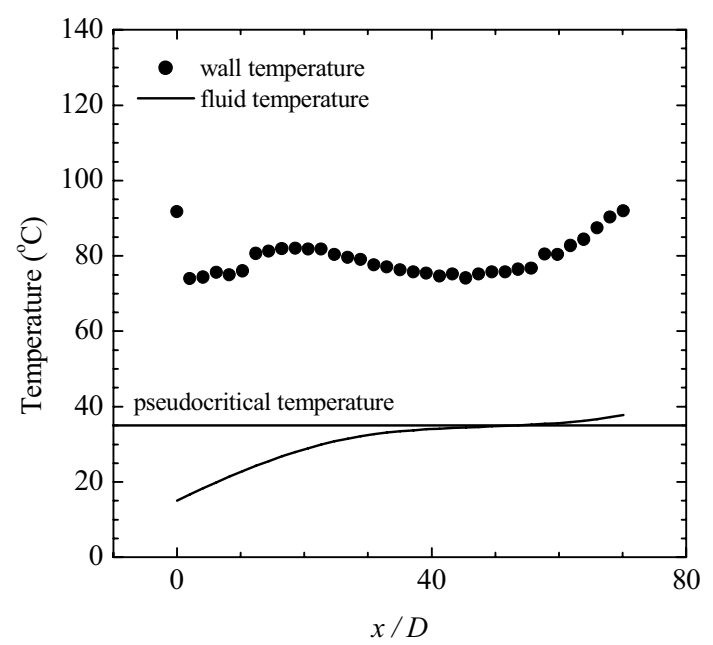

Fig. 7-15. Wall and bulk temperature distributions for the large circular tube at a mass velocity of $126 \mathrm{~kg} / \mathrm{m}^{2} \mathrm{~s}$ and heat flux of $70 \mathrm{~kW} / \mathrm{m}^{2}$.

For the higher mass velocities of 209,314 and $419 \mathrm{~kg} / \mathrm{m}^{2} \mathrm{~s}$, the wall temperature distributions are shown in Figure 7-16. The temperature distributions for each heat flux are similar with that of the small circular tube and the beginnings of the deterioration in heat transfer are almost the same as the results for the small circular tube. These observations do not agree with previous studies of the effect of tube diameter on the heat transfer. Ackerman [1970] conducted a heat transfer experiment for supercritical pressure water using tubes with inner diameters from 9.4 to 24 $\mathrm{nn}(0.37$ to $0.93 \mathrm{inch})$ and he observed that the use of smaller tubes allows higher heat fluxes before initiation of heat transfer deterioration for given pressure and mass velocity. Shiralkar and Griffith [1970] observed that under equivalent conditions of mass velocity and heat flux in supercritical carbon-dioxide flow, the smaller test tube diameter has the effect of reducing the degree of deterioration in heat transfer. According to the present results, the heat transfer deterioration due to the buoyancy is about the same for the small and large circular tubes. Thus, mass velocity, heat flux and inlet temperature have dominant effects on the heat transfer at supercritical pressure. 


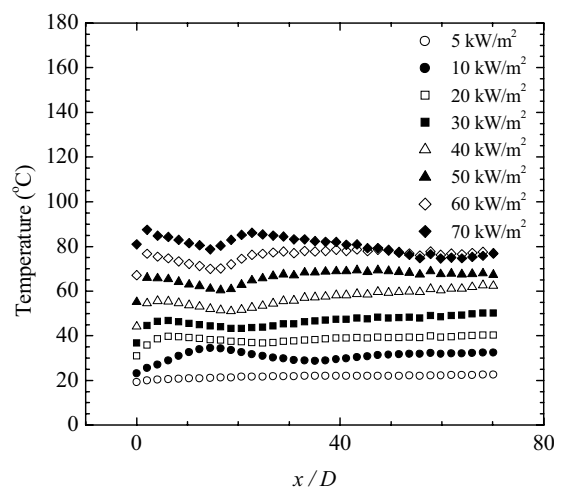

(a) $206 \mathrm{~kg} / \mathrm{m}^{2} \mathrm{~s}$

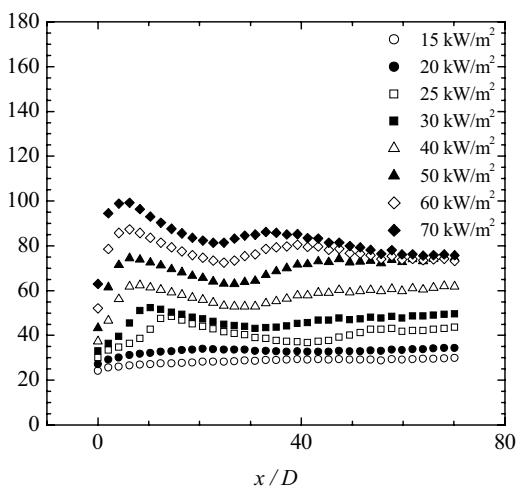

(b) $314 \mathrm{~kg} / \mathrm{m}^{2} \mathrm{~s}$

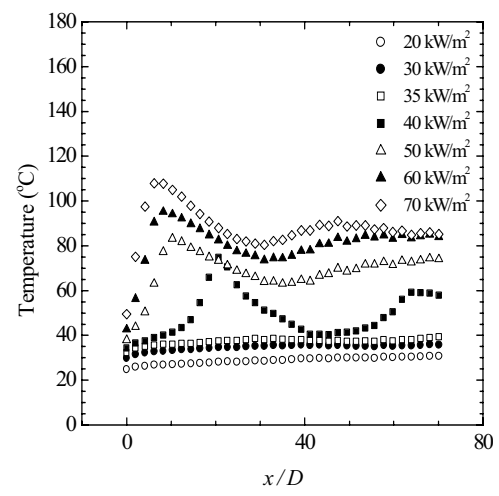

(c) $419 \mathrm{~kg} / \mathrm{m}^{2} \mathrm{~s}$

Fig. 7-16. Wall temperature distributions for the large circular tube at amass velocities: (a) $206 \mathrm{~kg} / \mathrm{m}^{2} \mathrm{~s}$, (b) 314 $\mathrm{kg} / \mathrm{m}^{2} \mathrm{~s}$ and (c) $419 \mathrm{~kg} / \mathrm{m}^{2} \mathrm{~s}$.

Heat transfer criterion

From the experimental results of the small circular tube, we observed that the occurrence of the wall temperature peak depends on both heat flux and mass velocity at the same inlet temperature. It takes place at larger heat fluxes when the mass velocity is high and vice versa, as discussed before. Thus, we can derive a heat transfer deterioration criterion by constructing a regime map from the wall temperature data obtained at all experiment conditions. Figure 7-17 shows the deterioration criterion for the small circular tube as a function of mass velocity and heat flux when the inlet temperature is $15 \mathrm{C}$. If a linear relation is assumed, the best fit can be expressed as

$$
q=0.2 G^{2.0}
$$

where $q$ denotes the heat flux and $G$ is the mass velocity (flux). Similarly, the regime maps for the non-circular and large circular tubes are also drawn in Figures 7-18. In spite of the earlier peak in the wall temperature for the non-circular and large circular tubes, it is found that the same criterion appears to be applicable for a given inlet temperature regardless of the tube cross-sectional shapes and diameter. When the inlet temperatures increase to 25 and $32 \mathrm{C}$, higher heat fluxes are required to induce the deterioration in heat transfer at the same mass velocity compared to the lower inlet temperature case.

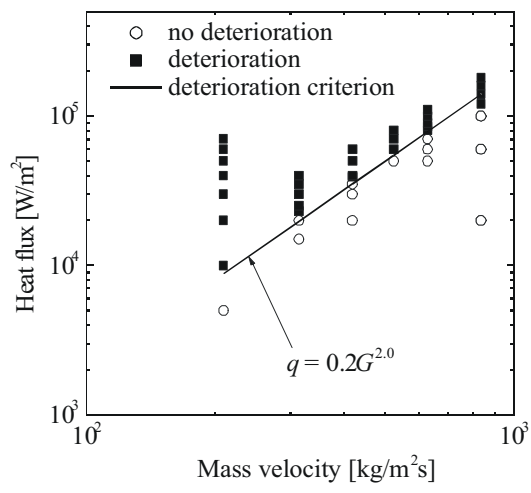

Fig. 7-17. Regime map of the heat transfer deterioration criterion for the small circular tube, inlet temperature $=15$ C.

Summary of heat transfer experiments Experiments with non-circular and large circular tubes were performed at the same conditions of mass velocity and heat flux as imposed on the small circular tube. The 
hydraulic diameters of the small circular, triangular, square, and large circular tubes are 7.8, 9.8, 7.9 and $19.4 \mathrm{~mm}$, respectively. Therefore, the inlet Re numbers and total power supplies to the test section were different from each other at the same conditions of mass velocity and heat flux. Experimental results show that the wall temperature distributions along the streamwise direction are almost the same at the same mass velocity and heat flux with regardless of the hydraulic diameter and cross section shape of the test tubes. When the test conditions are in the range where heat transfer deterioration occurs, the temperature distributions were somewhat different for the four different test tubes. However, the deterioration criterion for the small circular test tube is almost the same as for the non-circular and large circular tubes. The criterion curve is obtained as a function of mass velocity and heat flux. For the non-circular tubes, circumferential uniformity of the wall temperature at a cross section was confirmed. The temperature at the corner is the highest and the temperature at the quarter location of a side is slightly higher than at the center. This slight variation is because the velocity near the corner is lower than at the center.

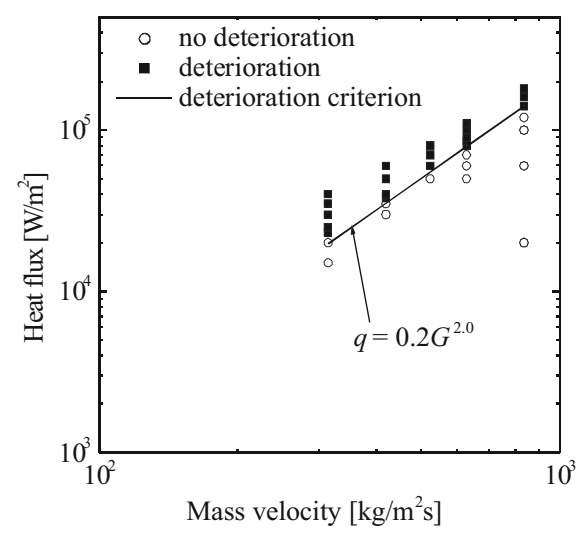

(a) triangular tube

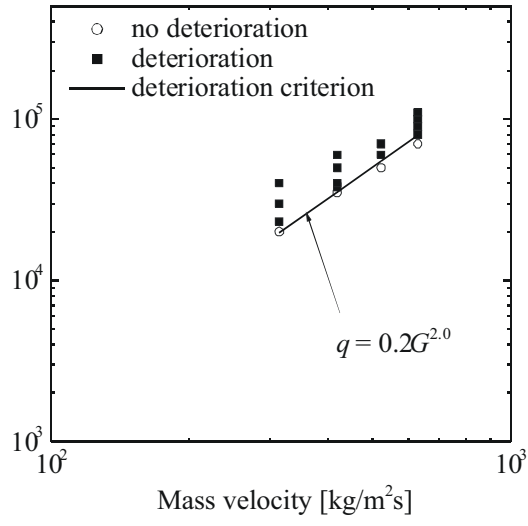

(b) square tube

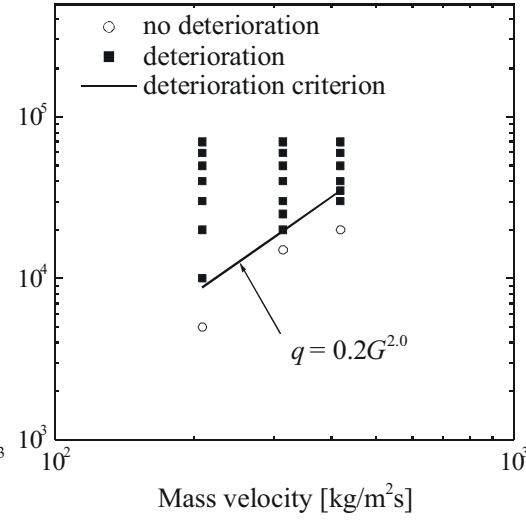

(c) large circular tube

Fig. 7-18. Regime map of the heat transfer deterioration criterion: (a) triangular tube, (b) square tube and (c) large circular tube, inlet temperature $=15 \mathrm{C}$.

New heat transfer correlation Heat transfer at supercritical pressure is strongly affected by the property variation near the pseudocritical temperature and by buoyancy effects. When the fluid temperature in the near-wall region exceeds the pseudocritical temperature, the near-wall fluid density decreases sharply, causing local flow acceleration. Since heat transfer depends on the energy transport from the wall layer to the core of the turbulent flow, change in the velocity distribution due to a buoyancy force and flow acceleration in the near-wall region plays a dominant role at the supercritical pressure.

The velocity gradient between the wall and the core region is smoothed due to flow acceleration and/or buoyancy in the near-wall region. Hence, flow turbulence is suppressed due to the reduction in the shear stress, resulting in an increase in the convective thermal resistance. Thus, the friction coefficient should be included in the formulation of the heat transfer coefficient because the friction coefficient is proportional to the wall shear stress. The friction coefficient $\xi_{M}$ for mixed convection is defined as

$$
\xi_{M}=\frac{8 \tau_{w}}{\rho_{b} u_{b}^{2}},
$$

where $\rho_{\mathrm{b}}$ and $u_{\mathrm{b}}$ are the bulk fluid density and velocity, respectively, and the wall shear stress $\tau_{w}$ is expressed as

$$
\tau_{w}=\rho_{w} u_{\tau}^{2}
$$


where $\rho_{w}$ is the fluid density at the wall temperature, and $u_{\tau}$ is the friction velocity. To calculate $\xi_{M}$, the friction velocity is estimated by evaluating $u_{\tau}$ f at $\mathrm{y}+\approx 30$ in the logrithmic "law",

$$
\frac{u_{b}}{u_{\tau}}=\frac{1}{0.41} \ln \left(\frac{y u_{\tau}}{v}\right)+5.0
$$

The bulk velocity $u_{\mathrm{b}}$ is known..

The friction coefficient $\xi_{F}$ for the forced convection can be calculated by

$$
\xi_{F}=\left[1.8 \log \left(\mathrm{Re}_{b}\right)-1.5\right]^{-2}
$$

where Reb is evaluated based on the properties at the local bulk fluid temperature. Although the friction coefficient ratio $\xi_{M} / \xi_{F}$ can be used to describe the approximate trend of the Nusselt number, it is necessary to include the wallto-bulk property ratio to compensate the effect of property variation. To do so, the following wall-to-bulk property ratio terms are adapted to the heat transfer correlation.

$$
\left(\frac{\bar{c}_{p}}{c_{p, b}}\right)^{m}\left(\frac{\rho_{w}}{\rho_{b}}\right)^{n}
$$

Here, $\bar{c}_{p}=\left(H_{w}-H_{b}\right) /\left(T_{w}-T_{b}\right)$, and the exponent $m$ is found to be 0.6 by comparing with experimental data. Finally, the present heat transfer correlation is developed as follows:

$$
\mathrm{Nu}=\mathrm{Nu}_{F}\left(\frac{\xi_{M}}{\xi_{F}}\right)\left(\frac{\bar{c}_{p}}{c_{p, b}}\right)^{0.6}\left(\frac{\rho_{w}}{\rho_{b}}\right)^{n}
$$

where $\mathrm{Nu}_{\mathrm{F}}$ denotes the Dittus-Boelter correlation [1930; Winterton, 1998] developed for the forced convection: it is given by

$$
\mathrm{Nu}_{F}=0.0243 \operatorname{Re}_{b}^{0.8} \operatorname{Pr}_{b}^{0.4}
$$

The index $n$ is considered to be a function of $q / G$, which correlates with experimental results for the small circular tube as follows;

$$
n=0.95546-0.0087089\left(\frac{q}{G}\right)+1.2988 \times 10^{-5}\left(\frac{q}{G}\right)^{2}
$$

The spatially averaged Nusselt number ratio over the heating region, $\overline{N u}_{\exp } / \overline{N u}_{\text {cal }}$ is plotted as a function of $q / G$ in Figure 7-19, where $\overline{N u}_{\text {exp }}$ is evaluated by integrating the local Nusselt numbers over the heated length from all the experimental data for the circular and non-circular tubes, and $\overline{N u}_{c a l}$ is calculated from the correlation given by Equation (7-12). The proposed correlation predicts the average Nusselt number within twenty per cent accuracy for the ninety per cent of present experimental data.

Figure 7-20 shows local Nusselt number variations for the small circular tube in the streamwise direction and the comparisons of the present correlation with present experimental data and other correlations at two different heat fluxes and mass velocities. In the case of $q=50 \mathrm{~kW} / \mathrm{m}^{2}$ and $G=419 \mathrm{~kg} / \mathrm{m}^{2} \mathrm{~s}$ (Figures 7-20(a)), the proposed 
correlation shows an excellent match with the present experimental data. The corresponding Jackson buoyancy parameter Bo McEligot and Jackson [2004], defined as $\mathrm{Gr} /\left(\mathrm{Re}^{3.425} \operatorname{Pr}^{0.8}\right)$, is $1.7 \times 10^{-6}$. This Bo value is about four times larger than that of the case shown in Figures 7-20(b) where $\mathrm{Bo}=4.3 \times 10^{-7}$. At the large Bo value, the present correlation is in relatively good agreement with Kirillov's [2000] correlation compared to other correlations because the flow is in the mixed convection regime. Correlation by Krasnoshchekov and Protopopov [1966] and Jackson [2002] overpredict the Nusselt number at this condition because they are based primarily on forced convection at supercritical pressure and, thus, can hardly account for a heat transfer deterioration due to a buoyancy effect. When the buoyancy effect is weak, as in Figure 7-20(b), the present correlation and experimental data are closer to the correlations of Krasnoshchekov and Protopopov and of Jackson other than the one by Kirillov.

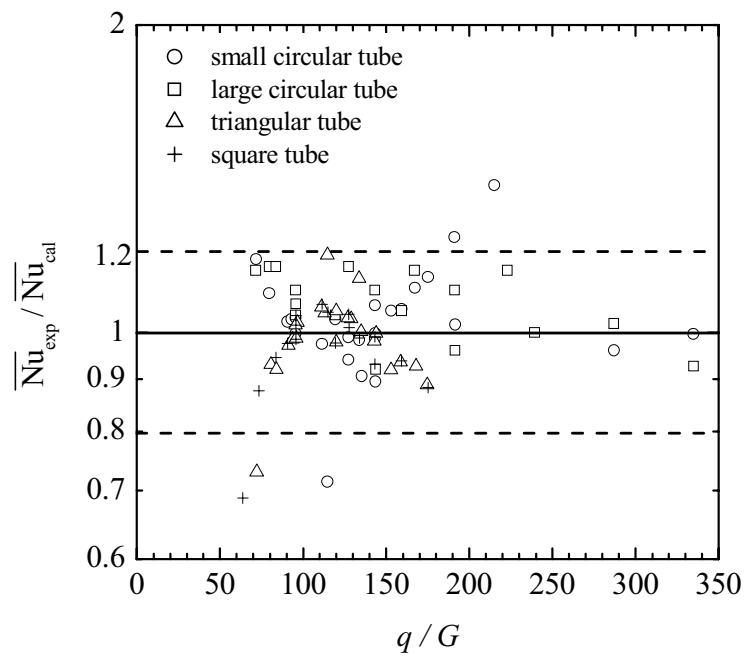

Fig. 7-19. Averaged Nusselt number ratio over the heating region, where $\overline{N u}_{\text {exp }}$ is evaluated from all experimental data for the circular and non-circular tubes, and $N u_{c a l}$ is calculated from the proposed correlation (equations 7-12 to $7-14)$.

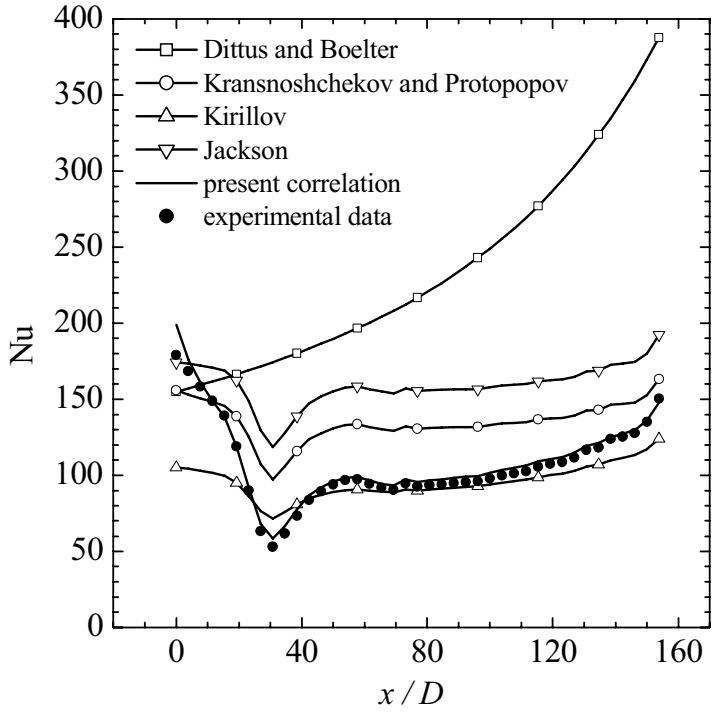

(a)

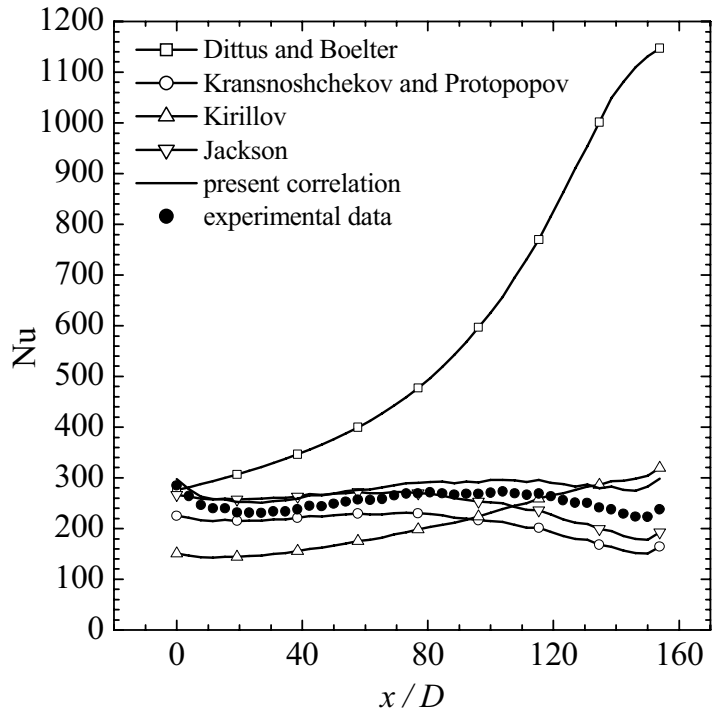

(b)

Fig. 7-20. Local Nusselt number variations for the small circular tube in the streamwise direction, and comparison of the present correlations with present experimental data and other correlations when (a) $q=50 \mathrm{~kW} / \mathrm{m}^{2}, G=419$ $\mathrm{kg} / \mathrm{m}^{2} \mathrm{~s}$ and (b) $q=140 \mathrm{~kW} / \mathrm{m}^{2}, G=837 \mathrm{~kg} / \mathrm{m}^{2} \mathrm{~s}$. 


\section{Hot wire measurements}

The characteristics of heat transfer in turbulent supercritical flow are complicated by large variations in density, specific heat, viscosity and thermal conductivity with pressure and temperature and also by the buoyancy effects. Therefore, measurements of the turbulent quantities are necessary to assess Direct Numerical Simulation (DNS) and Large Eddy Simulation (LES) codes for application to the supercritical flow with heat transfer.

Kurganov and Ankudinov [1986] measured the velocity and temperature profiles of the turbulent supercritical carbon-dioxide flow in the tube by using micro thermocouples and pitot tube. However, from the knowledge of the author, there are no reports on the turbulent quantities measurements of supercritical flow. Therefore, this study is the first attempt to measure the turbulent quantities of supercritical flow by hot wire probe.

\section{Hot wire probe traverse device}

A schematic drawing and photograph of the traverse with its support and probe are shown in Figure 7-21. Since the pressure in the facility is very high and therefore, sealing is a major problem to conduct the experiments with a hot wire probe, it is essential to fabricate the traversing mechanism to avoid leakage during the experiments. In addition to high pressure sealing, the traverse provide electric insulation. Because the traverse retain electric free conditions, it should not contact the test section which is heated by direct current.
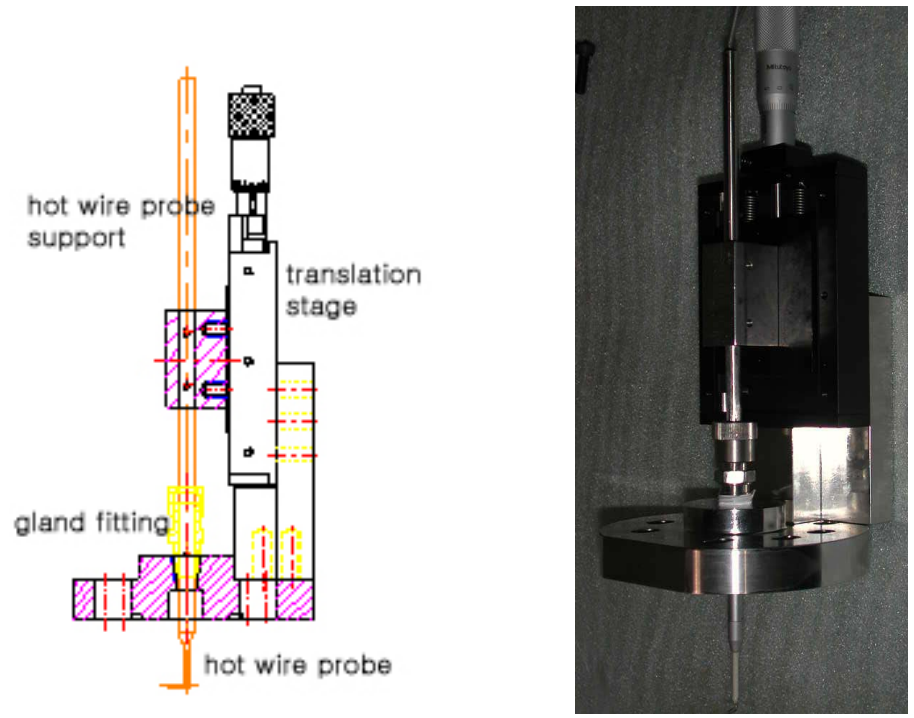

Fig. 7-21. An assembly drawing and photograph of traverse with hot wire support and probe.

The traverse is composed of a translation stage, hot-wire probe support, gland fitting, etc. By rotating the micrometer head of the translation stage, the probe can move forward and backward in the tube with a precision of $0.01 \mathrm{~mm}$ per graduation. The traverse and the calibration test section are assembled by bolts as in Figure 7-22. The hot-wire probe is located at the exit of the jet in the calibration test section to measure the velocity where it has a uniform profile. Therefore, from the measured values of the mass flow rate, inlet fluid bulk temperature and the end area of the jet nozzle, one may calculate the flow velocity. Thus, it is possible to deduce a relationship between the flow velocity and the hot-wire probe signal from the anemometer which is connected to the hot wire probe via the hot-wire probe support.

As mentioned above, electric insulation is essential to the traverse, so, two teflon gaskets and upper / lower plates are manufactured as shown in Figure 7-23 in order to hold the circular tube tightly. Figure 7-24 shows the assembled traverse and the test tube with the upper / lower plates and teflon gaskets. The teflon gaskets are actually placed between the test tube and upper / lower plates and are then pressed securely by bolts to prevent any leakage. A hydraulic pressure test of the test tube and calibration test section were performed successfully without leaks at ten MPa while moving the hot wire probe with the traverse device. 

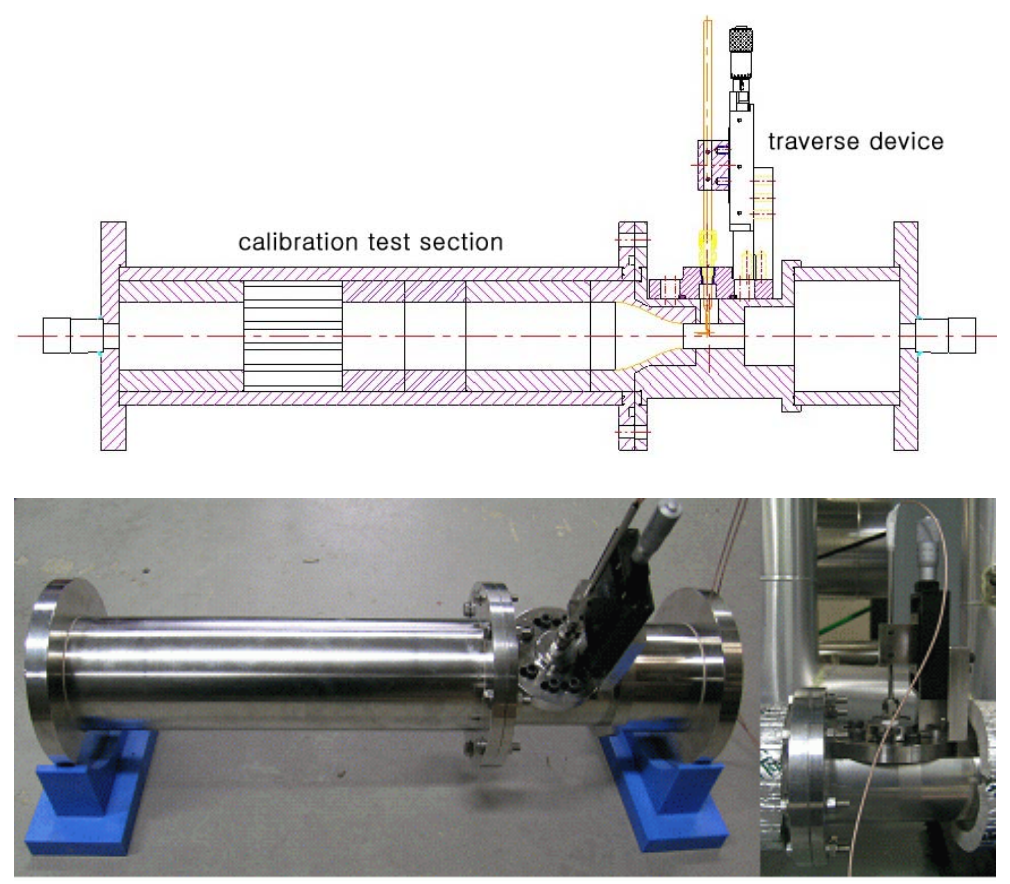

Fig. 7-22. An assembly drawing and photograph of traverse and calibration test section.
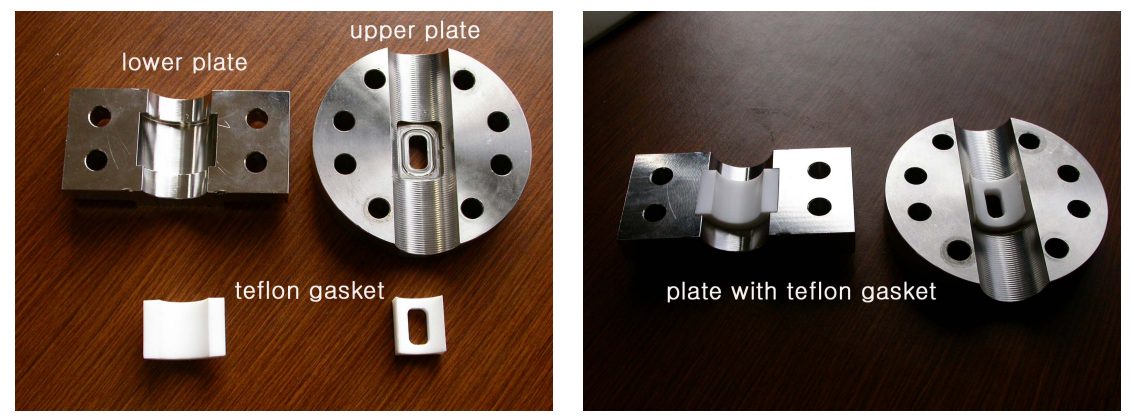

Fig. 7-23. Photographs of upper / lower plates and teflon gaskets to insulate the traversing mechanism from electricity. 

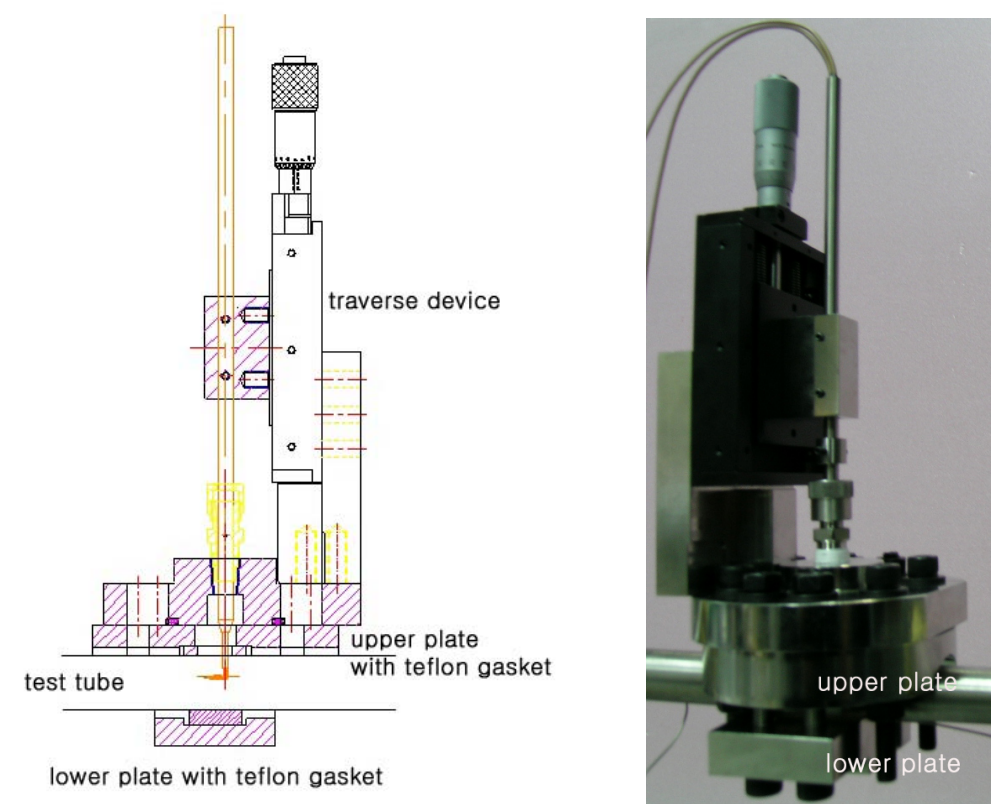

Fig. 7-24. An assembly drawing and photograph of traverse and test section.

A schematic drawing of the test section for measurements of turbulent quantities with hot-wire probe is shown in Figure 7-25. The test section is the same as that already used for the large circular tube experiment. Thus, the diameter of the test tube is $19.4 \mathrm{~mm}$ with a thickness of $0.8 \mathrm{~mm}$. The measuring location of the traverse is fixed as in Figure 7-25, $1.7 \mathrm{~m}$ from the starting point of the entrance region. To measure turbulent quantities at several locations along the axial direction where meaningful heat transfer phenomena would occur, it is useful to shift the heating region by varying the entrance length by moving the two electrode positions (rather than installing several traverses along the test section). The entrance length can be varied from a minimum of $0.58 \mathrm{~m}$ to a maximum of $1.64 \mathrm{~m}$. In this way, we can obtain the same wall temperature distributions at any heating region following an entrance region in the test section. Therefore, in spite of the fixed location of the traverse, we can measure turbulent quantities at any positions we want.

Hot wire probe calibration

The calibration loop shown in Figure 7-26 is constructed by placing the hot-wire calibration section into the test facility. Thus, the preheater, flowmeter, pump, etc. are used for the calibration and measurements at the test as shown in Figure 7-27. We use a Dantec I-type hot-wire probe Model $55 \mathrm{P} 14$ because it is compatible with the existing equipment such as a hot-wire welder and anemometer. The wire material is tungsten and the length and the diameter are $1.25 \mathrm{~mm}$ and $5 \mu \mathrm{m}$, respectively. 


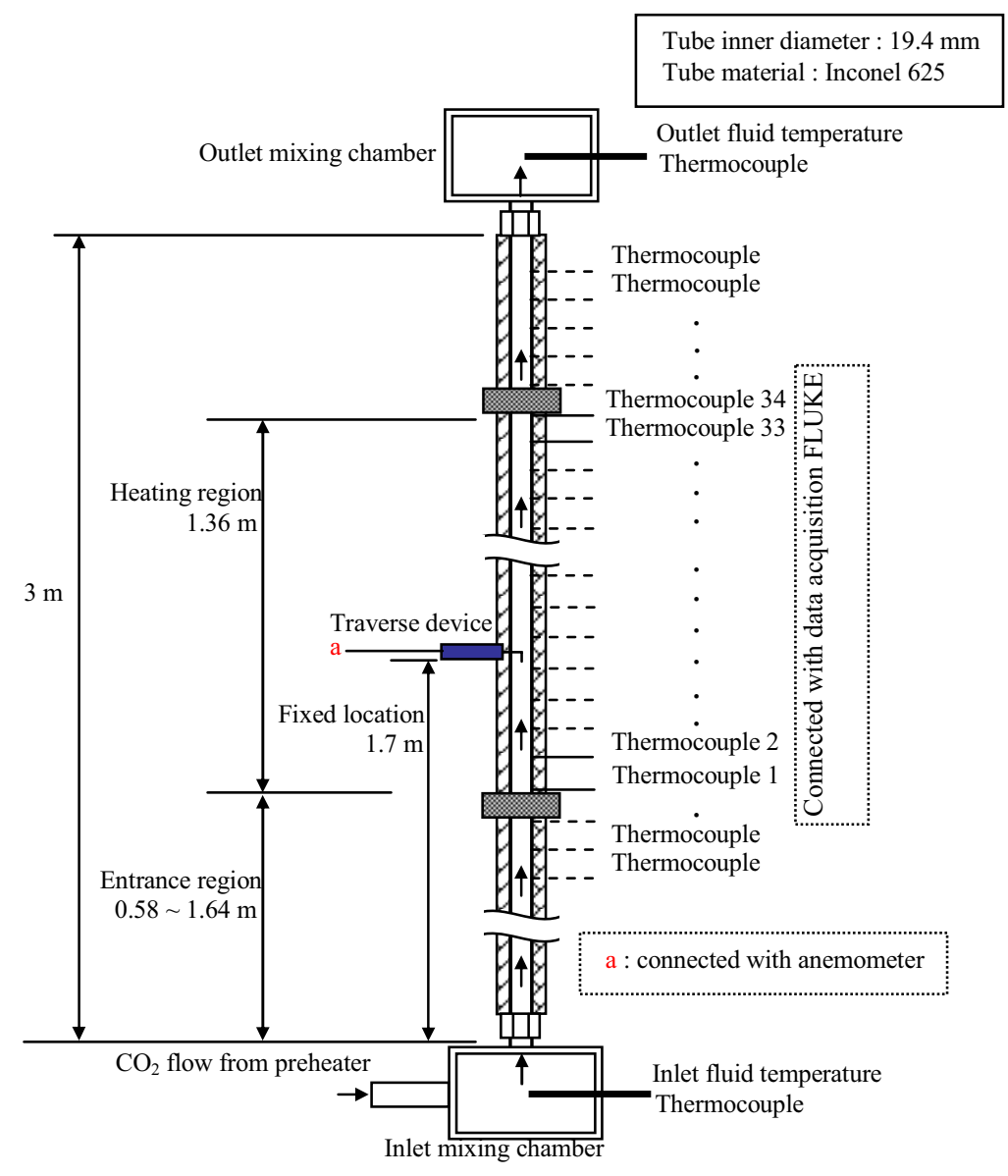

Fig. 7-25. Schematic drawing of test section for the measurements of turbulent quantities using a hot-wire probe. 

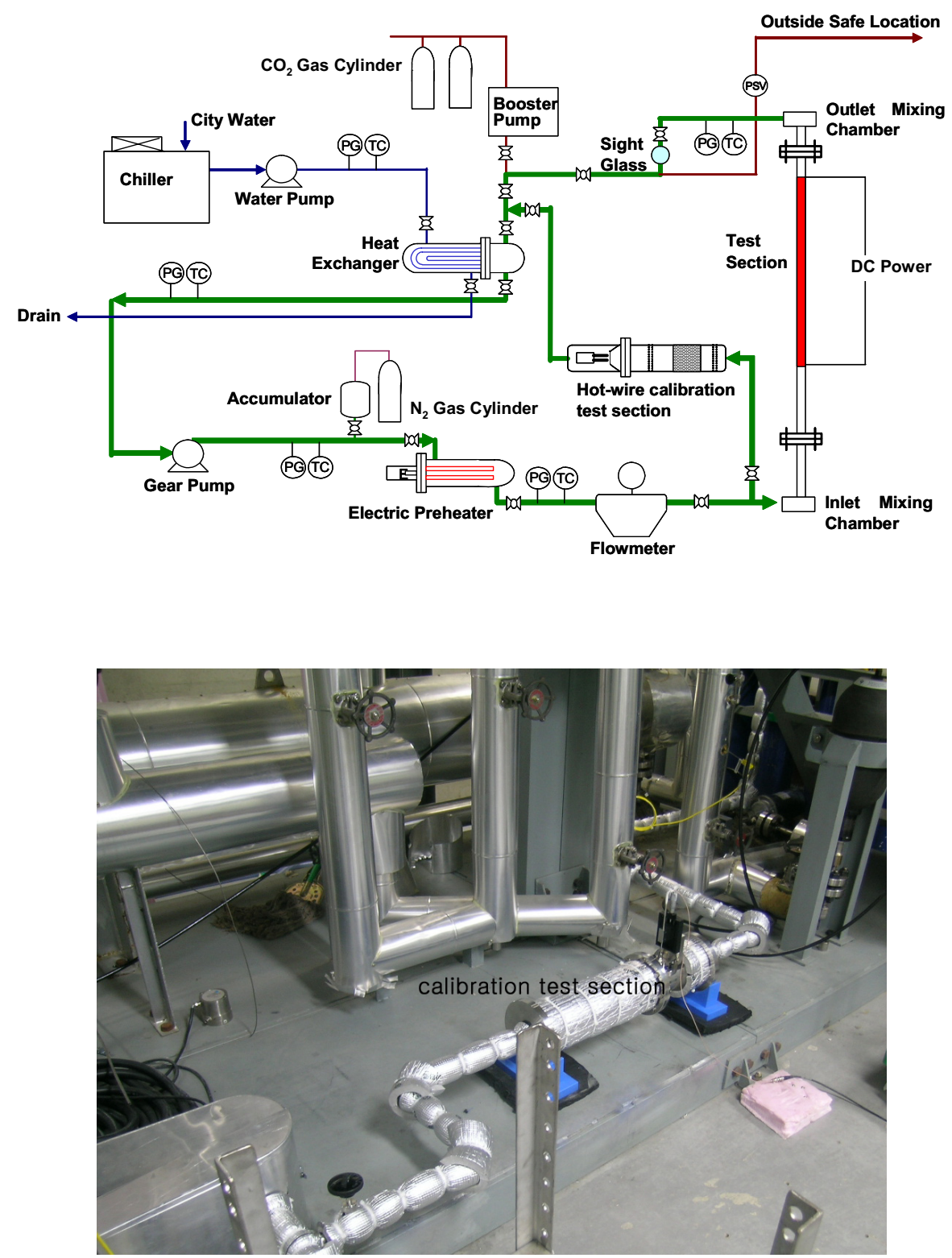

Fig. 7-26. Schematic drawing and photograph of hot-wire probe calibration loop.

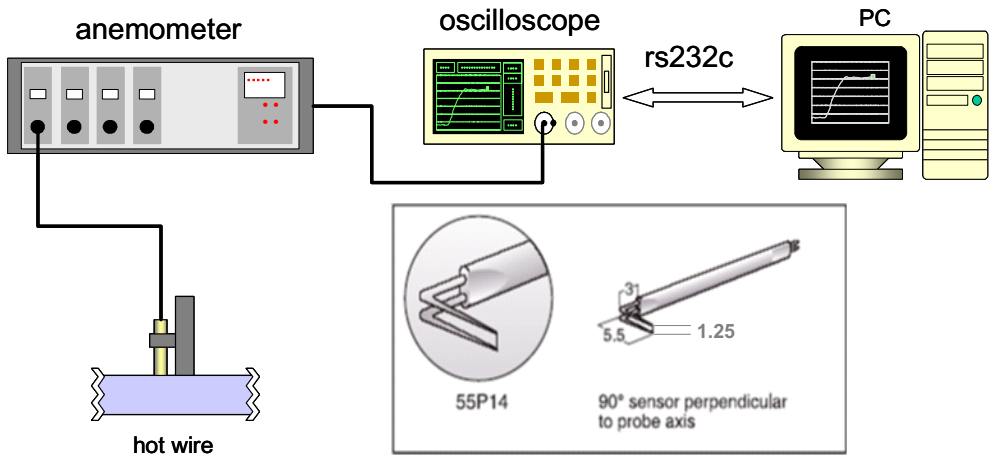

Fig. 7-27: Data acquisition system for hot-wire measurements. 


\section{Hot wire probe calibration results}

The velocity calibration for supercritical carbon-dioxide flow in the fluid temperature range from 15 to $40 \mathrm{C}$ is shown in Figure 7-28. The flow velocity varies from about 0.3 to $0.5 \mathrm{~m} / \mathrm{s}$. The data are obtained in the constant temperature mode with the wire with the wire at $80 \mathrm{C}$. The experimental conditions are listed in Table 7-8.

Figure 7-29 shows the temperature calibration. The calibration signal at a constant fluid temperature shows a constant value with regardless of the flow velocity, as in Figure 7-29(a). Therefore, as shown in Figure 29(b), there is one calibration curve for the fluid temperature range irrespective of the flow velocity.

We continue the calibrations to obtain more reliable data since output voltages for the velocity measurements do not show sufficient repeatability. After we obtain reproducibility, we can begin measurement of the turbulent quantities.

Table 7-8. Experimental conditions for the hot wire probe calibration.

Pressure $[\mathrm{MPa}] \quad 8$

Fluid temperature $\left[{ }^{\circ} \mathrm{C}\right] \quad 15,20,25,30,32,38,40$

Velocity $[\mathrm{m} / \mathrm{s}]$ $0.3,0.4,0.5$

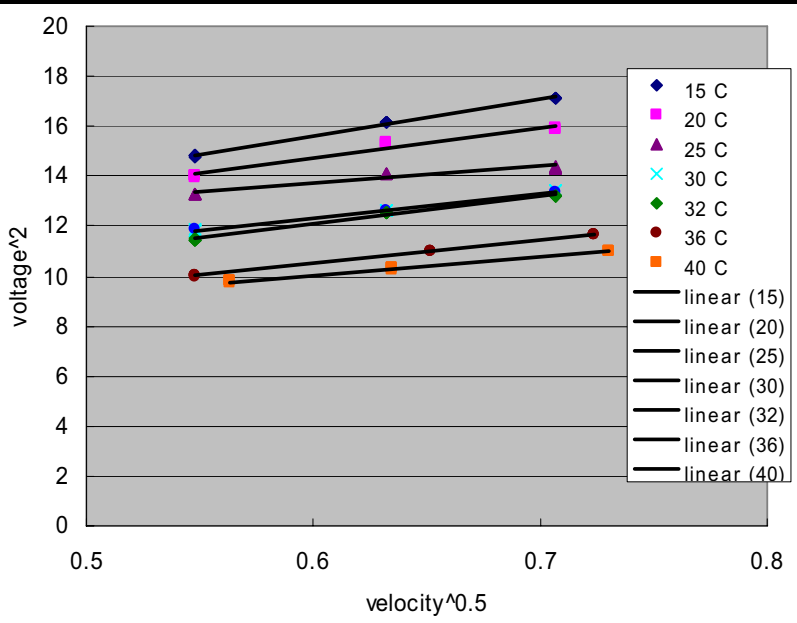

Fig. 7-28. Velocity calibration for hot-wire in the fluid temperature range from 15 to $40 \mathrm{C}$ and velocity range from 0.3 to $0.5 \mathrm{~m} / \mathrm{s}$.

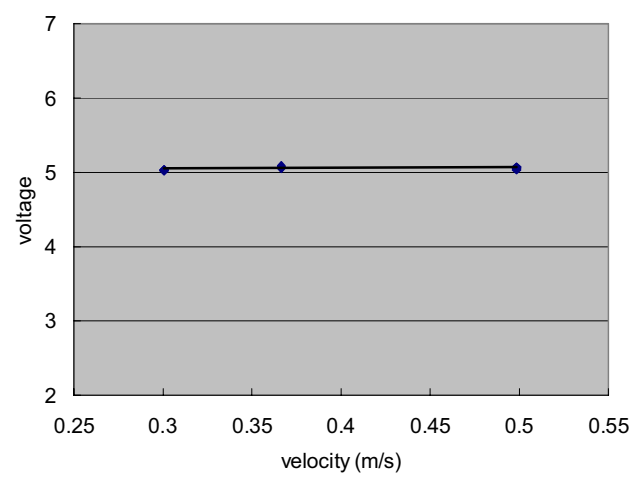

(a)

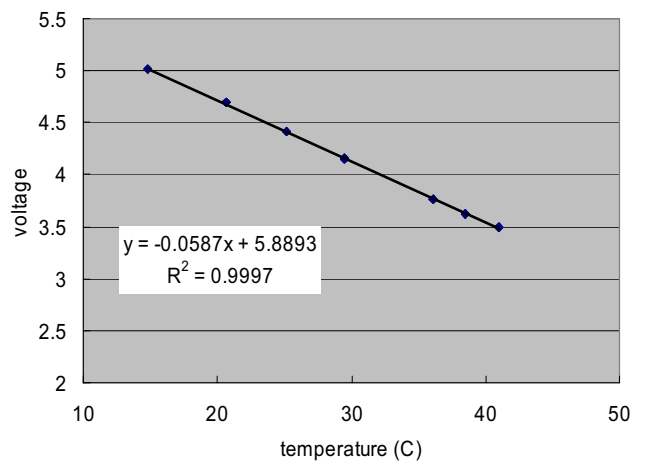

(b)

Fig. 7-29. Temperature calibration curve: (a) velocity variation at a constant fluid temperature of $15 \mathrm{C}$ and (b) fluid temperature variation from 15 to $40 \mathrm{C}$. 


\section{Uncertainty analysis}

The uncertainty analysis for this experiment is conducted according to the methods of Holman [2001]. Prior to calculating the uncertainty of Nusselt number, the uncertainty of wall temperature measurements and fluid temperature calculation are investigated. The wall temperatures measured by thermocouples contain uncertainties due to the heat transfer along the thermocouple at the measuring point. Therefore, the actual wall temperature is higher than the measured value. For these and other reasons, we consider the uncertainty of fluid and wall temperature to calculate the uncertainty of the Nusselt number.

From Equation (7-1), one may express the uncertainty of the fluid enthalpy at any axial location $x$ as follows.

$$
\text { Uncertainty }=\left[\left(\frac{\partial H_{x}}{\partial H_{i}} \omega_{H_{i}}\right)^{2}+\left(\frac{\partial H_{x}}{\partial q^{\prime \prime}} \omega_{q^{\prime \prime}}\right)^{2}+\left(\frac{\partial H_{x}}{\partial \dot{m}} \omega_{\dot{m}}\right)^{2}\right]^{1 / 2}
$$

The uncertainty of heat flux imposed to the test section is about one per cent and for mass flow rate is 0.5 per cent. The inlet temperature error of 0.02 per cent is calculated from the 0.1 per cent uncertainty of the temperature indicator. With these estimates, the uncertainty of fluid enthalpy is 0.28 per cent for a typical experiment with mass velocity of $0.02 \mathrm{~kg} / \mathrm{s}$ and heat flux of $50 \mathrm{~kW} / \mathrm{m}^{2}$. However, we can also obtain a check of the fluid enthalpy by direct comparison between the calculated fluid temperature and the measured outlet temperature. From the overall experimental results, these differences for fluid temperature and related enthalpy indicate about 3.7 per cent and 3.6 per cent, respectively. As in the case of the fluid temperature near the pseudocritical temperature, the uncertainty of enthalpy is high in spite of little difference of the fluid temperatures between the calculated and measured one because the properties change abruptly near this temperature. Thus, we choose the uncertainty of the fluid temperature to be about 3.7 per cent and use this value to estimate the uncertainty in Nusselt number.

When thermocouples are used to measure the temperatures of surfaces which are exposed to fluids at temperatures different from those of the surfaces, errors occur because of thermal conduction along the thermocouple wires. Beyond the insulation surrounding the test section, the thermocouple wires used in this experiment are exposed to ambient medium whose temperature is slightly lower than that of test section surface. Thus, the wall temperature recorded by thermocouple is lower than the actual value. Therefore, we calculate the real wall temperature by considering the heat loss from the thermocouple wire to the ambient surroundings due to radiation and convection effects and find a "worst case" thermocouple conduction error. To do this we adopted the Equation (7-16) of Schneider [1955] and Hess, Deardorff and McEligot [1971]. The heat transfer equation for thermocouple wire attached to a test section is as follows.

$$
\begin{gathered}
\frac{T_{T C}-T_{w u}}{T_{w u}-T_{\infty}}=\frac{\left(h_{o}-h_{T C}\right) r K_{o}(\lambda r)}{\left(h_{i}+h_{T C}\right) r K_{o}(\lambda r)+2 \lambda k_{w} \delta K_{1}(\lambda r)} \\
h_{T C}=\frac{k_{T C}\left[\frac{2 \beta}{5}\left(T_{T C}^{5}-5 T_{T C} T_{\infty}^{4}+4 T_{\infty}^{5}\right)+\frac{2 h}{k_{T C} r}\left(T_{T C}^{2}-2 T_{T C} T_{\infty}+T_{\infty}\right)\right]^{1 / 2}}{T_{T C}-T_{\infty}}
\end{gathered}
$$

The value of $T_{T C}$ is the wall temperature recorded by thermocouple and $T_{w u}$ is the desired wall temperature in the absence of thermocouple. Therefore, the difference $\left(T_{T C}-T_{w u}\right)$ is the thermocouple temperature error. Here, the $h_{T C}$ is the effective heat transfer coefficient of thermocouple. The quantities $h_{o}$ and $h_{i}$ are the convective heat transfer coefficients for (1) thermocouple surface to the ambient and (2) the inner wall to the carbon dioxide, respectively. The quantity $h_{i}$ can be calculated from the experimental results and $h_{o}$ is explained later. The radius $r$ is the effective value $(2 \mathrm{~mm})$ at the of attachment point of the thermocouple. $K_{o}$ and $K_{l}$ are the modified Bessel functions of second kind, zero and first order, respectively. The quantity $\beta$ is a heat transfer constant expressed as $2 \sigma \varepsilon /\left(k_{T C} r\right)$, where $\sigma$ is the Stefan-Boltzmann constant $\left(5.67051 \times 10^{-8} \mathrm{~W} / \mathrm{m}^{2} \mathrm{~K}^{4}\right), \varepsilon(0.87)$ is the thermal emissivity of the thermocouple and $\delta$ is the thickness $(1 \mathrm{~mm})$ of the test section. Above mentioned $h_{o}$ can be obtained from the Nusselt number correlation expressed as in Equations (7-18 and 7-19) for mixed convection flow over a horizontal 
cylinder by approximating that thermocouple as a cylinder. These equations can be applied for the conditions of $1<$ $\operatorname{Re}_{\mathrm{d}}<60$ and $0<\mathrm{Gr}_{\mathrm{d}}<7200$,

$$
\begin{gathered}
\frac{N u_{d}}{N u_{d F}}=1+0.16 \frac{G r_{d}}{\operatorname{Re}_{d}^{2}}-0.015\left(\frac{G r_{d}}{\operatorname{Re}_{d}^{2}}\right)^{2} \\
N u_{d F}=1.01+9.1 \times 10^{-2} \operatorname{Re}_{d}-7.3 \times 10^{-4} \mathrm{Re}_{d}^{2}
\end{gathered}
$$

where $N u_{d F}$ and $N u_{d}$ are the Nusselt numbers for forced and mixed convection, respectively. For a given ambient temperature, thermocouple wire temperature, and air velocity in the laboratory, the average thermocouple conduction error is estimated to be about six per cent with a maximum value of eight per cent for the experimental conditions with mass velocity of $419 \mathrm{~kg} / \mathrm{s}$ and heat flux of $50 \mathrm{~kW} / \mathrm{m}^{2}$.

From Equation (7-15), we can estimate the uncertainty of Nusselt number as follows

$$
\text { Uncertainty }=\left[\begin{array}{l}
\left(\frac{\partial N u}{\partial q^{\prime \prime}} \omega_{q^{\prime \prime}}\right)^{2}+\left(\frac{\partial N u}{\partial d} \omega_{d}\right)^{2}+\left(\frac{\partial N u}{\partial k} \omega_{k}\right)^{2} \\
+\left(\frac{\partial N u}{\partial T_{w}} \omega_{T_{w}}\right)^{2}+\left(\frac{\partial N u}{\partial T_{b}} \omega_{T_{b}}\right)^{2}
\end{array}\right]^{1 / 2}
$$

We neglect the effect of a diameter error. The average uncertainty of Nusselt number is estimated to be about ten per cent with a maximum value of about eleven per cent. This uncertainty is dominated by the uncertainty in the thermocouple conduction error. 


\title{
Appendix. Scholarly accomplishments of partners of KNERI project 2002-016-K
}

\author{
Advanced computational thermal fluid physics (CTFP) and its assessment for \\ light water reactors and supercritical reactors
}

\begin{abstract}
Awards and recognition
Prof. James M. Wallace of the University of Maryland became Chairman of the Fluid Dynamics Division of the American Physical Society in November 2003. Prof. Jung Yul Yoo was chosen as the 2003 Distinguished Professor in Research by the College of Engineering at Seoul National University. The Principal Investigator, Prof. Dr. Donald M. McEligot, received from the Idaho Academy of Science its annual award as their Distinguished Scientist for 2002 "for his pioneering experiments and analyses in convective thermal fluid physics and for his technical leadership in developing the World's largest Matched-Index-of-Refraction system to study complex flow phenomena."

Prof. Jung Yul Yoo is a Member of the National Academy of Engineering of Korea; he was selected as a Fellow of the American Society of Mechanical Engineers in June 2002 and was elected to the Korean Academy of Science and Technology in November 2002. He served through March 2003 as Secretary General of the Korea Research Foundation, an organization affiliated with the Ministry of Education and Human Resources Development of the Republic of Korea. Currently Prof. Yoo is President of the Korean Society of Theoretical and Applied Mechanics and is Vice-President of the Korean Society of Mechanical Engineers. He is also President of the Korean Society of Visualization and Vice-President of the Biomedical Engineering Society for Circulatory Disorders. Prof. Joon Sik Lee is a member of the Executive Committee of the International Centre of Heat and Mass Transfer. In 2002 he served as Assistant to the President, Prof. Sung Tack Ro, for the Assembly for International Heat Transfer Conferences.
\end{abstract}

\section{Publications and presentations relating to collaborative KNERI project}

\section{Archival publications}

Kim, W. S., C. Talbot, B. J. Chung and J. D. Jackson, 2002. Mixed convection heat transfer with variable properties to air flowing through a vertical passage of annular cross section. Trans., Institution of Chemical Engineers Part A, Chemical Engineering Research and Design, 80, Part A, pp. 239-245.

Wu, T. H., Z. Xu and J. D. Jackson, 2002. Mixed convection heat transfer to water flowing through a vertical passage of annular cross section. Trans., Institution of Chemical Engineers Part A, Chemical Engineering Research

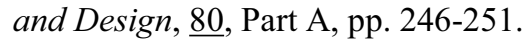

Wang, J., J. Li and J. D. Jackson, 2002. Mixed convection heat transfer to air flowing upwards through a vertical plane passage. Trans., Institution of Chemical Engineers Part A, Chemical Engineering Research and Design, $\underline{80}$, Part A, pp. 252-260.

Jackson, J. D., J. Li and J. Wang, 2002. The effects of buoyancy on turbulent flow and heat transfer in a vertical plane passage. Advances in Turbulence IX, (Ed.: I.P. Castro, P.E. Hancock and T.G. Thomas).

Jackson, J. D., T. Wu and Z. Xu, 2002. Turbulent, buoyancy-influenced flow and heat transfer in a vertical annulus. Heat Transfer 2002, Proc., 12th Int. Heat Transfer Conf., Grenoble, August. 
Jackson, J. D., and J. Li, 2002. Influences of buoyancy and thermal boundary conditions on heat transfer with naturally-induced flow in a tube. Heat Transfer 2002, Proc., 12th Int. Heat Transfer Conf., Grenoble, August.

Mikielewicz, D. P., A. M. Shehata, J. D. Jackson and D. M. McEligot, 2002. Temperature, velocity and mean turbulence structure in strongly-heated internal gas flows. Comparison of numerical predictions with data. Int. J. Heat Mass Transfer, 45, pp. 4333-4352.

Vukoslavcevic, P., and J. M. Wallace, 2002. The simultaneous measurement of velocity and temperature in heated turbulent air flow using thermal anemometry. Meas. Sci. Technol. 13, pp. 1615-1624.

Dailey, L. D., N. Meng and R. H. Pletcher, 2003. Large eddy simulation of constant heat flux turbulent channel flow with property variations: Quasi-developed model and mean flow results. J. Heat Transfer, $\underline{125}$, pp. 27-38.

Jackson, J. D., 2003. Influences of buoyancy on velocity, turbulence and heat transfer in ascending and decending flows in vertical passages. Adv. Heat Transfer Engr. (Ed.: B. Junden and J. Vilemas), New York: Begel House.

You, J., J. Y. Yoo and H. Choi, 2003. Direct numerical simulation of heated vertical air flows in fully developed turbulent mixed convection. Int. J. Heat Mass Transfer, 46 (9), pp. 1613-1627.

Moro, J. P., P. V. Vukoslavcevic and V. Blet, 2003. A method to calibrate a hot-wire X-probe for applications in low-speed, variable temperature flow. Meas. Sci. Technology, 14, pp. 1054-1062.

Kim, W. S., J. D. Jackson and S. He, 2003. Comparative study of turbulence models applied to buoyancy-opposed convection in a vertical passage. Turbulence, Heat Mass Transfer (Ed.: K. Hanjalic, Y. Nagano and M. J. Tummers), New York: Begell House.

Jackson, J. D., Z. Xu and T. H. Wu, 2003. On the influence of buoyancy on turbulence production and effectiveness of heat transfer in vertical flows. Turbulence, Heat Mass Transfer (Ed.: K. Hanjalic, Y. Nagano and M. J. Tummers), New York: Begell House.

Petrovic, D., P. Vukoslavcevic and J. M. Wallace, 2003. The accuracy of turbulent velocity component measurements by multi-sensor hot-wire probes: a new approach to an old problem. Exp. Fluids, $\underline{34}$, pp. 130-139.

Baek, S. G., and S. O. Park, 2003. Assessment of explicit algebraic stress/heat-flux models for reduction of heat transfer in a vertical pipe with intense heating. Transactions of the Korean Society of Mechanical Engineers Part B, $\underline{27}$, No. 15, pp. 1724-1733 (in Korean).

Spall, R. E., A. H. Richards and D. M. McEligot, 2004. An assessment of the k- $\omega$ and $\mathrm{v}^{2}$-f turbulence model for strongly heated internal gas flows. Numerical Heat Transfer, Part A, 46, pp. 831-849.

Lee, J. S., X. Xu and R. H. Pletcher, 2004. Large eddy simulation of heated vertical annular pipe flow in fully developed turbulent mixed convection. Int. J. Heat Mass Transfer, 47, pp. 437-446.

McEligot, D. M., and J. D. Jackson, 2004. "Deterioration" criteria for convective heat transfer in gas flow through non-circular ducts. Nuc. Engr. Design, 232, pp. 327-333.

Xu, X., J. S. Lee, R. H. Pletcher, A. M. Shehata and D. M. McEligot, 2004. Large eddy simulation of turbulent forced gas flows in vertical pipes with high heat transfer rates. Int. J. Heat Mass Transfer, 47, pp. 4113-4123.

Wang, J., J. Li and J. D. Jackson, 2004. A study of the influence of buoyancy on turbulent flow in a vertical plane passage. Int. J. Heat Fluid Flow, 25 (Special issue), pp. 420-430,

He, S., W. S. Kim, P. X. Jiang and J. D. Jackson, 2004. Simulation of mixed convection heat transfer to carbon dioxide at supercritical pressure. Int. J. Mech. Eng. Sci., Proc. I. Mech. E., 218, Part C, Special Issue, pp. 12811296. 
Kim, W. S., J.D. Jackson and S. He, 2004. Variable property buoyancy-influenced heat transfer to air flowing in a vertical passage of annular cross-section. J. Mech. Eng. Sci., in press.

Wang, J., J. Li, S. He and J. D. Jackson, 2004. Computational simulations of buoyancy-influenced turbulent flow and heat transfer in a vertical plane passage. Int. J. Mech. Eng. Sci., Proc. I. Mech. E., 218, Part C, Special Issue, pp. 1385-1397.

Kim, W. S., J. D. Jackson, S. He and J. Li, 2004. Performance of a variety of low Reynolds number turbulence models applied to mixed convection heat transfer to air flowing in a vertical tube. Int. J. Mech. Eng. Sci., Proc. I. Mech. E., 218, Part C, Special Issue, pp. 1361-1373.

Xu, X., J. S. Lee and R. H. Pletcher, 2005. A compressible finite volume formulation for large eddy simulation of turbulent pipe flows at low Mach number in Cartesian coordinates. J. Computational Physics, 203, pp. 22-48

Bae, J. H., J. Y. Yoo, H. Choi and J. You, 2004. Direct numerical simulation of strongly-heated internal gas flows with large variations of fluid properties. Transactions of the Korean Society of Mechanical Engineers Part B, 28, No. 11, pp. 1289-1301 (in Korean).

Bae, J. H., J. Y. Yoo and H. Choi, 2004. Direct numerical simulation of turbulent heat transfer to fluids at supercritical pressure flowing in vertical tubes. Transactions of the Korean Society of Mechanical Engineers Part $B, \underline{28}$, No. 11, pp. 1302-1314 (in Korean).

Vukoslavcevic, P., D. Petrovic and J. M. Wallace, 2004. An analytical approach to the uniqueness problem of hotwire probes to measure simultaneously three velocity components. Meas. Sci. Technol., 15, pp. 1848-1854.

He, S., P.X. Jiang, Y. J. Xu, R. F.Shi, W. S. Kim and J.D. Jackson, 2005. A computational study of convective heat transfer of $\mathrm{CO}_{2}$ at supercritical pressure in a vertical mini tube. International Journal of Thermal Sciences, 44, pp. 521-530.

Bae, J. H., J. Y. Yoo and H. Choi. 2005. Direct numerical simulation of turbulent supercritical flows with heat transfer. Physics of Fluids, in press.

Baek, S. G., and S. O. Park, 2005. Effect of wall distance coordinate on the prediction of variable property flow with two-equation turbulence model. International Journal of Computational Fluid Dynamics, in press.

Vukoslavcevic, P.V., I. Radulovic and J. M. Wallace, 2005. Testing of hot and cold-wire probe to measure simultaneously the speed and temperature in supercritical CO2 flow. Experiments in Fluids, in press.

\section{Conference publications and presentations}

Jackson, J. D., and W. S. Kim, 2002. Computational modeling of mixed convection experiments - gas cooled reactors. Universities Nuclear Technology Forum, Lancaster, UK, April 10-12.

Jackson, J. D., and Z. Xu, 2002. Measurements of buoyancy-influenced turbulent flow for validating CFD codes used in nuclear reactor applications. Universities Nuclear Technology Forum, Lancaster, UK, April 10-12.

Jackson, J. D., J. Li and J. Wang, 2002. The effects of buoyancy on turbulent flow and heat transfer in a vertical plane passage. Euromech 9th European Turbulence Conference, Southampton, UK, July 2-5.

Satake, S., T. Kunugi, A. M. Shehata and D. M. McEligot, 2002. DNS of forced gas flows in circular tubes at various heating rates. Presentation OFS 12, 12th International Heat Transfer Conference, Grenoble, Aug.

Jackson, J. D., 2002. Consideration of the heat transfer properties of supercritical pressure water in relation to its use as a coolant in advanced nuclear reactors. 13th Pacific Basin Nuclear Conference, Shenzhen, China, Oct. 21-25. 
Xu, X., J. S. Lee and R. H. Pletcher, 2002. Cartesian-based finite volume formulation for LES of mixed convection in a vertical turbulent pipe flow. ASME Int. Mechanical Engineering Congress, Paper IMECE2002-HT-32748, New Orleans, Nov.

Lee, J. S., X. Xu and R. H. Pletcher, 2002. Large eddy simulation of mixed convection in a vertical annular pipe flow. ASME International Mechanical Engineering Congress, Paper IMECE2002-HT-32746, New Orleans, Nov.

McCreery, G. E., T. D. Foust, D. M. McEligot, K. G. Condie and R. J. Pink, 2002. Measurements and code comparisons for advanced gas reactor coolant channels containing spacer ribs. ASME International Mechanical Engineering Congress, Paper IMECE2002-33597, New Orleans, Nov.

Vukoslavcevic, P., and J. M. Wallace, 2002. A method to determine the time constant of a cold-wire temperature sensor. 55th Annual Meeting, American Physical Society Division of Fluid Dynamics, Dallas, Nov. (also Bull., Amer. Phys. Soc., 47, [10], p. 148.)

Yoo, J. Y., 2003. Direct numerical simulation of heat transfer to $\mathrm{CO}_{2}$ at supercritical pressure in a vertical tube. Workshop on Supercritical Reactors, pp. 155-199, Seoul, Korea, March 23-24.

Wang, J., J. K. Li and J. D. Jackson, 2003. Influence of buoyancy on turbulent air flow in a vertical plane passage. 3rd International Symposium on Turbulence and Shear Flow Phenomena (TSFP-3), Sendai, Japan, June 25-27.

Chung, W. S., O. Kwon, S. Park, Y. K. Choi and J. S. Lee, 2003. Tunable AC thermal anemometry. Microscale Heat Transfer 2, Eurotherm Seminar, (75), pp. 51-52, Reimes, France, July 8-10.

Jackson, J. D., 2003. Influences of buoyancy on velocity, turbulence and heat transfer in ascending and decending flows in vertical passages (Keynote lecture). 4th Baltic Heat Transfer Conf., Kanuas, Lithuania, Aug. 25-27.

Jackson, J. D., K. O. J. Evans Lotterodt, R. Weinberg, J. Fewster and D. P. Mikielewicz, 2003. Experimental and computational modelling studies of mixed convection heat transfer to liquid-like carbon dioxide at supercritical pressure. 4th Baltic Heat Transfer Conf., Kanuas, Lithuania, Aug. 25-27.

Moon, C. M., S. G. Baek and S. O. Park, 2003. Assessment of two-equation turbulence models in FLUENT for a turbulent heated pipe flow. Korean Society of Computational Fluids Engineering Conference, pp.158-163, Daegu, Korea, Aug. 29 (in Korean).

Wang, J., J. Li, S. He and J. D. Jackson, 2003. Computational simulations of buoyancy-influenced turbulent flow and convective heat transfer in a vertical plane passage. UK National Heat Transfer Conf., Oxford, UK, 9-10 September.

Kim, W. S., S. He, J. Li and J. D. Jackson, 2003. Performance of a variety of low Reynolds number turbulence models applied to mixed convection heat transfer to air flowing in a vertical tube. UK National Heat Transfer Conf., Oxford, UK, 9-10 September.

Xu, Z., S. He, T. H. Wu and J. D. Jackson, 2003. Comparisons between measurements of velocity, turbulence and temperature in buoyancy-influenced flow in a passage of annular cross section with computational simulations. UK National Heat Transfer Conf., Oxford, UK, 9-10 September.

Jiang, P. X., Y.J . Xu, J. Lu, H. Xiang and J. D. Jackson, 2003. Experimental investigation of convection heat transfer to $\mathrm{CO}_{2}$ at super-critical pressures in vertical mini tubes and in porous media. UK National Heat Transfer Conf., Oxford, UK, 9-10 September.

He, S., W. S. Kim, P. X. Jiang and J. D. Jackson, 2003. Mixed convection heat transfer to carbon dioxide at supercritical pressure comparisons between experiments and computational simulations using advanced turbulence models. UK National Heat Transfer Conf., Oxford, UK, 9-10 September. 
Jackson, J. D., K. O. J. Evans Lutterodt, R. Weinberg and J. Fewster, 2003. Experimental studies of buoyancyinfluenced convective heat transfer in heated vertical tubes at pressures just above and just below the thermodynamic critical value. GENES4/ANP2003, Kyoto, Japan, Sept.

McCreery, G. E., J. Buongiorno, K. G. Condie, D. M. McEligot, M. E. Nitzel and J. E. O'Brien, 2003. The INEEL heat transfer flow loop for development of supercritical-pressure water reactors (SCWRs). GENES4/ANP2003, Kyoto, Japan, Sept. 15-19.

McCreery, G. E., R. J. Pink, K. G. Condie and D. M. McEligot, 2003. Fluid dynamics of ribbed annuli. Paper J00203, NuReTH-10, Seoul, Oct.

Kim, W. S., J. D. Jackson and S. He, 2003. Variable property buoyancy-influenced heat transfer to air flowing in a vertical passage of annular cross-section. Paper A00114, NuReTH-10, Seoul, Oct.

Baek, S. G., and S. O. Park, 2003. Prediction of strongly-heated gas flows in a vertical tube using explicit algebraic stress/heat-flux models. Paper E00212, NuReTH-10, Seoul, Oct.

Bae, J. H., J. Y. Yoo, H. Choi, 2003. Direct numerical simulation of heat transfer to $\mathrm{CO}_{2}$ at supercritical pressure in a vertical tube. Paper A00112, NuReTH-10, Seoul, Oct.

Kim, W. S., J. D. Jackson and S. He, 2003. Comparative study of turbulence models applied to buoyancy-opposed convection in a vertical passage. 4th Int. THMT, Antalya, Turkey, Oct. 12-17.

Jackson, J. D., Z. Xu and T. H. Wu, 2003. On the influence of buoyancy on turbulence production and effectiveness of heat transfer in vertical flows. 4th Int. THMT, Antalya, Turkey, Oct. 12-17.

Lee, J. S., X. Xu and R. H. Pletcher, 2003. LES of compressible turbulent annular pipe flow with a rotating inner wall. Paper IMECE2003-41841, ASME Int. Mech. Eng. Congress, Nov.

Bae, J. H., J. Y. Yoo and H. Choi, 2003. Direct numerical simulation of strongly-heated internal gas flows with large variations of fluid properties. 56th Annual Mtg., American Physical Society Division of Fluid Dynamics, East Rutherford, N. J., Nov. (also Bull., Amer. Phys. Soc., $\underline{48}$, [10], p. 22.)

Vukoslavcevic, P., and J. M. Wallace, 2003. A hot-wire probe to simultaneously measure velocity vector and temperature fields. 56th Annual Mtg., American Physical Society Division of Fluid Dynamics, East Rutherford, N. J., Nov. (also Bull., Amer. Phys. Soc., $\underline{48}$, [10], p. 95-96.)

Park, N., J. Y. Yoo and H. Choi, 2003. Toward consistent a priori and a posteriori tests in large eddy simulation. 56th Annual Mtg., American Physical Society Division of Fluid Dynamics, East Rutherford, N. J., Nov. (also Bull., Amer. Phys. Soc., $\underline{48},[10]$, pp. 54-55.)

Baek, S. G., and S. O. Park, 2003. Comparative study of turbulence models in predicting the mixed convection flow under supercritical pressure. 56th Annual Mtg., American Physical Society Division of Fluid Dynamics, East Rutherford, N. J., Nov. (also Bull., Amer. Phys. Soc., $\underline{48}$, [10], p. 37-38.)

Richards, A. H., R. E. Spall and D. M. McEligot, 2004. An assessment of turbulence models for strongly heated internal gas flows. IASTED (Interantional Association of Scince and Technology for Development) International Conference on Modelling and Simulation, Marina del Ray, Cal., 1-3 March.

Baek, S. G., and S. O. Park,. 2004. Effect of Wall Distance Coordinate on the Prediction of Variable Property Flow with Two-Equation Turbulence Models. ICHMT International Symposium on Advances in Computational Heat Transfer, Kirken-Bergen, Norway, April. 
McEligot, D. M., J. Y. Yoo, J. S. Lee, L. E. Hochreiter, J. D. Jackson, S. O. Park, R. H. Pletcher, P. Vukoslavcevic and J. M. Wallace, 2004. Advanced computational thermal studies and their assessment for supercritical reactors. ANS 2004 Annual Meeting, Pittsburgh, 13-17 June. Trans., ANS, 90, pp. 152-153.

McEligot, D. M., and M. F. Taylor, 2004. Convective heat transfer to gas mixtures for GFR applications. ANS 2004 Annual Meeting, Pittsburgh, 13-17 June. Trans., ANS, 90, pp. 158-159.

Jackson, J. D., and J. Fewster, 2004. Some experiments on heat transfer to carbon dioxide at supercritical pressure in tubes. International Congress on Advances in Nuclear Power Plants (ICAPP '04), Pittsburgh, 13 -17 June.

He, S., P. X. Jiang and J. D. Jackson, 2004. Computational study of convective heat transfer of $\mathrm{CO}_{2}$ at supercritical pressure in a vertical mini tube. 2nd International Conference on Microchannels and Minichannels, Rochester, New York, USA, 17 -19 June.

Spall, R. E., D. M. McEligot and A. H. Richards, 2004. Numerical modeling of strongly heated internal gas flows. Presented at Fluids Engr. Conf., Charlotte, N. C., July.

Baek, S. G., and S. O. Park,. 2004. Turbulent Heat Transfer Prediction of Supercritical Flows Using Two-Equation Models. The 6th International Conference on Nuclear Thermal Hydraulics, Operations and Safety (NUTHOS-6), Nara, Japan, October .

Bae, J. H., J. Y. Yoo and H. Choi, 2004. Turbulence characteristics of thermally developing supercritical flows in a vertical annular pipe. 57th Annual Mtg., American Physical Society Division of Fluid Dynamics, Seattle, Nov. (also Bull., Amer. Phys. Soc., $\underline{49}$, [9], p. 28.)

Radulovic, I., P. V. Vukoslavcevic and J. M. Wallace, 2004. A calibration loop to test hot-wire response under supercritical conditions. 57th Annual Mtg., American Physical Society Division of Fluid Dynamics, Seattle, Nov. (also Bull., Amer. Phys. Soc., 49, [9], p. 39.)

Vukoslavcevic, P. V., I. Radulovic and J. M. Wallace, 2004. Hot- and cold-wire sensor response in supercritical $\mathrm{CO}_{2}$ flow. 57th Annual Mtg., American Physical Society Division of Fluid Dynamics, Seattle, Nov. (also Bull., Amer. Phys. Soc., 49, [9], p. 39-40.)

Bae, J. H., J. Y. Yoo and H. Choi. 2005. Influence of Strongly-Varying Properties of Supercritical Fluids on Turbulent Convective Heat Transfer. 6th KSME/JSME Thermal and Fluids Engineering Conference, Jeju, RoK. March .

Kim, J. K., H. K. Jeon, J. Y. Yoo and J. S. Lee. 2005. Experimental Study on Heat Transfer of Turbulent Supercritical CO2 Flow in a Vertical Circular Pipe. The Korean Society of Mechanical Engineering (KSME) Annual Spring Conference, Busan, Korea, May (in Korean).

McEligot, D. M., G. E. McCreery, P. D. Bayless and T. D. Marshall, 2005. Key thermal fluid phenomena in prismatic gas-cooled reactors. ANS 2005 Annual Meeting, San Diego, 5-9 June.

Wang, X., X. Xu, and R. H. Pletcher, 2005. Large eddy simulation of supercritical $\mathrm{CO}_{2}$ pipe flow with constant wall heat flux. AIAA paper 2005-4995,17th AIAA Computational Fluid Dynamics Conference, Toronto, Ontario, June 6-9.

He, S., W. S. Kim and J. D. Jackson, 2005. Modelling of turbulent heat transfer to fluid at supercritical pressure using adaptive mesh generation. 4th International Symposium on Turbulence and Shear Flow Phenomena (TSFP4), Williamsburg, Virginia, June.

Richard, A. H., R. E. Spall and D. M. McEligot, 2005. Numerical simulation of a strongly heated gas flowing upward in a vertical tube using a k-epsilon model. ASME paper HT2005-72329, presented at the Heat Transfer Conference, San Francisco, July. 
He, S., W. S. Kim, J. Fewster and J. D. Jackson, 2005. Computational simulations of experiments on convective heat transfer to carbon dioxide at a pressure just above the critical value. UK National Heat Transfer Conference, Manchester, September.

Jackson, J. D., S. He, P. X. Jiang and R. Xu, 2005. Influences of strong non-uniformity of fluid properties on turbulent heat transfer. UK National Heat Transfer Conference, Manchester, September.

Wang, J., and J. D. Jackson, 2005. Effects of thermal boundary condition on convective heat transfer under conditions of buoyancy-influenced flow in a vertical plane passage. UK National Heat Transfer Conference, Manchester, September.

Jackson, J. D., and J. Wang, 2005. An experimental study of combined radiative and convective heat transfer. UK National Heat Transfer Conference, Manchester, September.

Bae, J. H., J. Y. Yoo, H. Choi and D. M. McEligot, 2005. Influence of fluid-property variation on turbulent convective heat transfer in vertical annular channel flows. Paper 018, NuReTH-11, Avignon, France, October.

Satake, S.-i., T. Kunugi, A. M. Shehata and D. M. McEligot, 2005. DNS of forced gas flows in circular tubes at subturbulent flow. Paper 392, NuReTH-11, Avignon, France, October.

Kim, J. K., H. K. Jeon, J. Y. Yoo and J. S. Lee, 2005. Experimental study on heat transfer characteristics of turbulent supercritical flow in vertical circular/non-circular tubes. Paper 266, NuReTH-11, Avignon, France, October.

Vukoslavcevic, P., and J. M. Wallace, 2005. The dependence of Nusselt versus Reynolds number for a circular cylinder in supercritical $\mathrm{CO}_{2}$ flow. To be presented at 58th Ann. Mtg., American Physical Society Division of Fluid Dynamics, Chicago, November.

\section{Technical papers in preparation}

Baek, SG, Park, SO and Moon, CM. "Momentum and heat transfer predictions by using two-equation turbulence models for pipe flow at low Reynolds number." International Journal of Heat and Mass Transfer (submitted)

Kim JK, HK Jeon, and JS Lee. "Experimental Study on Heat Transfer of Turbulent Supercritical Carbon Dioxide Flow in Vertical Circular/Non-Circular Tubes." International Journal of Heat and Mass Transfer (submitted).

Bae, J. H., J. Y. Yoo, H. Choi and D. M. McEligot, 2006. Heat transfer and hydraulic resistance of supercriticalpressure fluid flows. In preparation for 13th Int. Heat Transfer Conf., Sydney, 13-18 August.

\section{Invited presentations}

Jackson, J. D., 2002. The naturally induced cooling experiment (NICE). European Union Concerted Action Project SCACEX. Kick-off meeting, Becker Technologies GmBH, Eschborn, Frankfurt, Germany, Feb. 5-6.

McEligot, D. M., 2002. Development of the viscous layer downstream of a roughness element in a transitioning boundary layer. Boise State Univ., Boise, Idaho, April 19.

McEligot, D. M., 2002. Recent studies with the world's largest matched-index-of-refraction flow system. Lehrstuhl für Strömungsmechanik, Uni. Erlangen, Germany, Aug.26

Univ. Limerick, Limerick, Ireland, Sept. 
Jackson, J. D., 2002. Influences of buoyancy on turbulent flow and convective heat transfer in vertical passages. Joint MU/RGU research mtg., Robert Gordon Univ., Aberdeen, Scotland, Sept.

Jackson, J. D., 2002. Consideration of the heat transfer properties of supercritical pressure water in relation to its use as a coolant in advanced nuclear reactors. 13th Pacific Basin Nuclear Conference, Shenzhen, China, Oct. 21-25.

Yoo, J. Y., 2002. Micro/bio flow diagnostic techniques in biomedical engineering, Fifth JSME-KSME Fluids Engineering Conf., Abstracts, pp. 45-49, Nagoya, Japan, Nov. 17-21.

McEligot, D. M., 2003. Advanced thermalflud physics and its assessment for advanced reactor systems. Workshop on Thermal-hydraulic Tools for Gen IV Systems, INEEL, March

Yoo, J. Y., 2003. Direct numerical simulation of heat transfer to $\mathrm{CO}_{2}$ at supercritical pressure in a vertical tube. Workshop on Supercritical Reactors, pp. 155-199, Seoul, Korea, March 23-24.

Yoo, J. Y., 2003. Recent trends in micro/bio flow visualization studies. PIV Challenge 2003, Busan, Korea, Sept. 19-20.

Buongiorno, J., and D. M. McEligot, 2003. The supercritical-pressure light-water reactor-cooled reactor (SCWR). KAIST (Korean Advanced Institute of Science and Technology), Taejon, RoK, Oct.

Yoo, J. Y., K. C. Kim, W. Kim and J. Sung, 2003. PIV application to unsteady three-dimensional flows (Keynote Lecture). 7th Asian Symposium on Visualization, Singapore, Nov. 3-7.

McEligot, D. M., 2003. Recent studies with the world's largest matched-index-of-refraction flow system. Univ. Arizona,.13 November.

Utah State Univ., 3 December.

Jackson, J. D., 2004. Impairment and enhancement of heat transfer in upward and downward flow of supercritical pressure carbon dioxide in tubes (Keynote Lecture). 3rd International Symposium on Heat Transfer Enhancement and Energy Conservation, Guangzhou, China, 2-15 January.

Jackson, J. D., 2004. The Reynolds number - A product of experiment and theory (Opening Lecture). 4th European Thermal Sciences Conference, Birmingham, March.

McEligot, D. M., 2004. Recent studies with the world's largest matched-index-of-refraction flow system.

Korea Atomic Energy Research Institute, Taejon, RoK, May 2004

Seoul National University, RoK, May 2004

University of Minnesota, September 2004

Jackson, J. D., 2004. Osborne Reynolds - His contributions to science and engineering (Guest Speaker). 7th Biennial ASME Conference, Engineering Systems Design and Analysis (ESDA 2004), Manchester, July.

Jackson, J. D., 2004. Development of a semi-empirical model of convective heat transfer to supercritical pressure fluids and consideration of its relevance to the Supercritical-Pressure Water-cooled Reactor. Foschungszentrum Karlsuhe, Germany, 23 November

Pletcher, R. H., 2005. Large-eddy simulation of flows with buoyancy, rotation and heat transfer. Wayne State Univ, 11 February Korea Advanced Institute of Science and Technology, Taejon, Korea, 19 May

Korea Aerospace Research Institute, Taejon, Korea, 19 May

Jackson, J. D., 2005. Osborne Reynolds's pioneering contribution to turbulent convective heat transfer. Heat Transfer Society, London, February. 
Jackson, J. D., 2005. Impairment and enhancement of heat transfer in upward and downward flow of supercritical pressure carbon dioxide in tubes. Brunel Univ., London, 3 March

Pletcher, R. H., 2005. Application of large eddy simulation to problems in supercritical reactors. Int. Workshop on Supercritical Water-Cooled Reactors, Seoul National Univ., 16 May

Jackson, J. D., 2005. Influences on turbulence heat transfer of strong non-uniformity of fluid properties near the critical point. Int. Workshop on Supercritical Water-Cooled Reactors, Seoul National Univ., 16 May

\section{Other publications}

Kang, H. S., and J. Y. Yoo, 2002. Turbulence characteristics of the three-dimensional boundary layer on a rotating disk with jet impingement. Experiments in Fluids, $\underline{33}$, pp. 270-280.

Nam, Y. S., H. G. Choi and J. Y. Yoo, 2002. AILU preconditioning for the finite element formulation of the incompressible Navier-Stokes equations. Computer Methods in Applied Mechanics and Engineering, 191, pp. 43234339 .

Park, N., and J. Y. Yoo, 2002. On constructing an explicit algebraic stress, model without wall-damping function. KSME Int. J., 16 (11), pp. 1522-1539.

Sung, J., and J. Y. Yoo, 2002. Vortex lock-on and spatio-temporal evolutions of streamwise vortices. $J$. Visualization, $\underline{5}$ (4), p. 315.

Kim, J. H., J. Y. Yoo and S. H. Kang, 2002. Dilatation and vortex stretching effects on turbulence in onedimensional/axisymmetric flows. AIAA J., $\underline{40}$ (12), pp. 2476-2482.

Jin, S., S. Kim, H. Choi, J. Y. Yoo and S. Kim, 2002. Effect of two-fequency forcing on flow behind a backwardfacing step. Transactions of the Korean Society of Mechanical Engineers B, 26 (3), pp. 423-431.

Kim, J. K., J. Sung, J. K. Chang, J. Y. Yoo and B. G. Min, 2002. PIV measurements of flow downstream of polyurethane heart valve, prosthesis for artificial heart: pulsatile flow experiment. Transactions of the Korean Society of Mechanical Engineers B, 26 (5), pp. 629-639.

Park, T. H., H. G. Choi, J. Y. Yoo and S. J. Kim, 2002. Streamline-upwind numerical simulation of twodimensional confined impinging slot jets. Transactions of the Korean Society of Mechanical Engineers B, 26 (12), pp. 1663-1673.

Namkoong, K., H. Choi and J. Y. Yoo, 2002. Computation of dynamic, fluid-structure interaction in a 2dimensional laminar channel flow divided by a plate. Transactions of the Korean Society of Mechanical Engineers $B, \underline{26}$ (12), pp. 1738-1746.

Choi, H. G., P. Huang, D. Joseph and J. Y. Yoo, 2002. Wall effect on the settling motion of a slender object in a two-dimensional channel. Bulletin of the American Physical Society, 47 (1), p. 535, Indianapolis, Indiana, March $18-22$.

Kim, S., H. Choi and J. Y. Yoo, 2002. Active control of a backward-facing step flow with double-frequency forcing. Advances in Turbulence Research-2002, pp. 33-49, Seoul National Univ., Seoul, Korea, April 27.

Yoo, J. Y., 2002. Micro/nanohydrodynamics for biomedical applications. J. Visualization Society of Japan, 22 (1), pp. 11-14 (Keynote Lecture, Proceeding of the 30th Symposium on Visualization, Kogakuin Univ., Tokyo, Japan, July 22-24). 
Min, T., H. Choi, J. Y. Yoo, 2002. Maximum drag reduction in a turbulent channel flow by polymer additives. Bulletin of the American Physical Society, Program of the 55th Annual Mtg. of the Division of Fluid Dynamics, 47 (10), p. 53, Dallas, Texas, Nov. 24-26.

Park, N., J. Y. Yoo and H. Choi, 2002. Revisiting numerical errors in large eddy simulation of turbulence. Bulletin of the American Physical Society, Program of the 55th Annual Mtg. of the Division of Fluid Dynamics, 47 (10), p. 141, Dallas, Texas, Nov. 24-26.

Lee, J. S., and I. S. Jung, 2002. Effect of bulk flow pulsations on film cooling with compound angle holes. Int. J. Heat Mass Transfer, $\underline{45}$ (1), pp. 113-123.

Lee, H. W., J. J. Park, J. S. Lee, 2002. Flow visualization and film cooling effectiveness measurements around shaped holes with compound angle orientations. Int. J. Heat Mass Transfer, $\underline{45}$ (1), pp.145-156.

Jung, I. S., J. S. Lee and P. M. Ligrani, 2002. Effects of bulk flow pulsations on film cooling with compound angle holes: heat transfer coefficient ratio and heat flux ratio. ASME J. Turbomachinery, 124. (1), pp. 142-151.

Kim, S. K., W. I. Lee and J. S. Lee, 2002. Solving a nonlinear inverse convection problem using the sequential gradient method. KSME Int. J., 16 (5), pp. 710-719.

Lee, J. S., H. G. Jung and S. B. Kang, 2002. Effect of embedded vortices on film cooling performance on a flat plate. Experimental Thermal and Fluid Science, 26, pp. 197-204.

Park, J. S., M. S.Kim, J. S. Lee and W. I. Lee, 2002. A semi-implicit method for the analysis of two-dimensional fluid flow with moving free surfaces. KSME Int. J., 16 (5), pp. 720-731.

Lee, S. W., J. S. Lee and K. Kuk, 2002. Effect of concave wall curvature on three-dimensional behavior of film cooling jet. KSME Int. J., 16 (9), pp.1121-1136.

Han, B., D. K. Sohn and J. S. Lee, 2002. Flow and heat transfer measurements of film injectant from a row of holes with compound angle orientations. KSME Int. J., 16 (9), pp.1137-1146.

Lee, J., S. Park, O. Kwon, Y. K. Choi and J. S. Lee, 2002. Characteristics of liquid thin film using molecular dynamics simulation. KSME Int. J., 16 (11), pp.1477-1484.

Chung, W. S., Y. C. Sa and J. S. Lee, 2002. An experimental study on dryout pattern of two-phase flow in helically coiled tubes. KSME Int. J., 16 (11), pp.1540-1549.

Avancha, R. V. R., and R. H. Pletcher, 2002. Large eddy simulation of the turbulent flow past a backward-facing step with heat transfer and property variations. Int. J. Heat Fluid Flow, 23, pp. 601-614.

Becker, S., C. M. Stoots, K. G. Condie, F. Durst and D. M. McEligot, 2002. LDA-measurements of transitional flows induced by a square rib. J. Fluids Engineering, 124, pp. 108-117.

Lee, J. S., and R. H. Pletcher, 2002. Large eddy simulation of the effects of rotation on heat transfer in a ribbed channel. Heat Transfer 2002, Proc., 12th Int. Heat Transfer Conf., Grenoble.

McCreery, G. E., K. G. Condie, R. L. Clarksean and D. M. McEligot, 2002. Convective processes in spent nuclear fuel canisters. Heat Transfer 2002, Proc., 12th Int. Heat Transfer Conf., Paper IHTC-1267, pp. 663-668, Grenoble, August.

Jackson, J. D., P. An and M. Ahmadinejad, 2002. Effects of non-condensible gas on the condensation of steam. Heat Transfer 2002, Proc., 12th Int. Heat Transfer Conf., Grenoble. 
Simoens, S., M. Ayrault and J. M. Wallace, 2002. Scalar transport in the cavity between two square two-dimensional obstacles at the wall of a turbulent boundary layer. 55th Annual Meeting, American Physical Society Division of Fluid Dynamics, Dallas, Nov. (also Bull., Amer. Phys. Soc., 47 (10), pp. 165-166.

He, S., Z. Xu and J. D. Jackson, 2003. An experimental investigation of buoyancy-opposed wall jet flow. Int. J. Heat Fluid Flow, 23, pp. 487-496.

Park, T. H., H. G. Choi, J. Y. Yoo and S. J. Kim, 2003. Streamline upwind numerical simulation of twodimensional confined impinging slot jets. Int, J. of Heat and Mass Transfer, 46, pp. 251-262.

Sung, J., and J. Y. Yoo, 2003. Near-wake vortex motions behind a circular cylinder at low Reynolds number. $J$. Fluids and Structures, 17, pp. 261-274.

Min, T., J. Y. Yoo, H. Choi and D. D. Joseph, 2003. Drag reduction by polymer additives in a turbulent channel flow. J. Fluid Mechanics, 486, pp. 213-238.

Lee, Y., J. K. Chang, S. H. Lee, J. U. Jang, K. B. Lee, H. W. Lee, K. Sun. H. M. Kim, J. Kim and J. Y. Yoo, 2003. Development of magnetically suspended vaneless centrifugal blood pump for cardiopulmonary bypass. $J$. Mechanics in Medicine and Biology, $\underline{3}$ (2), pp. 187-196.

Min, T., H. Choi and J. Y. Yoo, 2003. Maximum drag reduction in a turbulent channel flow by polymer additives. J. Fluid Mech., $\underline{492}$, pp. 91-100.

Kim, W., J. Sung and J. Y. Yoo, 2003. High-definition PIV analysis on vortex shedding in the cylinder wake. J. Visualization, 7 (No. 1), pp. 17-24.

Kim, J. K., C. Chung, J. K. Chang and J. Y. Yoo, 2003. Analysis of fully developed multilayer flow in microchannel with a rectangular cross section. Transactions of the Korean Society of Mechanical Engineers B, 27 (5), pp. 644-654.

Kang, S., H. Choi and J. Y. Yoo, 2003. Parallel preconditioner for the domain decomposition method of the discretized navier-stokes equation. Transactions of the Korean Society of Mechanical Engineers B, 27 (6), pp. 753765.

Park, N., J. Y. Yoo and H. Choi, 2003. On the suitability of centered and upwind-biased compact difference schemes for large eddy simulation (_) - numerical test. Transactions of the Korean Society of Mechanical Engineers B, 27 (7), pp. 973-983.

Park, N., J. Y. Yoo and H. Choi, 2003. On the suitability of centered and upwind-biased compact difference schemes for large eddy simulations $\left(\_\right)$- static error analysis. Transactions of the Korean Society of Mechanical Engineers B, 27 (7), pp. 984-994.

Park, N., J. Y. Yoo and H. Choi, 2003. On the suitability of centered and upwind-biased compact difference schemes for large eddy simulations $\left(\_\right)$- dynamic error analysis. Transactions of the Korean Society of Mechanical Engineers B, 27 (7) pp. 995-1006.

Kim, S., H. Choi and J. Y. Yoo, 2003. Double frequency forcing of the laminar separated flow over a backwardfacing step. Transactions of the Korean Society of Mechanical Engineers B, 27 (8), pp. 1023-1032.

Kim, B., S. Kang, H. Choi and J. Y. Yoo, 2003. Numerical study on the drag of a car model under road condition. Transactions of the Korean Society of Mechanical Engineers B, 27 (8), pp. 1082-1090.

Kim, J. K., H. Bang, S. Chung, A. D. Chin-Yu, C. Chung, H. S. Jo, J. Y. Yoo and J. K. Chang, 2003. Single-cell manipulation and fluorescence detection in benchtop flow cytometry system with disposable plastic microfluidic chip. In Microfluidics, BioMEMS, and Medical Microsystems (Ed: H. Becker, P. Woias) (Also Proc., SPIE, 4982 (2003), pp. 8-20, San Jose, California, Jan. 27-29, 2003). 
Kim, S., and J. Y. Yoo, 2003. Fluid motion near the meniscus in the hydrophilic and hydrophobic micro-capillaries, APS DFD 56th Annual Meeting, East Rutherford, New Jersey, Nov. 23-25. (Also Bull. APS, 48, No. 10, p. 223).

Kim, S. K., J. S. Lee and W. I. Lee, 2003. A solution method for a nonlinear three-dimensional inverse heat conduction problem using the sequential gradient method combined with cubic-spline function specification. Numerical Heat Transfer: Part B, 43 (1), pp. 43-61.

Ahn, J., I. S. Jung and J. S. Lee, 2003. Film cooling from two rows of holes with opposite orientation angles: Injectant behavior and adiabatic film cooling effectiveness. Int. J. Heat and Fluid Flow, 24 (1), pp.91-99.

Chung, J. D., B. Y. Park and J. S. Lee, 2003. The combined effects of angle of attack and louver angle of a winglet pair on heat transfer enhancement. J. Enhanced Heat Transfer, 10 (1), pp. 31-44.

McEligot, D. M., 2003. Low-Reynolds-number turbulent flow? Amer. Physical Soc. Fluid Dynamics Mtg, East Rutherford, Nov. (Also Bull. APS, $\underline{48}$, No. 10, p. 50).

Walsh, E. J., M. R. D. Davies and D. M. McEligot, 2004. On the use of entropy to predict boundary layer stability. J. Entropy, $\underline{6}$, pp. 375-387.

McCreery, G. E., and D. M. McEligot, 2004. Discussion of "Transition to meandering rivulet flow in vertical parallel-plate channels." J. Fluids Engr., 126, pp. 498-499.

Ergun, S., J. G. Williams, L. E. Hochreiter, H. Wiersema, M. Stootman and M. Stempniewicz, 2004. Validation of COBRA-TF critical heat flux predictions for a small hydraulic diameter geometry under natural boiling conditions. ANS 2004 Annual Meeting, Pittsburgh, 13-17 June. Trans., ANS, 90, pp. 575-576.

Lee, J. S., N. Meng, R.H. Pletcher, Y. Liu, "Numerical Study of the Effects of Rotation on Heat Transfer in Channels with and without Ribs," International Journal of Heat and Mass Transfer, Vol. 47, pp. 4673-4684, 2004.

Miller, D. J., L. E. Hochreiter, F.-B. Chung, B. R. Lowery, T. F. Lin, G. S. Rhee, S. M. Bajorek and J. E. Kelly, 2004. Two-phase level swell, interfacial drag experiments in the Pennsylvania State University/US Nuclear Regulatory Commission Rod Bundle Heat Transfer Facility ANS 2004 Annual Meeting, Pittsburgh, 13-17 June. Trans., ANS, 90, pp. 492-493].

McEligot, D. M., and M. F. Taylor, 2004. Convective heat transfer to gas mixtures for GFR applications. ANS 2004 Annual Meeting, Pittsburgh, 13-17 June. Trans., ANS, 90, pp. 158-159.

Lee, J. S., X. Zu, and R. H. Pletcher, "Large Eddy Simulation of the Effects of Inner Wall Rotation on Heat Transfer in Annular Turbulent Flow," Numerical Heat Transfer, Part A, Vol. 46, pp. 323-341, 2004.

McCreery, G. E., D. M. McEligot and P. Meakin, 2004. Transitions in rivulet flow. To be presented at Amer. Physical Soc. Fluid Dynamics meeting, Seattle, November. Bull. APS, 49, No. 9, pp. 33-34.

C.Y. Zhao, T.J. Lu, H.P. Hodson and J.D. Jackson, 2004. 'The Temperature Dependence of Effective Thermal Conductivity of Open-Celled Steel Alloy Foams', Materials Science and Engineering: A, 367, pp. 123-131.

Qin, Z., and R. H. Pletcher, 2004. Large eddy simulation of turbulent heat transfer in a rotating square duct. 57th Annual Mtg., American Physical Society Division of Fluid Dynamics, Seattle, Nov. (also Bull., Amer. Phys. Soc., 49, [9], p. 217.)

Lee, J. S., X. Xu, and R. H. Pletcher, "Effects of Wall Rotation on Heat Transfer to Annular Turbulent Flow: Outer Wall Rotating," Journal of Heat Transfer, Vol. 127, pp. 830-838, 2005.

McEligot, D. M., and H. Eckelmann, 2005. Laterally converging duct flows: Part 3. Mean turbulence structure in the viscous layer. J. Fluid Mech., in press. 


\section{References cited}

Abe, K., T. Kondoh and Y. Nagano, 1996. A two-equation heat transfer model reflecting second-moment closures for wall and free turbulent flows. Int. J. Heat Fluid Flow, 17, pp. 228-237.

Abid, R., C. L. Rumsey and T. B. Gatski, 1995. Prediction of nonequilibrium turbulent flows with explicit algebraic models. AIAA Journal, $\underline{33}$ (11), pp. 2026-2031.

Ackerman, J. W., 1970. Pseudoboiling heat transfer to supercritical pressure water in smooth and ribbed tubes. $J$. Heat Transfer, $\underline{92}$, pp. 490-498.

Adeyinka, O. B., and G. F. Naterer, 2005. Experimental uncertainty of measured entropy production with pulsed laser PIV and planar laser induced fluorescence. Int J. Heat Mass Transfer, 48, pp. 1450-1461.

Avancha, R. V. R., and R. H. Pletcher, 2002. Large eddy simulation of the turbulent flow past a backward-facing step with heat transfer and property variations. Int. J. Heat Fluid Flow, 23, pp. 601-614.

Bae, J. H., J. Y. Yoo and H. Choi, 2003. Direct numerical simulation of strongly-heated internal gas flows with large variations of fluid properties. 56th Annual Mtg., American Physical Society Division of Fluid Dynamics, East Rutherford, N. J., Nov. (also Bull., Amer. Phys. Soc., 4, [10], p. 22.)

Bae, J. H., J. Y. Yoo and H. Choi. 2005. Direct numerical simulation of turbulent supercritical flows with heat transfer. Physics of Fluids, in press.

Bae, J. H., J. Y. Yoo, H. Choi and D. M. McEligot, 2005. Influence of fluid-property variation on turbulent convective heat transfer in vertical annular channel flows. Paper 018, NuReTH-11, Avignon, France, October.

Baek, S. G., and S. O. Park, 2003. Prediction of strongly-heated gas flows in a vertical tube using explicit algebraic stress/heat-flux models. Paper E00212, NuReTH-10, Seoul, Korea, October.

Baek, S. G., and S. O. Park, 2004. Effect of Wall Distance Coordinate on the Prediction of Variable Property Flow with Two-Equation Turbulence Models. ICHMT International Symposium on Advances in Computational Heat Transfer, Kirken-Bergen, Norway, April.

Balaras, E., 2004. Modeling complex boundaries using an external force filed on fixed Cartesian grids in large-eddy simulations. Computers and Fluids, $\underline{33}$, pp. 375-404.

Battista, E., and H. C. Perkins,1970. Turbulent heat and momentum transfer in a square duct with moderate property variations. Int. J. Heat Mass Transfer, 13, pp. 1063-1065.

Becker S., C. M. Stoots, K. G. Condie, F. Durst and D. M. McEligot, 2002. LDA-measurements of transitional flows induced by a square rib. J. Fluids Engr., 124, pp. 108-117.

Bellmore, C. P., and R. L. Reid, 1983. Numerical prediction of wall temperatures for near-critical para-hydrogen in turbulent upflow inside vertical tubes, J. Heat Transfer, 105: pp. 536-541.

Berner, C., F. Durst and D. M. McEligot, 1984. Flow over baffles. J. Heat Transfer, 106, pp. 743-749.

Blackwelder, R.F., 1981. Hot-wire and hot-film anemometers. Methods of Experimental Physics: Fluid Dynamics, New York: Academic Press.

Campbell, D. A., and H. C. Perkins, 1970. Variable property turbulent heat and momentum transfer for air in a vertical rounded corner triangular duct. Int. J. Heat Mass Transfer, 11, pp. 1003-1012. 
Carlson, L. W. and T. F. Irvine, Jr., 1961. Fully developed pressure drop in triangular shaped ducts. J. Heat Transfer, $\underline{83}$, pp. 441-444.

Celata, G. P., F. D'Annible, A. Chiaradia and M. Cumo, 1998. Upflow turbulent mixed convection heat flow in vertical pipes. Int. J. Heat Mass Transfer, 141, pp. 4037-4054.

Cheng X., and T. Schulenberg, 2001. Heat transfer at the supercritical pressures - Literature review and application to an HPLWR,. Technical report, Institut für Kern und Energietechnik Programm, Nukleare Sicherheitsforschung, Forschungszentrum Karslruhe.

Cheng, X., T. Schulenberg, D. Bitterman and P. Rau, 2003. Design analysis of core assemblies for supercritical steam conditions. Nuc. Engr. Design, 223, pp. 279-294.

Chien, K. Y., 1982. Predictions of channel and boundary-layer flows with a low-Reynolds-number turbulence model, AIAA J., 20 (1), pp. 33-38.

Chung, S. Y., G. H. Rhee and H. J. Sung, 2002. Direct numerical simulation of turbulent concentric annular pipe flow, Part 1: Flow field. Int. J. Heat and Fluid Flow, 23, pp. 426-440.

Corino, E. R., and R. S. Brodkey, 1969. A visual observation of the wall region in turbulent flow. J. Fluid Mech., 37, pp. 1-30.

Cotton, M. A., J. O. Ismael and P. J. Kirwin, 2001. Computations of post-trip reactor core thermal hydraulics using a strain parameter turbulence model. Nucl. Eng. Design, 208, pp. 51-66.

Cui, M. M., and R. J. Adrian, 1997. Refractive index matching and marking methods for highly concentrated solidliquid flows. Exp. Fluids, 22, pp. 261-264.

Dailey, L. D., N. Meng and R. H. Pletcher, 2003. Large eddy simulation of constant heat flux turbulent channel flow with property variations: Quasi-developed model and mean flow results. J. Heat Transfer, $\underline{125}$, pp. 27-38.

Dang, K.D. \& Morchoisne, Y, 1989. Numerical methods for direct simulation of turbulent shear flows. Von Karman Institute Lecture Series 1989-03.

Davis, C. B., 2003. Personal communication. Idaho National Engineering and Environmental Laboratory, 25 September.

Dittus, F. W., and L. M. K. Boelter, 1930. Heat transfer in automobile radiators of the tubular type. Univ. Calif. Publ. in Engr., 2, pp. 443-461.

Durst, F., J. Jovanovic and J. Sender, 1993. Detailed measurements of the near wall region of turbulent pipe flows. Proc., 9th Symp. Turb. Shear Flows, paper 2-2, Kyoto.

Durst, F., T. Keck and R. Kleine, 1979. Turbulence quantities and Reynolds stress in pipe flow of polymer solutions measured by two-channel laser-Doppler anememetry. Proc., 6th Symp. Turbulence, Rolla, Missouri.

Eggels, J. G. M., F. Unger, M. H. Weiss, J. Westerweel, R. J. Adrian, R. Friedrich and F. T. M. Nieuwstadt, 1994. Fully developed turbulent pipe flow: A comparison between direct numerical simulation and experiment. J. Fluid Mech., 268, pp. 175-209

Fadlun, F. A., R. Verzicco, P. Orlandi and J. Mohd-Yusof, 2000. Combined immersed-boundary finite-difference methods for three-dimensional complex flow simulations. J. Computational Physics, 161, pp. 35-60.

Gatski, T. B., and C. G. Speziale, 1993. On explicit algebraic stress models for complex turbulent flows. J. Fluid Mech., 254, pp. 59-78. 
Habib, M. A., F. Durst and D. M. McEligot, 1984. Streamwise-periodic flow around baffles. Proc., 2nd Int. Symp. Appl. Laser Anemometry to Fluid Mechanics, Lisbon, July.

Hall, W. B., 1971. Heat transfer near the critical point. Adv. Heat Transfer, $\underline{7}$, New York: Academic Press.

Hanjalic, K., 1999. Second-moment turbulence closures for CFD: Needs and prospects. Int. J. Comp. Fluid Dyn., $\underline{12}$, pp. 67-97.

Hanjalic, K., 2002. One-point closure models for buoyancy-driven turbulent flows. Ann. Rev. Fluid Mech., $\underline{34}$, pp. 321-347.

Hartnett, J. P., J. C. Y. Koh and S. T. McComas, 1962. A comparison of predicted and measured friction factors for turbulent flow through rectangular ducts. J. Heat Transfer, $\underline{84}$, pp. 82-88.

He, S., W. S. Kim, P. X. Jiang and J. D. Jackson, 2003. Simulation of mixed convection heat transfer to carbon dioxide at supercritical pressure. 8th UK Nat. Heat Transfer Conf., Oxford, 9-10 September.

He, S., W. S. Kim, P. X. Jiang and J. D. Jackson, 2004. Simulation of mixed convection heat transfer to carbon dioxide at supercritical pressure. Int. J. Mech. Eng. Sci., Proc. I. Mech. E., 218, Part C, Special Issue, pp. 12811296.

Hess, H. L., and H. R. Kunz, 1965. A study of forced convection heat transfer to supercritical hydrogen. J. Heat Transfer, $\underline{87}(1)$, pp. 41-48.

Hess, W. G., A. F. Deardorff and D. M. McEligot, 1971. Radiation thermocouple conduction error. Technical memorandum, Energy, Mass and Momentum Tranfer Laboratory, Aero. Mech. Engng. Dept., U. Arizona. 7 June.

Holman, J. P., 2001. Experimental methods for engineers, 7th Ed. New York: McGraw-Hill

Hsu, Y.-Y., and J. M. Smith, 1961. The effect of density variation on heat transfer in the critical region. J. Heat Transfer, $\underline{83}$ (2), pp. 176-182.

Jackson, J. D., 2001. Some striking features of heat transfer with fluids at pressures and temperatures near the critical point (Keynote paper). Int. Conf. Energy Conv. and Appl. (ICECA 2001), Wuhan, China.

Jackson, J. D., 2002. Consideration of the heat transfer properties of supercritical pressure water in connection with the cooling of advanced nuclear reactor. Proc.,13th Pacific Basin Nuclear Conference, Shenzhen City, China, 21-25 October, pp. 21-25.

Jackson, J. D., 2005. Private communication, University of Manchester.

Jackson, J. D., M. A. Cotton and B. P. Axcell, 1989. Studies of mixed convection in vertical tubes. Int. J. Heat Fluid Flow, 10, pp. 2-15.

Jackson, J. D., and W. B. Hall, 1979a. Forced convection heat transfer to fluids at supercritical pressure. Turbulent forced convection in channel and bundles, (Ed.: S. Kakac and D. B. Spalding,), vol. 2. Washington: Hemisphere Publishing Corporation, pp. 563-611.

Jackson, J. D., and W. B. Hall, 1979b. Influence of buoyancy on heat transfer to fluids flowing in vertical tubes under turbulent conditions. Turbulent forced convection in channel and bundles, (Ed.: S. Kakac and D. B. Spalding,), vol. 2. Washington: Hemisphere Publishing Corporation, pp. 613-640.

Jevremovic, T., Y. Oka and S.-i. Koshizuka, 1996. $\mathrm{UO}_{2}$ core design of a direct-cycle fast converter reactor cooled by supercritical water. Nuc. Tech., 114 , pp. 273-284. 
Jones, W. P., and B. E. Launder, 1973. The calculation of low-Reynolds-number phenomena with a two-equation model of turbulence. Int. J. Heat and Mass Transfer, 16, pp. 1193-1130.

Jorgensen F. (1971) Directional sensitivity of wire and fibre film probes. DISA Inf. 11:31-37.

Kays, W. M., and M. E. Crawford, 1993. Convective heat and mass transfer, 3rd ed. New York: McGraw-Hill.

Kenjeres, S., and K. Hanjalic, 2000. Convective rolls and heat transfer in finite-length Rayleigh-Benard convection: A two-dimensional numerical study. Phys. Rev., E 62, pp. 7987-7998.

Kenjeres, S., and K. Hanjalic, 2002. Convective rolls and heat transfer in finite-length Rayleigh-Benard convection: A two-dimensional numerical study. Physics of Fluids, $\underline{62}$ (6), pp. 7987-7998.

Kim, J. K., H. K. Jeon, J. Y. Yoo and J. S. Lee, 2005. Experimental study on heat transfer characteristics of turbulent supercritical flow in vertical circular/non-circular tubes. Paper 266, NuReTH-11, Avignon, France, October.

Kirillov, P. L., 2000. Heat and mass transfer at supercritical parameters (The short review of researchers in Russia. Theory and experiments). Proc., First Int. Sym. on Supercritical Water-Cooled Reactor Design and Technology (SCR-2000), Tokyo, Japan, 6-8 November, Paper 105.

Kline, S. J., W. C. Reynolds, F. A. Schraub and P. W. Rundstadler, 1967. The structure of turbulent boundary layers. J. Fluid Mechanics, $\underline{30}$, pp. 741-773.

Koshizuka, S., N. Takano and Y. Oka, 1995. Numerical analysis of deterioration phenomena in heat transfer to supercritical water. Int. J. Heat Mass Transfer, $\underline{38}$ (16), pp. 3077-3084.

Kraemer, W., G. Proebstle, W. Uebelhack, T. Keheley, K. Tsuda and S. Kato, 1995. The ULTRAFLOW spacer -an advanced feature of ATRIUM fuel assemblies for boiling water reactors. Nuc. Engr. Design, 154, pp. 17-21.

Kramers, H., 1946. Heat transfer from spheres to flowing media. Physica, $\underline{12}$, pp. 61-80.

Kransnoshchekov, E. A., and V. S. Protopopov, 1966. Experimental study of heat exchange in carbon dioxide in the supercritical range at high temperature drops. High Temperatures, 4 , pp. 375-382.

Kurganov, V. A., and V. B. Ankudinov, 1986. Experimental study of velocity and temperature fields in an ascending flow of carbon dioxide at supercritical pressure in a heated vertical pipe. High Temperature, 24(6), pp. 811-818.

Kurganov, V. A., and A. G. Kaptilnyi, 1993. Flow structure and turbulent transport of a supercritical pressure fluid in a vertical heated tube under the conditions of mixed convection. Experimental data. Int. J. Heat Mass Transfer, $\underline{36}$ (12), pp. 3383-3392.

Launder, B. E., and B. I. Sharma, 1974. Application of the energy dissipation model of turbulence to the calculation of flow near a spinning disc. Lett. Heat Transfer, 1 , pp. 131-138.

Launder, B. E., and D. B. Spalding, 1972. Mathematical models of turbulence. New York: Academic Press.

Lee, J. S. , and R. H. Pletcher, 2001. Large eddy simulation of variable property turbulent flow in a vertical channel with buoyancy effects and heat transfer. ASME paper NHTC01-1555, 35th National Heat Transfer Conference.

Lee, J. S., and R. H. Pletcher, 2001. Large eddy simulation of variable property turbulent flow in vertical channel with buoyancy effects and heat transfer. 35th National Heat Transfer Conf., ASME paper NHTC 2001-20246, Anaheim, June. 
Lee, J. S., X. Xu, and R. H. Pletcher, 2004. Large eddy simulation of heated vertical annular pipe flow in fully developed turbulent mixed convection, International Journal of Heat and Mass Transfer, 47, pp. 437-446.

Lee, S. H., and J. R. Howell, 1996a. Laminar forced convection at zero gravity to water near the critical region. $J$. Thermophysics Heat Transfer, 10, pp. 504-510.

Lee, S. H., and J. R. Howell, 1996b. Gravitational effects on laminar convection to near-critical water in a vertical tube. J. Thermophysics Heat Transfer, 10, pp. 627-632.

Lee, S. H., and J. R. Howell, 1998. Turbulent developing convective heat transfer in a tube for fluids near the critical point. Int. J. Heat Mass Transfer, 41 (10): 1205-1218.

Li, L. J., C. X. Lin and M. A. Ebadian, 1999. Turbulent heat transfer to near-critical water in a heated curved pipe under the conditions of mixed convection. Int. J. Heat Mass Transfer, $\underline{42}$ (16), pp. 3147-3158.

Liuboja, M., and W. Rodi, 1981. Prediction of horizontal and vertical turbulent buoyant wall jets, J. Heat Transfer, $\underline{103}$, pp. 343-349.

MacDonald, P. E., J. Buongiorno, C. B. Davis and K. D. Weaver, 2002. Feasibility study of supercritical light water cooled fast reactors for actinide burning and electric power production. Tech. report INEEL/EXT-02-00925, June.

McCreery, G. E., K. G. Condie, R. L. Clarksean and D. M. McEligot, 2002. Convective processes in spent nuclear fuel canisters. Heat Transfer 2002, pp. 663-668 (Proc., 12th Int. Heat Transfer Conf., Grenoble).

McCreery, G. E., R. J. Pink, K. G. Condie and D. M. McEligot, 2003. Fluid dynamics of ribbed annuli. Paper J00203, NuReTH-10, Seoul, Oct.

McEligot, D. M, 1986. Convective heat transfer in internal gas flows with temperature-dependent properties. Adv. Transport Processes, 4, pp 113-200.

McEligot, D. M. and Jackson, J. D., 2004. Deterioration criteria for convective heat transfer in gas flow through non-circular ducts. Nuclear. Eng. Design. 232, pp. 327-333.

McIlroy, H. M., and R. J. Pink, 2005. KNERI model location study results. Informal technical memorandum, Idaho National Laboratory, 1 August.

McKinden, M.O., S. A. Klein, E. W. Lemmon and A. P. Peskin, 1998. REFPROP. NIST Standard Reference Database 23, Ver. 6.0.

Menter, F., and T. Esch, 2001. Advanced turbulence modelling in CFX. CFX Update, 20 Spring, pp. 4-5.

Mikielewicz, D. P., 1994. Comparative studies of turbulence models under conditions of mixed convection with variable properties in heated vertical tubes. Ph.D. thesis, Univ. Manchester.

Mikielewicz, D. P., A. M. Shehata, J. D. Jackson and D. M. McEligot, 2002. Temperature, velocity and mean turbulence structure in strongly-heated internal gas flows. Comparison of numerical predictions with data. Int. J. Heat Mass Transfer, 45, pp. 4333-4352.

Morinishi Y., T.S. Lund, O. V. Vasilyev and P. Moin, 1998. Fully conservative higher order finite difference schemes for incompressible flow. J. Computational Physics, 143, pp. 90-124.

NIST Chemistry Web Book, http://webbook.nist.gov/chemistry/

Oka, Y., 2000. Review of high temperature water and steam cooled reactor concepts. SCR-2000, Tokyo, November. 
Orr, B., E. Thomson and R. S. Budwig, 1997. Drakeol 5 thermophysical property measurements. Mech. Engng. Dept., U. idaho.

Parker, J., and P. Merati, 1996. An investigation of turbulent Taylor-Couette flow using laser Doppler velocimetry in a refractive index matched facility. J. Fluids Engr., 118, pp. 810-818.

Petukhov, B. S., 1970. Heat transfer and friction in turbulent pipe flow with variable physical properties. Adv. Heat Transfer, $\underline{6}$, New York: Academic Press.

Petukhov, B. S., and Polyakov, A. F., 1988. Heat transfer in turbulent mixed convection. Washington: Hemisphere Publishing Corporation.

Pierce, C.D., 2001. Progress-variable approach for large-eddy simulation of turbulent combustion. Ph. D. Thesis, Stanford University

Pletcher, R. H., 1999. Personal communication, Iowa State Univ.

Popov, V. N., 1983. The effects of free convection on turbulent transport for a liquid flowing in a vertical channel. Teplofizika Vysokikh Temperatur, 21 (3), pp. 515-521.

Popov, V. N., and V. M. Belyaev, 1975. Heat transfer with transitional and turbulent flow conditions of a liquid in a round tube at small Reynolds numbers. Teplofizika Vysokikh Temperatur, 13 (2), pp. 370-378.

PROPATH Group, 1999. A PROgram PAckage for Thermophysical properties of fluids (PROPATH), ver. 11.1. The International Association for the Properties of Water and Stream (IAPWS), http://www.iapws.org.

PROPATH Group, 2001. PROPATH : A Program Package for Thermophysical Properties, Ver. 12.1.

Rehme, K. , 1992. Experimental investigation of the redistribution of turbulent flow in a rod bundle downstream of a spacer grid. NuReTH-5, Salt Lake City, Vol. I, pp. 235-241.

Satake, S.-i., T. Kunugi, A. M. Shehata and D. M. McEligot, 2000. Direct numerical simulation on laminarization of turbulent forced gas flows in circular tubes with strong heating. Int. J. Heat Fluid Flow, 21, pp. 526-534.

Satake, S.-i., T. Kunugi, A. M. Shehata and D. M. McEligot, 2002. DNS of forced gas flows in circular tubes at various heating rates. 12th Int. Heat Transfer Conf., Presentation OFS 12, Grenoble, Aug.

Satake, S.-i., T. Kunugi, A. M. Shehata and D. M. McEligot, 2005. DNS of forced gas flows in circular tubes at subturbulent flow. Paper 392, NuReTH-11, Avignon, France, October.

Schneider, P. J., 1955. Conduction Heat Transfer. Reading: Addison-Wesley Publishing Company, Inc., pp. 173181.

Shehata, A. M., and D. M. McEligot, 1998. Mean turbulence structure in the viscous layer of strongly-heated internal gas flows. Measurements. Int. J. Heat Mass Transfer, 41, pp. 4297-4313.

Shiralkar, B. S., and P. Griffith, 1969. Deterioration in heat transfer to fluids at supercritical pressure and high heat fluxes. J. Heat Transfer, 90 (1), pp. 27-36.

Shiralkar, B., and P. Griffith, 1970. The effect of swirl, inlet conditions, flow direction and tube diameter on the heat transfer to fluids at supercritical pressure. J. Heat Transfer, $\underline{92}$, pp. 465-474.

Smith, B.L., 2005. Particle image velocimetry measurements of turbulent flow through a rod bundle. Technical report, Mech. Engng. Dept. Utah State Univ., 18 October. 
So, R. M. C., P. Vimala, L. H. Jin, C. Y. Zhao and T. B. Gatski, 2002. Accounting for buoyancy effects in the explicit algebraic stress model: Homogeneous turbulent shear flows. Theo. Comp. Fluid Dyn., 15, pp. 283-302.

Stoots, C. M., S. Becker, K. G. Condie, F. Durst and D. M. McEligot, 2001. A large-scale matched-index-ofrefraction flow facility for LDA studies of complex geometries. Exp. Fluids, 30, pp. 391-398.

Suga, K., and K. Abe, 2000. Nonlinear eddy viscosity modeling for turbulence and heat transfer near wall and shear-free boundaries. Int. J. Heat Fluid Flow, 21, pp. 37-48.

Thompson, B. E., O. Bouchery and K. D. Lowney, 1995. Refractive-index-matching laser velocimetry for complex nozzle entrance flow. Laser Anemometry - 1995, ASME FED, 229, pp. 365-370.

Valueva, E. P., and V. N. Popov, 1985. Numerical modeling of mixed turbulent convection of water at subcritical and supercritical pressures. Thermal Engineering, 32 (9), pp. 525-529.

Vilemas, J. V., P. S. Poskas and V. E. Kaupas, 1992. Local heat-transfer in a vertical gas-cooled tube with turbulent mixed convection and different heat fluxes. Int. J. Heat Mass Transfer, 35 (10), pp. 2421-2428.

Vukoslavcevic, P. V., and J. M. Wallace, 2002. The simultaneous measurement of velocity and temperature in heated turbulent air flow using thermal anemometry. Meas. Sci. Technol., 13, pp. 1615-1624.

Vukoslavcevic, P., and J. M. Wallace, 1996. A 12-sensor hot-wire probe to measure the velocity and vorticity vectors in turbulent flow. Meas. Sci. Technol., 7, pp. 1451-1461.

Wallin, S., and A. Johansson, 2000. An explicit algebraic Reynolds stress model for incompressible and compressible turbulent flows. J. Fluid Mech., $\underline{403}$, pp. 89-132.

Wang, X., X. Xu, and R. H. Pletcher, 2005. Large eddy simulation of supercritical $\mathrm{CO}_{2}$ pipe flow with constant wall heat flux. AIAA paper 2005-4995,17th AIAA Computational Fluid Dynamics Conference, Toronto, Ontario, June 6-9.

Weinburg, R., 1972. Experimental and theoretical study of buoyancy effects in forced convection to supercritical pressure carbon dioxide. Ph. D. diss., U. Manchester.

Williams, W., P. Hejzlar, M. J. Driscoll, W-J. Lee and P. Saha, 2003. Analysis of a convection loop for GFR postLOCA decay heat removal from a block-type core. Tech. report MIT-ANP-TR-095, M. I. T., March.

Winterton, R. H. S., 1998, Where did the Dittus and Boelter equation come from? Int. J. Heat Mass Transfer, 41, pp. 809-810.

Wood, R. D., and J. M. Smith, 1964. Heat transfer in the critical region temperature and velocity profiles in turbulent flow. AIChE J., 10 , pp. 180-195.

Worsoe-Schmidt, P. M., and G. Leppert, 1965. Heat transfer and friction for laminar flow of gas in a circular tube at high heating rate. Int. J Heat Mass Transfer, $\underline{8}$, pp. 1281-1301.

Xu, X., J.S. Lee, R. H. Pletcher, 2005. A compressible finite volume formulation for large eddy simulation of turbulent forced gas flows at low Mach number in Cartesian coordinates," Journal of Computational Physics, 203, pp. 22-48.

Xu, X., J. S. Lee, R. H. Pletcher, A. M. Shehata and D. M. McEligot, 2004. Large eddy simulation of turbulent forced gas flows in vertical pipes with high heat transfer rates. Int. J. Heat Mass Transfer, 47, pp. 4113-4123.

Yamagata, K., K. Nishikawa, S. Hasegawa, F. Fujii and S. Yoshida, 1972. Forced convection heat transfer to supercritical water flowing in tubes. Int. J. Heat Mass Transfer, 15, pp. 2575-2593. 